\title{
An Approach for building and evaluating \\ performance models for Component Based
}

\author{
Systems
}

\author{
By \\ Nikhil Barthwal, B.Tech \\ A thesis submitted to the Faculty of Graduate Studies and Research in \\ partial fulfilment of the requirements of the degree of \\ Master of Applied Science
}

Ottawa-Carleton Institute for Electrical and Computer Engineering

Faculty of Engineering

Department of Systems and Computer Engineering

Carleton University

Ottawa, Ontario, K1S 5B6

Canada

November, 2004

(C) Nikhil Barthwal, 2004 


$\begin{array}{ll}\begin{array}{l}\text { Library and } \\ \text { Archives Canada }\end{array} & \begin{array}{l}\text { Bibliothèque et } \\ \text { Archives Canada }\end{array} \\ \begin{array}{l}\text { Published Heritage } \\ \text { Branch }\end{array} & \begin{array}{l}\text { Direction du } \\ \text { Patrimoine de l'édition }\end{array} \\ \begin{array}{l}\text { 395 Wellington Street } \\ \text { Ottawa ON K1A ON4 }\end{array} & \begin{array}{l}\text { 395, rue Wellington } \\ \text { Ottawa ON K1A ON4 } \\ \text { Canada }\end{array}\end{array}$

Your file Votre référence

ISBN: 0-494-00734-6

Ourfile Notre référence

ISBN: 0-494-00734-6

NOTICE:

The author has granted a nonexclusive license allowing Library and Archives Canada to reproduce, publish, archive, preserve, conserve, communicate to the public by telecommunication or on the Internet, loan, distribute and sell theses worldwide, for commercial or noncommercial purposes, in microform, paper, electronic and/or any other formats.

The author retains copyright ownership and moral rights in this thesis. Neither the thesis nor substantial extracts from it may be printed or otherwise reproduced without the author's permission.
AVIS:

L'auteur a accordé une licence non exclusive permettant à la Bibliothèque et Archives Canada de reproduire, publier, archiver, sauvegarder, conserver, transmettre au public par télécommunication ou par l'Internet, prêter, distribuer et vendre des thèses partout dans le monde, à des fins commerciales ou autres, sur support microforme, papier, électronique et/ou autres formats.

L'auteur conserve la propriété du droit d'auteur et des droits moraux qui protège cette thèse. $\mathrm{Ni}$ la thèse ni des extraits substantiels de celle-ci ne doivent être imprimés ou autrement reproduits sans son autorisation.
In compliance with the Canadian

Privacy Act some supporting forms may have been removed from this thesis.

While these forms may be included in the document page count, their removal does not represent any loss of content from the thesis.
Conformément à la loi canadienne sur la protection de la vie privée, quelques formulaires secondaires ont été enlevés de cette thèse.

Bien que ces formulaires aient inclus dans la pagination, il n'y aura aucun contenu manquant.

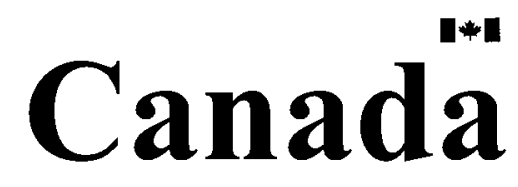




\section{Abstract}

Component based Software Engineering (CBSE) provides rapid development using well-tested components with established properties. Performance and other non-functional properties can also be analyzed by building models from sub-models, calibrated for the components. Further development of components creates libraries of parameterized components, which can provide alternatives, from which a choice can be made. The choice can be governed by functional and non-functional goals, which can be expressed partly by constraints on the assembly, and partly as non-functional goals such as performance targets. This thesis describes a systematic approach to exploring systems by capturing requirements and building new services. This involves extending a modeling framework; an XML-based language CBML (Component Based Modeling Language) defining layered queuing models, by notions of groups and services. This approach supports the goal of flexible and rapid prototyping of systems. Techniques for evaluating and screening alternative configurations are also described. 


\section{Acknowledgement}

First of all, I would like thank my supervisor, Professor Murray Woodside, for his support and knowledge. His support has been instrumental in success of this work.

I would like to thank my parents for their moral support and constant encouragement; and my brother, Tarun, whose smile always boosted my spirits.

Finally I would also like to thank all the people at Carleton University I have had the pleasure to meet. They made Carleton a great working environment where learning was fun.

Financial support from Communication and Information Technology Ontario (CITO) and AT\&T Research Labs is greatly appreciated. 


\section{Table of Contents}

$\begin{array}{ll}\text { Abstract } & 3\end{array}$

$\begin{array}{ll}\text { Acknowledgements } & 4\end{array}$

$\begin{array}{ll}\text { List of Tables } & 9\end{array}$

$\begin{array}{lr}\text { List of Figures } & 10\end{array}$

Table of Contents $\quad 5$

Chapter 1: Introduction

$\begin{array}{ll}1.1 \text { Motivation and Objectives } & 14\end{array}$

$\begin{array}{ll}1.2 \text { Thesis Goals } & 16\end{array}$

$\begin{array}{ll}\text { 1.3 Thesis Contributions } & 17\end{array}$

$\begin{array}{ll}\text { 1.4 Thesis Organization } & 18\end{array}$

Chapter 2: Thesis Background

2.1 Software Performance Engineering (SPE) 20

2.1.1 Introduction to Software Performance Engineering (SPE) 20

$\begin{array}{ll}\text { 2.1.2 Software Bottlenecks } & 22\end{array}$

2.2 Methods for building Performance Models $\quad 24$

2.2.1 Modeling with Stochastic Petri Nets 24

$\begin{array}{ll}\text { 2.2.2 Modeling with Queuing Networks } & 25\end{array}$ 
2.2.3 Modeling with Layered Queuing Networks (LQN)

2.3 eXtended Markup Language (XML) 32

2.3.1 Overview of XML 32

$\begin{array}{ll}\text { 2.3.2 XML Schema } & 34\end{array}$

2.4 Introduction Component based Software Engineering 35

2.4.1 Component Based Software Engineering (CBSE) 35

2.4.2 UML Notations for component models 37

2.4.3 Performance Modeling in CBSE 38

2.5 Component Based Markup Language (CBML) 40

2.5.1 Overview of CBML $\quad 40$

2.5.2 Structure of CBML $\quad 42$

Chapter 3: Systematic approach to building services

3.1 Assembly of Services $\quad 46$

$\begin{array}{ll}\text { 3.1.1 Description of Services } & 47\end{array}$

$\begin{array}{ll}\text { 3.1.2 Software Components } & 48\end{array}$

3.1.3 An Example of an Assembly of Service $\quad 49$

$\begin{array}{ll}3.2 \text { Performance concerns } & 50\end{array}$

3.3 Generating Models 52

3.3.1 Modeling assembly of services through templates 52

3.3.2 Modeling software component interfaces

3.3.3 Guiding composition process $\quad 54$

3.4 Evaluation and ranking of alternatives based on requirements 56 
3.4.1 Capturing requirements though constraints

3.4.1.1 Hard constraints

3.4.1.2 Soft constraints

3.4.2 Evaluating suitable alternatives

Chapter 4: Library Manager Tool

4.1 Overview of the Library Manager Tool 72

4.2 Managing components with the Library Manager Tool 74

4.2.1 Group controls

4.2.2 Component controls

4.2.2.1 Searching for Components 77

4.2.2.2 Group settings for a Component 79

4.2.2.3 Slot settings for a Component 80

4.2.2.4 Port settings for a Component 82

4.3 Building systems from components 83

4.3.1 Tree view of component assembly 84

4.3.2 Building systems manually using the tool 85

4.3.3 Automatic assembly of system 88

4.4 Conclusion 90 
Chapter 5: A Case Study: A Distributed Telephone Switch

5.1 The Distributed Telephone Switch (DTS)

5.1.1 Need for performance engineering

5.1.2 Architecture of a DTS

5.2 Modeling the Distributed Telephone Switch (DTS)

5.2.1 Template model of a telephone Switch 96

5.2.2 Constraining the system 97

5.2.3 Components of a telephone switch 99

5.3 Building and evaluating model

5.4 Interpreting the results

Chapter 6: Conclusions

6.1 Conclusions to the work

6.2 Limitations

6.3 Future Research

References

Appendix A: Diagrams for components used in example of Chapter 3

Appendix B: CBML code for components used in case study of Chapter 5 


\section{List of Tables}

2-1 Some Graphical Notations in LQN models 31

2-2 Some Graphical Notation in UML Notation 38

3-1 Hard constraints for online banking example $\quad 58$

3-2 Soft constraints for our online banking example with penalty prices in brackets \{\}$\quad 59$

3-3 Description of the six components $\quad 62$

3-4 Summary of penalty values for all alternatives at $N=50 \quad 70$

5-1 Hard constraints for the distributed telephone switch example 98

5-2 Soft constraints for Distributed Telephone Switch example with $\begin{array}{ll}\text { penalties shown in braces }\{\} & 98\end{array}$

5-3 Abbreviation used for slot of template $\quad 99$

5-4 Abbreviation used for slot of template $\quad 100$

5-5 List of all alternatives generated by the tool $\quad 108$

5-6 List of all feasible alternatives with their respective penalties 115 


\section{List of Figures}

1-1 Multiple interfaces to the same system 15

2-1a UML diagram for synchronous message $\quad 28$

2-1b UML diagram for asynchronous message $\quad 28$

2-1c UML diagram for forwarding message $\quad 29$

2-2 An LQN model for a web server $\quad 32$

2-3 An example of CBML component 41

2-4 Structure of lqn-model in UML notation $\quad 43$

2-5 Structure of lqn-submodel in UML notation $\quad 43$

2-6 Structure of slot in UML notation $\quad 44$

2-7 Structure of slot in UML notation $\quad 44$

2-8 Structure of task in UML notation $\quad 45$

3-1 A typical web service scenario with multiple types of clients and set $\begin{array}{ll}\text { of collaborating objects } & 47\end{array}$

3-2 Two alternative ways of implementing a read operation 49

3-3 Use Case diagram for Assemble of Services for our online banking $\begin{array}{lr}\text { example } & 50\end{array}$

3-4 Principle of connecting a port by matching services 54

3-5 An example of classification of components in groups 56

3-6 A plot for $y=[x]^{+} \quad 57$ 
3-7 A template model in CBMl for online banking example with three slots

3-8 Plot of the function $f(x)$ for hard constraint (1) in Table 3-1 showing feasible and infeasible areas of response times

3-9 Plot of the function $f(x)$ for hard constraint (2) in Table 3-1 showing feasible and unfeasible areas of response times

3-10 Plot of the function $f(x)$ for hard constraint (3) in Table 3-1 showing feasible and unfeasible areas of response times

3-11 Plot of the function $f(x)$ for soft constraint (1) in Table 3-2, showing penalized and unpenalized regions

3-12 Plot of the penalty for soft constraint (1) in Table 3-2

3-13 Plot of the function $f(x)$ for soft constraint (2) in Table 3-2, showing penalized and unpenalized regions

3-14 Plot of the penalty for soft constraint (2) in Table 3-2

3-15 Plot of the function $f(x)$ for soft constraint (3) in Table 3-2, showing penalized and unpenalized regions

3-16 Plot of the penalty for soft constraint (3) in Table 3-2

3-17 Plot of total penalty for all constraints shown in Table 3-2

4-1 UML relationship diagram for various elements of modeling framework

4-2 Main panel with Groups pane active

4-3 Main panel with Component pane active

4-4 Search window for components with Groups selection pane active

4-5 Search window for components with Services selection pane active

4-6 Result list for search operation 
4-7 Groups setting for components

4-8 List of slots for a component for slot settings $\quad 80$

4-9 Member group setting for component slots $\quad 81$

4-10 List of ports of interface and all slots of a component for port settings 82

$\begin{array}{lll}\text { 4-11 } & \text { Editing services for a port } & 82\end{array}$

4-12 Example of a tree where a binding decision needs to be taken 84

4-13 Build tree for a component with an empty slot selected for binding 86

4-14 Select a component for binding into a slot $\quad 87$

4-15 Build tree for a component with a filled slot selected for unbinding 87

5-1 Abstract view of current network $\quad 93$

5-2 Servers of call connection agent connected by internal network $\quad 94$

5-3 Servers of call connection agent connection agent connected by $\begin{array}{ll}\text { internal network } & 95\end{array}$

5-4 Template for the Distributed Telecom Switch 97

$\begin{array}{lll}5-5 & \text { First Gateway component - G1 } & 100\end{array}$

5-6 Second Gateway component-G2 101

5-7 Third Gateway component - G3 101

5-8 Fourth Gateway component - G4 102

$\begin{array}{lll}\text { 5-9 First Switch component - S1 } & 102\end{array}$

5-10 Second Switch component - S2 103

5-11 Third Switch component - S3 103

5-12 Fourth Switch component - S4 104

5-13 First Call Connection Agent component - C1 104 
5-14 Second Call Connection Agent component - C2

5-15 User throughput values for all the alternatives

5-16 User response time for all the alternatives

5-17 User throughput values for all the alternatives

5-18 Penalty value $\left(p_{i}\right)$ of soft constraint \#1 on alternatives

5-19 Penalty value $\left(\mathrm{q}_{\mathrm{i}}\right)$ of soft constraint \#2 on alternatives

5-20 Penalty value $\left(r_{i}\right)$ of soft constraint \#3 on alternatives

5-21 Penalty value $\left(f_{i}\right)$ of all constrains on alternatives 


\section{Chapter 1: Introduction}

\subsection{Motivation and Objectives}

Component Based Software Engineering (CBSE) is of interest in both research and practice [20], [28]. There are several reasons for it. Reusing existing components allows one to build high quality systems rapidly. Exploiting pre-existing functionality and data not only saves time and money but also reduces uncertainty.

One way to enhance quality of the product is to be able to predict performance properties of a system being built in advance. However performance is often ignored during the software development cycle. Typically the approach taken in practice is to design and implement the system and tune it for performance later on. Often this is too late to detect performance failures and might involve significant redesigning and reimplementation of the system to meet performance objectives.

The use of CBSE makes it easier to predict the performance of a system in advance thus preventing performance disasters. The components used for building systems can be calibrated in advance. A language called Component Based Markup Language (CBML) [36], [37] describes performance models of software components which can be assembled to produce a system whose performance properties are predictable. 
There may be many different configurations of components to choose from. Alternatives arise from different possible designs of a component. A major source of variability is the need to adapt to different kinds of interfaces and subsystems. Figure 1-1 shows a system with many alternative interfaces. Each interface requires its own driver, and it may also require different handling of the interface stream e.g. a cell phone interface has to handle abbreviated commands. Many systems require the capability to interface to different operating systems, database systems, or file servers. Another source of variability is alternative performance optimizations like an optimization for small storage size versus one for handling large data sets, or for masking latency in large networks.

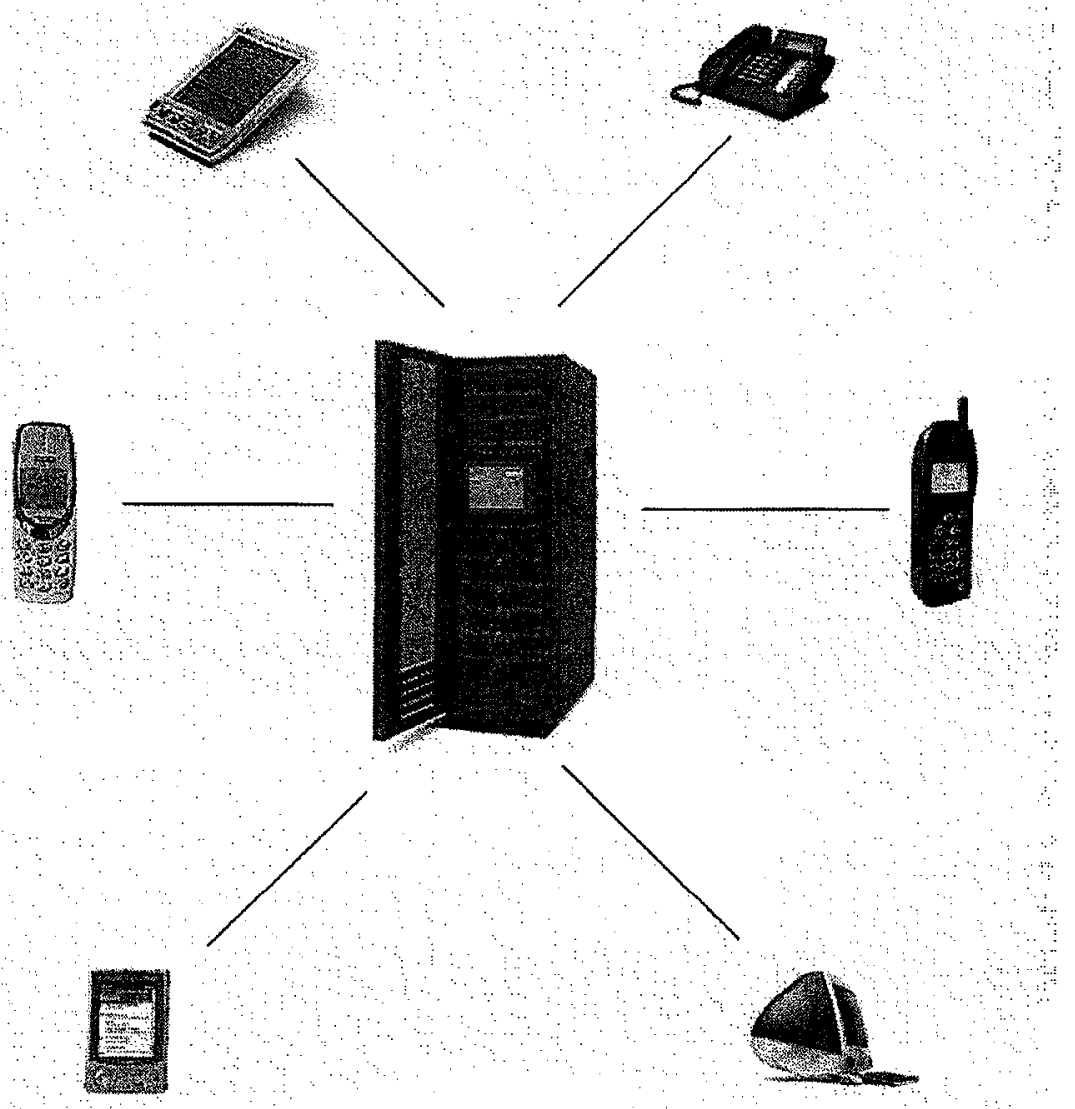

Figure 1-1: Multiple interfaces to the same system 
The goal of the work described here is to automatically assemble all possible alternative CBML models for a system which provides a set of services and screen them by user requirements to evaluate their suitability before the actual system is implemented. This enables one to select the best implementation from a performance point of view among various possible configurations.

This thesis describes an approach and a modeling environment that supports flexible rapid assembly of components to provide services with suitable performance characteristics. It could be used to create a new product configuration or to restructure an existing system to adapt to a change in workload. Performance requirements are both important and difficult to guarantee in distributed systems such as web services.

The modeling environment described here expresses constraints on component interface and functionality abstractly, by typing the ports of the components and by grouping components together by functional concerns. It is assumed that the software generation process is sufficiently parameterized so that the component chosen can be tailored to work together, and the detailed functional and interface constraints can be met.

\subsection{Thesis Goals}

The goal of this research is to develop a systematic approach for building performance model for alternative configurations of a new system and for evaluating and ranking them according to their suitability from a performance point of view. 
Performance models for components (in CBML) can be large in number and scattered in various places and hence there is a need for components to be classified in a manner that reflects all the uses of components.

Any system can be built in both top-down as well as bottom-up fashion and can be realized by different components and our approach should be flexible enough to handle this effectively.

Lastly once we have large number of alternatives, a method should exist that would evaluate and rank them according to their suitability of implementation from a performance point of view. This would require that we should be able to capture the performance requirements in a formal way which is subject to analysis. It is necessary that both strict requirements and desired features be captured and dealt in a separate yet unified manner. Further more all approaches and methods should be backed by tools that can automate some of the tasks.

\subsection{Thesis Contributions}

This thesis makes the following contributions:

- A framework for modeling system is presented, which is flexible enough to reflect multiple usages of software components in a variety of environments. The 
"component sub-model" concept for layered queuing network is extended to include classes of compatible components and interfaces to achieve this objective.

- A tool is designed, implemented, and tested to automatically create all feasible assemblies for a system based on compatible interfaces. An algorithm for automatic assembly of systems, which can enumerate all alternative realizations of a system based on satisfaction of functional and interface constraints, has been developed and it is implemented as a part of the tool.

- A new approach is defined to describe full requirements, both definite and desired, to evaluate all feasible alternatives, and to rank them using a penalty measure. Two examples presented which explains the whole process of capturing requirements and developing and evaluating models for alternatives.

\subsection{Thesis Organization}

This thesis is organized as follows. The Chapter 1 gives the motivation and objective of this research project. Chapter 2 gives some background required for this work, which includes Software Performance Engineering (SPE), various paradigms of modeling performance of systems, by various methods, Component Based Software Engineering and Component Based Markup Language. Chapter 3 explains the approach in detail including modeling the system, capturing requirements, generating alternatives, and evaluating alternatives. Chapter 4 describes the design of the tool, "Library Manager", and the algorithm for generating systems that has been developed to support the approach 
described in this thesis. Chapter 5 presents an industrial case study that applies the ideas described in this thesis to the problem of designing switches used in telecom industry. Chapter 6 gives the conclusions for this thesis and it also points out some future challenges. 


\section{Chapter 2: Thesis Background}

This chapter covers the background material for this thesis. This includes Software Performance Engineering (SPE), software bottlenecks, modeling paradigms of Queuing Network, Layered Queuing Network, and Petri Nets, Component Based Software Engineering and Component Based Markup Language (CBML). The term component model and sub-model are equivalent and are used interchangeably.

\subsection{Software Performance Engineering (SPE)}

This section provides introduction to Software Performance Engineering (SPE) and the concept of a software bottleneck.

\subsubsection{Introduction to Software Performance Engineering (SPE)}

Performance usually refers to measurable quantities like system response time as seen by the end user. Usually we have certain performance objectives for our software system. SPE is a systematic approach that provides quantitative assessment for software systems so that their performance objectives can be achieved [27]. It addresses performance issues like bottlenecks, system delays, system capacities as well as system scalability in the entire software development life cycle. 
Performance is an important issue because performance failures might delay time to market of product and may result in losing the customer. Moreover it might result in significant losses of investment. There are different approaches towards software performance engineering. We discuss each of them briefly here:

- Performance Measurement: This approach is also known as "fix-it-later" approach [19]. It is based on experimenting with an operational system and getting system performance metrics based on these experiments. Although it provides more accurate results, and the approach may able to identify performance problems such as bottlenecks, it has its own drawbacks. It can be time-consuming to develop a prototype and collect data from experiments. Also if the system has to meet a tight deadline, it may not be able to give the feedback to system designers in time. Moreover since performance problems are detected at later stage i.e. after the system is operational, they can be hard to fix.

- Performance Tuning: This is another kind of "fix-it-later" approach. In this approach, the performance improvements are done on an existing system usually by an expert who is knowledgeable of the system architecture and design. However, the improvements done at this stage are limited and it is not possible to handle the performance problems originating from design itself.

- Early prediction through performance models: The work described in this thesis is based on this approach. In the above two approaches, performance problems are often 
detected too late which makes them very hard to fix since it may involve significant design change and subsequent intensive coding as defects often exist in the architecture itself which means performance problems are introduced at an early stage. Hence, early performance prediction is very important, especially for performance-critical software systems, as it provides cost-effective ways to avoid performance failures. Performance problems if detected early can be fixed easily. The approach involves building performance model of a system before it is implemented and analyze it for performance metrics. This makes it possible to determine whether the performance objectives can be met for the emerging software system or not. This can be used as feedback to software architects or designers so that the necessary changes can be made well is advance. Further models can help us determine which configurations are suitable for implementation and which are not among many alternatives.

However, It should be stressed that performance problem is not completely avoidable irrespective of the method used.

\subsubsection{Bottlenecks}

A bottleneck is a single point of contention that limits the overall system performance [32]. Bottlenecks can be of two types:

- Hardware Bottleneck: In performance analysis, hardware bottlenecks are well understood in the conventional models of computer systems (e.g. [16], [32]). They 
usually occur at a CPU or a disk or other devices and can usually be fixed by hardware expansion.

- Software Bottleneck: In systems where there are multiple levels of client-servers, performance can also be constrained by software tasks, especially those that act as intermediate servers or "active servers" as they are called in [16]. The software bottleneck is the task that is fully utilized, while the resources it uses are underutilized, and thus it constrains the total system performance [19].

In Software bottlenecking, when a software task is highly utilized, it will "push back" on its clients which makes them appear to be saturated too. In systems that are deeply layered, software blocking spreads upwards and may affect a large part of the system. At the same time, the resources that are used by these tasks are underutilized. A detailed discussion of software bottlenecking can be found in [19].

A typical example of software bottlenecking is the case when a single-threaded task is waiting for an $\mathrm{I} / \mathrm{O}$ operation. Another example is shown in [19] where the model has two levels of servers. The active server, which has the roles of both client to its lower layer and server to its upper layer, could become the software bottleneck in the system if it is not multithreaded.

The solution for eliminating software bottlenecks is by multithreading or cloning the software tasks that causes performance constraints [19]. By doing so, the utilization of 
the underlying resources can be enhanced and the overall system performance can be improved.

\subsection{Methods for building Performance models}

This section provides overview of three main methods for building software performance models. They are Stochastic Petri Nets, Queuing Network models, and Layered Queuing Network. The following sections will describe them briefly.

\subsubsection{Modeling with Stochastic Petri Nets}

Petri nets were introduced as a model of computation for concurrent systems by Carl Adam Petri in 1962. A detailed introduction can be found in the book [1]. A Stochastic Petri Net model is a high-level model which generates a stochastic process. The stochastic process is then analyzed via Markov Chain technique. The performance measures are then obtained from the steady state probabilities. The main components in a Petri net are places and transitions. Places represent states or resources in the system while transitions model the activities. Places are graphically represented as circles and transitions are graphically represented as bars. Places and transitions are connected via a set of directed arcs. Places may contain tokens which are graphically represented as dots inside the circle. Tokens move through the network (from place to place) according to certain rules. In Stochastic Petri Nets, a transition is enabled if its input places have one or more tokens. Each transition has a firing time which is an exponentially distributed random event. An enabled transition has a probability of firing but it can only fire after a period of time, which is exponentially distributed, has elapsed. In addition, only one 
enabled transition can be fired at any time. When a transition fires, a token is removed from the input places and added to the output places. The current state of the model is determined by the number of tokens in each place and this is also called a marking. These markings (states) generate the underlying Markov Chain models. The performance evaluation is achieved by solving these Markov Chain models. Lopez-Grao et al. [15] uses stochastic Petri nets for software performance analysis.

Although the Stochastic Petri Net models are useful for performance modeling of computer systems that exhibit concurrency, synchronization and randomness, they suffer from state space explosions which make performance computation very hard.

\subsubsection{Modeling with Queuing Networks}

Queuing Network models consist of a number of servers and customers (which are also known as tokens or jobs). Customers make requests to the servers. Depending on the status of the server, the requests may be queued if the server is busy or get processed if the server is idle. There is a special kind of server called delay server or infinite server where customers get serviced immediately without waiting in a queue. Queuing Network models can be classified into three types:

- Open queuing network: In this type of queuing network, customers can enter or leave the network. There is no fixed population of customers. 
- Closed queuing network: In this type of queuing network, customers circulate the servers; therefore, the population is fixed. No customer is allowed to enter or leave the network.

- Mixed queuing network: In mixed queuing network, some customers may leave or enter the network, whereas some circulate in the network. Hence it's a mixture of the two above kinds of queuing network.

In a queuing network model, customers are grouped based on their statistical behavior. As a result, there may be multiple classes of customers in one network. The main parameters for a queuing network model are mean service time per request per server and the visit ratio that a customer makes to the server. Other factors like scheduling policy and the queue size of the server may also affect the behavior of a queuing network. The queuing network models can be solved analytically or simulated to obtain throughput and mean response time.

However, Queuing networks have a drawback. They are unable to capture contention for software resources. They model the user requests as pure customers and the devices as pure servers and hence cannot model an intermediate software server that accepts and sends requests from and to other software processes. Hence they also cannot incorporate the case where a job has phases of execution with an early reply. Further parallelism that involves forks and joins is hard to model in Queuing Network. Daniel A. Menasce in his paper [18] discusses a technique to overcome this problem by a two-level iterative 
queuing modeling of software contention where there would be two queuing networks one for software resources and the other one for hardware resources. This is an elementary kind of Layered Queuing Analysis.

\subsubsection{Modeling with Layered Queuing Networks (LQN)}

The Layered Queuing Network (LQN) models, which were previously called Stochastic Rendez-Vous Networks (SRVN) models, extend the traditional queuing network models by allowing for an arbitrary number of software layers which may act as both clients or servers at different layers [22], [33]. Thus LQN models [19] can model both software and hardware contention, and the impact of layers on service time. They form the basis for component based models and CBML introduced in Section 2.5. LQN models are suitable for many important classes of systems, including those using Remote Procedure Calls (RPC) and distributed systems such as distributed database system, telecommunication systems and agent systems [14], [23], [34].

The structure of an LQN model resembles the software architecture of the system. It is expressed as a set of objects called "tasks" offering services (like methods) called "entries"; entries of one task make requests to entries of others at lower layers. They also have something called "activities" which are the smallest unit of computation. They can model sequential and parallel executions by And Forks and Joins, alternative executions by Or Forks and Joins as well as repetitive executions. They are a part of task and they can send requests to entries. A task that does not receive any requests but only sends requests to lower layers is called a "reference task". A reference task is the load generator 
of the system. Tasks are executed on processors which represent physical resources such as CPUs and disks. A Task through it entries makes calls to entries of lower tasks. There are three kinds of calls:

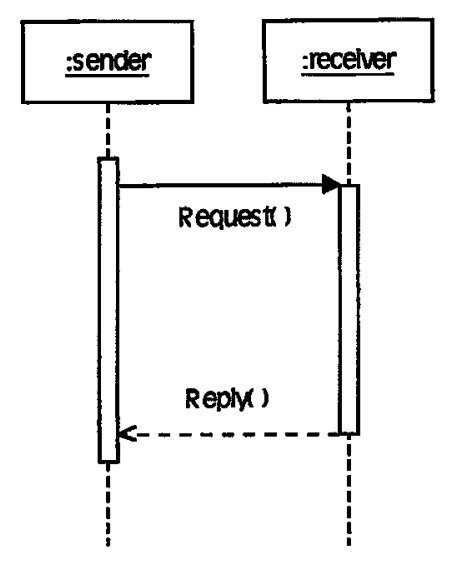

Figure 2-1a: UML diagram for synchronous message

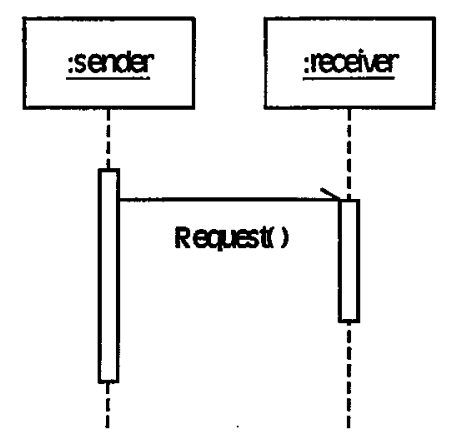

Figure 2-1b: UML diagram for asynchronous message

- Synchronous message: In this case, sender sends the message and is blocked, waiting for the reply. The UML diagram for this type of interaction is shown below in Figure 2.1a: 
- Asynchronous messages: Sender sends the message and then continues doing its work and there is no blocking in this case. The UML diagram for this type of interaction is shown below in Figure 2.1b:

- Forwarding messages: The first sender sends a synchronous message. But the receiver does not reply directly, it does partial processing and then forwards the messages to a third object. The third object will reply back to the original sender. There may be multiple intermediate receivers that forward the request further. The last receiver is expected to send the reply back to the original sender. For example, a typical forwarding interaction is shown in Figure 2.1c where receiverA receives a message from sender and forwards it to receiverB. The return of this message is sent by receiver $B$ and is directly received by sender.

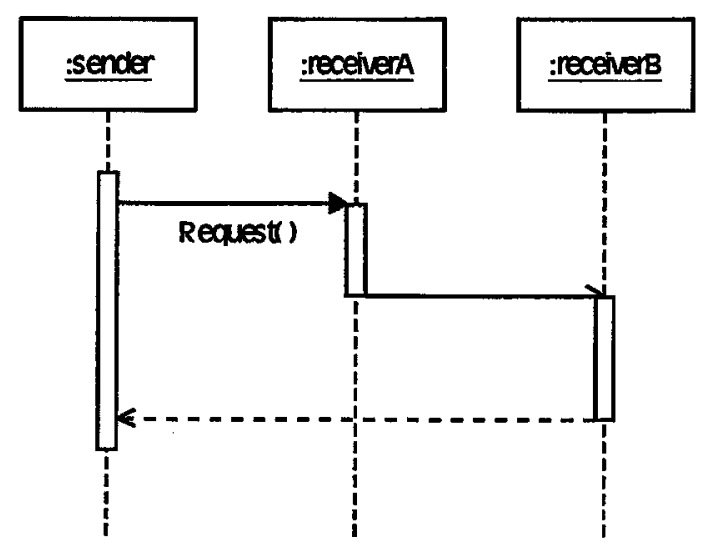

Figure 2-1c: UML diagram for forwarding message 
Also, we can have more than one phase of execution in a synchronous call (including the intermediate receivers in case of forwarding). The first phase consists of the activities that occur between a task accepting the synchronous request and replying (or forwarding) it back. The second phase consists of the activities that occur after the reply. With the introduction of second phase, the sender can be released from its blocking state earlier which can promote some concurrency between the sender and the receiver. This was also termed as an "aggressive" reply in [9].

The performance measures obtained by solving the LQN models are detailed at different levels which include service times of each entry, throughput and utilization per phase of each entry and each task, the utilization and waiting time of each processor and some values of variance and bounds. For this thesis work, the metrics of interests are response times, throughput and utilization of each task and each processor.

An example of LQN model is shown below in Figure 2-2. This is a model for a typical Web Server. In this example, clients send requests to the web server. The listener for the web services accepts these requests and based on the types of the requests and they are forwarded to the corresponding services in the server pool. These services need to access the disks. After the results have been worked out, they are sent back to the clients. The parameters such as CPU demands and number of interactions between entries are not shown in this model. Some commonly used LQN notations are shown in Table 21. 


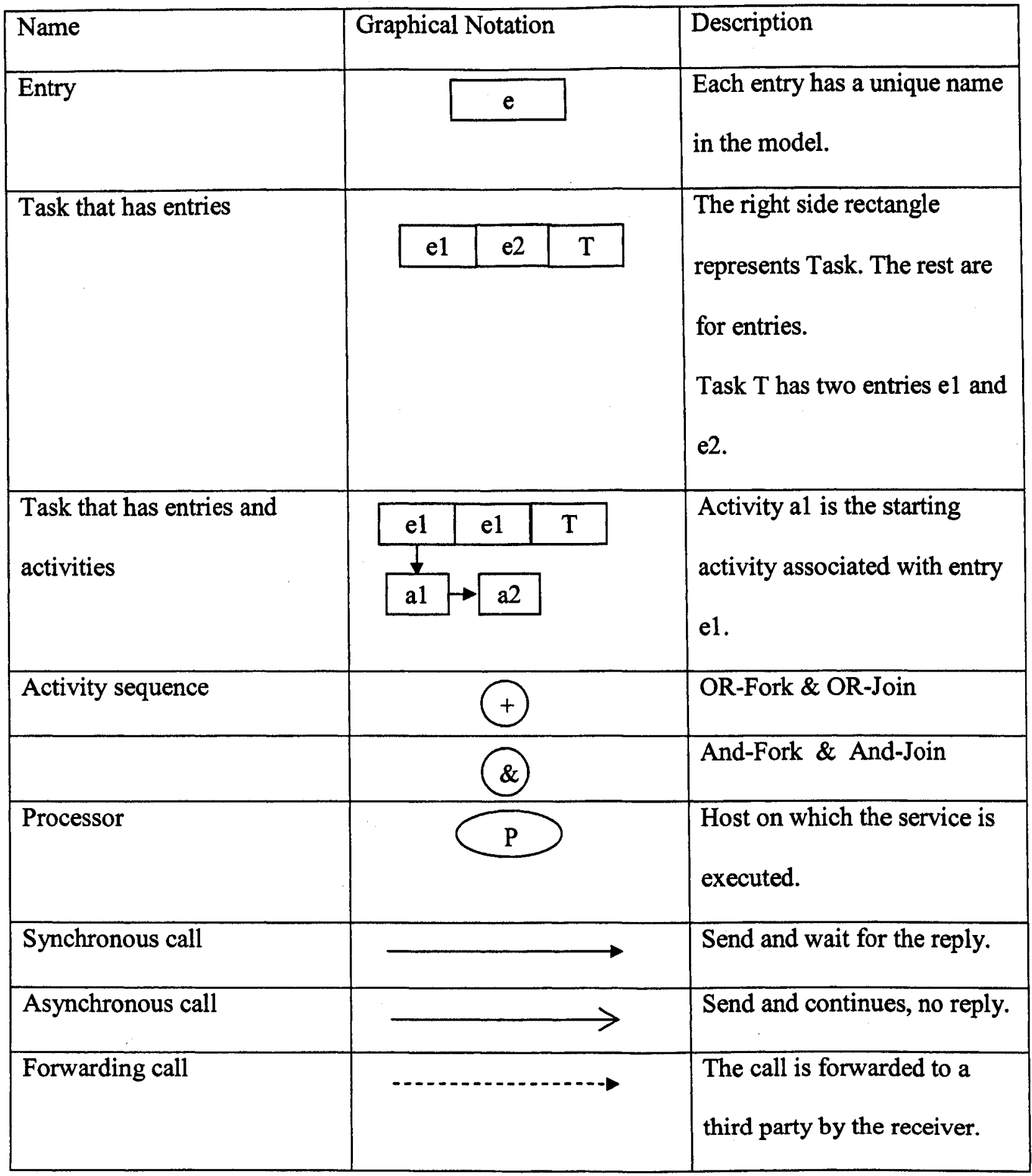

Table 2-1: Some Graphical Notations in LQN models 


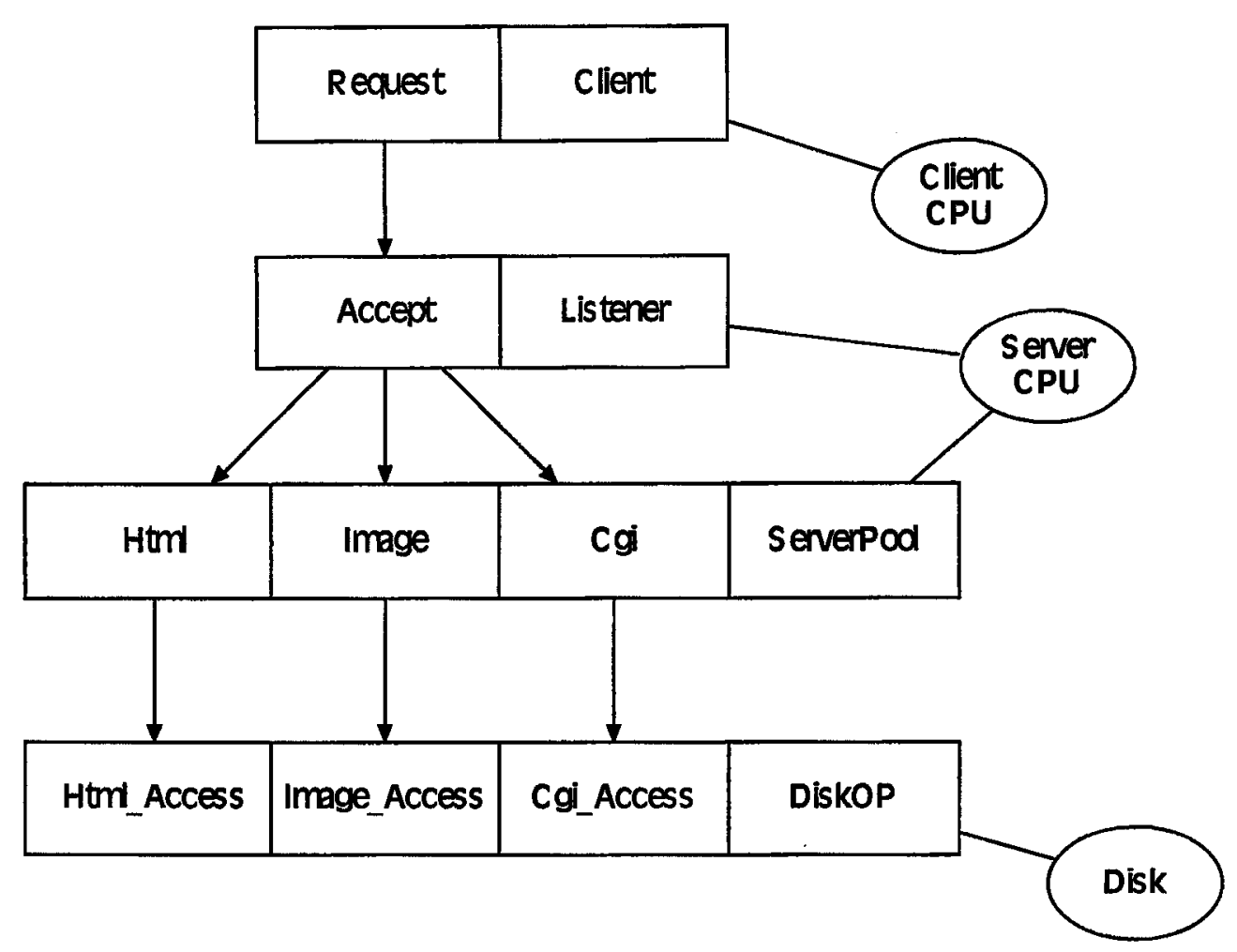

Figure 2-2: An LQN model for a web server

\section{3 eXtended Markup Language (XML)}

This section gives some background of XML language and XML Schema.

\subsubsection{Overview of XML}

XML is derived from Standard Generalized Markup Language (SGML) and it used to describe structured data. It has been introduced by the World Wide Web Consortium (W3C) and is a text-format based platform-independent markup language. 
XML has some resemblance to HTML, i.e. its human readable and editable. However, it defines its own tags (unlike HTML which has a fixed set of tags). This turns out to be a big advantage because it results in separation of syntax and semantics. The content of an XML document is independent from its rendering. Because of this, XML is now playing an increasingly important role in data exchange over the web.

An XML document is mainly composed of nested elements with some or no attributes which are enclosed by start tags and end tags. Attributes carry some assigned values. Elements are nested and they can have attributes that are assigned values in the start tags. The whole document is contained by a distinguished root element which appears at the very beginning of the document. An example of a simple XML file is shown below:

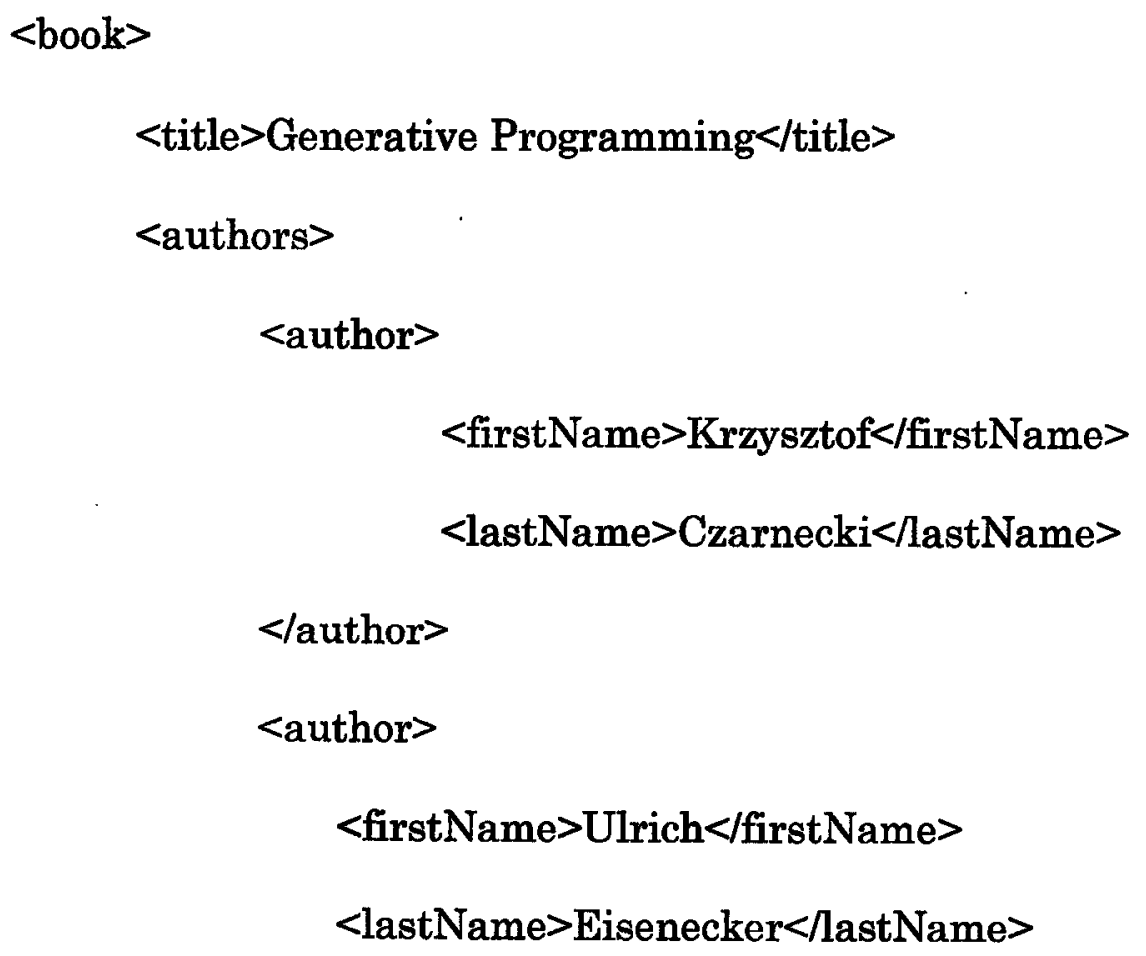




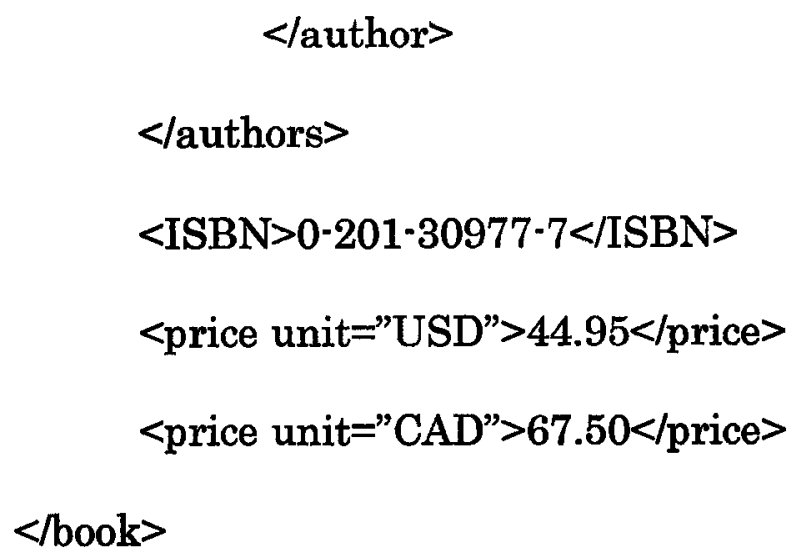

The root element in this example is book. The element price has an attribute named unit which has the values of "USD" and "CAD".

An XML document is said to be well-formed if its logical structure obeys certain formatting rules which enables a standard XML parser to parse it. For instance, a start tag must have a matched end tag. Tags must be strictly nested if the element has subelements. An XML document is said to be valid if it conforms to a pre-defined DTD (Document Type Definition) or to an XML schema which specifies the rules governing the structure of an XML document. In this thesis work, the validation of an XML document is performed against the XML schema which will be introduced in the next section.

\subsubsection{XML Schema}

The W3C Schema is a W3C Recommendation which intends to describe and constrain the content of a set of similar XML documents. It constrains the allowed structure of the XML documents with precision. It also imposes constraints on the datatypes that are 
permitted at individual locations within that structure [38]. The XML Schema Definition (XSD) language is also in the form of an XML language. It is much more powerful than the previous DTD (Document Type Definition) in terms of datatyping and constraining the document content. In an XML document, the content of the elements can be nested. The XML schema specifies content models for element types which describe the frequencies and the orders in which elements can appear in the content of the element type. It can also specify the possible attributes and their data ranges for an element type. Elements that have neither child elements nor possess attributes are said to be of simple type, whereas elements having child elements or attributes are said to be of complex type. Some of the elements have attributes and attributes always have simple types.

The relationship between an XML schema and its valid XML documents is similar to that of a class and its instances in the Object-Oriented programming language. Therefore, an XML schema can be used to validate a set of XML documents.

\subsection{Component based Software Engineering and Markup language}

This section gives some background of CBSE. It also reviews some research work of performance engineering in this area.

\subsubsection{Component Based Software Engineering (CBSE)}

Component Based Software Engineering (CBSE) has emerged as a promising paradigm in both academia and industry. CBSE emphasizes on building system by reusing high quality configurable software components. This reduces its development cost as well as 
time-to-market and ensures higher reliability, better maintainability, and better quality by exploiting reusability. In the traditional approach, when a software system is going to be developed, the implementation has to be done from scratch.

The development process of CBSE is quite different from the traditional software engineering approach. It can be generalized as having the following five steps:

1) Requirement analysis

2) System architecture

3) Component selection

4) System integration

5) System testing

There are two central parts in CBSE - components and architecture. Components are the basic building blocks for a system. Architecture describes how components are assembled into an application system. There is no unified definition for software components. Szyperski [28] and Heineman \& Councill [20] gave their own definitions of software components. In general, the following characteristics are included in a software component:

- It is an independent, compositional and deployable unit.

- It has clearly defined and documented interfaces interacting with other components.

- It has certain functionalities. 
- It may have explicit context dependencies such as operating system or other software components, etc.

For large and complex application, some components need to be developed separately specifically tailored to the need of the application. Components must be adaptable to the integrated system [2]. These requirements lead to architecture standards definitions and development of component technologies. Some common ones that are used today are:

- Common Object Request Broker Architecture (CORBA)

- Java Beans and Enterprise Java Beans (EJB)

- Component Object Model (COM) and Distributed COM

A comparison of these technologies can be found in [12]. The component based approach is now widely used in Software Product Line [6].

\subsubsection{UML Notations for component models}

A standardized notation for components is given by the UML 2 proposal [21]. Table 2-2 shows the UML2 notation for interfaces with ports:

\begin{tabular}{|c|l|}
\hline Graphical Notation & \multicolumn{1}{c|}{ Description } \\
\hline $\begin{array}{c}\text { <<component } \\
\text { AppServer }\end{array}$ & UML 2 notation for a component \\
& AppServer. Optionally it may \\
have two protruding rectangles in \\
\hline
\end{tabular}




\begin{tabular}{|l|l|}
\hline & $\begin{array}{l}\text { the upper right-hand corner, as in } \\
\text { UML 1. }\end{array}$ \\
\hline & $\begin{array}{l}\text { A required (output) interface of a } \\
\text { component in UML 2, that is } \\
\text { associated with a port }\end{array}$ \\
\hline 年 & component in UML 2, that is \\
& associated with a port \\
\hline
\end{tabular}

Table 2-2: Some Graphical Notations in UML Notation

A component can be replaced by any unit that has equivalent functionally and a compatible interface. Each interface has defined a formal contract of services that the component provides or requires, which in this work is described by its service type. We use the term port for an interface. There are two kinds - input ports and output ports. An input port provides a set of services whereas an output port requires a set of services.

\subsubsection{Performance Modeling in CBSE}

Research in CBSE has mainly focused on functional aspects and very little has been reported on non-functional aspects like performance. Software systems execute in different environments and this makes it hard to know the performance properties of its 
component in advance. Each different component technology has a different infrastructure and implementation and exhibits different performance characteristics which make performance predictions harder. Classical techniques and notations for performance analysis do not work. This makes performance prediction a challenging problem.

There are some existing tools (like C. U. Smith's SPE.ED [27]) but they are of limited use. Sitaraman et. al. [25] argues that software components should have performance specifications as well as functional specifications. Performance specification is considered to be a basic problem that needs attention before a system can be assembled from them. This will ensure that emerging software system will meet its performance goals. Performance specifications are necessary so that clients can decide which components will meet their goal and performance specifications should include execution time and memory capacity requirements.

Chen et. al. [4], the authors proposed an empirical approach to predict the performance of the assembled system by benchmarking and profiling. A model is then built based on the observations and this model is used as a performance predictor for a class of applications which are based on this specific component technology. Although the authors applied this approach to predict the performance of the StockOnline which is a test application and demonstrated the success, it is still time-consuming to make the observations from benchmarking and profiling. 


\subsection{Component Based Markup Language (CBML)}

Component based Markup Language (CBML) comes from applying the idea of pluggable components in LQN modeling [36].

\subsubsection{Overview of CBML}

CBML is based on XML language because XML is useful for expressing structured data like CBML. The objectives of CBML designs are [36]:

- Making it more flexible for instantiating multiple instances of a single component class.

- Making it more flexible for tailoring the component with parameters.

CBML introduces slots in a component. These slots are places where other components can be plugged in. The components that are plugged might in turn have there own slots where other components can fit in and thus might be nested. The slots are there to support the pluggable components idea of CBSE. An example of CBML component (actually a sub-model) taken from [36] is shown below in Figure 2-3.

The CBML component model boxes the various task with their entries with an interface to the box. The interface can be classified as incoming interfaces shown here by a circle and outgoing interfaces shown by a square. It supports two kinds of hosts - First kinds are the normal processors like those supported by LQN modeling which are shown inside the box (e.g. PX). The second kinds are the replaceable processors shown 
separately outside the box (e.g. P1 and P2). A replaceable processor is a kind of service serving a request for hosting tasks. Here the interfaces are separate elements other than those defined in the component body.

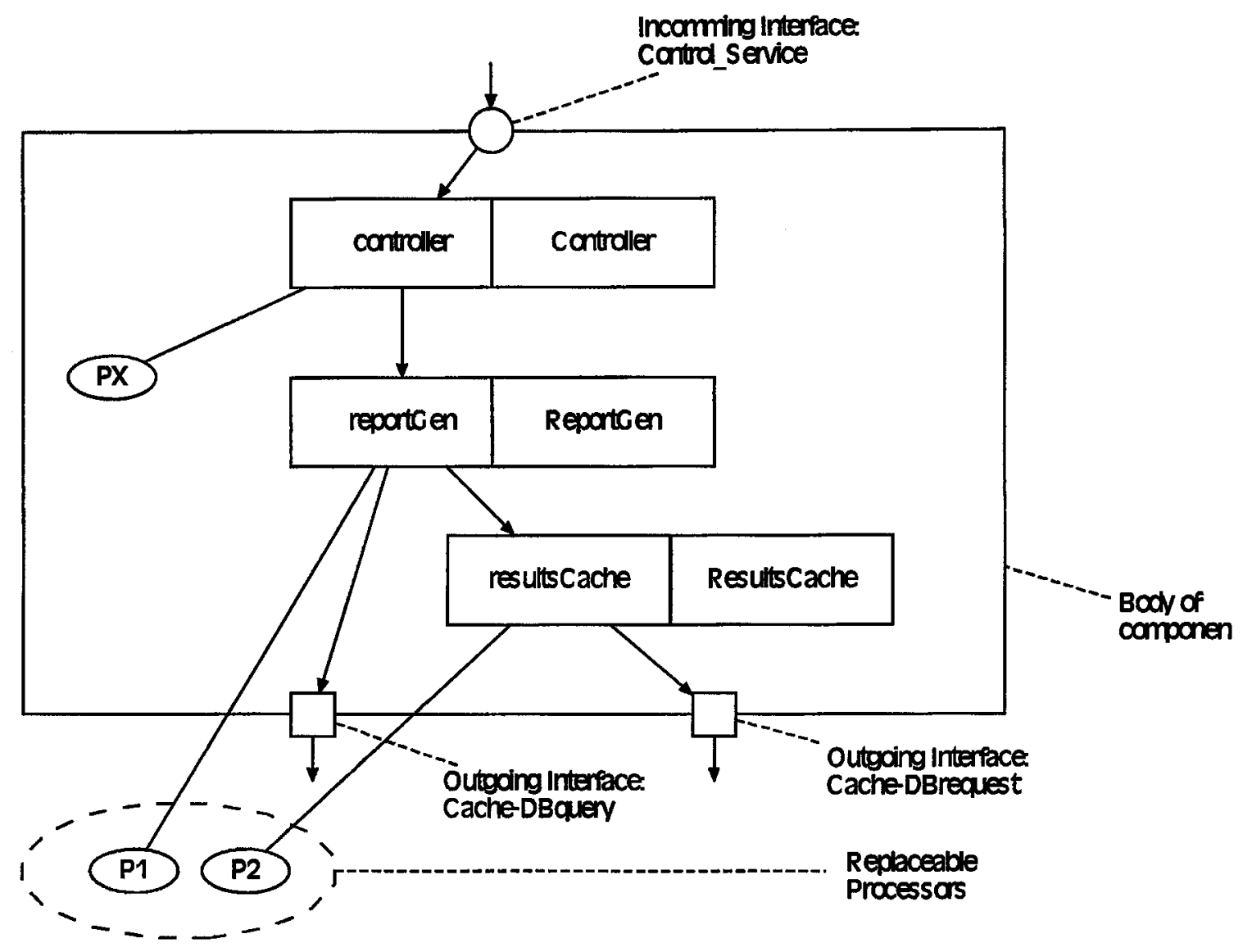

Figure 2-3: An example of CBML component

When a particular component (a sub-model - referred to as child component) needs to be fitted in slot of another component (model or another sub-model), referred to as parent component. For a particular component to fit in a slot, the interfaces of the component have to match with the interfaces of the slot. For this to happen, each incoming interface 
of the child component needs to match with each incoming interface of slot of parent component and similarly each outgoing interface of the child component need to match with each outgoing interface of slot of parent component. The incoming/outgoing ports of a component or slots are connected to the service entries in the particular component. When binding takes place, these interfaces are replaced by actual calls made from tasks of parent component to entries in tasks of child component.

\subsubsection{Structure of CBML}

The structure of CBML can be seen as classes which are collection of elements. This section describes the main elements using UML class diagram. Examples of component definition using CBML is given in appendix $B$.

Figure 2-4 and Figure 2-5 explains the basic structure of lqn model and lqn submodel. A model contains various runtime parameters like controlling run, plotting options, solver parameters, and the core model. A sub-model would contain an interface which defines the incoming and outgoing interfaces, parameters, and the core model. The structure of core is shown in the following in Figure 2-6. Figure 2-7 expands the various elements of slot and Figure 2-8 expands the various elements in task. Detailed explanation of these elements can be found in [36]. 


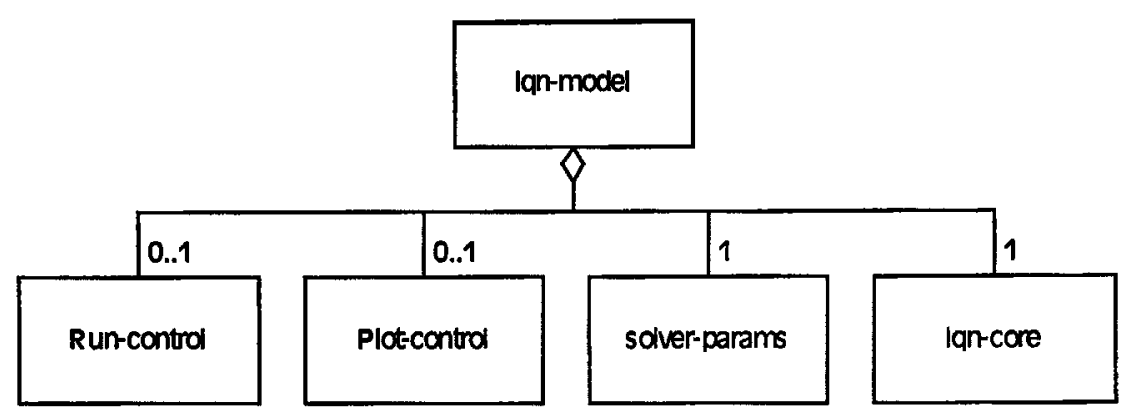

Figure 2-4: Structure of Iqn model in UML notation

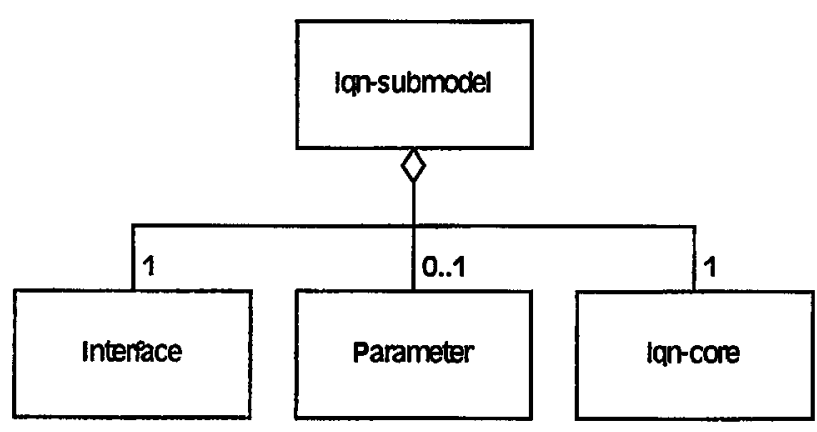

Figure 2-5: Structure of lqn sub-model in UML notation 


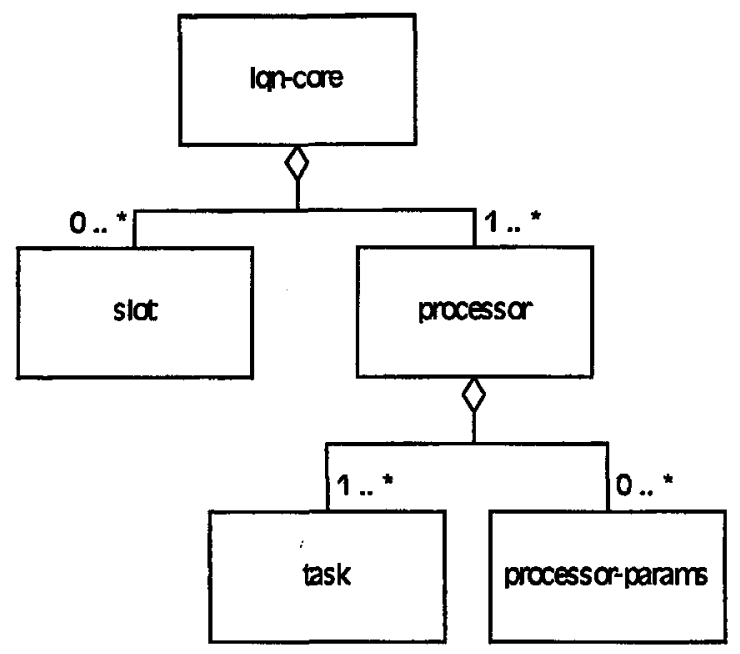

Figure 2-6: Structure of Iqn-core in UML notation

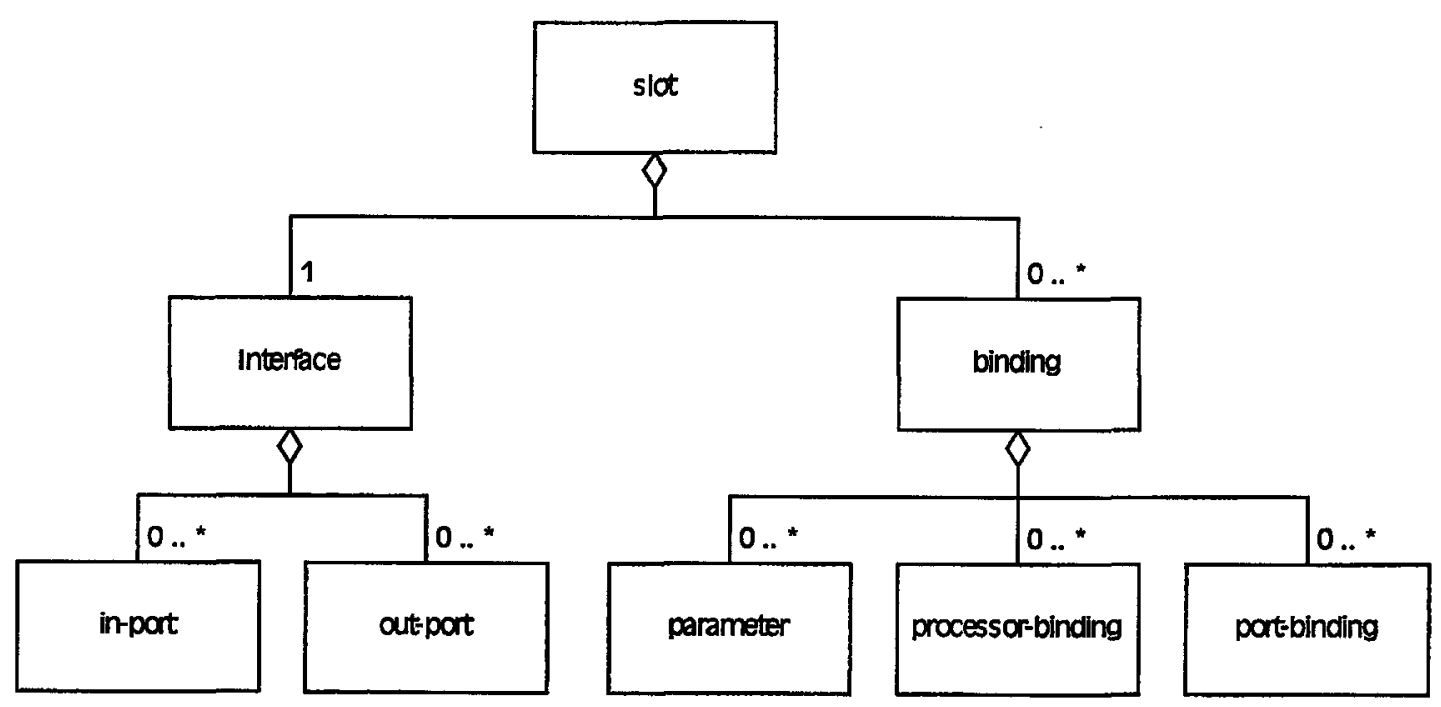

Figure 2-7: Structure of slot in UML notation 


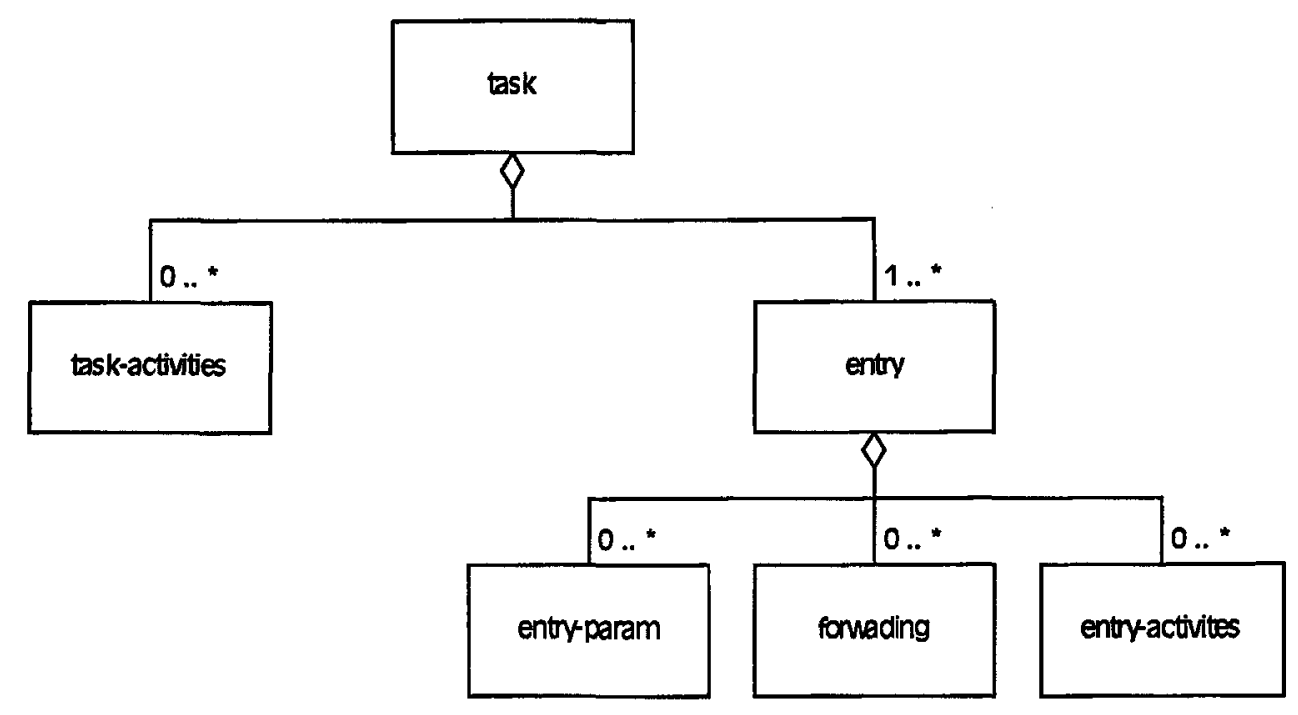

Figure 2-8: Structure of task in UML notation 


\section{Chapter 3: Systematic Approach to Building Services}

This chapter starts with a brief discussion of assembly of services and performance issues concerning them. It then explains how we can create performance models for various kinds of assembly of services from already stored models of software component (in CBML) though the use of system templates \& services description. Finally, it describes a method to evaluate them against performance goals though the use of constraints. The entire process is illustrated by an example.

\subsection{Assembly of Services}

Assembly of services is a common term used for set of software components providing a set of services by collaborating with each other using specific protocol over multiple hosts.

Assembly of services can be used in variety of applications and they are important in several ways [5]. They can free up money within an organization, by driving down integration costs, reducing expensive functionality duplication, and providing new revenue streams from existing functionality or data. Assembly of services can have a beneficial impact on strategy. They provide smaller organizations with an affordable means to access functionality or data in a form that is more readily usable by their 
internal systems. This also helps in providing functionality from a central location that would allow work to become less location dependent.

\subsubsection{Description of Services}

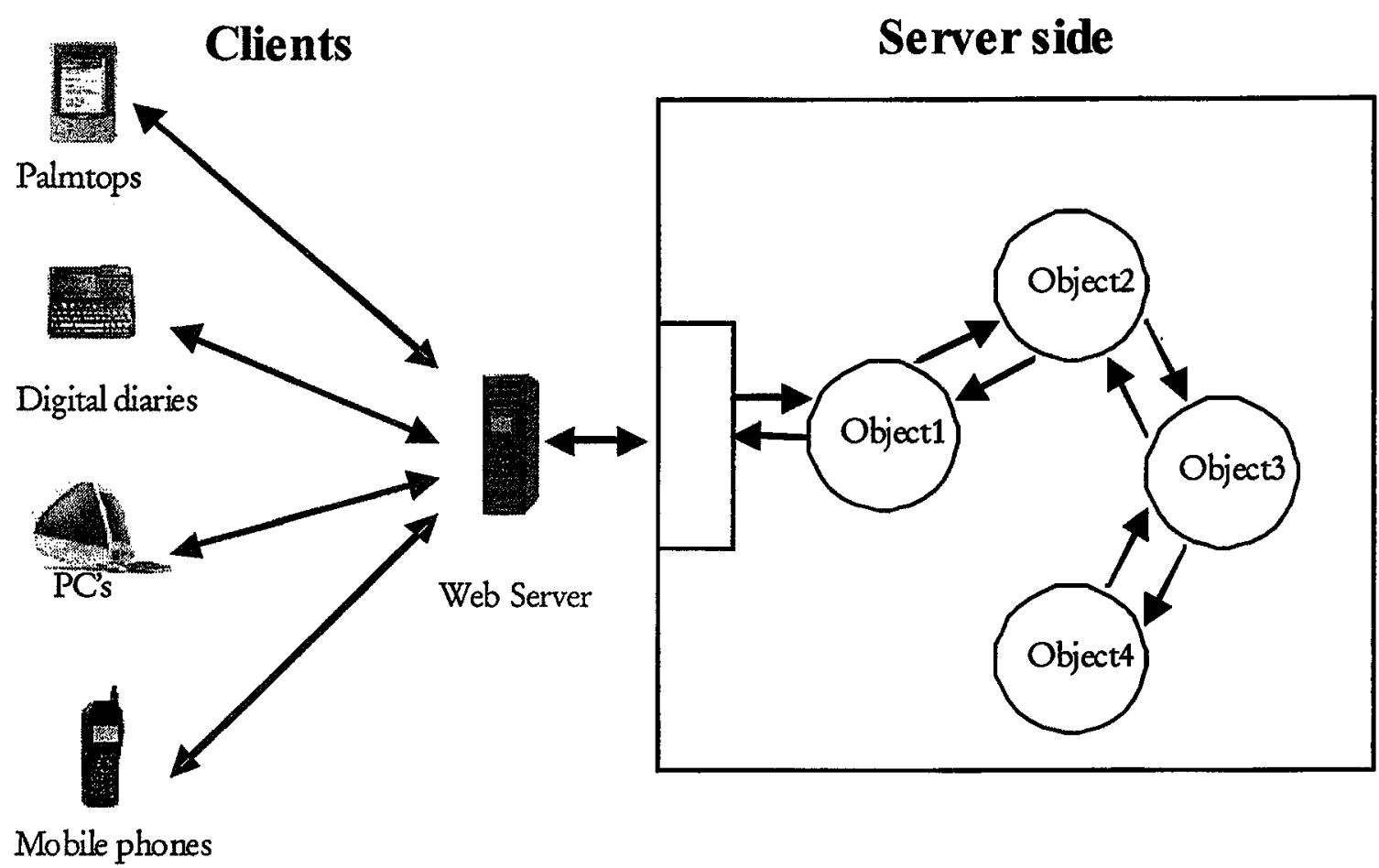

Figure 3-1: A typical web service scenario with multiple types of clients and set of collaborating objects.

A service is an operation that fulfills a certain type of request generated by a client to a server. This request is passed on to components, in form of object instances residing on the server. These objects take in the requests and return output back to client. The objects 
call each other and thus communication takes place through message passing. The functionality is usually divided hierarchically among objects.

When assembling services, there can be many different alternatives to choose from which arises from conditions like multiple implementation of same component or different ways of arranging components.

A very typical assembly of services in form of web services is shown in Figure 3-1. Multiple types of clients are shown in the figure accessing services though a web-server. These clients make a service request to the server which passes this request to particular object residing on the server which in turn might call other objects. Each client requires different kind of adaptation of the same service which can lead to alternatives.

\subsubsection{Software Components}

Software components can be implemented in more that one way. For example, data for a read operation can be cached for faster access, as illustrated in Figure 3-2. The improvement in the response time has a cost however and that is requiring more resources to do the operation. 

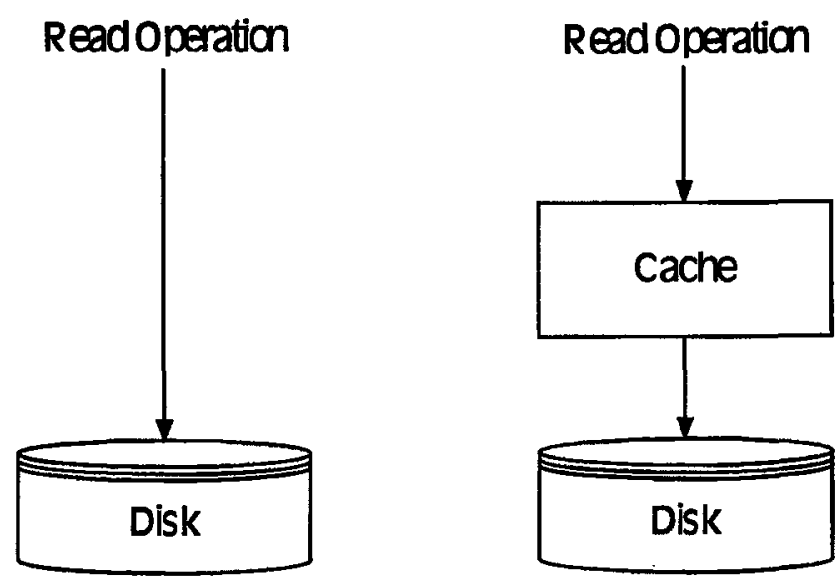

\section{Figure 3-2: Two alternate ways of implementing a read operation}

Two software components can have identical interfaces and provide/require identical services and yet have different implementations and different performance impacts. Thus various kinds of software components can be used to implement such system in a variety of ways and we would like to choose the best implementation taking various aspects into considerations.

\subsubsection{An Example of an Assembly of Service}

We would use a simple web banking application as our example. An online bank account can be accessed through various kinds of interfaces like mobile phones, PC's etc. similar to what was shown in Use Case diagram of Figure 3-1. An assembly of three services "Check account balance", "Deposit cash" and "Withdraw cash" is shown in Figure 3-3 in form of a component interaction diagram. The forward calls are shown as arrows and return calls are implicit and not shown in the figure above. 


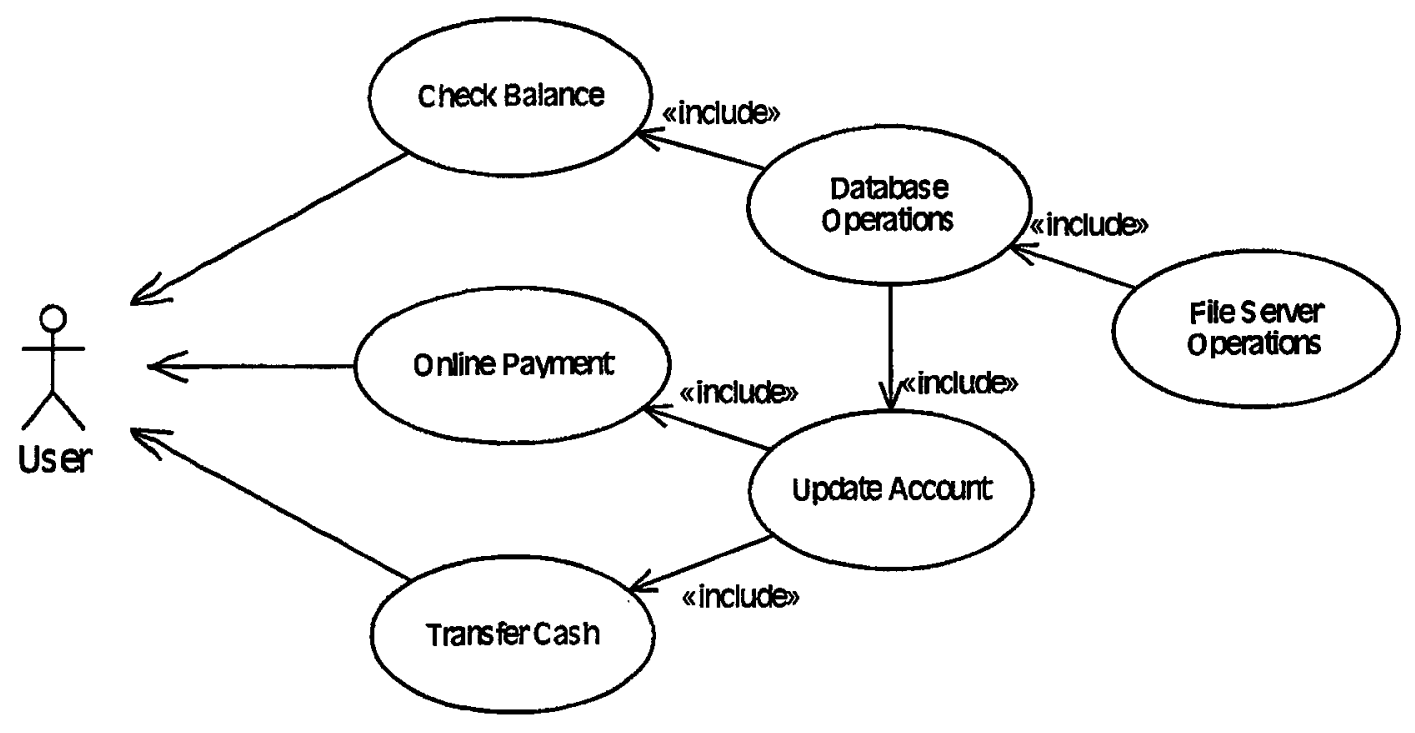

Figure 3-3: Use Case diagram for Assembly of services for our online banking example

The services that are provided are: to check the balance in an account (retrieve data from the database), to pay bills such as utilities (check the balance and update both the accounts) and to transfer cash to a credit card (update both the accounts). Software components for database operations, account updates, and file server operations are also shown in Figure 3-3.

\subsection{Performance Concerns}

Performance requirements are both important and difficult to guarantee in a distributed system like assembly of services. Performance requirements directly impact the profit of the organizations providing the services. Typically the clients of the services would not like to wait for long for their requests. Slow response time will turn away customers. The throughput of the system is directly proportional to the profitability of the organization. 
Higher the throughput of the system is, the more number of customers can use the system simultaneously and the more revenue is generated for the organization.

The most common performance measures are response time, throughput capacity and utilization. Response time is the delay to obtain a response to a request, which may be the time to the first response, or to the completion of the operation; it may be measured by a mean value, a maximum value, or a percentile (which is a target that may be exceeded by no more than a given percentage of responses). Throughput capacity describes the system's productivity in terms of requests per sec. or the number of users it can support. Utilization of a resource measures the fraction of time it is busy, and identifies actual or potential performance bottlenecks [32] and resources which may limit scalability. Each of these measures can be transformed into an operating value or a risk to the organization.

Performance requirements are captured in form of constraints on performance measures. The alternative configurations are evaluated for their proximity to the constraints. Some constraints must be met and their failure will eliminate a configuration from consideration. Some others express desirable goals and failure is penalized suitably which leads to ranking of the alternatives. Typical performance requirements might be:

- The check balance operation must have a fast response, say, less than $100 \mathrm{~ms}$ on the average.

- The other two operations should meet the same requirement if possible, but may exceed it with a penalty for a larger value, 
- A larger mean delay for online payment may be compensated by a smaller one for transferring cash.

In further discussions we will use the notation $R_{X}$ to represent the mean response time of some transaction $X, F_{X}$ to represent the mean throughput capacity of the transaction, $U_{Y}$ to represent the utilization of some resource $Y$, and $F_{Y}$ for its throughput capacity.

\subsection{Generating Models}

Generating models involves enumerating all possible alternatives based on functional requirements and building CBML models for each of these alternatives. A modeling framework is developed to achieve this goal. The main challenge here is prevent explosion of number of alternatives which is done by number of mechanisms that control and guide the composition process.

\subsubsection{Modeling Assembly of Services through Templates}

The complete system is represented by a top-level model called a template. It is a layered model in which tasks may be combined with slots, where other components can plug in, and have ports which will provide (input ports) or require the services (output ports). A component can fit in a slot if it has compatible interface i.e. all it's port match with the ports of slots. The concept of matching of ports is explained later in Section 3.3.2. 
More than one component may satisfy a slot. In our banking example we could allow for caching, or for different kind of database servers (different providers, or different models from the same provider) or file servers, providing interchangeable functionalities at the level of the system specification. Each of these components has a different implementation and hence, in general, a different performance model.

The template also includes parameters to define the workload intensity of the system, for instance the arrival rate of transactions from the environment, or the number of potential users to be supported. These parameters may be given a value or a range; a range implies the evaluation of performance over a set of values in that range.

\subsubsection{Modeling Software Component Interfaces}

Once we have software components, we need to model their interfaces so that we can hook them up with each other. These interfaces of these components would have two kinds of ports - input and output ports. Each port in a component interface has a list of service types or simply services associated with it.

Service types are descriptors that describe a service, not in complete and rigorous detail, but in sufficient detail for component selection. If alternatives are provided by the set of components, we assume that the interface details are designed either to be totally compatible, or to be adapted by the software generation process [8]. 
For an output port to be connected to an input port, all the required services specified by the first should be provided by the second. However, the input interface may provide services that are not used, so the services of the input port should be equal to or a superset of the set of services at the output port. Figure 3-4 illustrates this point.

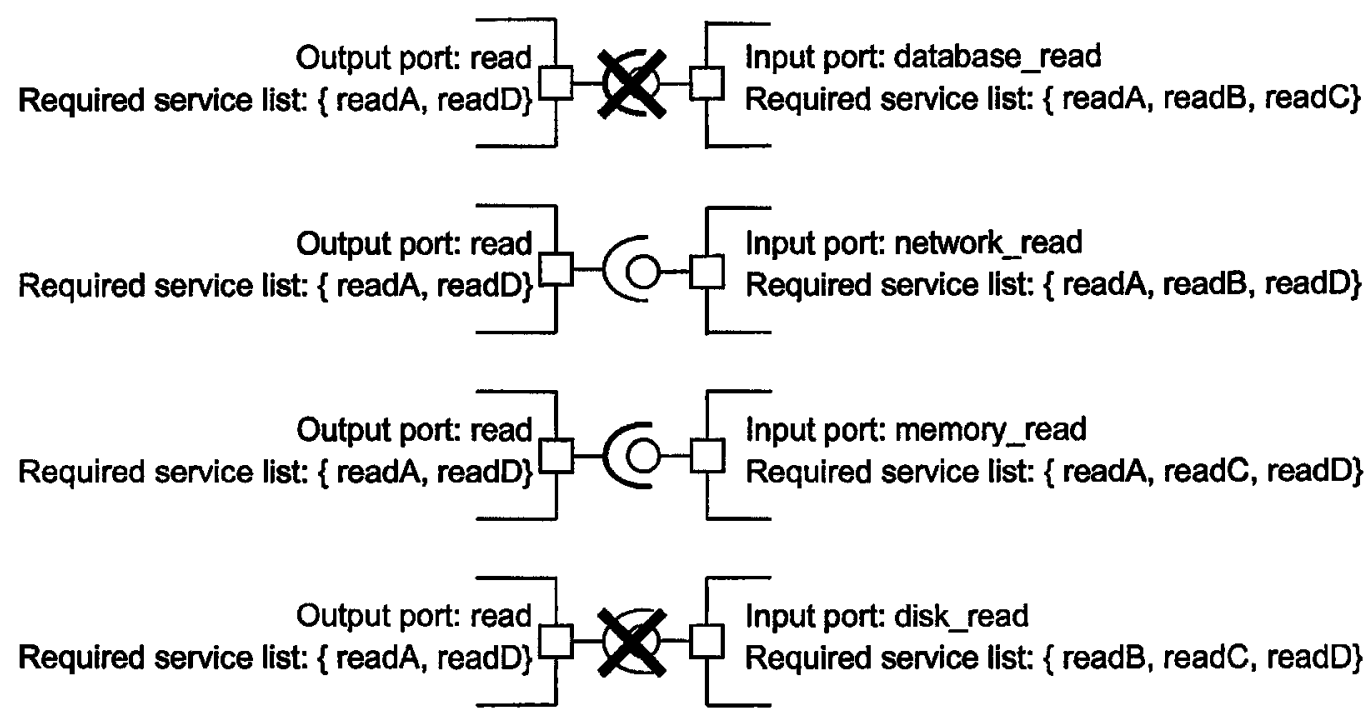

Figure 3-4: Principle of connecting a port by matching services

In the first and last cases of Figure 3-4, either readA or readD is not provided. The other cases provide readA and readD, and some additional service types that do not matter.

\subsubsection{Guiding Composition Process}

Composition constraints, other than provided or required service types, may arise from many different aspects of the software. To abstract these various aspects to a level 
suitable for performance models, components are described as belonging to groups which are mutually compatible (that is, similar enough to be used interchangeably, in aspects other than interface services). Groups are defined as set of components which share similar kind of functionality. A group has a name, and a component may be a member of any number of groups. A slot is defined with a list of permissible groups, and the groups are defined so as to exclude the possibility of using an unsuitable component.

Groups provide additional information about the functions provided by a component (in addition to the services provided by its interfaces) at the level of the entire component. Thus, a Database group can specify that a particular read-write interface is backed up by database functionality, and not just a file server. Clearly, the service types could provide a full description of the functionality of a component, however this approach is more flexible, and allows the use of rather general service types. The guiding principle is to cluster the components which provide the same or a similar set of services into one group and are likely to be used in similar kind of scenario. For example, you can put all the devices that store data and provide read and write access in a group called media. This might include a floppy disk controller, hard disk controllers, network interface etc.

The need for additional detail comes both from the requirement for functional compatibility, and from the need to limit the search space. Figure 3-5 shows an example of groups where 8 components are classified into 5 different groups. 


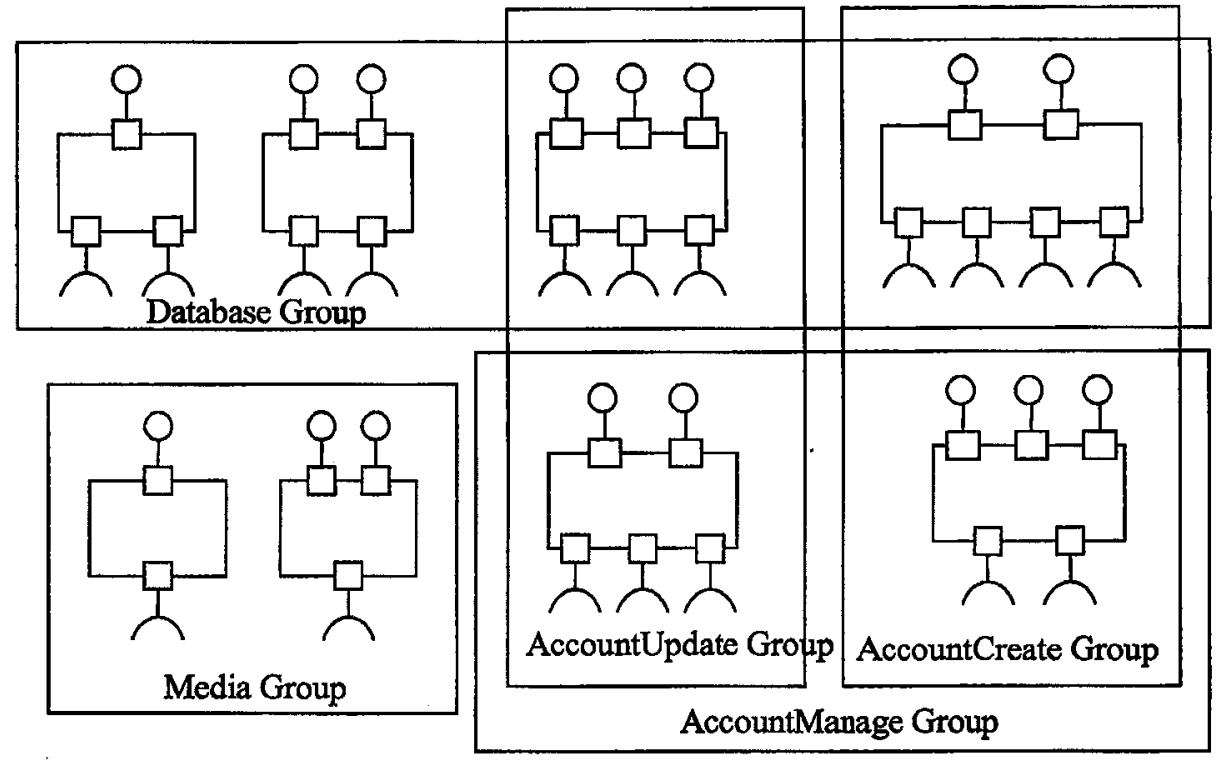

Figure 3-5: An example of classification of components in groups

\subsection{Evaluation and Ranking of Alternatives based on Requirements}

Performance goals are used to rank and screen the alternatives, after they are evaluated. They may range from strict requirements, to goals which are simply desirable. Strict requirements are expressed as hard constraints, which must be satisfied. Desirable or preferred requirements which may be violated to some degree are expressed as soft constraints, which have a "penalty of violation" parameter attached to them.

\subsubsection{Capturing Requirements though Constraints}

To formalize the treatment of constraints, a vector $x$ of all the performance measures for the system is defined, including the throughputs of different kinds of operations (operations/sec.), their mean response times (sec.), probabilities of violating a target response time (dimensionless), and the utilizations of various resources of the system. 
Then, without restriction of generality, the performance constraints may be expressed as a set of labeled inequalities of the form:

$$
f(x) \leq \text { target }\{\text { price }\}
$$

Here $f(x)$ is a performance constraint and target is the largest desirable value for $f(x)$, and the label \{price\} is non-zero for soft constraints only. Setting different prices for different constraints expresses their relative importance, and solutions will be ranked on their total penalty at these prices. The penalty for violation of a soft constraint is:

$$
\text { Penalty }=\text { price } \times[(f(x)-\text { target }) / \text { target }]^{+}
$$

Here the superscript ${ }^{+}$indicates that the bracket is replaced by zero if it is negative, as shown in Figure 3-6.

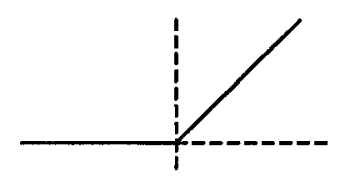

Figure 3-6: A plot for $y=[x]^{+}$

This work has used linear functions for $f(x)$, but they may equally be nonlinear. The two constraints given below in throughputs $\mathrm{F}$ are expressed as greater-than requirements, but they can easily be put into canonical form (with $\leq$ ) by multiplying through by -1 . Also the constraints can also be expressed as sums which represent trade-offs that 
combine two measures. The evaluation of a set of alternative configurations divides them into three groups:

- configurations that meet all performance constraints,

- those that meet all the hard constraints but violate one or more soft constraints, ranked by total penalty

- those that do not meet one or more of the hard constraints

\subsubsection{Hard Constraints}

Hard constraints here are those that must be met. To express a hard real-time constraint one could impose a hard constraint on a percentile measure with zero violations allowed, as follows:

$$
\text { Probability }\{\mathrm{R}>2 \mathrm{~ms}\} \leq 0
$$

Hard constrains for our online banking example is shown in Table 3-1. The notation used for expressing these constraints is the same as explained in Section 3.2, and is based on mean values for quantities.

\section{Hard Constraints}

(1) $R_{\text {Check_Balance_UI }} \leq 2 \mathrm{sec}$

(2) Fonline_Payment_ur $\geq 15 /$ sec

(3) $5 \mathrm{~F}_{\text {Transfer_Cash_UI }}+$ Fonline_Payment_Ur $_{-} \geq 100 / \mathrm{sec}$

Table 3-1: Hard constraints for online banking example. 


\subsubsection{Soft Constraints}

A soft real-time constraint could similarly be expressed by a hard constraint on a percentile measure, for instance at the $5 \%$ level, as:

$$
\text { Probability }\{\mathrm{R}>2 \mathrm{~ms}\} \leq 0.05
$$

Table 3-2 shows an example of constraints to represent performance requirements. The constraints involve only the mean response times and utilization of devices.

\begin{tabular}{ll}
\hline \multicolumn{2}{c}{ Soft Constraints } \\
\hline (4) $\mathrm{U}_{\mathrm{NFS}} \leq 35 \%$ & $\{5\}$ \\
(5) $2 \mathrm{~F}_{\text {Transfer_Cash_UI }}+$ Fonline_Payment_UI $_{2} \geq 60 / \mathrm{sec}$ & $\{2\}$ \\
(6) $2 \mathrm{R}_{\text {Transfer_Cash_UI }}+5 \mathrm{R}_{\text {Online_Payment_UI }} \leq 20 \mathrm{sec}$ & $\{3\}$ \\
\hline
\end{tabular}

Table 3-2: Soft constraints for online banking example with penalty prices in brackets \{\}

Different prices are shown for the soft constraints, indicating the different importance attached to violation of each one. Prices may be rough measures of importance, or could be derived from business data relating to the cost of delay or of inadequate capacity.

\subsubsection{Evaluating Suitable Alternatives}

Once all the possible alternative configurations are enumerated, we still need to evaluate them to see their fitness to our requirements. Thus, only those alternatives that satisfy the performance goal are selected and evaluated to give a mismatch parameter that measures 
how closely the configuration matches with the desired features. The less the mismatch parameter is, the better our obtained configuration is from a performance point of view. For each alternative $i$, the penalty is defined as:

$$
\text { penalty }_{i}=\sum_{j=1}^{M} \text { penalty }_{-} \text {price }_{j} \times\left[\left(f_{j}\left(x_{i}\right)-\text { target }_{j}\right) / \text { target }_{j}\right]^{+}
$$

where:

- penalty $_{i}$ is the total penalty for the $i^{\text {th }}$ alternative, and $x_{i}$ is its vector of performance measures,

- penalty price $_{j}$ is the price of the $j^{\text {th }}$ soft constraint, out of $M$ soft constraints

Soft constraints are normalized for evaluation. This places all the constraints at equal weight relative to each other. This is necessary because the relative weight of constraints is controlled by user through penalty of constraints and it should be not dependent on parameters present in the constraint.

\subsection{An Example}

The template model for the online banking example of Figure 3-3 is shown in Figure 3-7. The source actors for the three different types of request are modeled as three driver tasks namely, Client1, Client2, and Client3. The User_Interface task is a component, specific to the template, with an entry for each operation which models the workload. There are three slots for an Application server, a Database server, and a File server respectively. Each slot provides alternatives, which can be combined to give a number of alternatives 
over all the configurations to be explored. DBP, NFS and MainP are the host processors which would replace the replaceable processors defined in slots Database, Update_Account and File_Server.

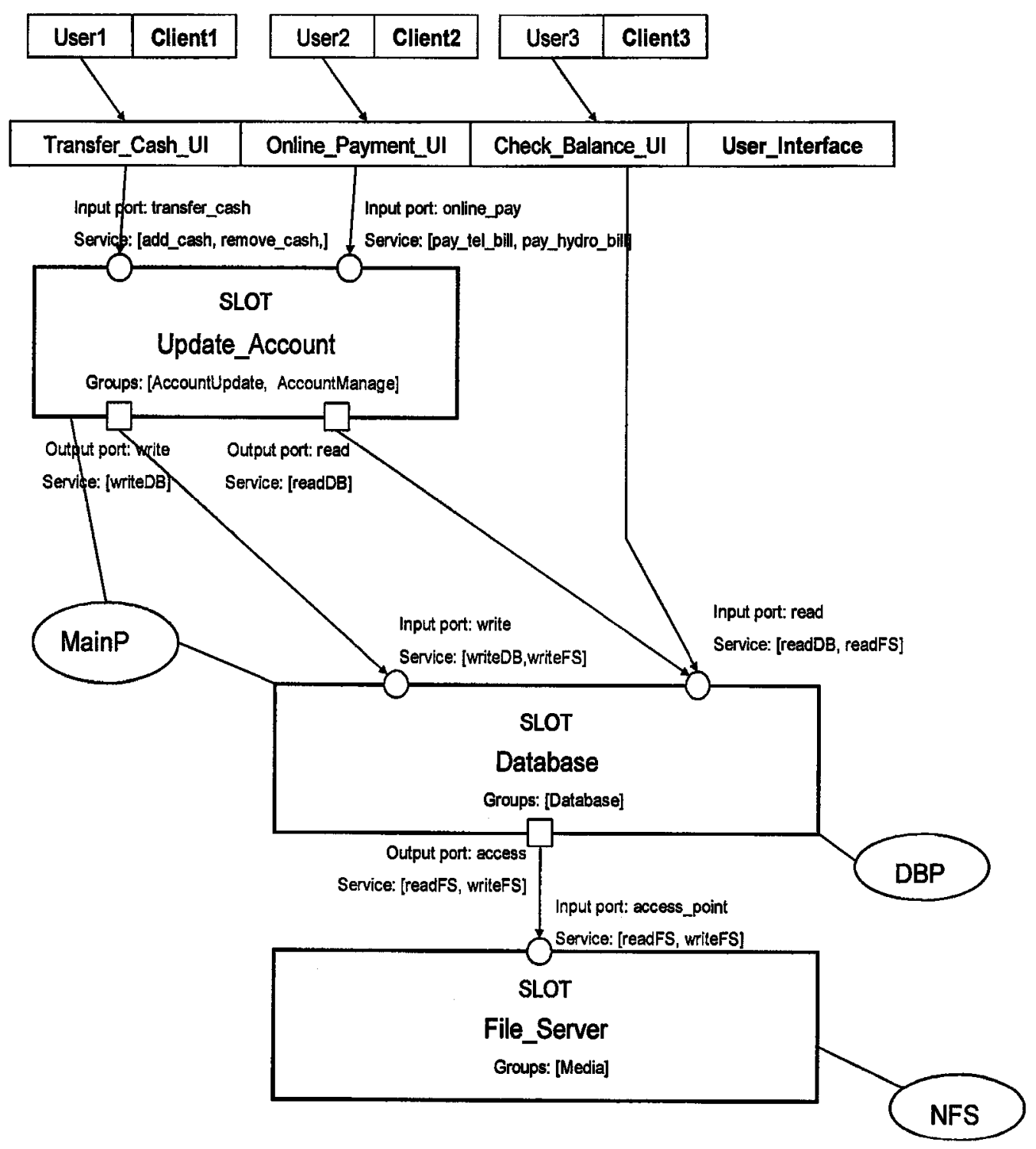

Figure 3-7: A template model in CBML for online banking example with three slots 
The template shown in Figure 3-7 has three slots - Database, File_Server, and Update_Account. There are six components that can fill these slots, as determined by matching the interface types and groups. The CBML models are shown in Appendix A. They are summarized in Table 3-3.

\begin{tabular}{c|l|l|l}
\hline $\begin{array}{l}\text { Component } \\
\text { name }\end{array}$ & Slot name & Groups & Description \\
\hline F & File_Server & Media, Storage & A simple file server \\
\hline D1 & Database & $\begin{array}{l}\text { Database, } \\
\text { Storage }\end{array}$ & $\begin{array}{l}\text { A simple database component } \\
\text { providing basic read and write } \\
\text { operation. }\end{array}$ \\
\hline D2 & Database & $\begin{array}{l}\text { Database, } \\
\text { Storage }\end{array}$ & $\begin{array}{l}\text { A database component having a } \\
\text { cache for read operation. }\end{array}$ \\
\hline M1 & Database & DBMS & $\begin{array}{l}\text { A database component optimized } \\
\text { for read operations. }\end{array}$ \\
\hline M2 & Update_Account & AccountUpdate & $\begin{array}{l}\text { Account managing task that uses a } \\
\text { common base task for adding and } \\
\text { removing cash. }\end{array}$ \\
\hline
\end{tabular}

\section{Table 3-3: Description of the six components}

Using the tool, six different alternative configuration - F:D1:M1, F:D1:M2, F:D2:M1, F:D2:M2, F:D3:M1, and F:D3:M2 - are generated where F:Dx.Mx means that template is filled with component $\mathrm{F}$ in slot File_Server, Component Dx in slot Database and component $\mathrm{Mx}$ in Update_Account Slot. 
The six configurations that are described above were constructed and evaluated for constraints described in Table 3-1 and 3-2 with numbers of active users $(\mathrm{N})$ varied from 10 to 50 . The user delay between transactions which models the reaction time to read the output and enter the next transaction was set to $2 \mathrm{sec}$. These CBML models were solved [37] to obtain the performance parameters for range of users $(\mathrm{N})$ varying from 10 to 50 in steps of 5. The results were screened by the hard constraints. Figures 3-8 though Figure 3-16 show the effect of the constraints on the alternatives. Four alternatives - F:D1:M2, F:D2:M1, F:D2:M2, and F:D3:M2, satisfied all three hard constraints. The final constraints were evaluated at $\mathrm{N}=50$.

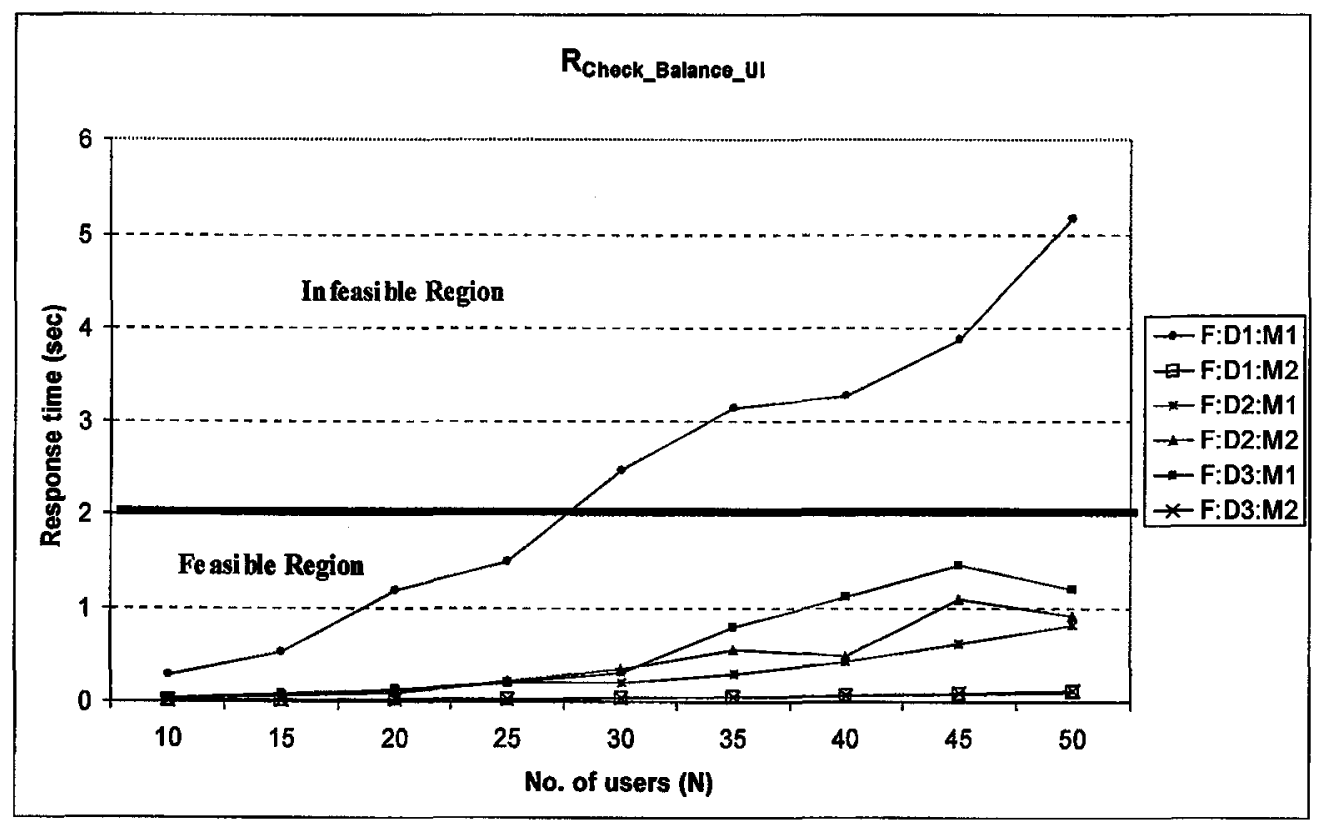

Figure 3-8: Plot of the function $f(x)$ for hard constraint (1) in Table 3-1 showing feasible and infeasible areas of response times. 


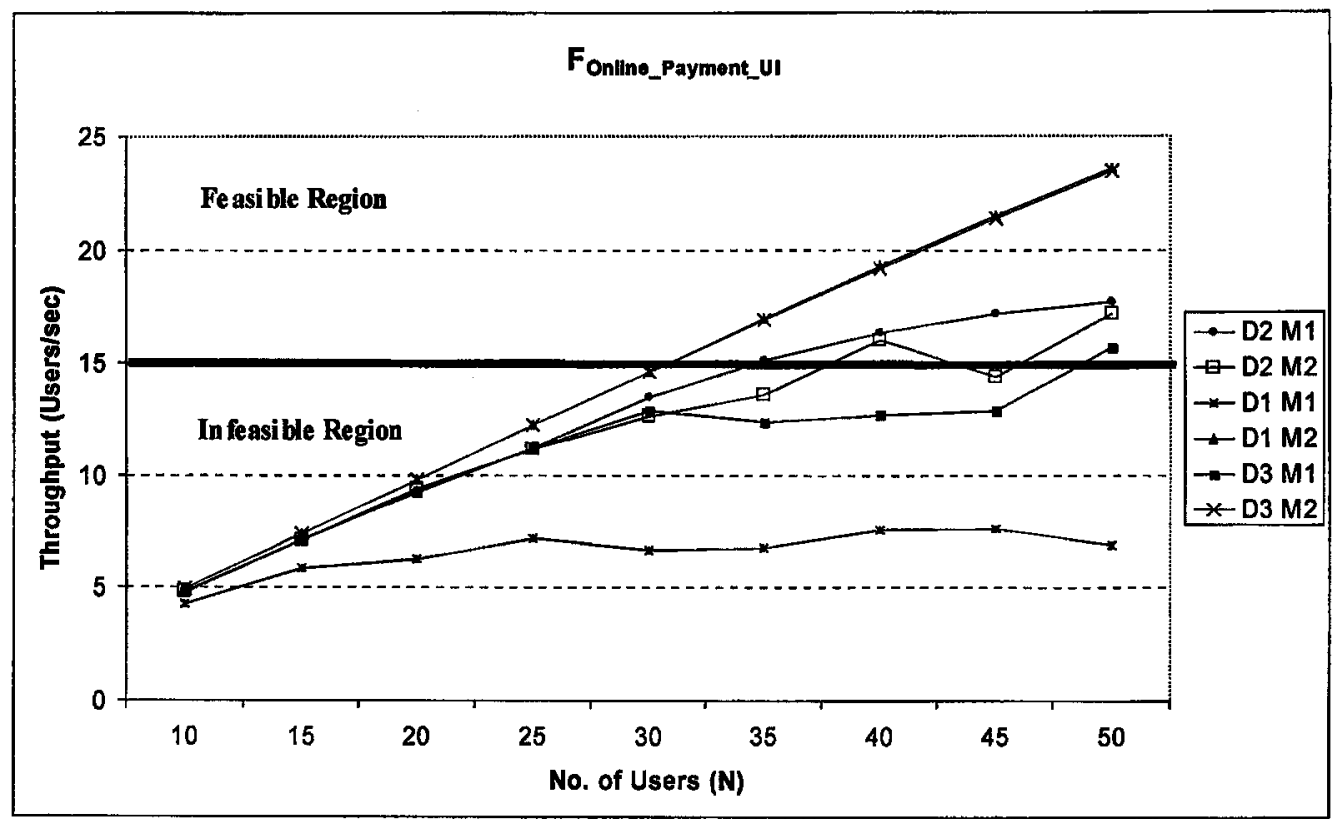

Figure 3-9: Plot of the function $f(x)$ for hard constraint (2) in Table 3-1 showing feasible and unfeasible areas of response times.

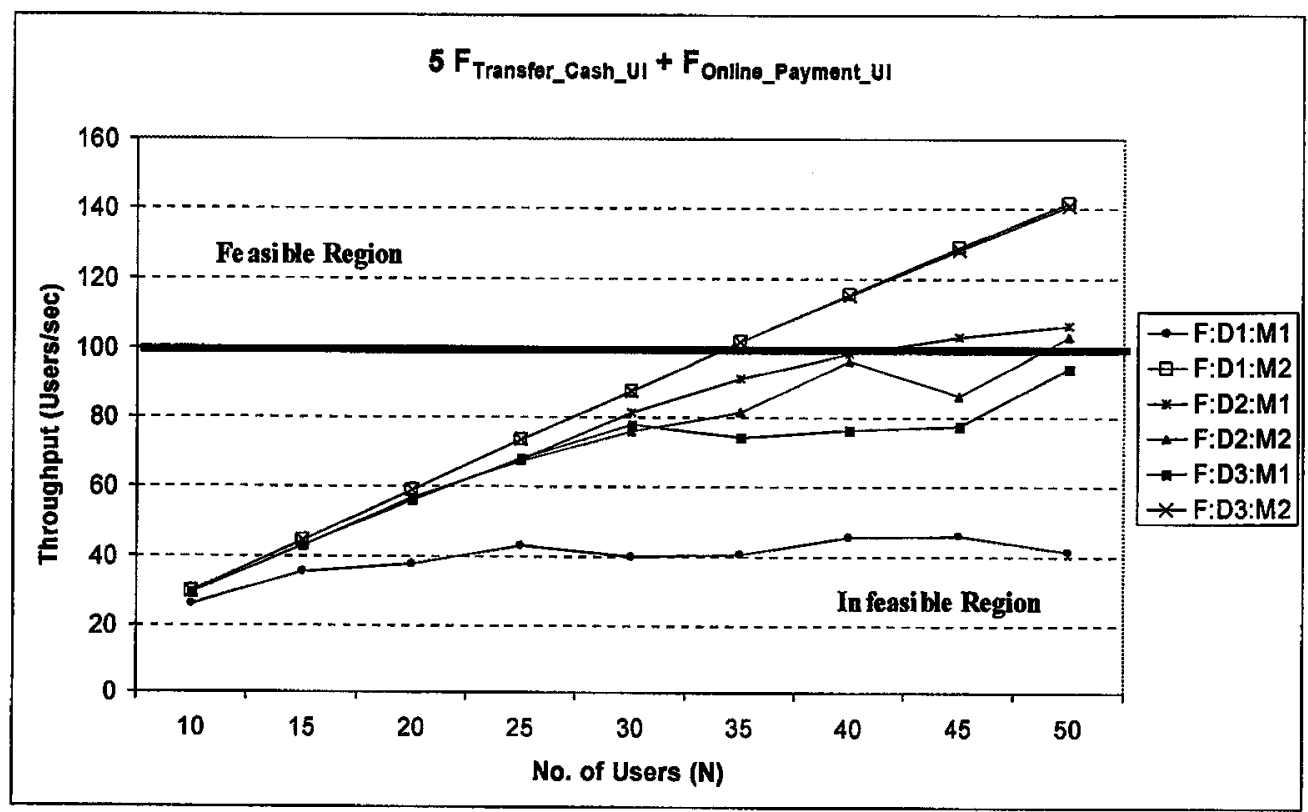

Figure 3-10: Plot of the function $f(x)$ for hard constraint (3) in Table 3-1 showing feasible and infeasible areas of response times. 


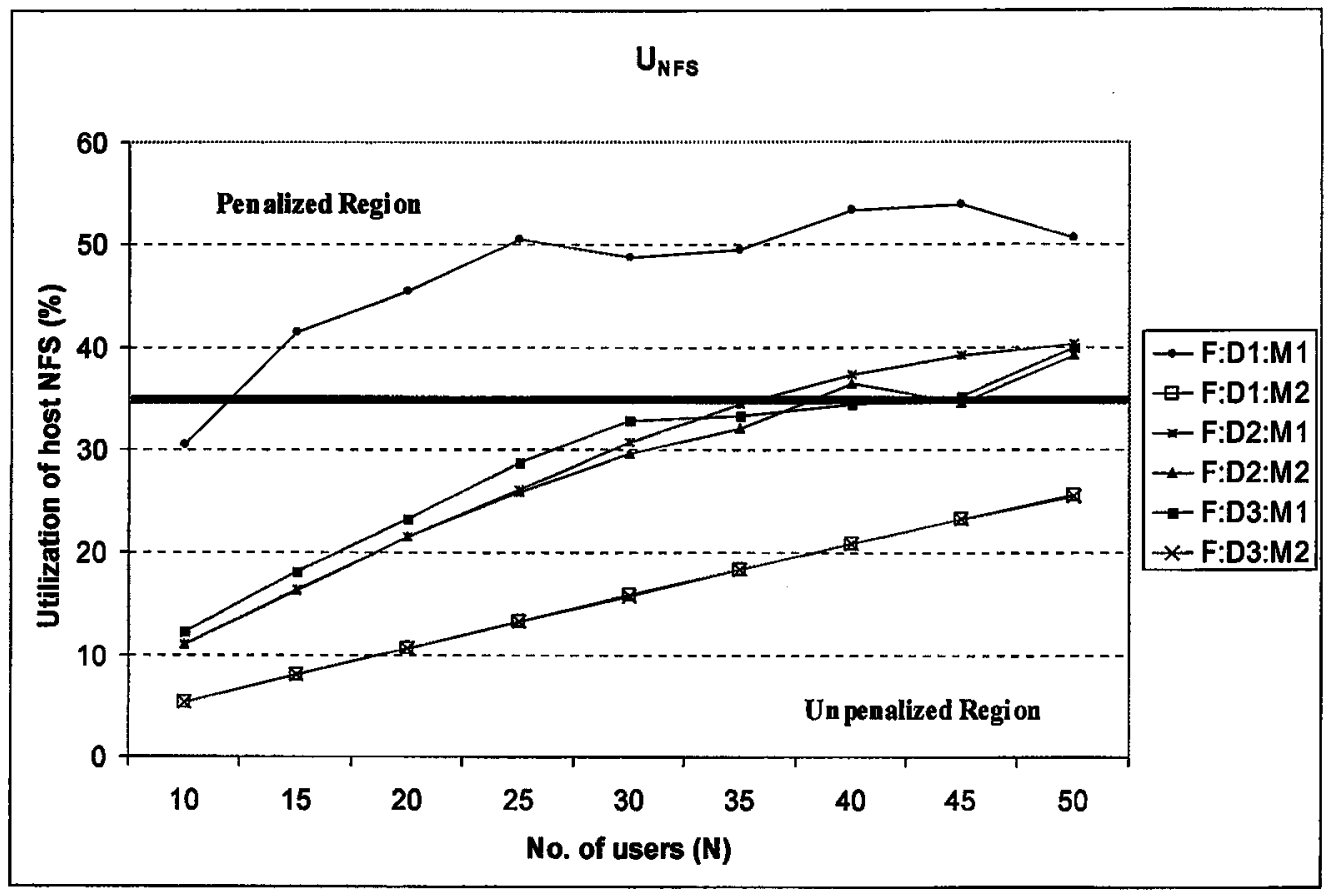

Figure 3-11: Plot of the function $f(x)$ for soft constraint (1) in Table 3-2, showing penalized and unpenalized regions.

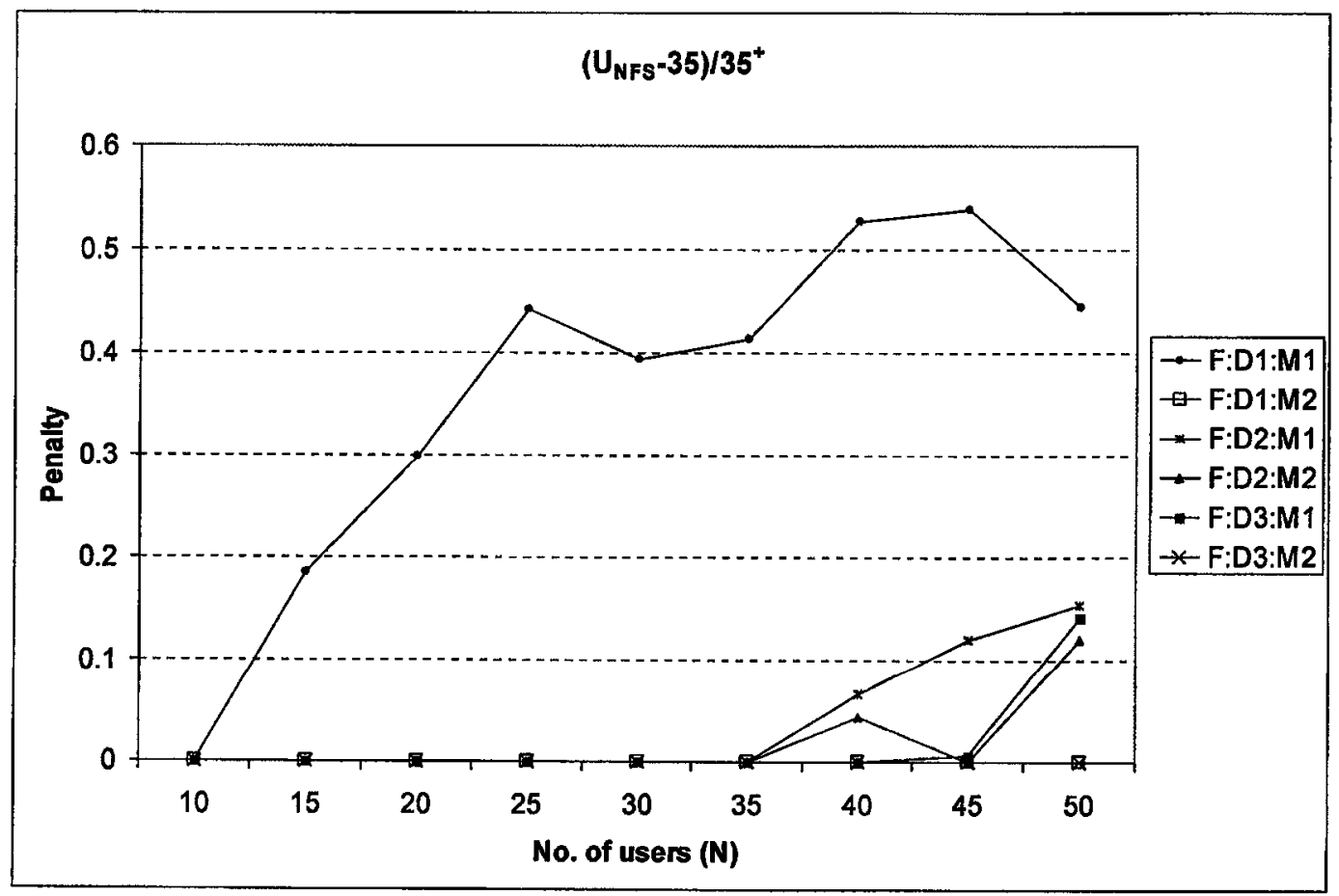

Figure 3-12: Plot of the penalty for soft constraint (1) in Table 3-2 


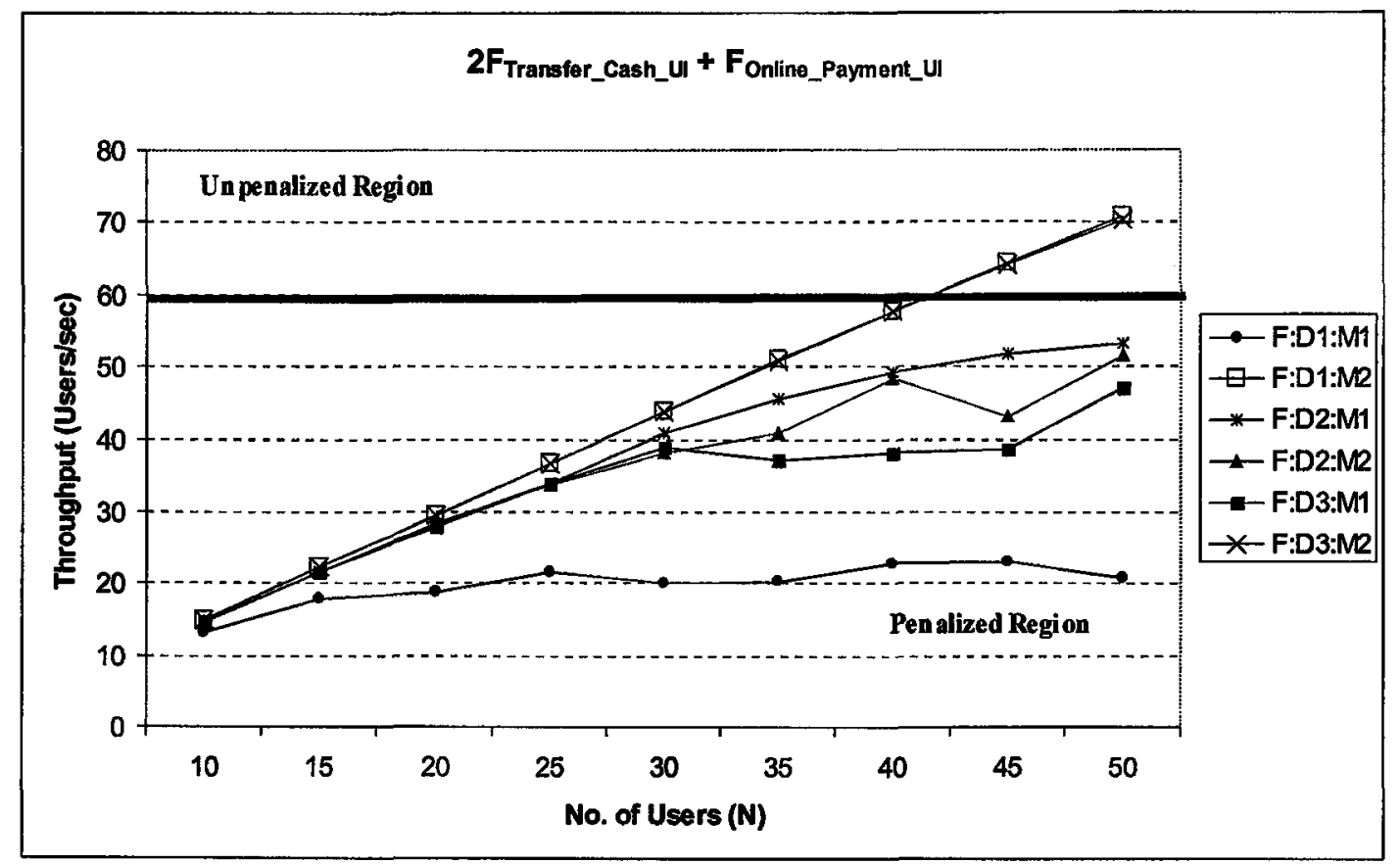

Figure 3-13: Plot of the function $f(x)$ for soft constraint (2) in Table 3-2, showing penalized and unpenalized regions.

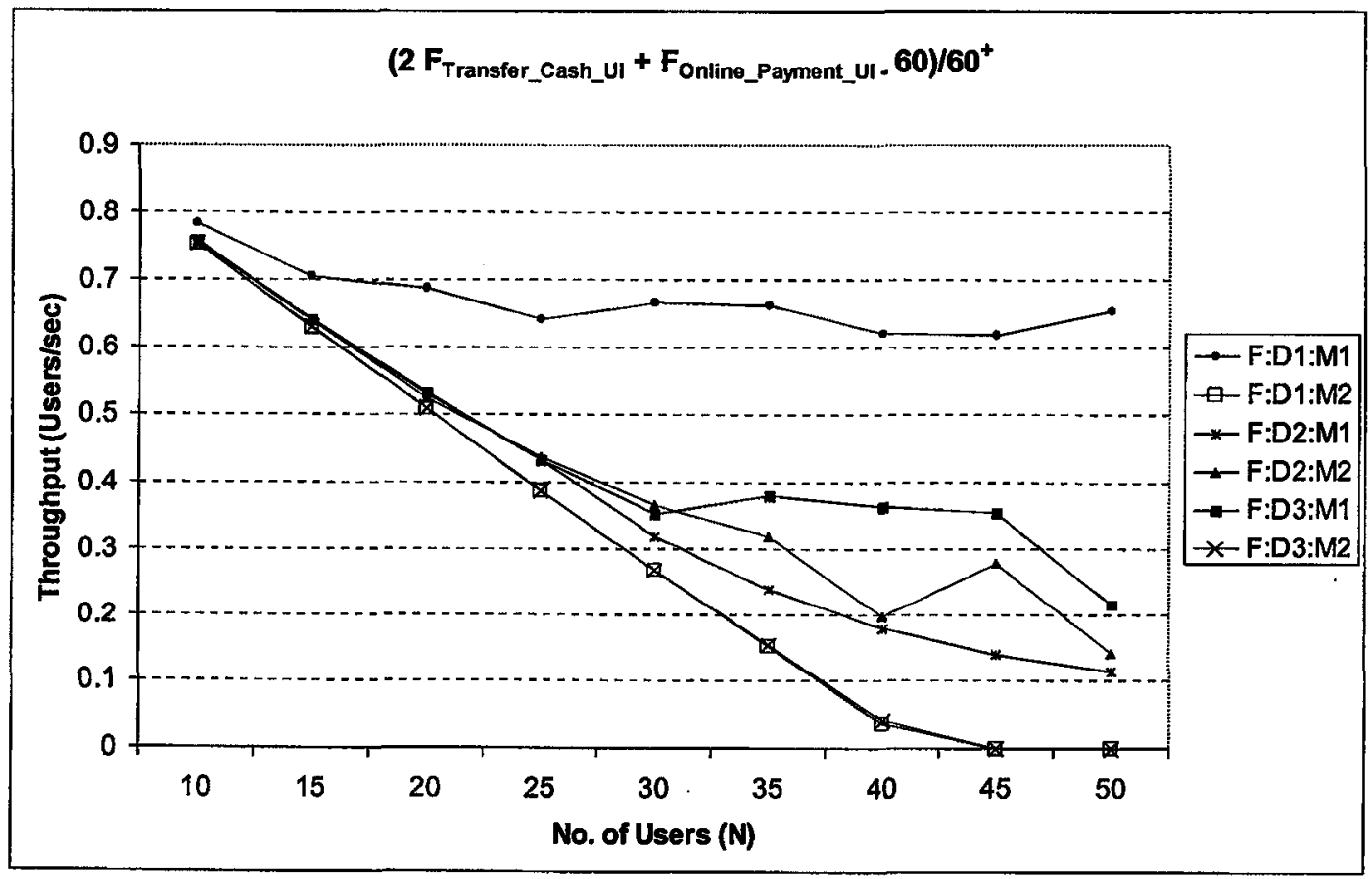

Figure 3-14: Plot of the penalty for soft constraint (2) in Table 3-2 


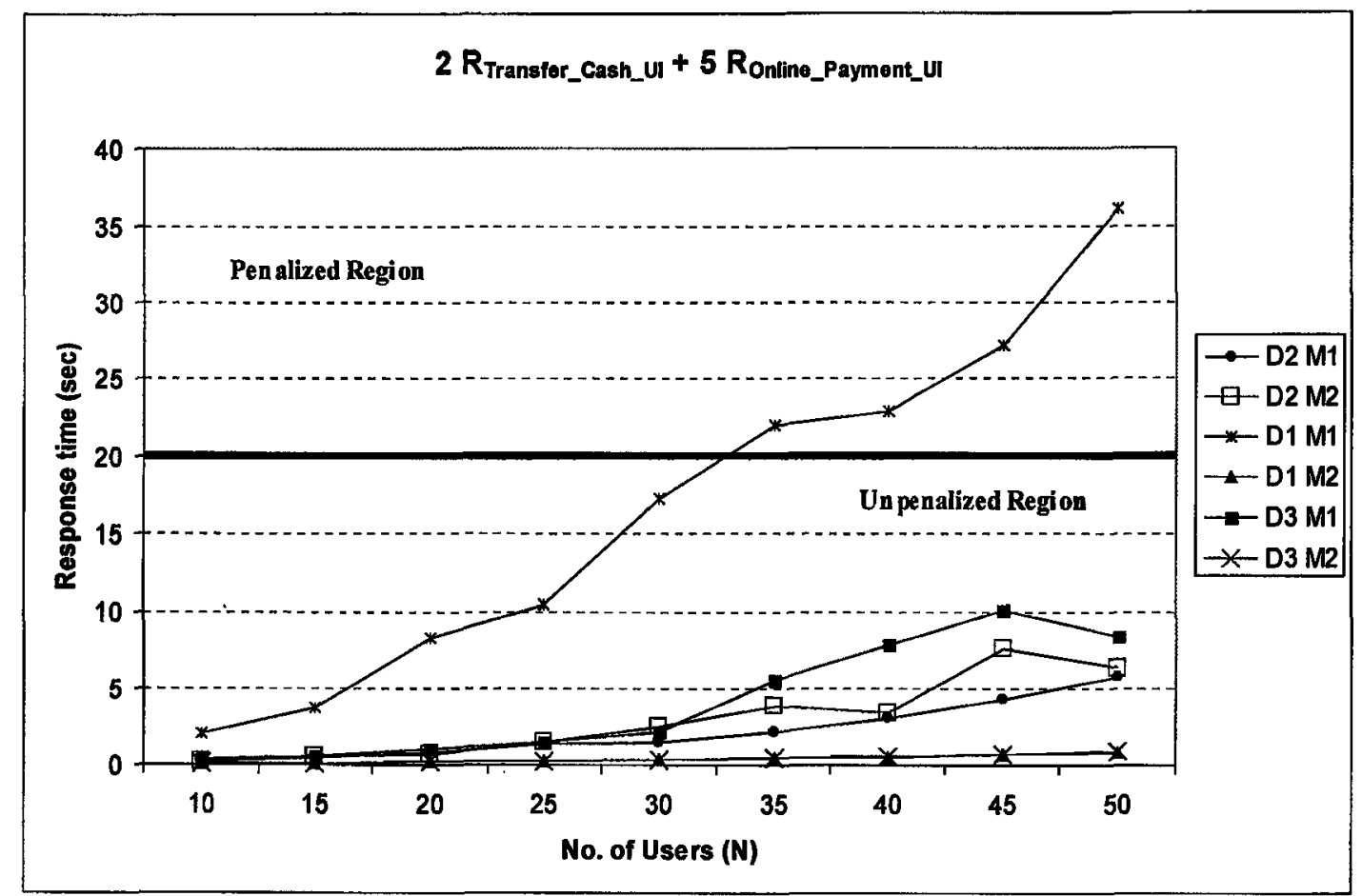

Figure 3-15: Plot of the function $f(x)$ for soft constraint (3) in Table 3-2, showing penalized and unpenalized regions.

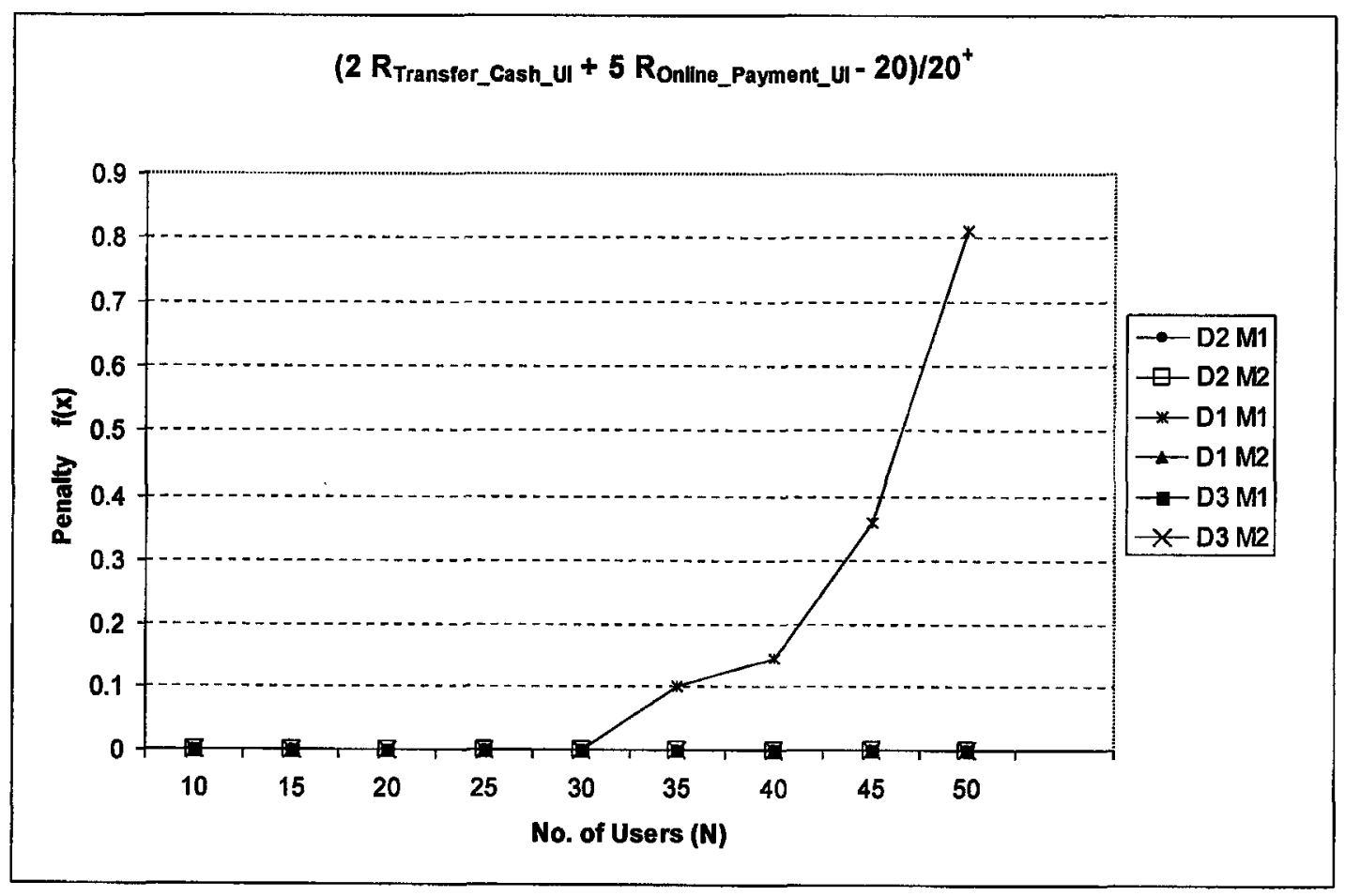

Figure 3-16: Plot of the penalty for soft constraint (3) in Table 3-2 


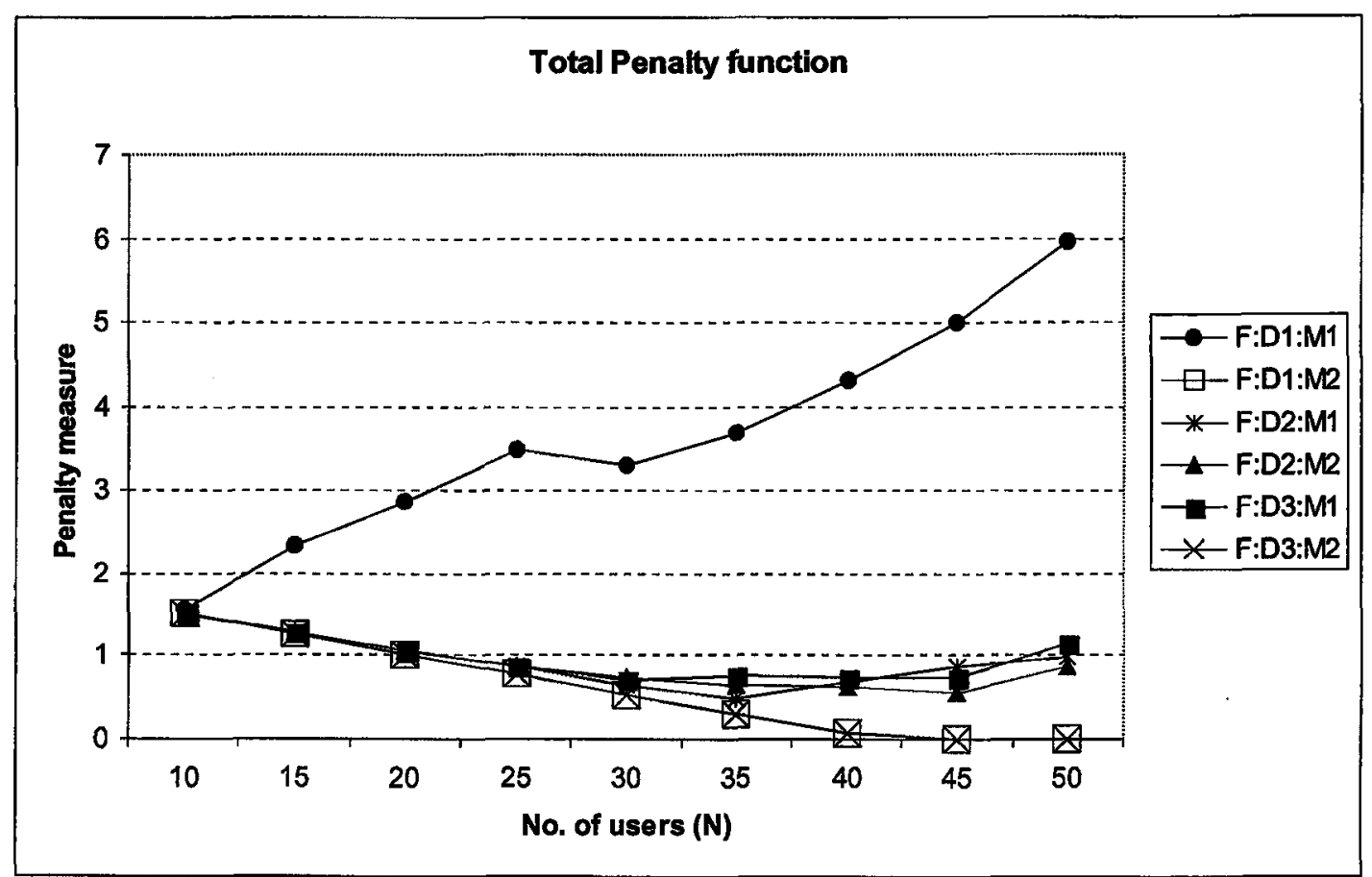

Figure 3-17: Plot of total penalty for all constraints shown in Table 3-2

It should be noted that the value at $y$-axis of penalty curves of Figure 3-14 decreases by increasing $\mathrm{N}$ whereas penalty curves of Figure 3-12 and Figure 3-16. This is because the first and the third constraints are of the form $f(x)<d$ whereas the second constraints is of the form $f(x)>d$. The total penalty value for the four alternatives is calculated for the entire range of $\mathrm{N}$ and is shown in Figure - 3.17.

The penalty measures at $\mathrm{N}=50$ is taken for evaluating the four alternatives that satisfy the hard constraints. It should be noted that first and third term are of the form $f(x)-d$ and the second term is of the form $d-f(x)$. This is because the first and the third constraints are of the form $f(x)<d$ whereas the second constraints is of the form $f(x)>d$.

For $\mathrm{F}: \mathrm{D} 1: \mathrm{M} 2$, at $\mathrm{N}=50$, the value of constraints are: 
$\mathrm{U}_{\mathrm{NFS}}=25.63$

$2 \times F_{\text {Transfer_Cash_UI }}+F_{\text {Check_Balance_UI }}=70.81$

$2 \times \mathrm{R}_{\text {Transfer_Cash_UI }}+5 \times \mathrm{R}_{\text {Check_Balance_UI }}=0.8$

The total penalty measure is:

$$
\begin{aligned}
& \text { penalty }=5 \times[(25.63-35) / 35]^{+}+2 \times[(60-70.81) / 60]^{+}+3 \times[(0.8-20) / 20]^{+} \\
& =5 \times[-0.359]^{+}+2 \times[-0.180]^{+}+3 \times[-0.96]^{+}=0
\end{aligned}
$$

For $\mathrm{F}: \mathrm{D} 2: \mathrm{M} 1$, at $\mathrm{N}=50$, the value of constraints are:

$$
\begin{aligned}
& \mathrm{U}_{\mathrm{NFS}}=40.38 \% \\
& 2 \times \mathrm{F}_{\text {Transfer_Cash_UI }}+\mathrm{F}_{\text {Check_Balance_UI }}=53.18 \\
& 2 \times \mathrm{R}_{\text {Transfer_Cash_UI }}+5 \times \mathrm{R}_{\text {Check_Balance_UI }}=5.74
\end{aligned}
$$

The total penalty measure is:

$$
\begin{aligned}
& \text { penalty }=5 \times[(40.38-35) / 35]^{+}+2 \times[(60-53.18) / 60]^{+}+3 \times[(5.74-20) / 20]^{+} \\
& =5 \times[0.154]^{+}+2 \times[0.114]^{+}+3 \times[-.713]^{+}=0.996
\end{aligned}
$$

For $\mathrm{F}: \mathrm{D} 2: \mathrm{M} 2$, at $\mathrm{N}=\mathbf{5 0}$, the value of constraints are:

$$
\begin{aligned}
& \mathrm{U}_{\mathrm{NFS}}=39.18 \\
& 2 \times \mathrm{F}_{\text {Transfer_Cash_UI }}+\mathrm{F}_{\text {Check_Balance_UI }}=51.59 \\
& 2 \times \mathrm{R}_{\text {Transfer_Cash_UI }}+5 \times \mathrm{R}_{\text {Check_Balance_UI }}=6.35
\end{aligned}
$$

The total penalty measure is: 


$$
\begin{aligned}
& \text { penalty }=5 \times[(39.18-35) / 35]^{+}+2 \times[(60-51.59) / 60]^{+}+3 \times[(6.35-20) / 20]^{+} \\
& =5 \times[0.119]^{+}+2 \times[0.14]^{+}+3 \times[-.682]^{+}=0.877
\end{aligned}
$$

For $\mathrm{F}: \mathrm{D} 3: \mathrm{M} 2$, at $\mathrm{N}=50$, the value of constraints are:

$$
\begin{aligned}
& \mathrm{U}_{\mathrm{NFS}}=25.49 \\
& 2 \times \mathrm{F}_{\text {Transfer_Cash_UI }}+\mathrm{F}_{\text {Check_Balance_UI }}=70.42 \\
& 2 \times \mathrm{R}_{\text {Transfer_Cash_UI }}+5 \times \mathrm{R}_{\text {Check_Balance_UI }}=0.88
\end{aligned}
$$

The total penalty measure is:

$$
\begin{aligned}
& \text { penalty }=5 \times[(25.49-35) / 35]^{+}+2 \times[(60-70.42) / 60]^{+}+3 \times[(0.88-20) / 20]^{+} \\
& =5 \times[-0.362]^{+}+2 \times[-0.173]^{+}+3 \times[-0.956]^{+}=0
\end{aligned}
$$

\begin{tabular}{l|l}
\hline Alternative & Penalty measure \\
\hline F:D1:M1 & Rejected by hard constraint \\
F:D1:M2 & 0 \\
F:D2:M1 & 0.996 \\
F:D2:M2 & 0.877 \\
F:D3:M1 & Rejected by hard constraint \\
F:D3:M2 & 0 \\
\hline
\end{tabular}

Table 3-4: Summary of penalty values for all alternatives at $\mathbf{N}=\mathbf{5 0}$

Figure 3-17 shows that penalties for low throughput at small numbers of users dominate (small $N$ ), and these decrease with $N$. For some alternatives the penalty for large response time takes over and increases with $N$. The second and sixth alternatives 
become penalty-free for $N$ above 40 , and they are also the best choices (smallest penalty) for smaller $N$. The penalty measures for all alternatives are summarized in Table 3-4.

From this data the preferred alternatives at $N=50$ are F:D1:M2 and F:D3:M3 with no preference between them, followed by F:D2:M2, and finally F:D2:M1. The actual choice can be influenced by the values at different $N$ and by additional factors such as estimated cost or maintainability.

\subsection{Conclusion}

The example shows how the scheme is simple yet effective. It is easy to express tradeoffs in linear forms, and to express relative desirability as prices of violation of the soft constraints. It is easy to trace back to the requirements. If additional factors are considered, one can adjust the priority and violation parameters to resolve conflicts. If it were desired to extend it, any sort of non-linear inequality and penalty function could also be used. 


\section{Chapter 4: Library Manager Tool}

The framework described here for modeling systems can be quite cumbersome to use by hand. Each binding of component in a slot needs to satisfy constraints like group matching for the slot as well as service matching for all the input/output ports of components. Further enumeration is needed in certain cases, which can be a tedious job to be done manually.

Library manager tool is developed to automate these tasks. The objective of the Library Manager Tool is to assist user to decide which component should be bound, keeping all constraints, and to automatically generate all the alternatives where necessary. This chapter discusses the design and use of the Library Manager Tool.

\subsection{Overview of the Library Manager Tool}

The basic objective of the tool is to maintain libraries of components and use them to build components. Each library would have the following information:

- List of components with pointers to their CBML models (XML files).

- List of groups and the names of their member components.

- Information for each slot of each component containing list of groups who members can be bound to that particular slot. 
- Information for all ports containing the list of services required or provided by that port.

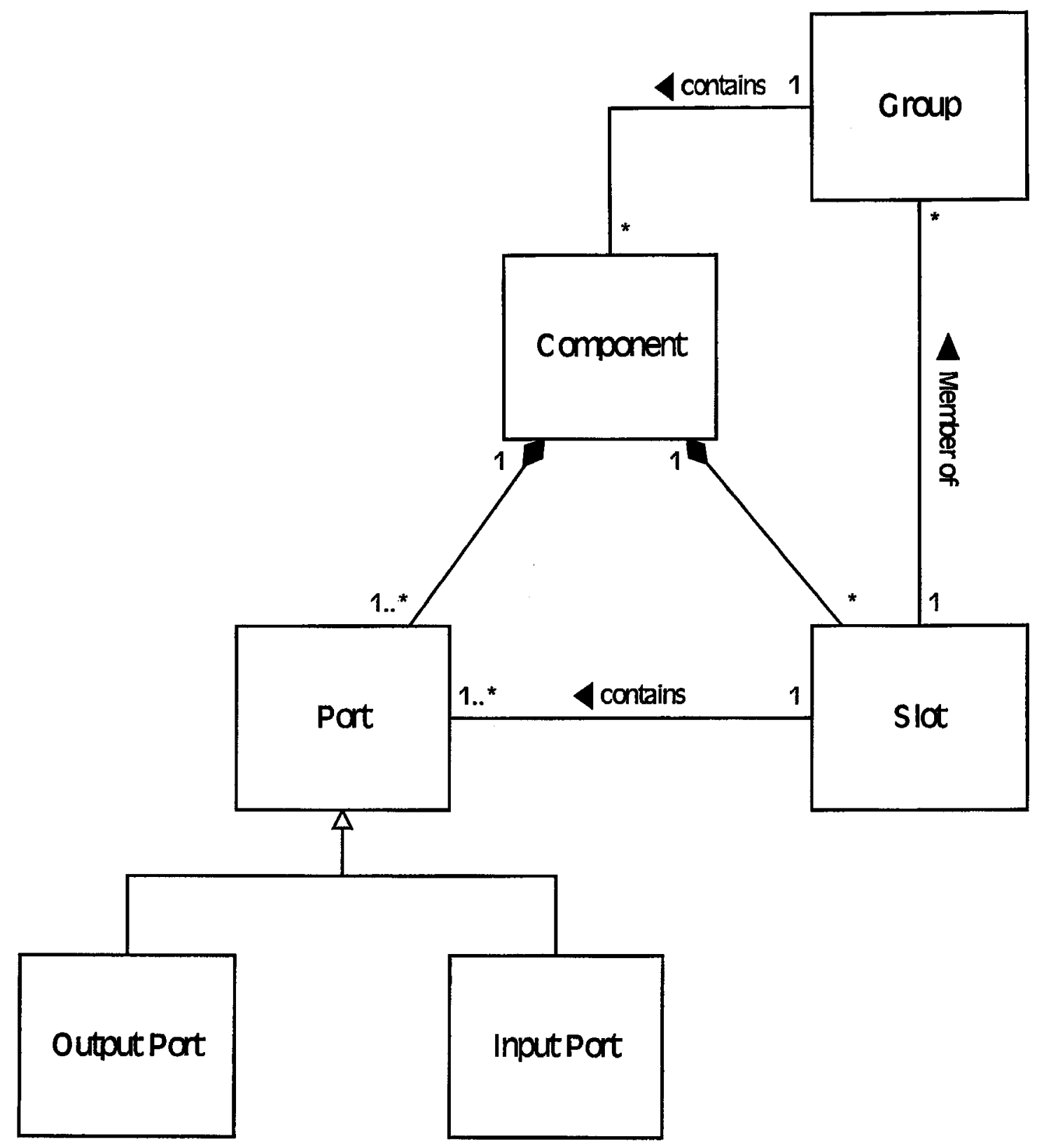

Figure 4-1: UML relationship diagram for various elements of modeling framework 
Thus components, slots and ports are the basic elements of the modeling environment and their relationship in described in Figure 4-1 in form of UML diagram. It should be noted that this is a conceptual diagram and does not exactly correspond with the static class diagram of the implementation.

\subsection{Managing Components with the Library Manager Tool}

This section briefly describes the various control panels of the tools that are used to manage components. The main screen is divided into two panes - Groups pane and the Components pane. A screen shot of the group pane is shown in Figure 4-2 and contains a list of groups in the library along with the buttons to manipulate them. A screen shot of component pane is shown in Figure 4-3, which contains the list of components along with buttons to manipulate them and their slots and ports. The title of the window contains the name of the library file loaded (in case of Figure 4-2 and Figure 4-3, it is test.lib). By default, when the tool is started, there is no library loaded and hence title does not mention any file. There are five buttons on top of these panes. These are:

- Create Lib: This will clear the currently loaded library. Clearing of the library implies that all information is erased. This will results in a state where there are no groups in the group list and no component is loaded in the component list.

- Load Lib: This loads a new library file from disk. The existing library is removed without warning. 
- Merge Lib: This would load another library on top of the currently loaded one. Where a conflict between components/groups of the two libraries happen, priority is given to the old one.

- Save Lib: This option is used for saving library to a file on disk.

- Exit: This option shuts down and exits the tool.

\subsubsection{Group Controls}

The group pane shown in Figure 4-2 is the main pane for controlling groups. Groups can be created, edited (i.e. Renamed) or deleted here. It has a text field for entering and editing the names of groups.

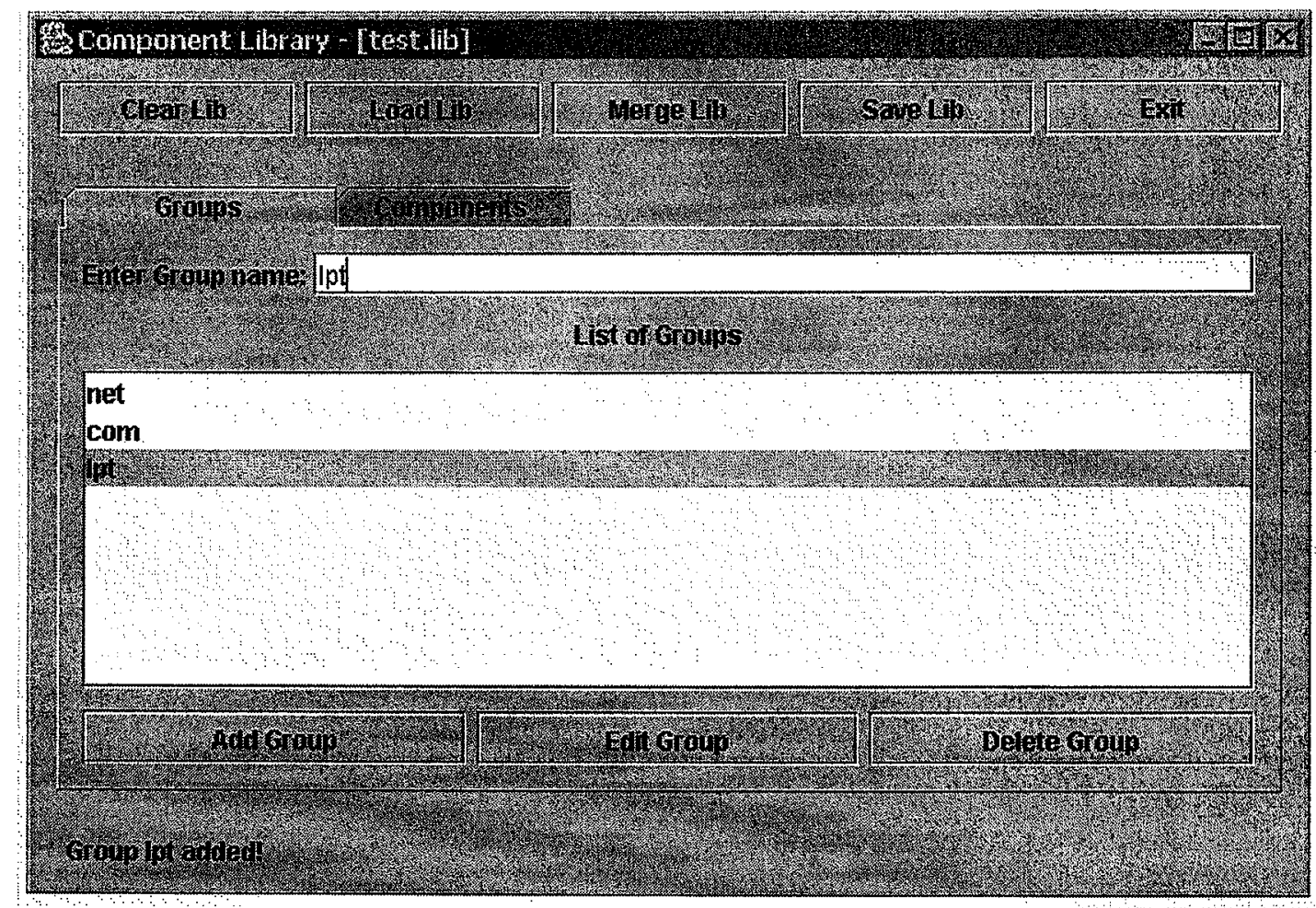

Figure 4-2: Main panel with Groups pane active 
The group control panel has three buttons which can be used for editing group. The "Add Group" option would create a new group with no members. The new group name needs to be entered in the text field. The "Edit Group" option would change the name of the selected group. The new name has to be entered in the text field. This will only work if there is no clashing of names among groups and the change is reflected in all member components of the group. The "Delete Group" option would delete the selected group but the member components are retained in the component list.

\subsubsection{Component Controls}

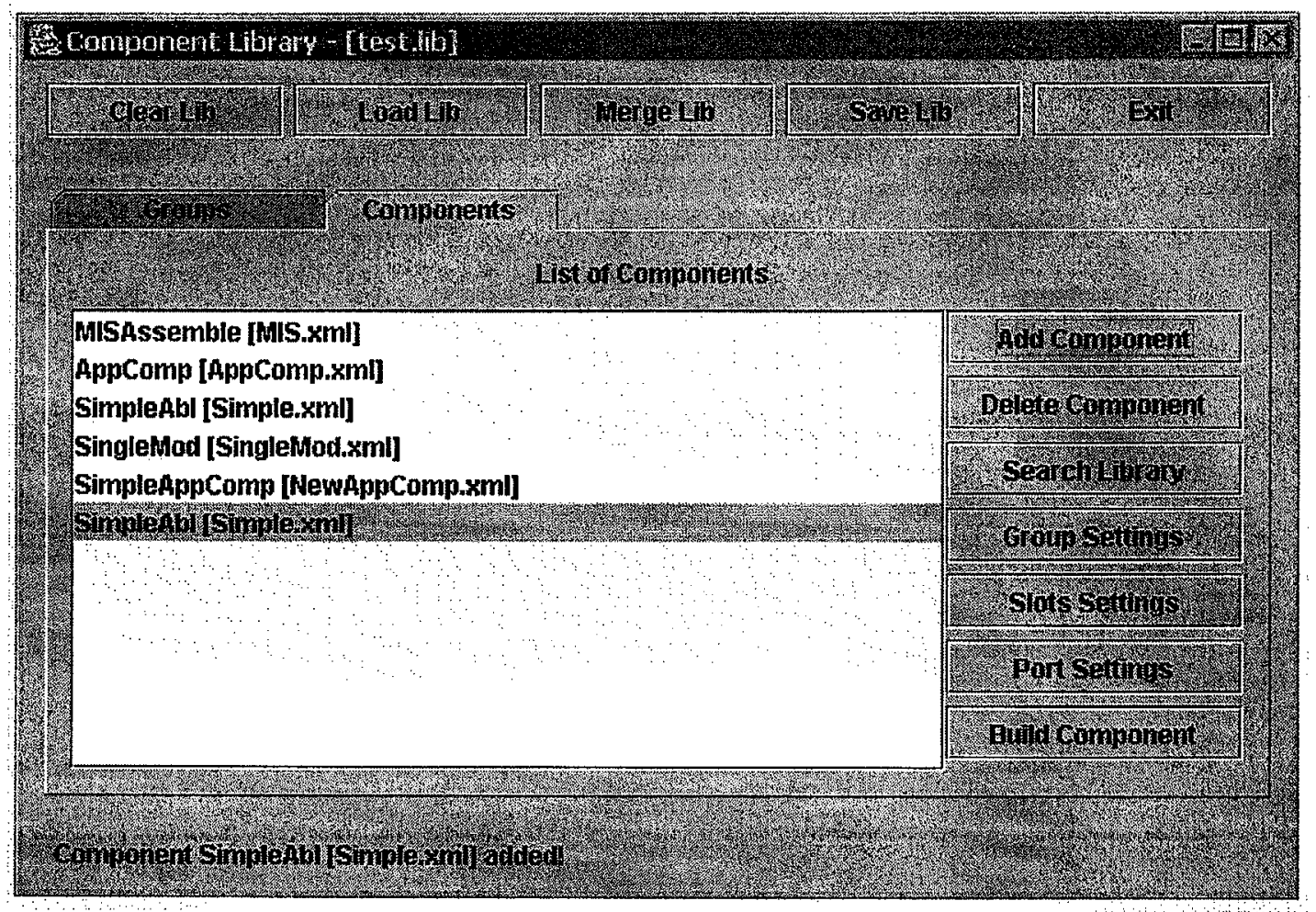

Figure 4-3: Main panel with Component pane active 
Figure 4-3 shows the main panel with component pane active. Various operations on components are possible through the buttons present on the right side of the list. Add component adds component to the library manager tool. This system prompts for the XML (CBML) file that would be loaded into the system. When a new component is added, its name is displayed with the file name in the parenthesis in the component list. When a component is added, it is not a member of any groups and no services for its ports are defined. This needs to be defined by the user in order for the component to be used for building system. The delete option would simply delete the file from the list. It does not actually delete the file from the disk. Other operations are described in more detail in the following sections.

\subsubsection{Searching for Components}

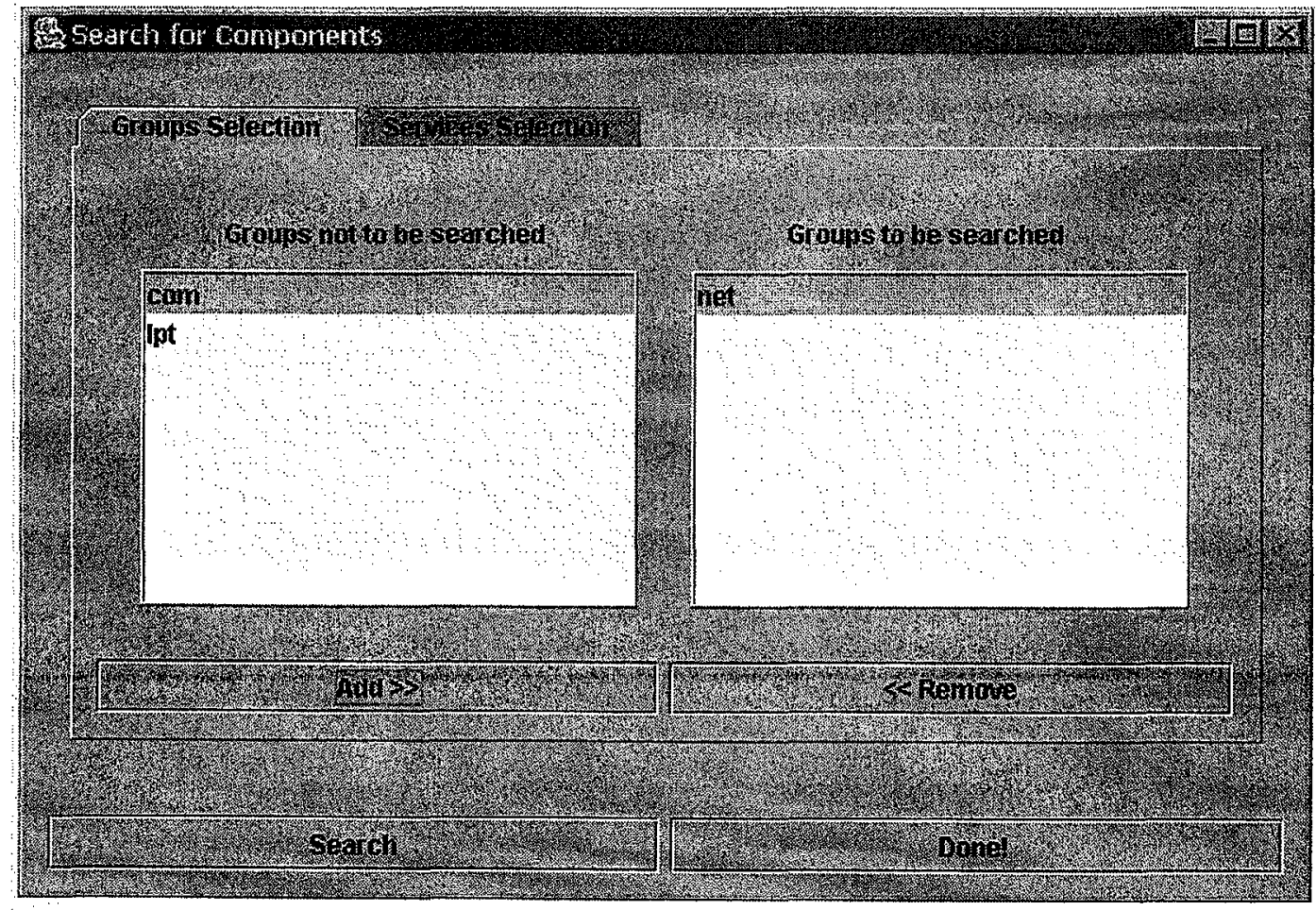

Figure 4-4: Search window for components with Groups selection pane active 


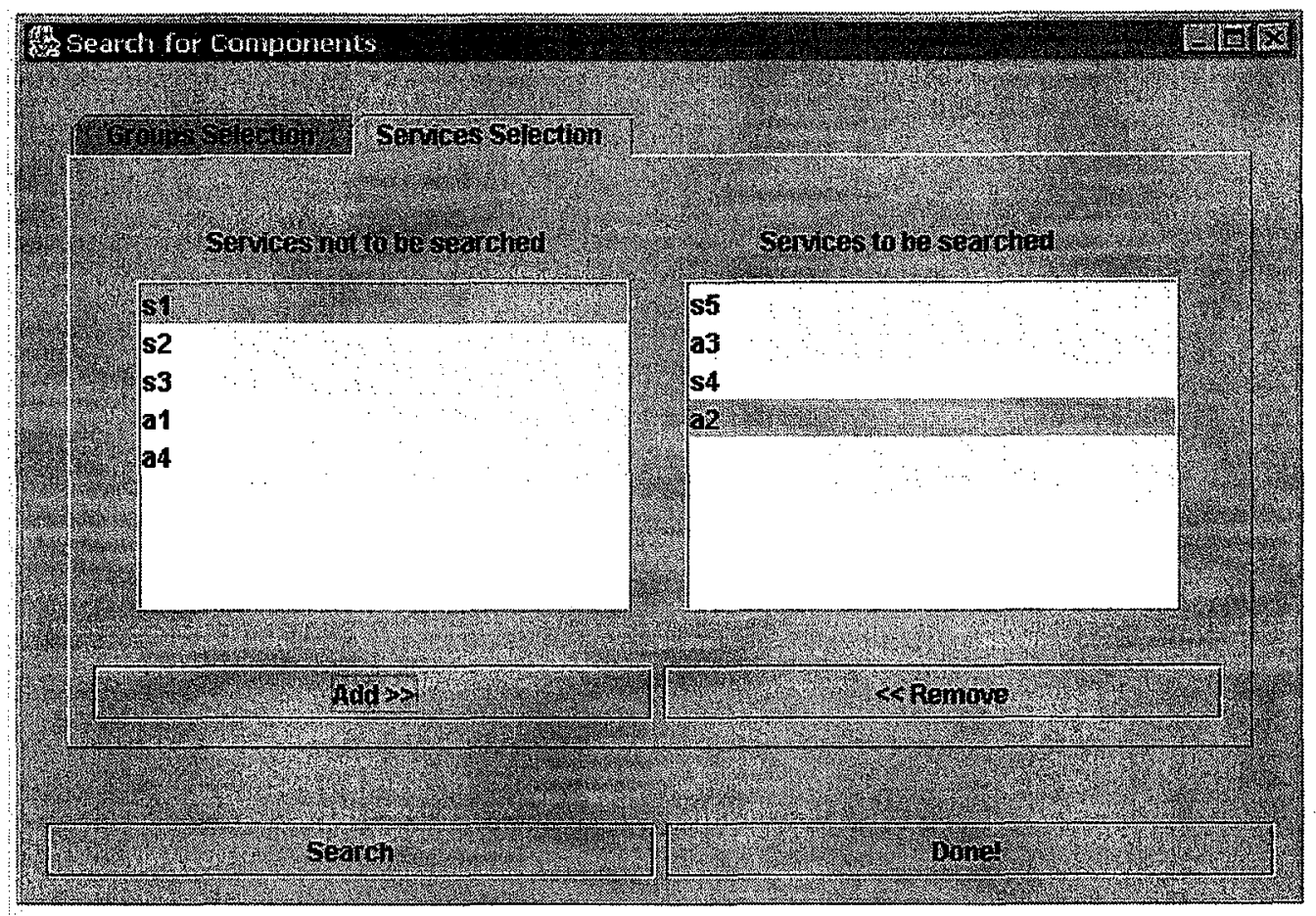

Figure 4-5: Search window for components with Services selection pane active

Search -20 results found!

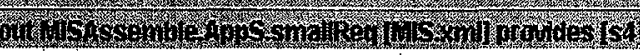

out MisAssemble.AppS.cachReq [nils.xm] provides [s5]

out AppComp.smallReq [AppComp.xml] provides [s4]

out AppComp.cachReq [AppComp.xml] provides [s5]

in Singlehod.p2 [SingleMod.xm]] requires [a2]

out Singlellod.p3 [SingleMod.xml] provides [a3]

out SimpleAppComp.smallReq [NewAppComp.xm] provides [s4]

out SimpleAppComp.cachReq [NewAppComp.xmi] prowides [s5]

in SimpleAppComp.S1.service2 [NewAppComp.xml] requires [a2]

out SimpleAppComp.S1.request 1 [NewAppComp.xm]] provides [a3]

out MISAssemble.AppS.smallReq [MIS.xm]] prouides [s4]

out MISAssemble.AppS.cachReq [MIS.xml] provides [s5]

out AppComp.smallReq [AppComp.xml] provides [s4]

out AppComp.cachReq [AppComp.xmn provides [s5]

in SinglelMod.p2 [SingleMod.xml] requires [a2]

out Singlehiod.ps [SingleMod.xml] provides [a3]

out SimpleAppComp.smallReq [NewAppComp.xml] provides [s4]

out SimpleAppComp.cachReq [NewAppComp.xml] prowides [s5]

in SimpleApnComp.S1. senice2 INowApnCamn.xmll reauires Ia21

Figure 4-6: Result list for search operation 
Searching for a component requires two kinds of information from the user. The first is the list of groups that need to be searched and the second is the list of services available on ports of components belonging to the list of groups supplied. These two pieces of information can be entered through the split pane window as shown in Figures 4-4 and 4-5. Figure 4-4 shows the window where groups to be searched are to be selected and Figure 4-5 shows the window where services that need to be searched are selected.

The result from search operation is displayed in a window similar to one shown in Figure 4-6. It contains the list of all components with their ports (and slots) where the required services were found.

\subsubsection{Group Settings for a Component}

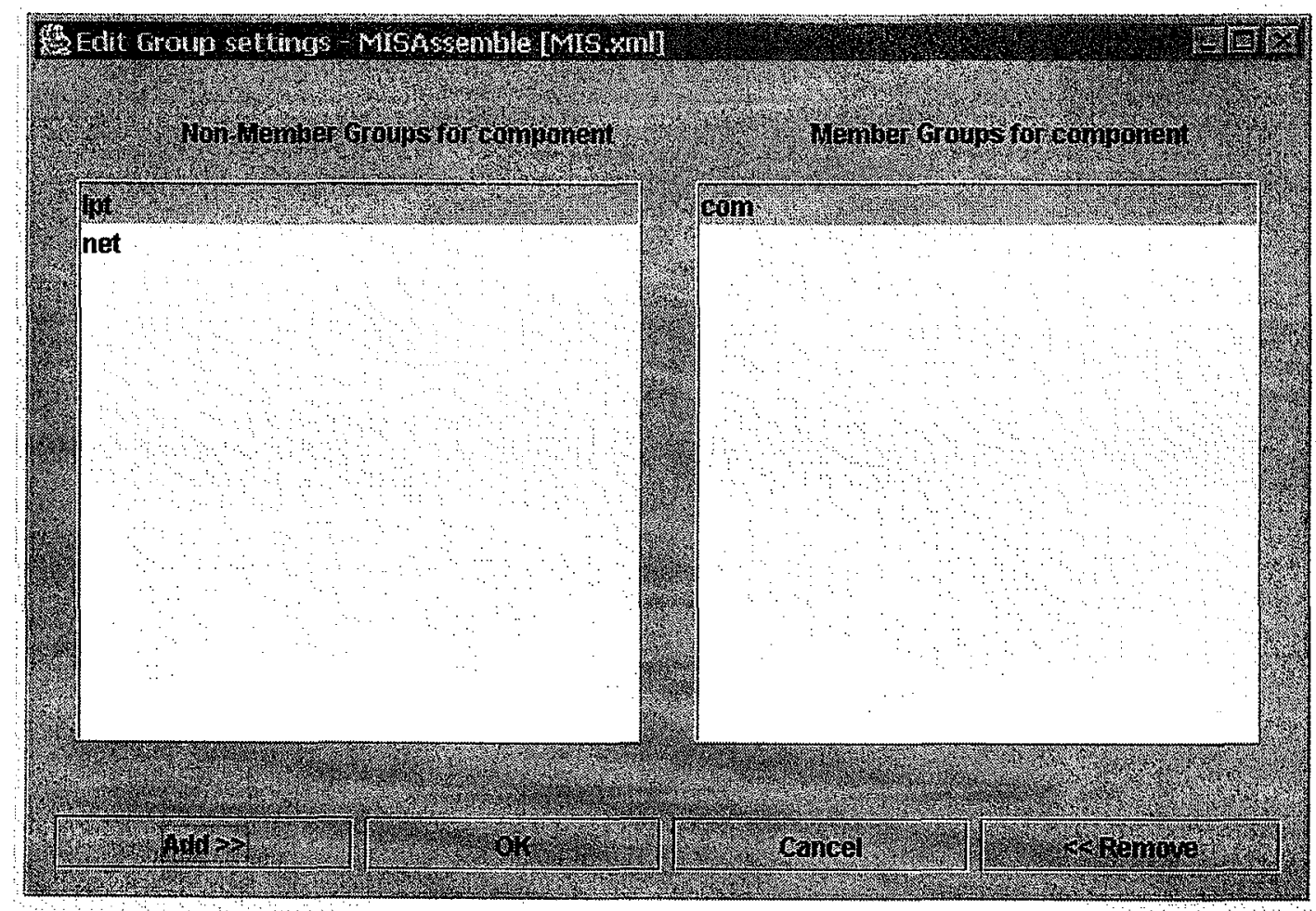

Figure 4-7: Groups setting for components 
Group setting involves altering the membership status of a component for various groups. This option lets the user decide which all groups should a component be a member of and which all groups should it not. A component needs to be selected from the component pane of main panel, shown in Figure 4-3, pressing the group settings button pops up a window, similar to one shown in Figure 4-7, with list of groups to which the component belongs to on the right side and list of groups to which the component belongs on the left side. The Add button moves the selected group on the left side to the right and while the remove button would move the selected group on the left to the right side. Pressing the Ok button would make these changes permanent and returns back to the component pane of the main window (the one shown in Figure 4-3), Pressing the cancel button would discard the changes and then return back to the component pane of the main window.

\subsubsection{Slot Settings for a Component}

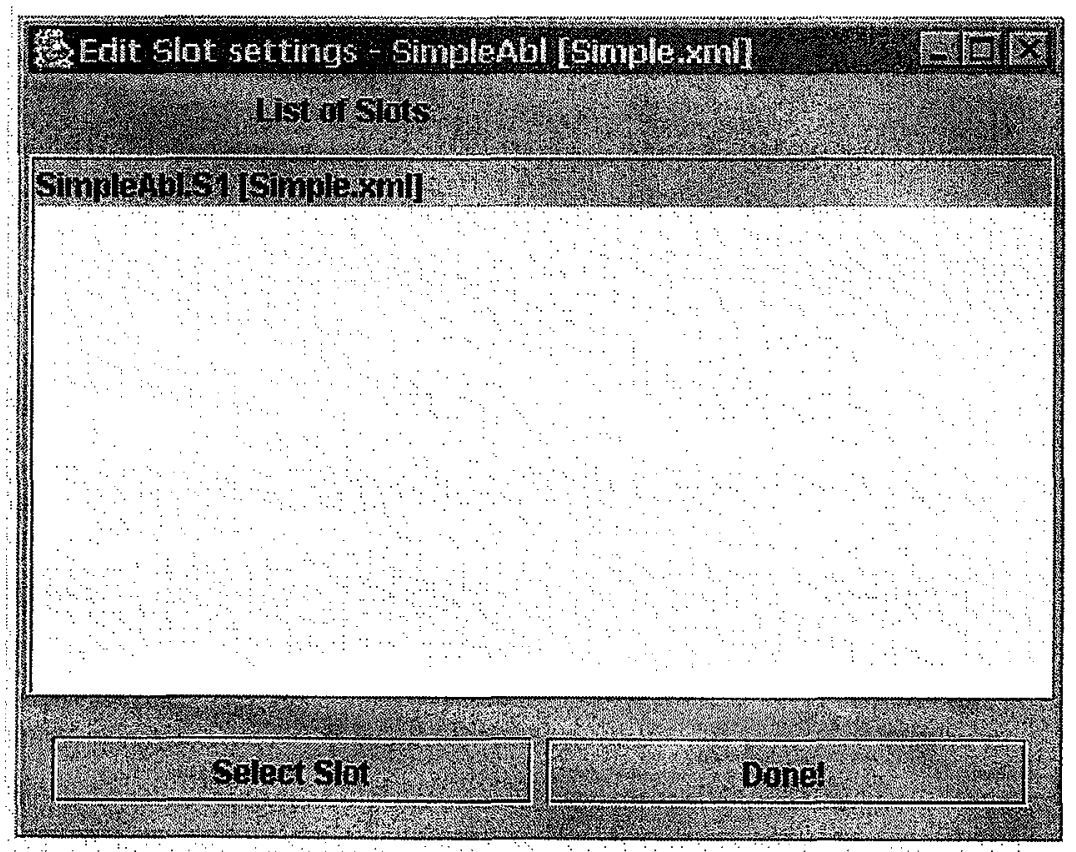




\section{Figure 4-8: List of slots for a component for slot settings}

Slot settings essentially alter the list of groups whose member's components can be bound to the slot. When a component is selected, a window similar to one shown in Figure 4-8 appears with list of slots present in the component.

A particular slot has to be selected which brings up the window similar to one shown in Figure 4-9 for editing the list of groups of components which can be bound to that slot. From the Add and Remove buttons, the status of each group can be changed from Non-Member to Member and vice versa. Pressing the Ok button will make these changes permanent. Finally, pressing the cancel button would discard changes.

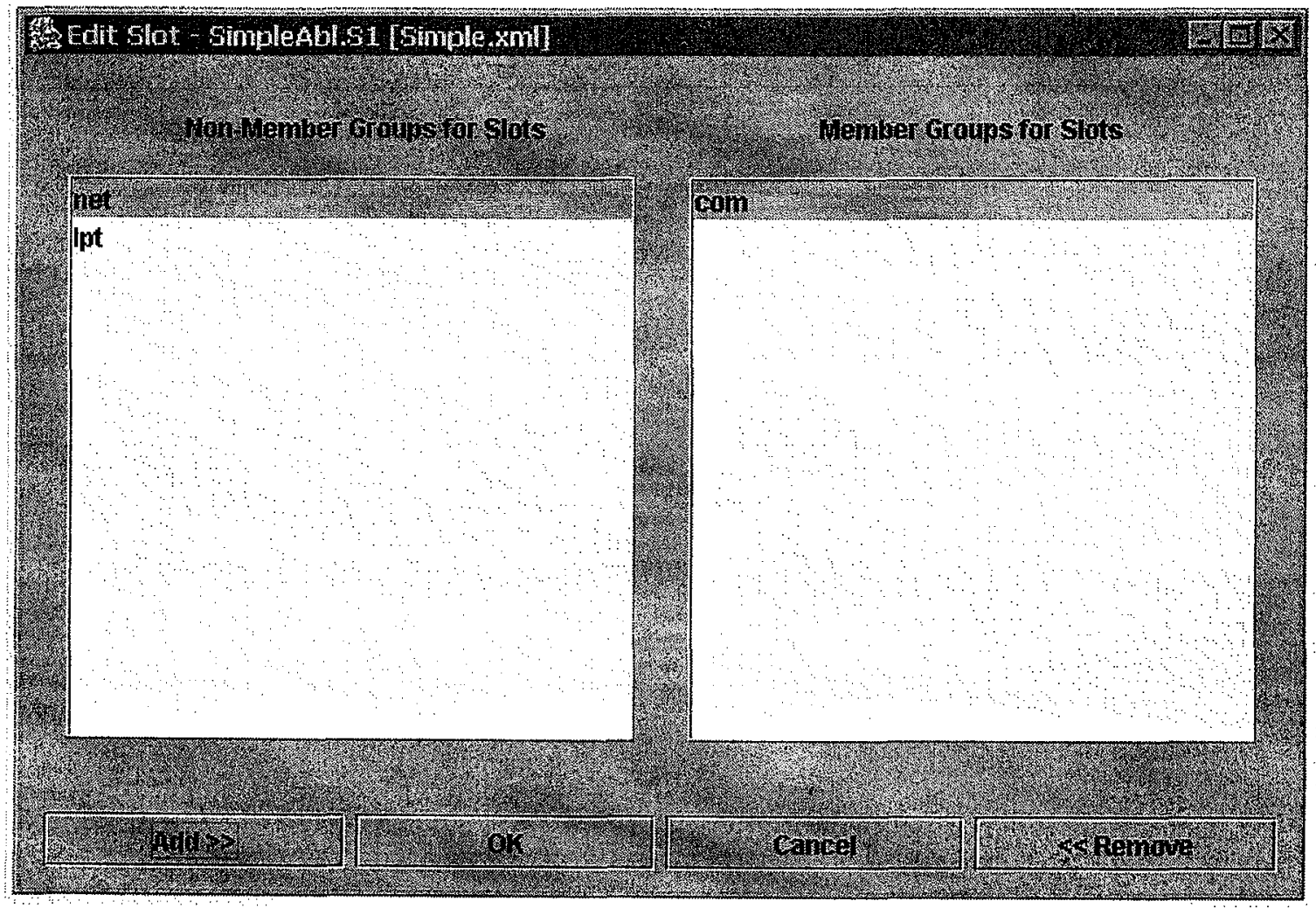

Figure 4-9: Member groups setting for component slots 


\subsubsection{Port Settings for a Component}

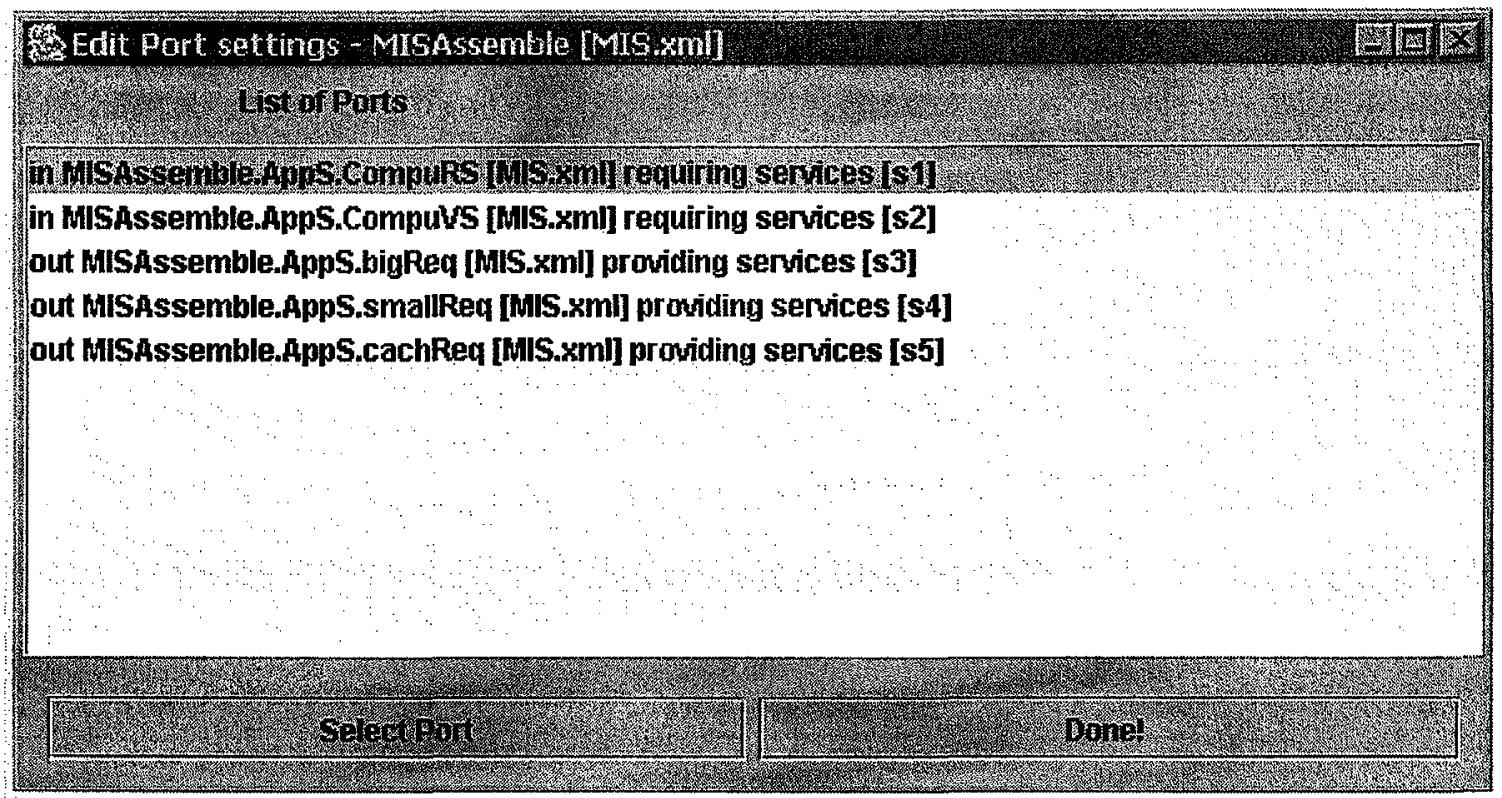

Figure 4-10: List of ports of interface and all slots of a component for port settings

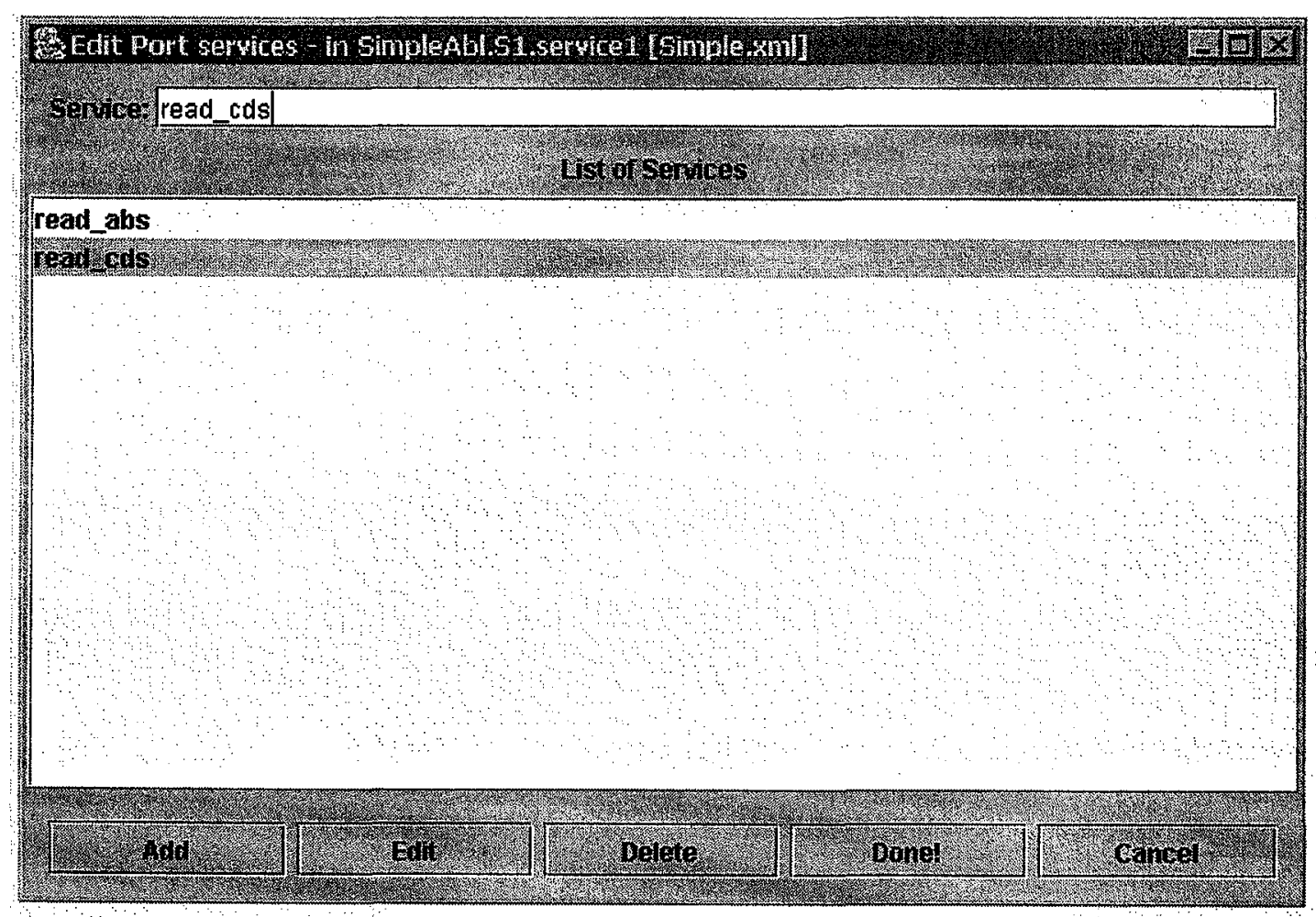

Figure 4-11: Editing services for a port 
Port settings essentially involve editing the list of services for a port. When a component is selected for its ports to be edited, a window similar to shown to Figure 410 is shown. All the ports of the component are displayed as shown in the figure below. The keyword "in" or "out" appears indicating whether the port is input port or output port. The name of the component, its slots hierarchy (if any) and the name of the port appear separated by a period followed by the XML file name. The list of services required/provided appears in the end. The port whose services need to be edited should be selected from this window.

When the port is selected, a window similar to one shown in Figure 4-11 comes up. The controls on this window enable the user to enter edit or delete services. There is a text field on top where new services can be entered. Before finishing, user clicks on the "Done!" button to finalize the change. Clicking on the "Cancel" button discards all the changes made.

\subsection{Building Systems from Components}

Finally having all those components in place, we would like to build systems out of them. There are two approaches to building systems from components. The first one is done by manually selecting all the components for the slots from the list of permissible choices. The second one involves automatically enumerating all possible combinations. These two approaches can used in combination i.e. some of the slots can be filled manually while others can be enumerated. 


\subsubsection{Tree View of Component Assembly}

In order to use the tool to build systems, we need to understand another view of component, namely the tree view of component. This is necessary to understand the core assembly algorithm used for automatic enumeration of components as well as use the tool to build systems. The tool uses tree notation as its display

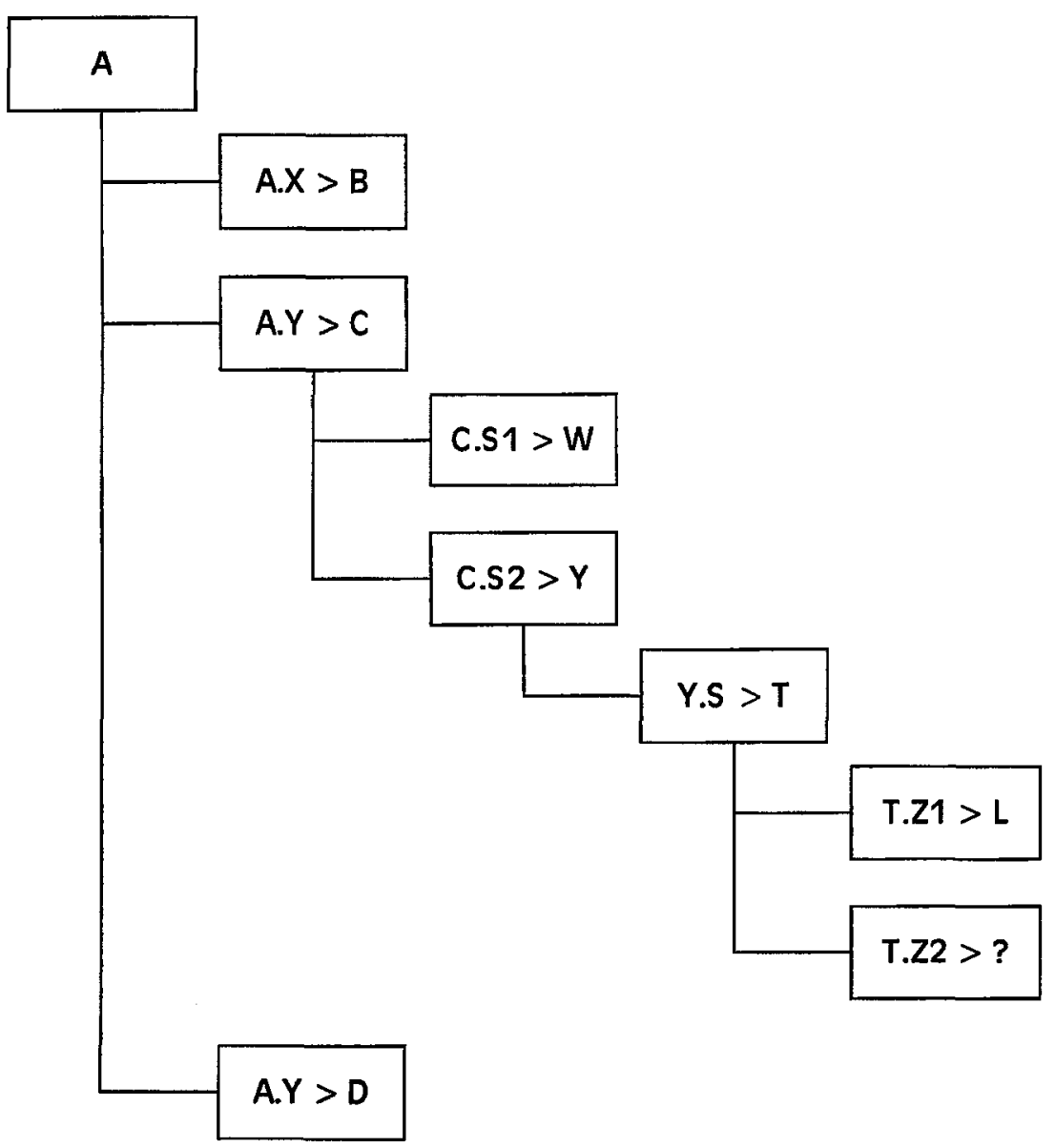

Figure 4-12: Example of a tree where a binding decision needs to be taken

CBML views of a component assembly as components having slots where other components have been bound. Alternatively we can view a component assembly as a tree 
with the main component being the root node and all the components having slots where other components can be viewed as nodes having child nodes.

Figure 4-12 shows an example of such an assembly. Here the main component is A which is represented as the root node. It has three slots $\mathrm{X}, \mathrm{Y}$ and $\mathrm{Z}$ to component $\mathrm{B}, \mathrm{C}$ and $\mathrm{D}$ respectively. These are shown as three different child nodes to the root node. The component $\mathrm{C}$ has two slots $\mathrm{S} 1$ and $\mathrm{S} 2$ bounded to components $\mathrm{W}$ and $\mathrm{Y}$ shown as two child nodes to the node C.S2. The component $\mathrm{Y}$ has a slot $\mathrm{S}$ bound by component $\mathrm{T}$ as shown in the figure. Finally $\mathrm{T}$ has two slots $\mathrm{Z1}$ and $\mathrm{Z2}$. $\mathrm{Z1}$ is bound to component $\mathrm{L}$ however Z2 is not bound to any component and hence "?" appears at the end. Here the symbol ">" represents slot bound to a particular component.

\subsubsection{Building Systems manually using the Tool}

Building systems involve selecting a main component (which acts as the template as explained in section 3.3.1) and filling its slots with components. When a component is selected for building, all the slots of this component appear as a nodes of a tree with the component as the root node similar to Figure 4-13 and Figure 4-15. There are four buttons that appear below the tree view of the main component. These buttons are "Build", "Bind" (or "Unbind" depending on the current state), "Auto-Build" and finally, "Done!" .

To bind a slot, the user needs to select a node corresponding to an empty slot in the component tree. When an empty slot is selected, the second button displays the label "Bind" as shown in Figure 4-13. Clicking on it brings up a window similar to one 
shown in Figure 4-14 with all the possible alternative components for this slot and the user is expected to select one. Selecting one of them and clicking the "Bind Component" button would fill the slots with that component while clicking the "Cancel" button would close this window and bring back control to window shown in Figure 4-13. When a component is bound to an empty slot, all the slots of this component appear as child node, to the node which corresponds to the slot in which the new component is bound.

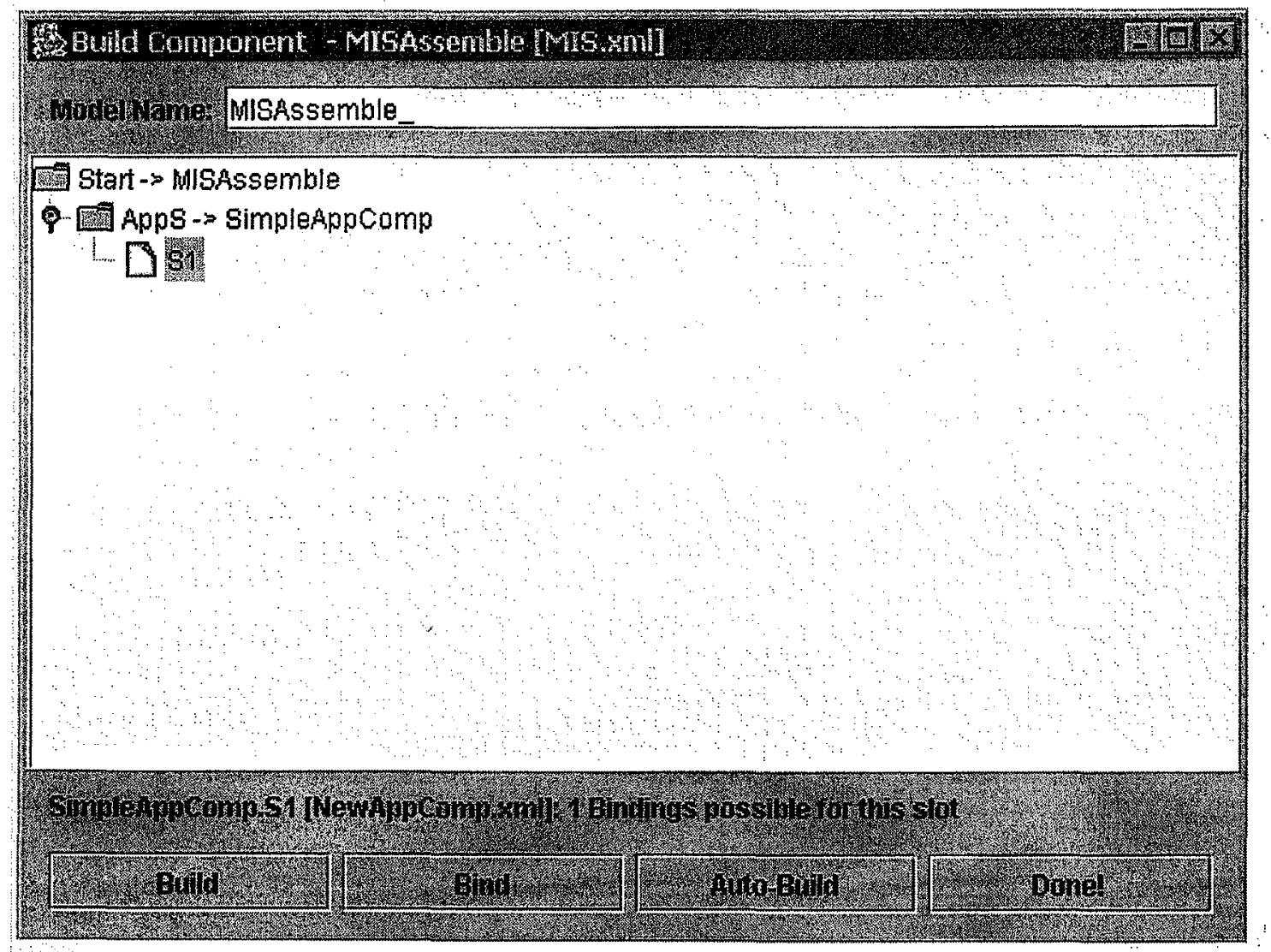

Figure 4-13: Build tree for a component with an empty slot selected for binding 


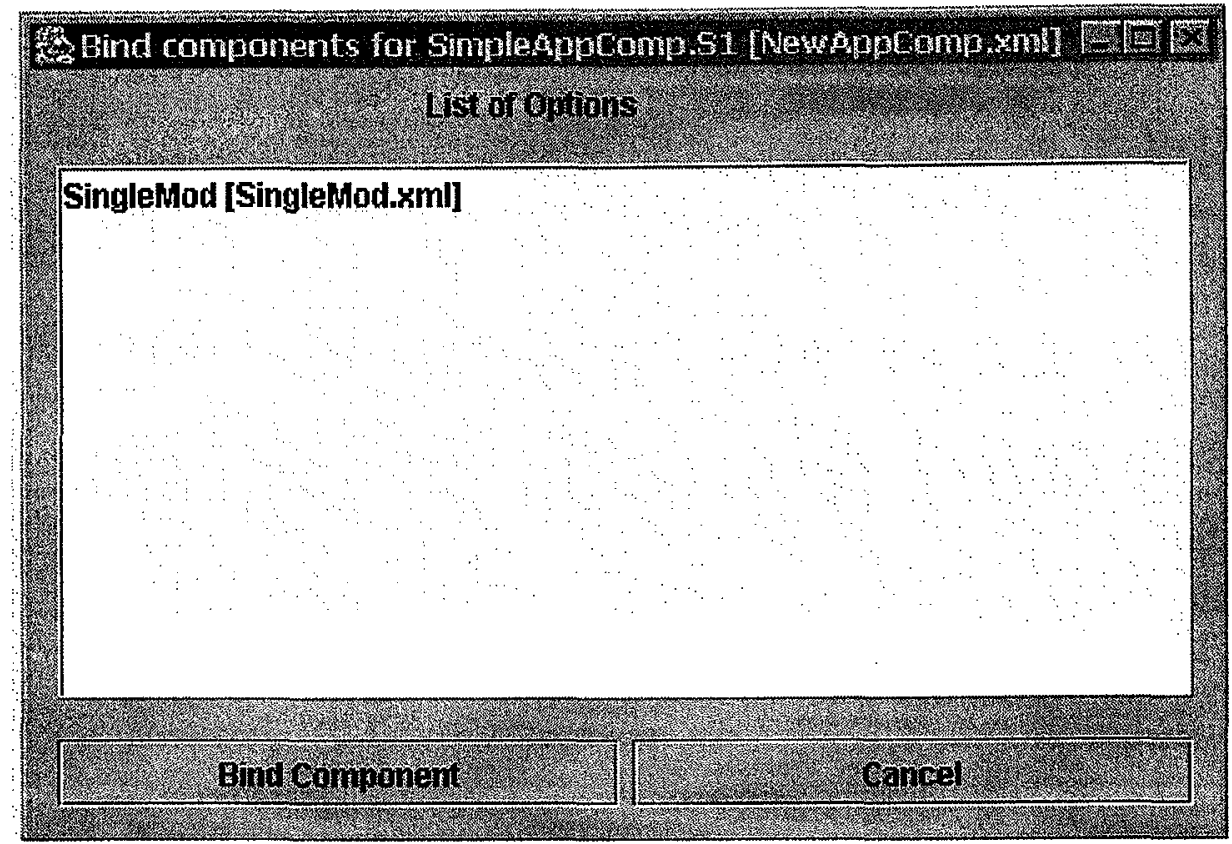

Figure 4-14: Select a component for binding into a slot

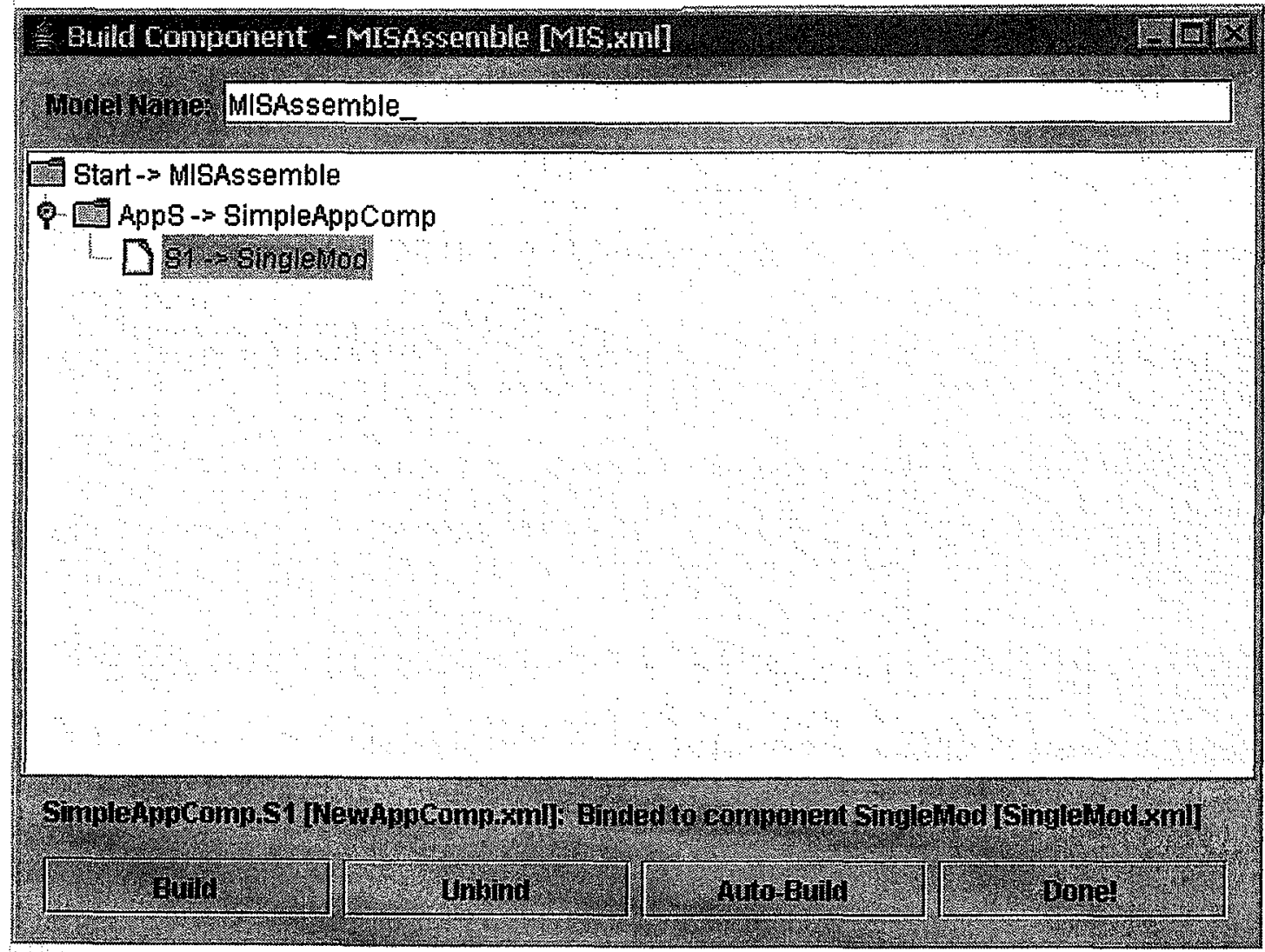

Figure 4-15: Build tree for a component with a filled slot selected for unbinding 
To unbind a slot, the user needs to select a node corresponding to a filled slot. This will change the label of the second button to "Unbind" as shown in Figure 4-15. Clicking on the "Unbind" button will unbind the slot. It should be noted that that when the node corresponding to a filled slot is unbounded, all the child nodes are removed.

The process of filling (and emptying) slots with components can be repeated any number of times to build a component tree. This approach can be used to build either a partial system or a complete system. It is often useful to fill some slots and leave the rest empty. The remaining ones can be filled by automatic enumeration if so desired.

Finally the system can be saved to the disk by using the "Build" option. This will create a new component with all the filled slots merged completely by their components. Also the text field on top of the tree can be used to edit the name of this newly built system.

\subsubsection{Automatic Assembly of System}

It is useful in many circumstances to enumerate all the possible combinations and the tool provides this option through the "Auto-Build" button. Pressing this option will enumerate all the permissible combinations possible. The Algorithm used for automatic enumeration of all alternatives is given below. It is assumed that we have partially built tree. Automatic assembly of single components can be treated as a special case of a partially 
evaluated tree having the component as the root node and all its empty slots as its child nodes.

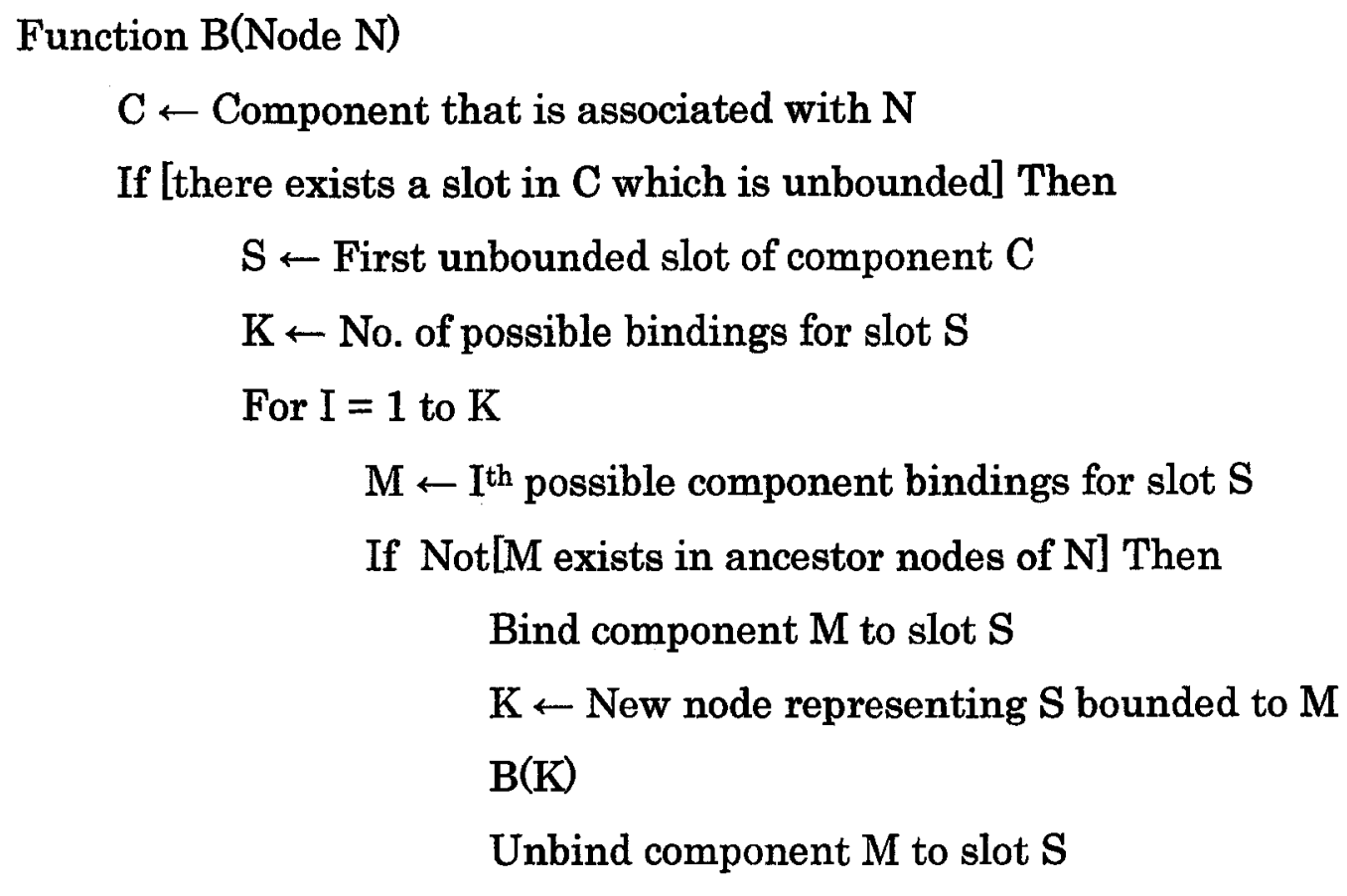

End If

End For

Else

If [ $\mathrm{N}$ is root] Then

Cache the solution with $\mathrm{N}$ as root node and return Else

$\mathrm{B}($ Parent of N)

End If

End If

Return

In the above algorithm, the function $\mathrm{B}(\mathrm{)})$ is the building function which takes an input parameter N. As stated previously, we already have a partially built tree (built by manual 
user selection) whose root node is $\mathrm{N}_{0}$. Then the build function is invoked on this node by calling $\mathrm{B}\left(\mathrm{N}_{0}\right)$ and all the alternatives are enumerated and cached.

After the models are generated, a file dialog prompts for the name of the file to which the models will be stored. The models are stored as components into these files except the names of their individual files will also have a number attached to differentiate one model form another. For example, suppose there are four different alternatives and user selected the name of the file as "gen.xml" then these models would be saved as "gen_1.xml", "gen_2.xml", "gen_3.xml", and "gen_4.xml".

\subsection{Conclusion}

The tool described in this chapter can be used to store and manipulate CBML components and it can be used for building systems out of these components.

It provides two approaches to building systems - a manual approach where the user selects all the components and automatic approach where all the possible alternatives are enumerated. Both the approaches can be used in combination with each other. In practice the best approach is to fill some slots manually where a particular component is suitable and automatically fill the sl'ots where there are multiple number of choices available. 


\section{Chapter 5: A Case Study: A Distributed Telephone}

\section{$\underline{\text { Switch }}$}

This chapter applies the ideas described in this thesis to a practical problem of designing parts of a distributed telephone switch. It starts with the description of the switch and a set of alternatives, and explains the methodology of building a performance model for all of the alternatives and evaluating each one of them.

\subsection{The Distributed Telephone Switch (DTS)}

One of the areas where performance issues are important is telecommunications switching systems. The architecture of digital voice switches that are used in the industry today are the result of over 40 years of evolution. The evolution of these systems has been dramatic. For example in twenty years, the 5ESS has been scaled from under 10,000 line terminations (individual telephone lines) to over 200,000 line terminations, and from 100,000 call attempts per hour to over 2,500,000 call attempts per hour. The details of evolution of Lucent's 5ESS voice switch architecture are described in [24] with emphasis on the following performance aspects: continual performance improvement, the tradeoffs between performance and other properties, call flows and delays, overload control, and software resource engineering. 


\subsubsection{Need for Performance Engineering}

Provisioning of residential telephone services is highly regulated. Governmental agencies like Canadian Radio and Telecommunications Commission (CRTC) for Canada set standards for service providers, e.g. [7], [29], [30]. These standards can be from the end customer's point of view such as: "a user must receive dial tone within 0.5 seconds, $98 \%$ of the time" or "post dialing delay should not exceed 0.4 seconds for $99.9 \%$ of call attempts at an average busy season busy hour load level2 (Post dialing delay is defined as "the time interval between the end of dialing and the occurrence of an audible network response, other than switching noise, to the caller.). Alternatively there may be interconnection standards like strict call processing times for SS7 TCAP and ISUP layers.

One reason cited in [31] for the slow rate of adoption of Voice over Packet (VoP) solutions is that performance issues have not been paid enough attentions. This may in part be due to the lack of use of SPE in the development of data switching equipment, especially at the requirements stage.

\subsubsection{Architecture of a DTS}

Voice switches are organized into a two level hierarchy. At the lowest level, class 5 switches have subscriber line interfaces and network trunk interface. At the highest level, class 4 switches have trunk interfaces to interconnect both class 5 and other class 4 switches. Inter-switch communication is almost exclusively performed out of band via the SS7 protocol. Also attached to the network, via SS7 protocols are databases. These databases are the enablers for a number of modern telephone features, from "800" 
service, to local number portability, to call forwarding. More information about architecture of a distributed switch can be found in [32]. Figure 5-1 provides an abstract view of the current network.

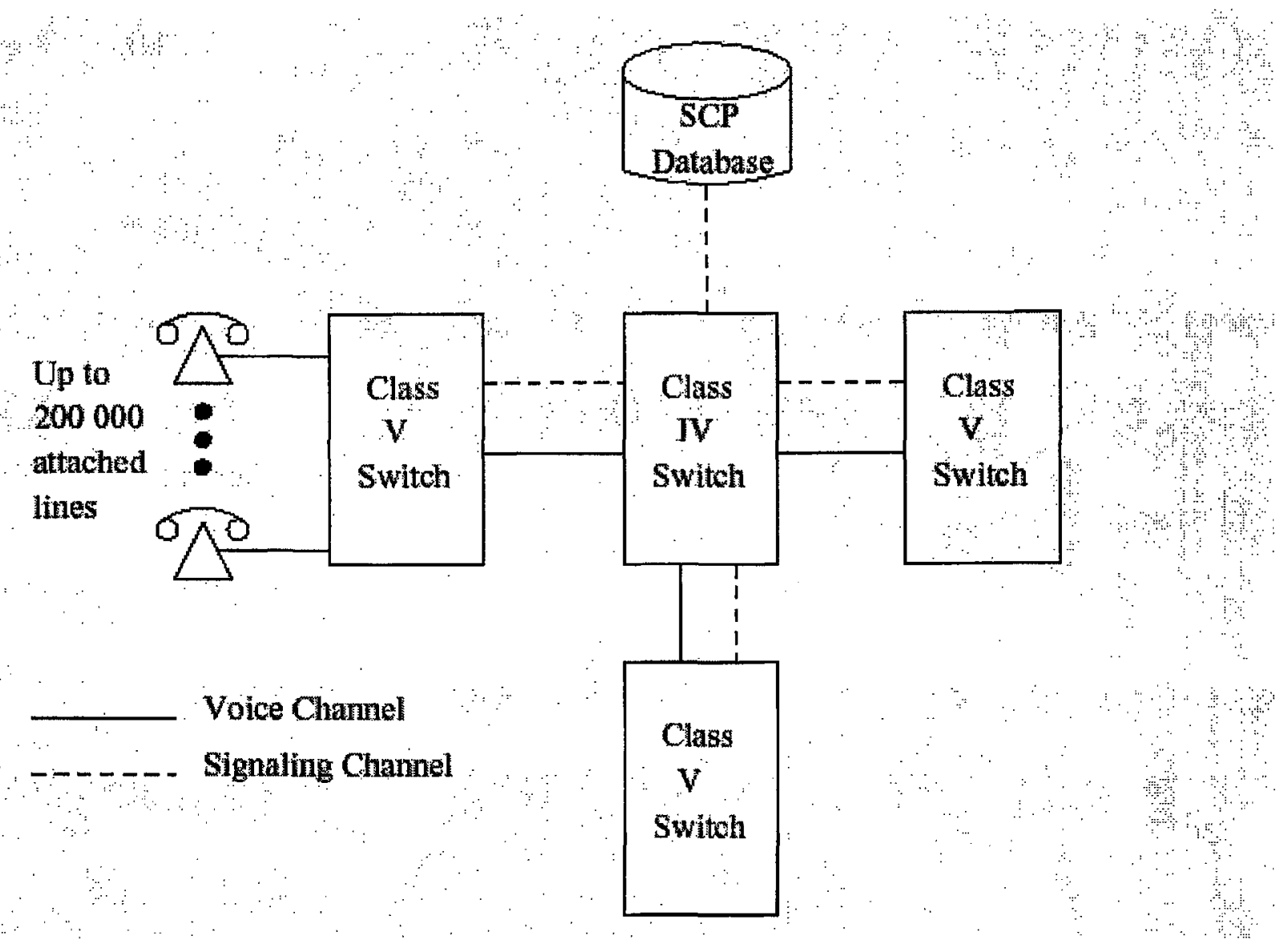

Figure 5-1: Abstract view of current network

The Class 4 switch employs a call connection agent, which consists of a number of servers connected by an internal network, that perform call processing, interfacing to the trunking gateways and interfacing to external voice switches. The IETF/ITU Protocol H.248 [13] is used for communications between the call connection agents and the 
trunking gateways. To an external voice switch, the combination of the call connection agent and trunking gateway appears as a class 4 switch. The Call connection contains the following elements:

- Line Information Database: The database that contains all of the information required about the attached gateways in order to make routing decisions.

- Call Processing Server: The server that does the bulk of the call processing work making call routing decisions and coordinating connectivity.

- SS7 Interface Processor: The server that is the call connection agent's interface to the traditional telephone network. It converts SS7 protocol interactions into a set of internal call control messages.

- H248 Interface Processors: The server that is the call connection agent's interface to the VoP network. It converts H.248 protocol interactions into a set of internal call control messages.

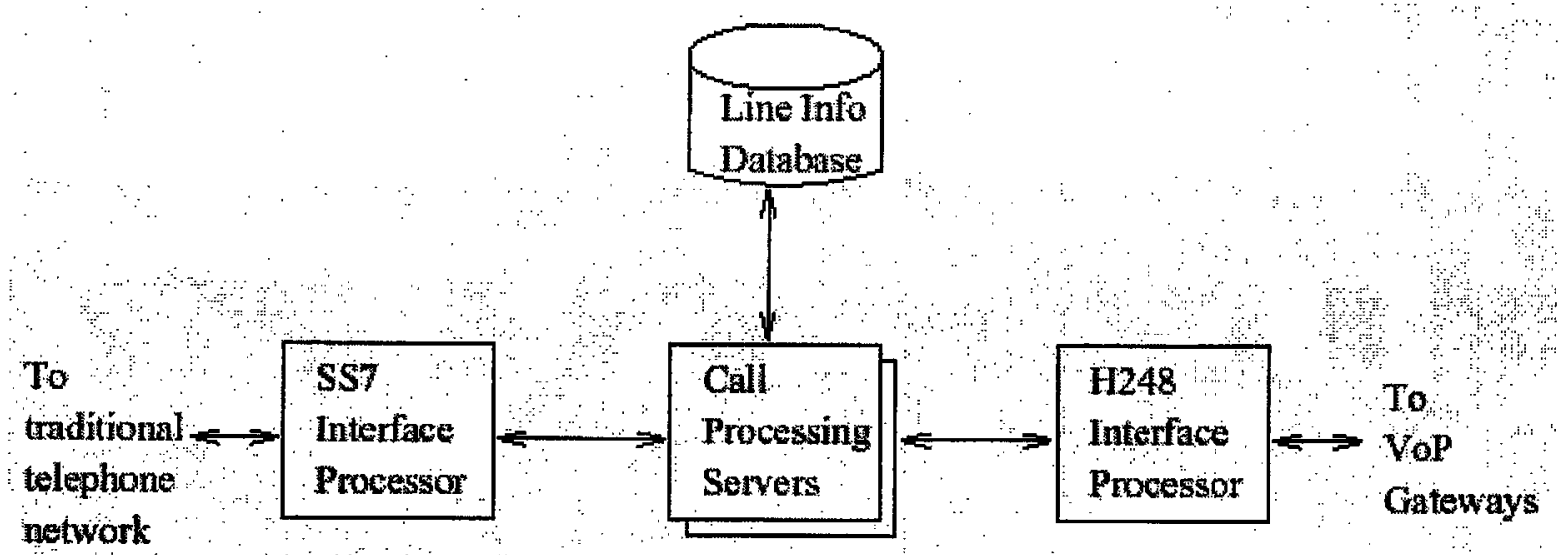

Figure 5-2: Servers of Call Connection Agent connected by internal network 
Figure 5-2 gives a graphical representation of the servers in the call connection agent. The trunking gateway switch, as shown in Figures 5-3, contains processing modules (cards) that are interconnected by an intra-switch network. These are:

- H248 Call Processing Card module that terminates H.248 messages and converts them into internal switched services connection requests.

- Control Card module that provides the overall control and connection database for the switch.

- SVC Call Processing Card module that terminates the switched services connection requests and provides an interface to the ATM signaling network. All connections on the switch are created via ATM switched virtual circuit (SVC) signaling methods.

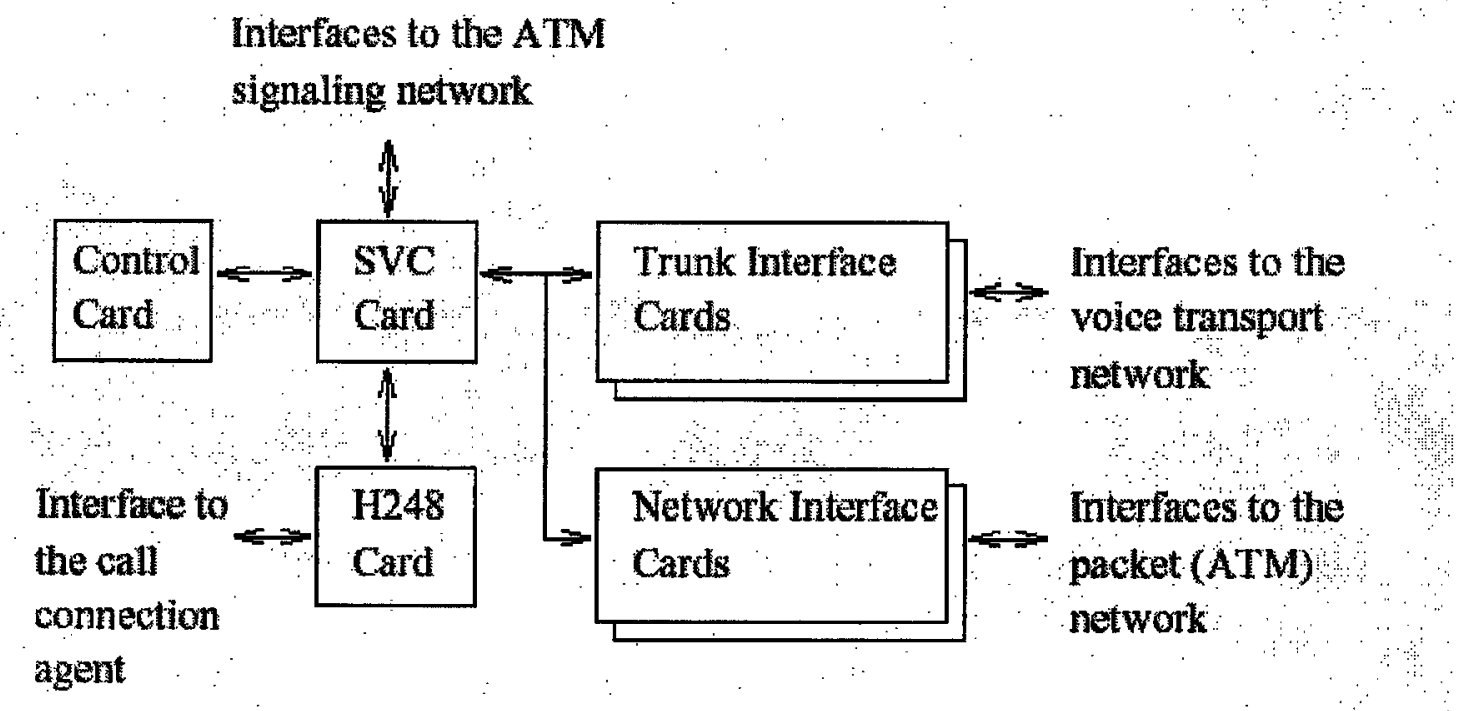

Figure 5-3: Servers of Call Connection Agent connected by internal network 
The core packet switch is built using the SVC Call Processing, Control, and Network Interface cards from the gateway, and runs similar software.

\subsection{Modeling the Distributed Telephone Switch (DTS)}

In this study, we use a simplified model of a VoP distributed class 4 Switch adapted from [32]. The design of the DTS starts with building a template model for the system and identifying and formulating constraints for the system. It then involves selecting the components and classifying them into groups based on their functionality and labeling services on their ports. Subsequently we use our tool to create a library of components including the template and generate all the possible alternatives. Finally constraints are mathematically formulated and all the alternatives are ranked after applying the constraints.

\subsubsection{Template Model of a Telephone Switch}

Figure 5-4 shows the template of the system with slots for different components. UserTask represents the reference task with of 1000 active callers, with a think time of $0.5 \mathrm{sec}$. The template consists of six slots of which two are gateways, three are switches and one is a call connection agent. Components with different kinds of concurrency will be described for these slots below. There are three processors shown: MainP, SwitchP, and GatewayP. In addition, there is an generating infinite processor, UserP for the load generating task. 


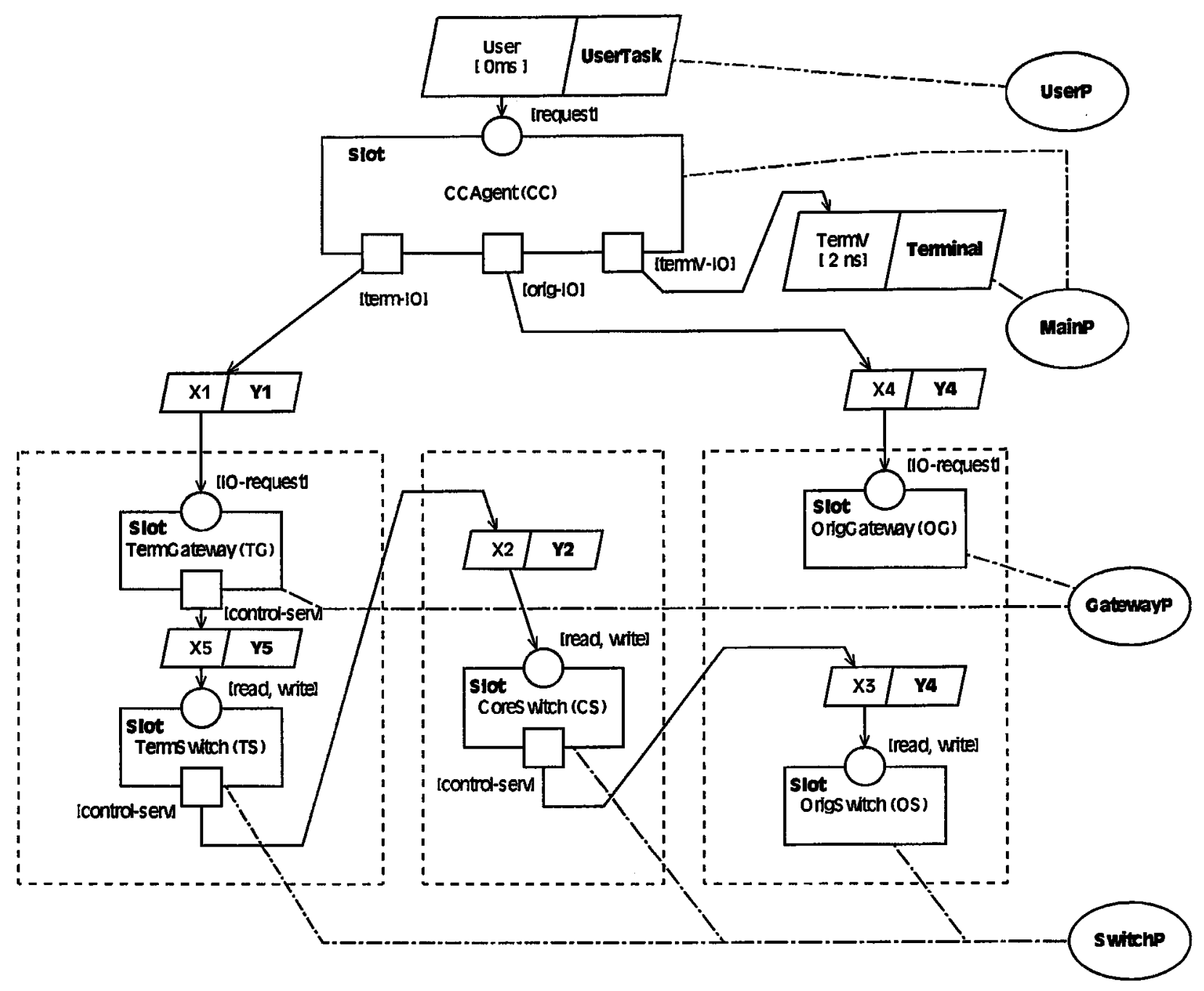

Figure 5-4: Template for the Distributed Telecom Switch

\subsubsection{Constraining the System}

After generating the systems, we would like to formulate the constraints that would enable us to screen out the undesired alternatives and rank the desired ones. Hard constraints are formulated first. They are based on throughput for the user, response time for the system and utilization for processors. They are shown in Table 5-1. 


\begin{tabular}{l}
\hline \multicolumn{1}{c}{ Hard Constraints } \\
\hline (1) $\mathrm{F}_{\text {UserTask }} \geq 500$ request $/ \mathrm{sec}$ \\
(2) $\mathrm{R}_{\text {Input }} \leq 2 \mathrm{sec}$ \\
(3) $\mathrm{U}_{\text {SwitchP }} \leq 80 \%$ \\
\hline
\end{tabular}

Table 5-1: Hard constraints for Distributed Telephone Switch example

The throughput of UserTask should be at least 500/sec. This is formulated as the first hard constraint. Similarly the minimum response time for the system should be capped and here we chose that value to be $2 \mathrm{sec}$. This is formulated as the second hard constraint. Finally the utilization of the SwitchP processor should be less than $80 \%$. This is formulated as the third hard constraint.

Similarly we formulate soft constraints for the system. The soft constraints for the system are shown in Table 5-2 with their penalties shown in braces.

\begin{tabular}{ll}
\hline \multicolumn{2}{c}{ Soft Constraints } \\
\hline (1) $\mathrm{R}_{\text {TermGateway }}+30 \times \mathrm{R}_{\text {OrigGateway }} \leq 300 \mathrm{~ns}$ & $\{4\}$ \\
(2) $\mathrm{U}_{\text {GatewayP }}+8 \times \mathrm{U}_{\text {MainP }} \leq 12 \%$ & $\{8\}$ \\
(3) $\mathrm{R}_{\text {Coresw }} \leq 30 \mathrm{~ms}$ & $\{1\}$ \\
\hline
\end{tabular}

Table 5-2: Soft constraints for Distributed Telephone Switch example with penalties shown in braces \{\}

The first constraint is chosen as a weighted balance between response times for terminal gateway and originating gateway. The second constraint is chosen as a weighted balance between utilizations of SwitchP processor and MainP processor. Finally the third constraint is put as a limit to the response time of core switch. 


\subsubsection{Components of a Telephone Switch}

There are components that need to be put into the slots of the main template. The components here are grouped according to their functionality and the slot they fit in. The slots in the template of Figure 5-4 have been abbreviated and are shown in Table 5-3.

\begin{tabular}{l|c}
\hline \multicolumn{1}{c|}{ Slot } & Abbreviation \\
\hline CCAgent & CC \\
TermGateway & TG \\
TermSwitch & TS \\
CoreSwitch & CS \\
OrigGateway & OG \\
OrigSwitch & OS \\
\hline
\end{tabular}

Table 5-3: Abbreviation used for slot of template

The CBML code for the components is described in Appendix B and is summarized in Table 5-4. Diagrams for components are given here. There are four different kinds of gateway components. They are shown in Figure 5-5, Figure 5-6, Figure 5-7 and Figure 58. Of these, two of them are input only gateway and the other two are input/output gateways. The Main the difference between the gateways are that the operations for the gateway protocol GwH248 and CACO agent are bundled together for the first and the third component in Figure 5-5 and Figure 5-7, and separated in Figure 5-6 and Figure 58. 


\begin{tabular}{l|c|l}
\hline Component & Slots & \multicolumn{1}{c}{ Description } \\
\hline C1 & CC & A simple call connection agent \\
C2 & CC & A concurrent call connection agent \\
G1 & OG & A simple gateway with one input port \\
G2 & TG & A concurrent gateway with one input port \\
G3 & OG & A simple gateway with one input \& one output port \\
G4 & TG & A concurrent gateway with one input \& one output port \\
S1 & OS & A simple switch with one input port \\
S2 & TS, CS & A concurrent switch with one input port \\
S3 & OS & A simple switch with one input \& one output port \\
S4 & TS,CS & A concurrent switch with one input \& one output port \\
\hline
\end{tabular}

Table 5-4: Abbreviation used for slot of template

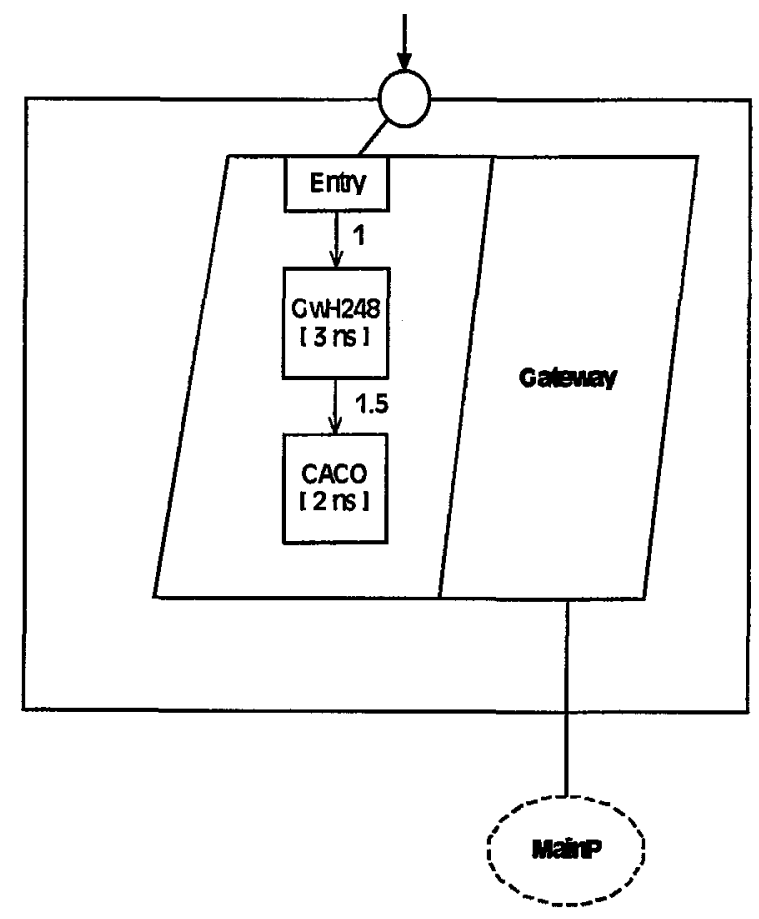

Figure 5-5: First Gateway component - G1 


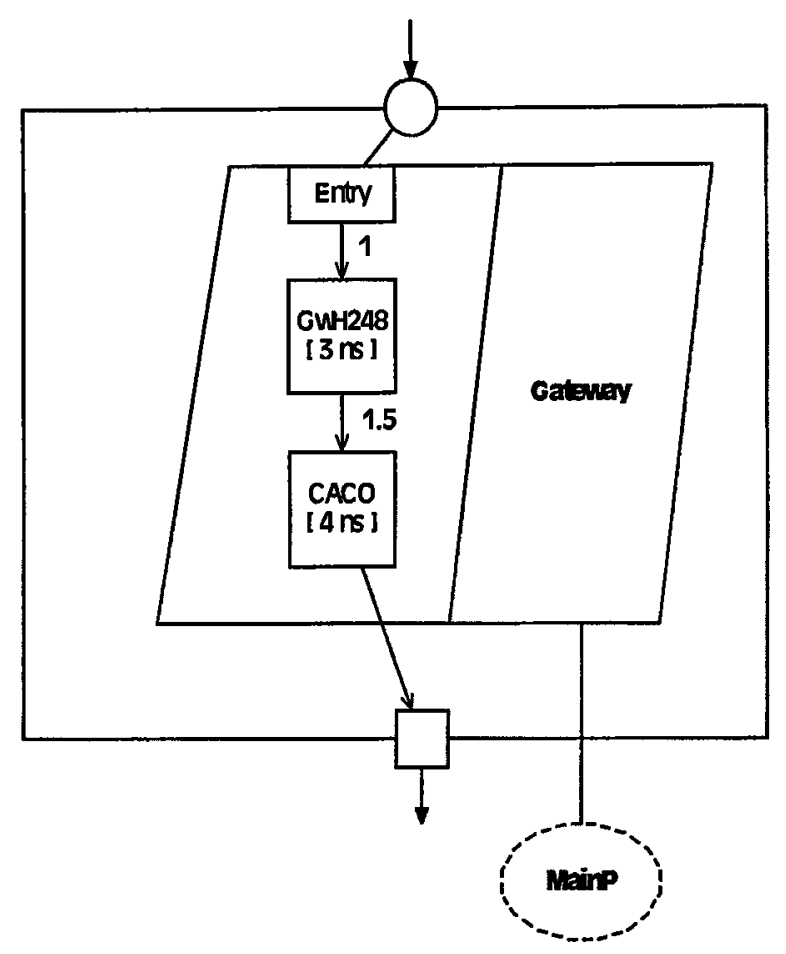

Figure 5-6: Second Gateway component - G2

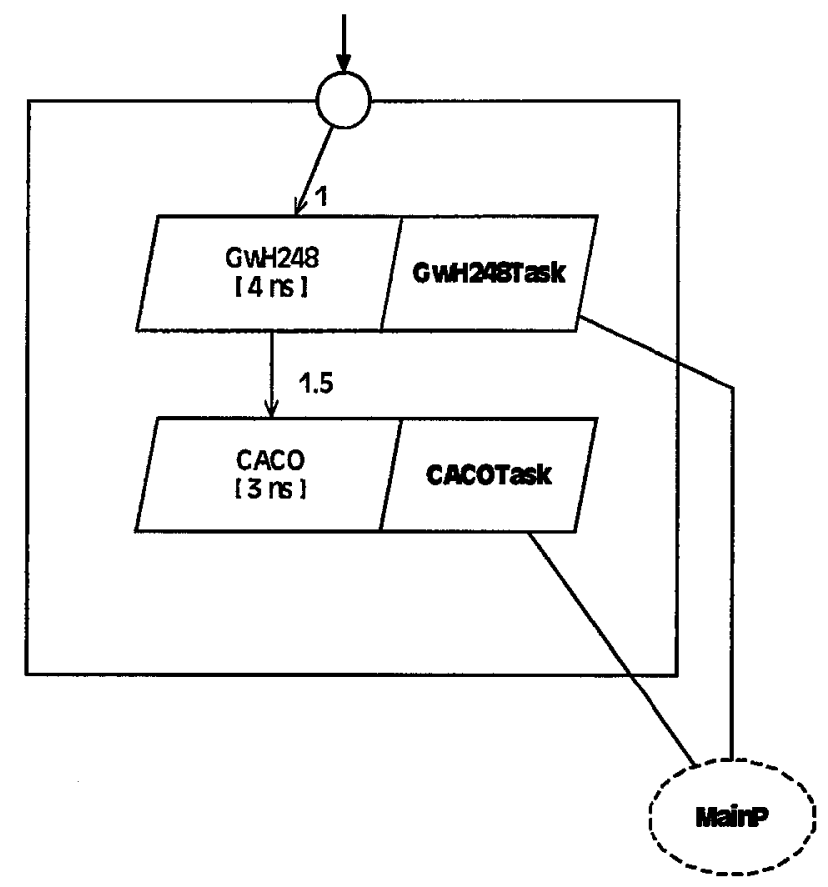

Figure 5-7: Third Gateway component - G3 


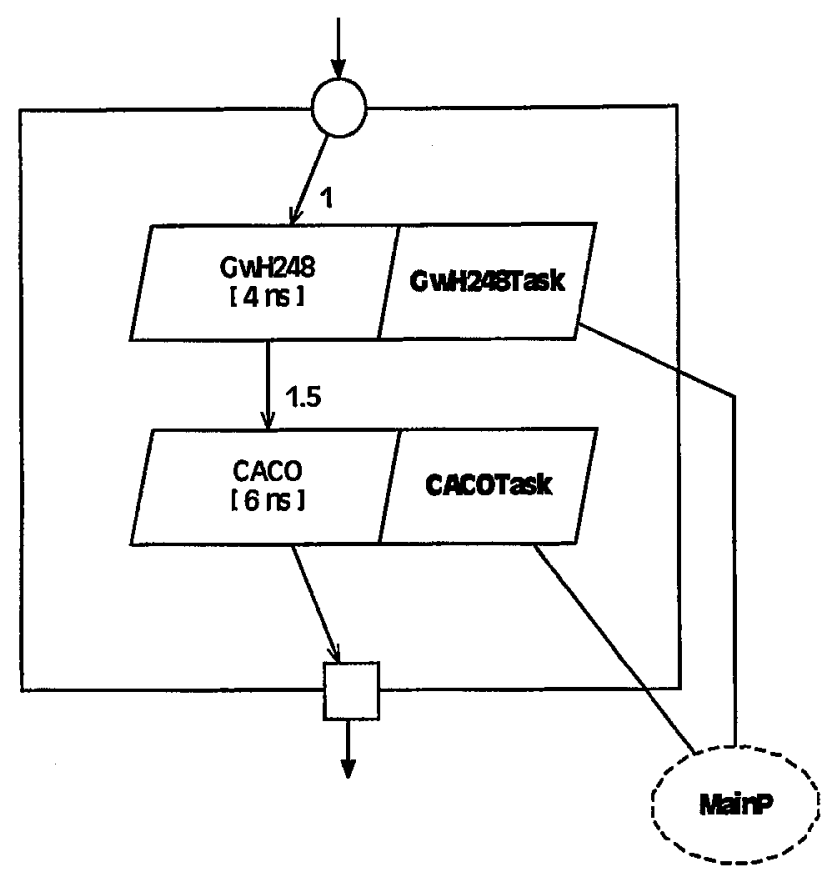

Figure 5-8: Fourth Gateway component - G4

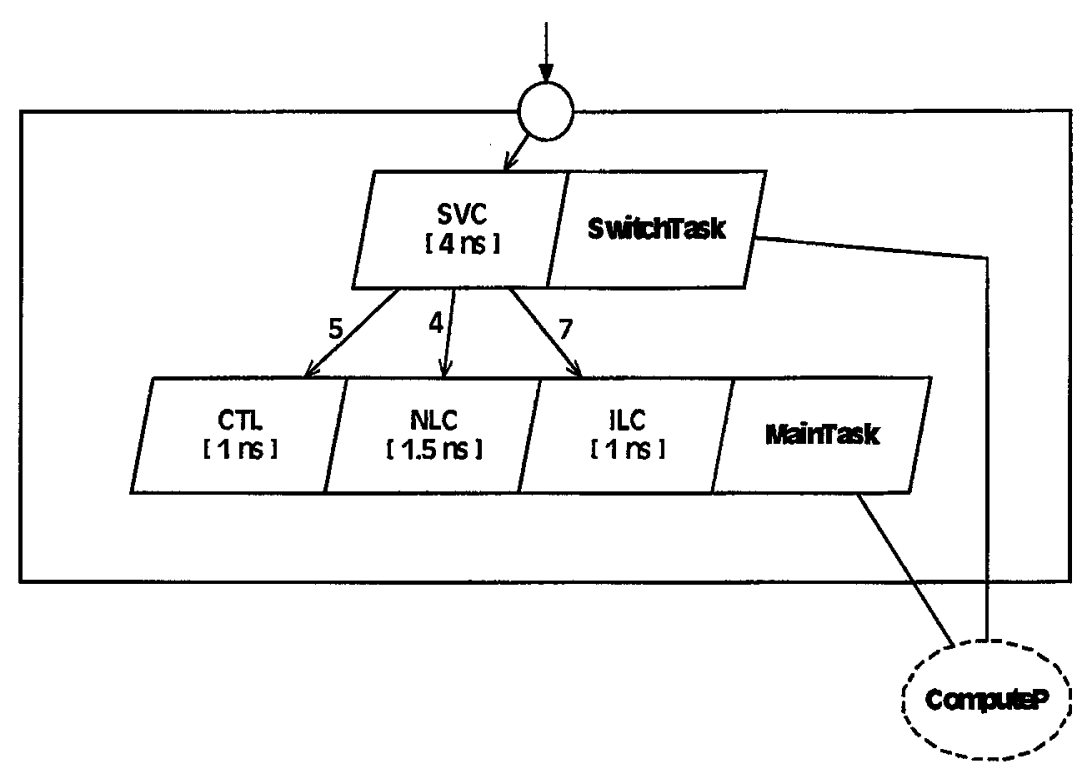

Figure 5-9: First Switch component - S1 


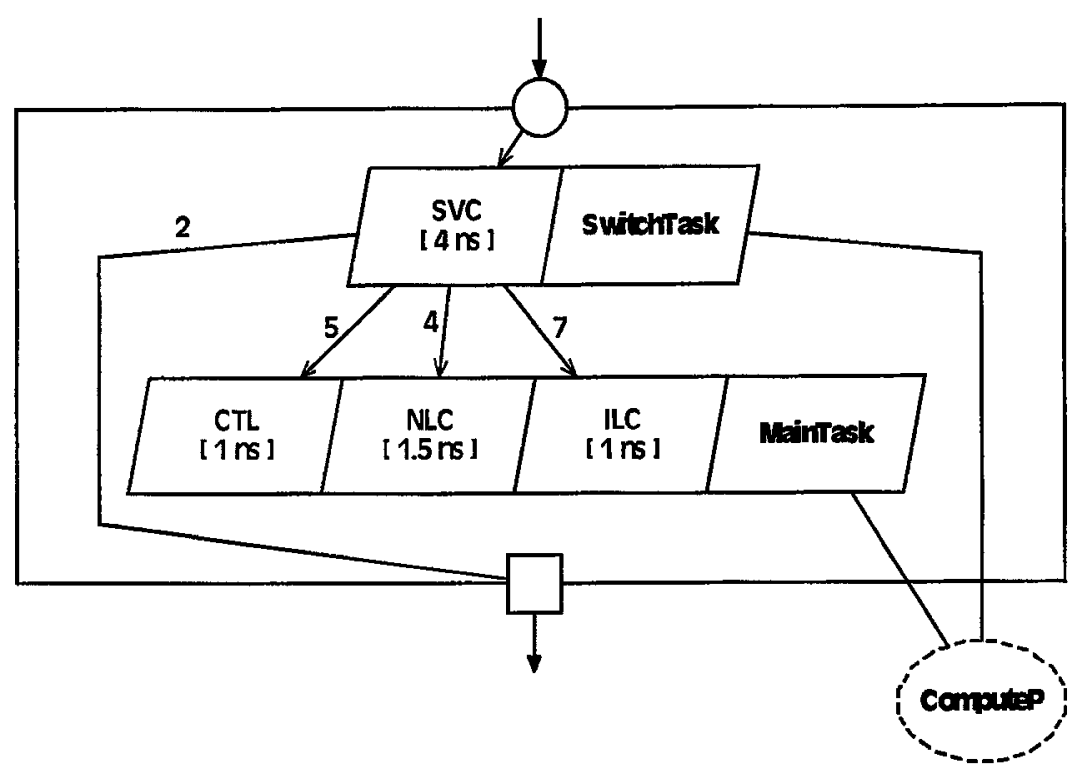

Figure 5-10: Second Switch component - S2

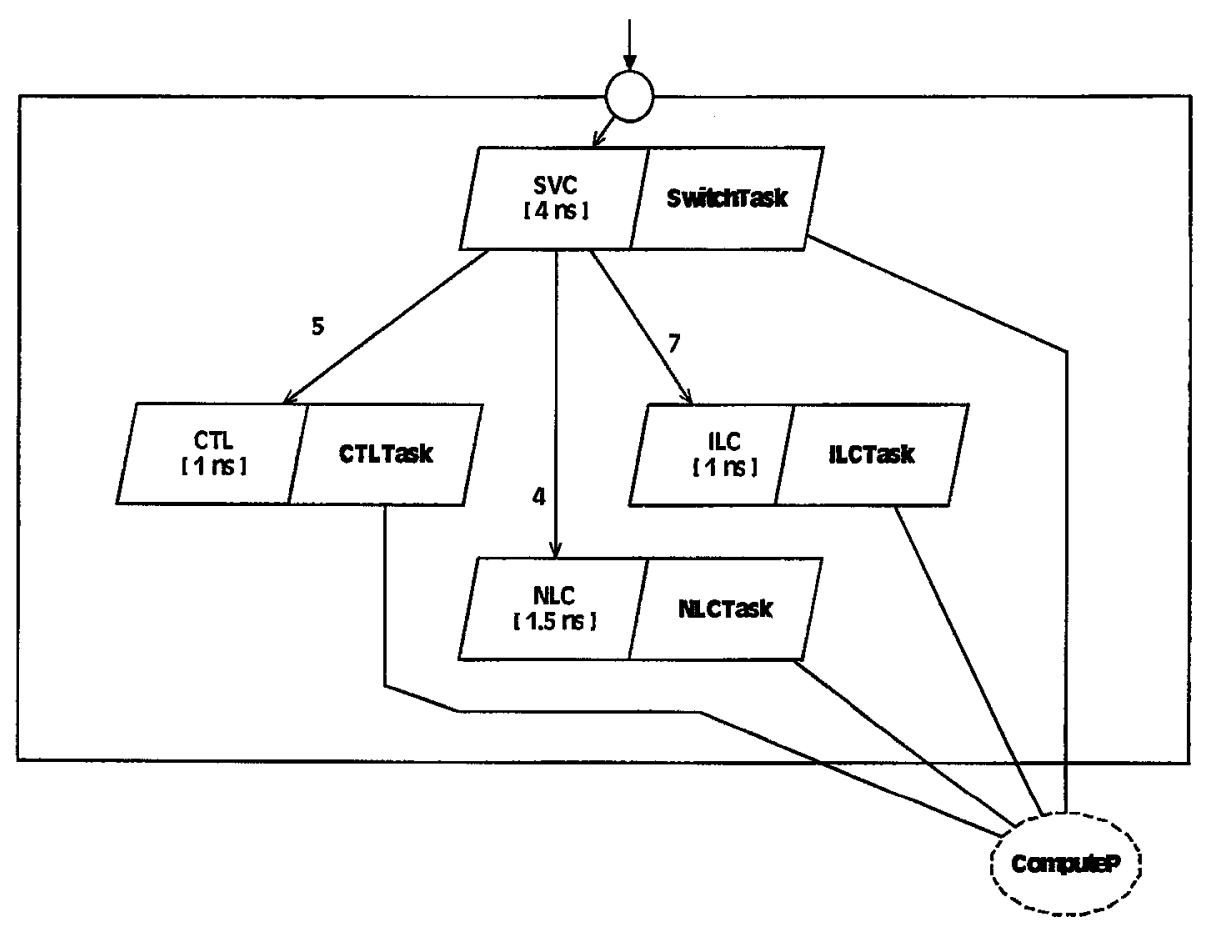

Figure 5-11: Third Switch component - S3 


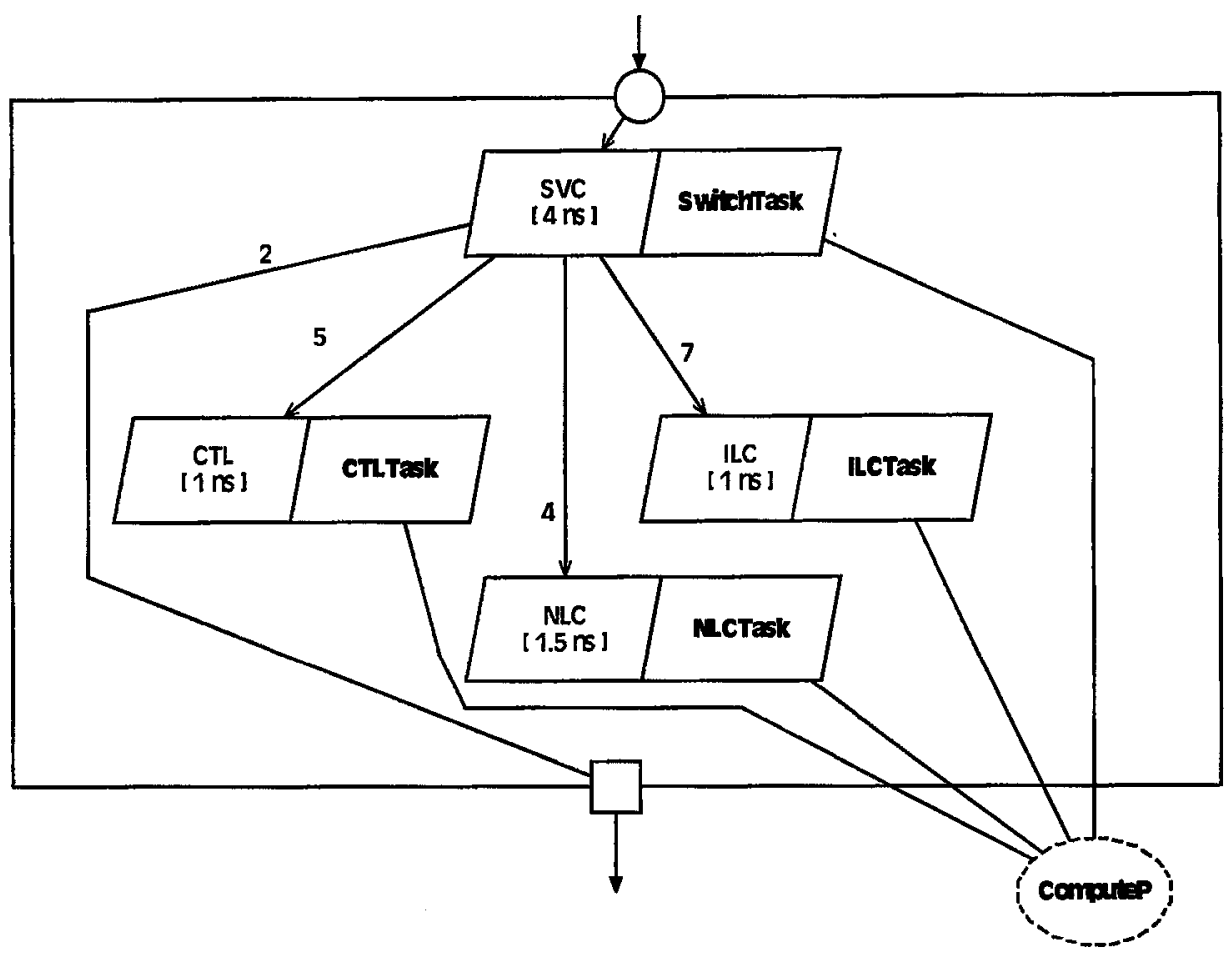

Figure 5-12: Fourth Switch component - S4

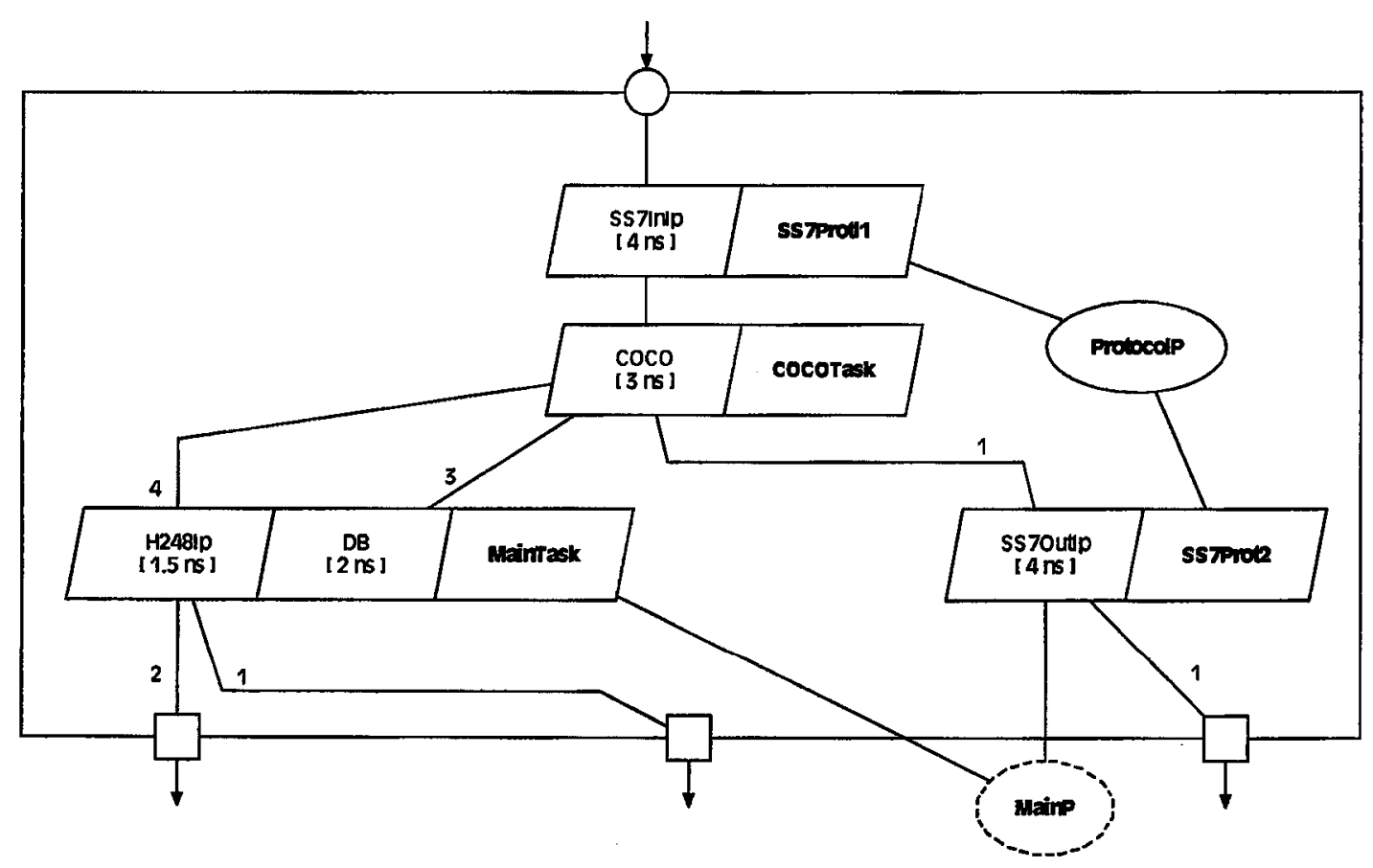

Figure 5-13: First Call Connection Agent component - C1 


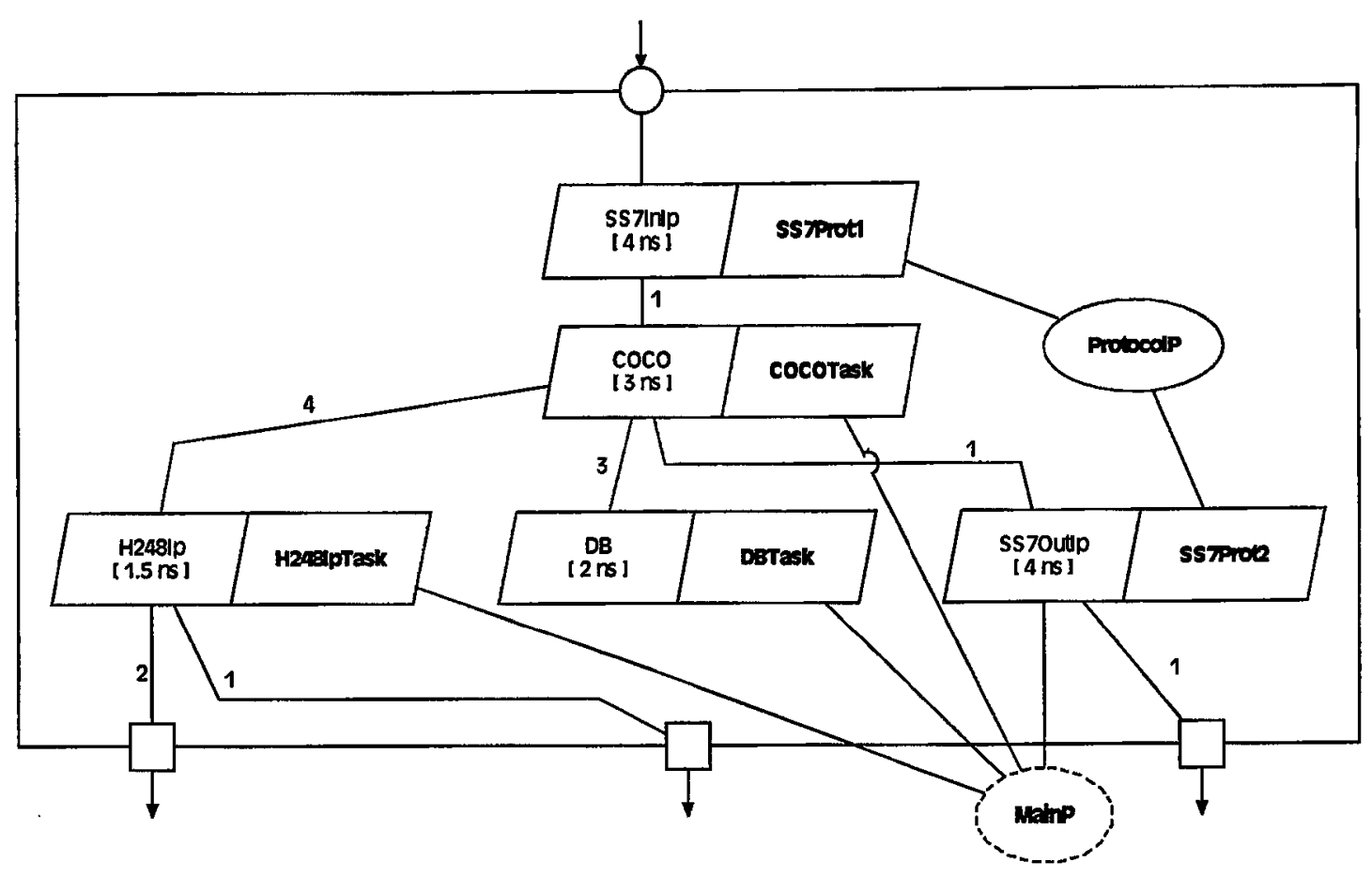

Figure 5-14: Second Call Connection Agent component - C2

Similar to gateway components, there are four different kinds of switch components. They are shown in Figure 5-9, Figure 5-10, Figure 5-11 and Figure 5-12. Of these, two of them are input only switches and the other two are input/output switches. The difference between them is that the operations CTL, NLC and ILC are bundled together in for the first and the third in Figure 5-9 and 5-10, and they are separated in Figure 5-11 and Figure 5-12.

Finally, Figure 5-13 and Figure 5-14 show the components proposed for the Call connection agent. The agents use the SS7 Protocol which is embedded in the SS7InIp and SS7OutIp tasks. The COCO agent makes a call to the Database, the H248Ip protocol and finally to the output task of protocol to be connected to class 5 switch. 
Bundling all the entries together reduces the demand on CPU but it has the disadvantage that since there is only one task to do all the three operations, it has the potential to slow down the system. On the other hand separating all the three operations into three different tasks increases the CPU demand but offers the advantage of concurrency.

\subsection{Building and Evaluating Model}

The components were added to the library and the system was put on auto-build to generate all the 64 possible alternatives. These alternatives are listed in the Table 5-5. The ones marked with " $\mathrm{X}$ " are the ones accepted by all hard constraints.

List of configurations

$\begin{array}{ll}\text { 1. } \mathrm{CC}=\mathrm{C} 1: \mathrm{TG}=\mathrm{G} 2: \mathrm{OG}=\mathrm{G} 1: \mathrm{TS}=\mathrm{S} 1: \mathrm{OS}=\mathrm{S} 2: \mathrm{CS}=\mathrm{S} 1 & \mathrm{X} \\ \text { 2. } \mathrm{CC}=\mathrm{C} 1: \mathrm{TG}=\mathrm{G} 2: \mathrm{OG}=\mathrm{G} 1: \mathrm{TS}=\mathrm{S} 1: \mathrm{OS}=\mathrm{S} 2: \mathrm{CS}=\mathrm{S} 3 & \mathrm{X} \\ \text { 3. } \mathrm{CC}=\mathrm{C} 1: \mathrm{TG}=\mathrm{G} 2: \mathrm{OG}=\mathrm{G} 1: \mathrm{TS}=\mathrm{S} 1: \mathrm{OS}=\mathrm{S} 4: \mathrm{CS}=\mathrm{S} 1 & \mathrm{X} \\ \text { 4. } \mathrm{CC}=\mathrm{C} 1: \mathrm{TG}=\mathrm{G} 2: \mathrm{OG}=\mathrm{G} 1: \mathrm{TS}=\mathrm{S} 1: \mathrm{OS}=\mathrm{S} 4: \mathrm{CS}=\mathrm{S} 3 & \\ \text { 5. } \mathrm{CC}=\mathrm{C} 1: \mathrm{TG}=\mathrm{G} 2: \mathrm{OG}=\mathrm{G} 1: \mathrm{TS}=\mathrm{S} 3: \mathrm{OS}=\mathrm{S} 2: \mathrm{CS}=\mathrm{S} 1 & \mathrm{X} \\ \text { 6. } \mathrm{CC}=\mathrm{C} 1: \mathrm{TG}=\mathrm{G} 2: \mathrm{OG}=\mathrm{G} 1: \mathrm{TS}=\mathrm{S} 3: \mathrm{OS}=\mathrm{S} 2: \mathrm{CS}=\mathrm{S} 3 & \mathrm{X} \\ \text { 7. } \mathrm{CC}=\mathrm{C} 1: \mathrm{TG}=\mathrm{G} 2: \mathrm{OG}=\mathrm{G} 1: \mathrm{TS}=\mathrm{S} 3: \mathrm{OS}=\mathrm{S} 4: \mathrm{CS}=\mathrm{S} 1 & \mathrm{X} \\ \text { 8. } \mathrm{CC}=\mathrm{C} 1: \mathrm{TG}=\mathrm{G} 2: \mathrm{OG}=\mathrm{G} 1: \mathrm{TS}=\mathrm{S} 3: \mathrm{OS}=\mathrm{S} 4: \mathrm{CS}=\mathrm{S} 3 & \\ \text { 9. } \mathrm{CC}=\mathrm{C} 1: \mathrm{TG}=\mathrm{G} 2: \mathrm{OG}=\mathrm{G} 3: \mathrm{TS}=\mathrm{S} 1: \mathrm{OS}=\mathrm{S} 2: \mathrm{CS}=\mathrm{S} 1 & \mathrm{X} \\ \text { 10. } \mathrm{CC}=\mathrm{C} 1: \mathrm{TG}=\mathrm{G} 2: \mathrm{OG}=\mathrm{G} 3: \mathrm{TS}=\mathrm{S} 1: \mathrm{OS}=\mathrm{S} 2: \mathrm{CS}=\mathrm{S} 3 & \mathrm{X} \\ \text { 11. } \mathrm{CC}=\mathrm{C} 1: \mathrm{TG}=\mathrm{G} 2: \mathrm{OG}=\mathrm{G} 3: \mathrm{TS}=\mathrm{S} 1: \mathrm{OS}=\mathrm{S} 4: \mathrm{CS}=\mathrm{S} 1 & \mathrm{X}\end{array}$




\begin{tabular}{|c|c|}
\hline \multicolumn{2}{|l|}{ 12. $\mathrm{CC}=\mathrm{C} 1: \mathrm{TG}=\mathrm{G} 2: \mathrm{OG}=\mathrm{G} 3: \mathrm{TS}=\mathrm{S} 1: \mathrm{OS}=\mathrm{S} 4: \mathrm{CS}=\mathrm{S} 3$} \\
\hline 13. $\mathrm{CC}=\mathrm{C} 1: \mathrm{TG}=\mathrm{G} 2: \mathrm{OG}=\mathrm{G} 3: \mathrm{TS}=\mathrm{S} 3: \mathrm{OS}=\mathrm{S} 2: \mathrm{CS}=\mathrm{S} 1$ & $\mathrm{X}$ \\
\hline 14. $\mathrm{CC}=\mathrm{C} 1: \mathrm{TG}=\mathrm{G} 2: \mathrm{OG}=\mathrm{G} 3: \mathrm{TS}=\mathrm{S} 3: \mathrm{OS}=\mathrm{S} 2: \mathrm{CS}=\mathrm{S} 3$ & $\mathrm{X}$ \\
\hline 15. $\mathrm{CC}=\mathrm{C} 1: \mathrm{TG}=\mathrm{G} 2: \mathrm{OG}=\mathrm{G} 3: \mathrm{TS}=\mathrm{S} 3: \mathrm{OS}=\mathrm{S} 4: \mathrm{CS}=\mathrm{S} 1$ & $\mathrm{X}$ \\
\hline \multicolumn{2}{|l|}{ 16. $\mathrm{CC}=\mathrm{C} 1: \mathrm{TG}=\mathrm{G} 2: \mathrm{OG}=\mathrm{G} 3: \mathrm{TS}=\mathrm{S} 3: \mathrm{OS}=\mathrm{S} 4: \mathrm{CS}=\mathrm{S} 3$} \\
\hline 17. $\mathrm{CC}=\mathrm{C} 1: \mathrm{TG}=\mathrm{G} 4: \mathrm{OG}=\mathrm{G} 1: \mathrm{TS}=\mathrm{S} 1: \mathrm{OS}=\mathrm{S} 2: \mathrm{CS}=\mathrm{S} 1$ & $\mathrm{X}$ \\
\hline \multicolumn{2}{|l|}{ 18. $\mathrm{CC}=\mathrm{C} 1: \mathrm{TG}=\mathrm{G} 4: \mathrm{OG}=\mathrm{G} 1: \mathrm{TS}=\mathrm{S} 1: \mathrm{OS}=\mathrm{S} 2: \mathrm{CS}=\mathrm{S} 3$} \\
\hline \multicolumn{2}{|l|}{ 19. $\mathrm{CC}=\mathrm{C} 1: \mathrm{TG}=\mathrm{G} 4: \mathrm{OG}=\mathrm{G} 1: \mathrm{TS}=\mathrm{S} 1: \mathrm{OS}=\mathrm{S} 4: \mathrm{CS}=\mathrm{S} 1$} \\
\hline \multicolumn{2}{|l|}{ 20. $\mathrm{CC}=\mathrm{C} 1: \mathrm{TG}=\mathrm{G} 4: \mathrm{OG}=\mathrm{G} 1: \mathrm{TS}=\mathrm{S} 1: \mathrm{OS}=\mathrm{S} 4: \mathrm{CS}=\mathrm{S} 3$} \\
\hline 21. $\mathrm{CC}=\mathrm{C} 1: \mathrm{TG}=\mathrm{G} 4: \mathrm{OG}=\mathrm{G} 1: \mathrm{TS}=\mathrm{S} 3: \mathrm{OS}=\mathrm{S} 2: \mathrm{CS}=\mathrm{S} 1$ & $\mathrm{X}$ \\
\hline \multicolumn{2}{|l|}{ 22. $\mathrm{CC}=\mathrm{C} 1: \mathrm{TG}=\mathrm{G} 4: \mathrm{OG}=\mathrm{G} 1: \mathrm{TS}=\mathrm{S} 3: \mathrm{OS}=\mathrm{S} 2: \mathrm{CS}=\mathrm{S} 3$} \\
\hline \multicolumn{2}{|l|}{ 23. $\mathrm{CC}=\mathrm{C} 1: \mathrm{TG}=\mathrm{G} 4: \mathrm{OG}=\mathrm{G} 1: \mathrm{TS}=\mathrm{S} 3: \mathrm{OS}=\mathrm{S} 4: \mathrm{CS}=\mathrm{S} 1$} \\
\hline \multicolumn{2}{|l|}{ 24. $\mathrm{CC}=\mathrm{C} 1: \mathrm{TG}=\mathrm{G} 4: \mathrm{OG}=\mathrm{G} 1: \mathrm{TS}=\mathrm{S} 3: \mathrm{OS}=\mathrm{S} 4: \mathrm{CS}=\mathrm{S} 3$} \\
\hline 25. $\mathrm{CC}=\mathrm{C} 1: \mathrm{TG}=\mathrm{G} 4: \mathrm{OG}=\mathrm{G} 3: \mathrm{TS}=\mathrm{S} 1: \mathrm{OS}=\mathrm{S} 2: \mathrm{CS}=\mathrm{S} 1$ & $\mathrm{x}$ \\
\hline \multicolumn{2}{|l|}{ 26. $\mathrm{CC}=\mathrm{C} 1: \mathrm{TG}=\mathrm{G} 4: \mathrm{OG}=\mathrm{G} 3: \mathrm{TS}=\mathrm{S} 1: \mathrm{OS}=\mathrm{S} 2: \mathrm{CS}=\mathrm{S} 3$} \\
\hline \multicolumn{2}{|l|}{ 27. $\mathrm{CC}=\mathrm{C} 1: \mathrm{TG}=\mathrm{G} 4: \mathrm{OG}=\mathrm{G} 3: \mathrm{TS}=\mathrm{S} 1: \mathrm{OS}=\mathrm{S} 4: \mathrm{CS}=\mathrm{S} 1$} \\
\hline \multicolumn{2}{|l|}{ 28. $\mathrm{CC}=\mathrm{C} 1: \mathrm{TG}=\mathrm{G} 4: \mathrm{OG}=\mathrm{G} 3: \mathrm{TS}=\mathrm{S} 1: \mathrm{OS}=\mathrm{S} 4: \mathrm{CS}=\mathrm{S} 3$} \\
\hline 29. $\mathrm{CC}=\mathrm{C} 1: \mathrm{TG}=\mathrm{G} 4: \mathrm{OG}=\mathrm{G} 3: \mathrm{TS}=\mathrm{S} 3: \mathrm{OS}=\mathrm{S} 2: \mathrm{CS}=\mathrm{S} 1$ & $\mathrm{X}$ \\
\hline \multicolumn{2}{|l|}{ 30. $\mathrm{CC}=\mathrm{C} 1: \mathrm{TG}=\mathrm{G} 4: \mathrm{OG}=\mathrm{G} 3: \mathrm{TS}=\mathrm{S} 3: \mathrm{OS}=\mathrm{S} 2: \mathrm{CS}=\mathrm{S} 3$} \\
\hline \multicolumn{2}{|l|}{ 31. $\mathrm{CC}=\mathrm{C} 1: \mathrm{TG}=\mathrm{G} 4: \mathrm{OG}=\mathrm{G} 3: \mathrm{TS}=\mathrm{S} 3: \mathrm{OS}=\mathrm{S} 4: \mathrm{CS}=\mathrm{S} 1$} \\
\hline \multicolumn{2}{|l|}{ 32. $\mathrm{CC}=\mathrm{C} 1: \mathrm{TG}=\mathrm{G} 4: \mathrm{OG}=\mathrm{G} 3: \mathrm{TS}=\mathrm{S} 3: \mathrm{OS}=\mathrm{S} 4: \mathrm{CS}=\mathrm{S} 3$} \\
\hline 33. $\mathrm{CC}=\mathrm{C} 2: \mathrm{TG}=\mathrm{G} 2: \mathrm{OG}=\mathrm{G} 1: \mathrm{TS}=\mathrm{S} 1: \mathrm{OS}=\mathrm{S} 2: \mathrm{CS}=\mathrm{S} 1$ & $\mathrm{X}$ \\
\hline 34. $\mathrm{CC}=\mathrm{C} 2: \mathrm{TG}=\mathrm{G} 2: \mathrm{OG}=\mathrm{G} 1: \mathrm{TS}=\mathrm{S} 1: \mathrm{OS}=\mathrm{S} 2: \mathrm{CS}=\mathrm{S} 3$ & $\mathrm{x}$ \\
\hline 35. $\mathrm{CC}=\mathrm{C} 2: \mathrm{TG}=\mathrm{G} 2: \mathrm{OG}=\mathrm{G} 1: \mathrm{TS}=\mathrm{S} 1: \mathrm{OS}=\mathrm{S} 4: \mathrm{CS}=\mathrm{S} 1$ & $X$ \\
\hline \multicolumn{2}{|l|}{ 36. $\mathrm{CC}=\mathrm{C} 2: \mathrm{TG}=\mathrm{G} 2: \mathrm{OG}=\mathrm{G} 1: \mathrm{TS}=\mathrm{S} 1: \mathrm{OS}=\mathrm{S} 4: \mathrm{CS}=\mathrm{S} 3$} \\
\hline 37. $\mathrm{CC}=\mathrm{C} 2: \mathrm{TG}=\mathrm{G} 2: \mathrm{OG}=\mathrm{G} 1: \mathrm{TS}=\mathrm{S} 3: \mathrm{OS}=\mathrm{S} 2: \mathrm{CS}=\mathrm{S} 1$ & $\mathrm{x}$ \\
\hline 38. $\mathrm{CC}=\mathrm{C} 2: \mathrm{TG}=\mathrm{G} 2: \mathrm{OG}=\mathrm{G} 1: \mathrm{TS}=\mathrm{S} 3: \mathrm{OS}=\mathrm{S} 2: \mathrm{CS}=\mathrm{S} 3$ & $\mathrm{x}$ \\
\hline
\end{tabular}




\begin{tabular}{|c|c|}
\hline 39. $\mathrm{CC}=\mathrm{C} 2: \mathrm{TG}=\mathrm{G} 2: \mathrm{OG}=\mathrm{G} 1: \mathrm{TS}=\mathrm{S} 3: \mathrm{OS}=\mathrm{S} 4: \mathrm{CS}=\mathrm{S} 1$ & $\mathrm{X}$ \\
\hline \multicolumn{2}{|l|}{ 40. $\mathrm{CC}=\mathrm{C} 2: \mathrm{TG}=\mathrm{G} 2: \mathrm{OG}=\mathrm{G} 1: \mathrm{TS}=\mathrm{S} 3: \mathrm{OS}=\mathrm{S} 4: \mathrm{CS}=\mathrm{S} 3$} \\
\hline 41. $\mathrm{CC}=\mathrm{C} 2: \mathrm{TG}=\mathrm{G} 2: \mathrm{OG}=\mathrm{G} 3: \mathrm{TS}=\mathrm{S} 1: \mathrm{OS}=\mathrm{S} 2: \mathrm{CS}=\mathrm{S} 1$ & $\mathrm{X}$ \\
\hline 42. $\mathrm{CC}=\mathrm{C} 2: \mathrm{TG}=\mathrm{G} 2: \mathrm{OG}=\mathrm{G} 3: \mathrm{TS}=\mathrm{S} 1: \mathrm{OS}=\mathrm{S} 2: \mathrm{CS}=\mathrm{S} 3$ & $\mathrm{X}$ \\
\hline 43. $\mathrm{CC}=\mathrm{C} 2: \mathrm{TG}=\mathrm{G} 2: \mathrm{OG}=\mathrm{G} 3: \mathrm{TS}=\mathrm{S} 1: \mathrm{OS}=\mathrm{S} 4: \mathrm{CS}=\mathrm{S} 1$ & $\mathrm{X}$ \\
\hline \multicolumn{2}{|l|}{ 44. $\mathrm{CC}=\mathrm{C} 2: \mathrm{TG}=\mathrm{G} 2: \mathrm{OG}=\mathrm{G} 3: \mathrm{TS}=\mathrm{S} 1: \mathrm{OS}=\mathrm{S} 4: \mathrm{CS}=\mathrm{S} 3$} \\
\hline 45. $\mathrm{CC}=\mathrm{C} 2: \mathrm{TG}=\mathrm{G} 2: \mathrm{OG}=\mathrm{G} 3: \mathrm{TS}=\mathrm{S} 3: \mathrm{OS}=\mathrm{S} 2: \mathrm{CS}=\mathrm{S} 1$ & $\mathbf{X}$ \\
\hline 46. $\mathrm{CC}=\mathrm{C} 2: \mathrm{TG}=\mathrm{G} 2: \mathrm{OG}=\mathrm{G} 3: \mathrm{TS}=\mathrm{S} 3: \mathrm{OS}=\mathrm{S} 2: \mathrm{CS}=\mathrm{S} 3$ & $\mathrm{x}$ \\
\hline 47. $\mathrm{CC}=\mathrm{C} 2: \mathrm{TG}=\mathrm{G} 2: \mathrm{OG}=\mathrm{G} 3: \mathrm{TS}=\mathrm{S} 3: \mathrm{OS}=\mathrm{S} 4: \mathrm{CS}=\mathrm{S} 1$ & $\mathrm{X}$ \\
\hline \multicolumn{2}{|l|}{ 48. $\mathrm{CC}=\mathrm{C} 2: \mathrm{TG}=\mathrm{G} 2: \mathrm{OG}=\mathrm{G} 3: \mathrm{TS}=\mathrm{S} 3: \mathrm{OS}=\mathrm{S} 4: \mathrm{CS}=\mathrm{S} 3$} \\
\hline 49. $\mathrm{CC}=\mathrm{C} 2: \mathrm{TG}=\mathrm{G} 4: \mathrm{OG}=\mathrm{G} 1: \mathrm{TS}=\mathrm{S} 1: \mathrm{OS}=\mathrm{S} 2: \mathrm{CS}=\mathrm{S} 1$ & $\mathrm{X}$ \\
\hline \multicolumn{2}{|l|}{ 50. $\mathrm{CC}=\mathrm{C} 2: \mathrm{TG}=\mathrm{G} 4: \mathrm{OG}=\mathrm{G} 1: \mathrm{TS}=\mathrm{S} 1: \mathrm{OS}=\mathrm{S} 2: \mathrm{CS}=\mathrm{S} 3$} \\
\hline \multicolumn{2}{|l|}{ 51. $\mathrm{CC}=\mathrm{C} 2: \mathrm{TG}=\mathrm{G} 4: \mathrm{OG}=\mathrm{G} 1: \mathrm{TS}=\mathrm{S} 1: \mathrm{OS}=\mathrm{S} 4: \mathrm{CS}=\mathrm{S} 1$} \\
\hline \multicolumn{2}{|l|}{ 52. $\mathrm{CC}=\mathrm{C} 2: \mathrm{TG}=\mathrm{G} 4: \mathrm{OG}=\mathrm{G} 1: \mathrm{TS}=\mathrm{S} 1: \mathrm{OS}=\mathrm{S} 4: \mathrm{CS}=\mathrm{S} 3$} \\
\hline 53. $\mathrm{CC}=\mathrm{C} 2: \mathrm{TG}=\mathrm{G} 4: \mathrm{OG}=\mathrm{G} 1: \mathrm{TS}=\mathrm{S} 3: \mathrm{OS}=\mathrm{S} 2: \mathrm{CS}=\mathrm{S} 1$ & $\mathrm{X}$ \\
\hline \multicolumn{2}{|l|}{ 54. $\mathrm{CC}=\mathrm{C} 2: \mathrm{TG}=\mathrm{G} 4: \mathrm{OG}=\mathrm{G} 1: \mathrm{TS}=\mathrm{S} 3: \mathrm{OS}=\mathrm{S} 2: \mathrm{CS}=\mathrm{S} 3$} \\
\hline \multicolumn{2}{|l|}{ 55. $\mathrm{CC}=\mathrm{C} 2: \mathrm{TG}=\mathrm{G} 4: \mathrm{OG}=\mathrm{G} 1: \mathrm{TS}=\mathrm{S} 3: \mathrm{OS}=\mathrm{S} 4: \mathrm{CS}=\mathrm{S} 1$} \\
\hline \multicolumn{2}{|l|}{ 56. $\mathrm{CC}=\mathrm{C} 2: \mathrm{TG}=\mathrm{G} 4: \mathrm{OG}=\mathrm{G} 1: \mathrm{TS}=\mathrm{S} 3: \mathrm{OS}=\mathrm{S} 4: \mathrm{CS}=\mathrm{S} 3$} \\
\hline 57. $\mathrm{CC}=\mathrm{C} 2: \mathrm{TG}=\mathrm{G} 4: \mathrm{OG}=\mathrm{G} 3: \mathrm{TS}=\mathrm{S} 1: \mathrm{OS}=\mathrm{S} 2: \mathrm{CS}=\mathrm{S} 1$ & $\mathrm{X}$ \\
\hline \multicolumn{2}{|l|}{ 58. $\mathrm{CC}=\mathrm{C} 2: \mathrm{TG}=\mathrm{G} 4: \mathrm{OG}=\mathrm{G} 3: \mathrm{TS}=\mathrm{S} 1: \mathrm{OS}=\mathrm{S} 2: \mathrm{CS}=\mathrm{S} 3$} \\
\hline \multicolumn{2}{|l|}{ 59. $\mathrm{CC}=\mathrm{C} 2: \mathrm{TG}=\mathrm{G} 4: \mathrm{OG}=\mathrm{G} 3: \mathrm{TS}=\mathrm{S} 1: \mathrm{OS}=\mathrm{S} 4: \mathrm{CS}=\mathrm{S} 1$} \\
\hline \multicolumn{2}{|l|}{ 60. $\mathrm{CC}=\mathrm{C} 2: \mathrm{TG}=\mathrm{G} 4: \mathrm{OG}=\mathrm{G} 3: \mathrm{TS}=\mathrm{S} 1: \mathrm{OS}=\mathrm{S} 4: \mathrm{CS}=\mathrm{S} 3$} \\
\hline 61. $\mathrm{CC}=\mathrm{C} 2: \mathrm{TG}=\mathrm{G} 4: \mathrm{OG}=\mathrm{G} 3: \mathrm{TS}=\mathrm{S} 3: \mathrm{OS}=\mathrm{S} 2: \mathrm{CS}=\mathrm{S} 1$ & $\mathrm{X}$ \\
\hline \multicolumn{2}{|l|}{ 62. $\mathrm{CC}=\mathrm{C} 2: \mathrm{TG}=\mathrm{G} 4: \mathrm{OG}=\mathrm{G} 3: \mathrm{TS}=\mathrm{S} 3: \mathrm{OS}=\mathrm{S} 2: \mathrm{CS}=\mathrm{S} 3$} \\
\hline 63. $\mathrm{CC}=\mathrm{C} 2: \mathrm{TG}=\mathrm{G} 4: \mathrm{OG}=\mathrm{G} 3: \mathrm{TS}=\mathrm{S} 3: \mathrm{OS}=\mathrm{S} 4: \mathrm{CS}=\mathrm{S} 1$ & \\
\hline 64. $\mathrm{CC}=\mathrm{C} 2: \mathrm{TG}=\mathrm{G} 4: \mathrm{OG}=\mathrm{G} 3: \mathrm{TS}=\mathrm{S} 3: \mathrm{OS}=\mathrm{S} 4: \mathrm{CS}=\mathrm{S} 3$ & \\
\hline
\end{tabular}

Table 5-5: List of all configurations generated by the tool 
These alternatives were evaluated against the hard constraints first. The results are shown in the Figures 5-15, 5-16 and 5-17. Figure 5-15 shows all the alternatives evaluated against the first hard constraint on throughput. There is a bar at 0.5 which divides the chart into two areas - the acceptable area and the unacceptable area. The alternatives for which the throughput values which lie in the unacceptable region are rejected and those in the acceptable region are carried forward.

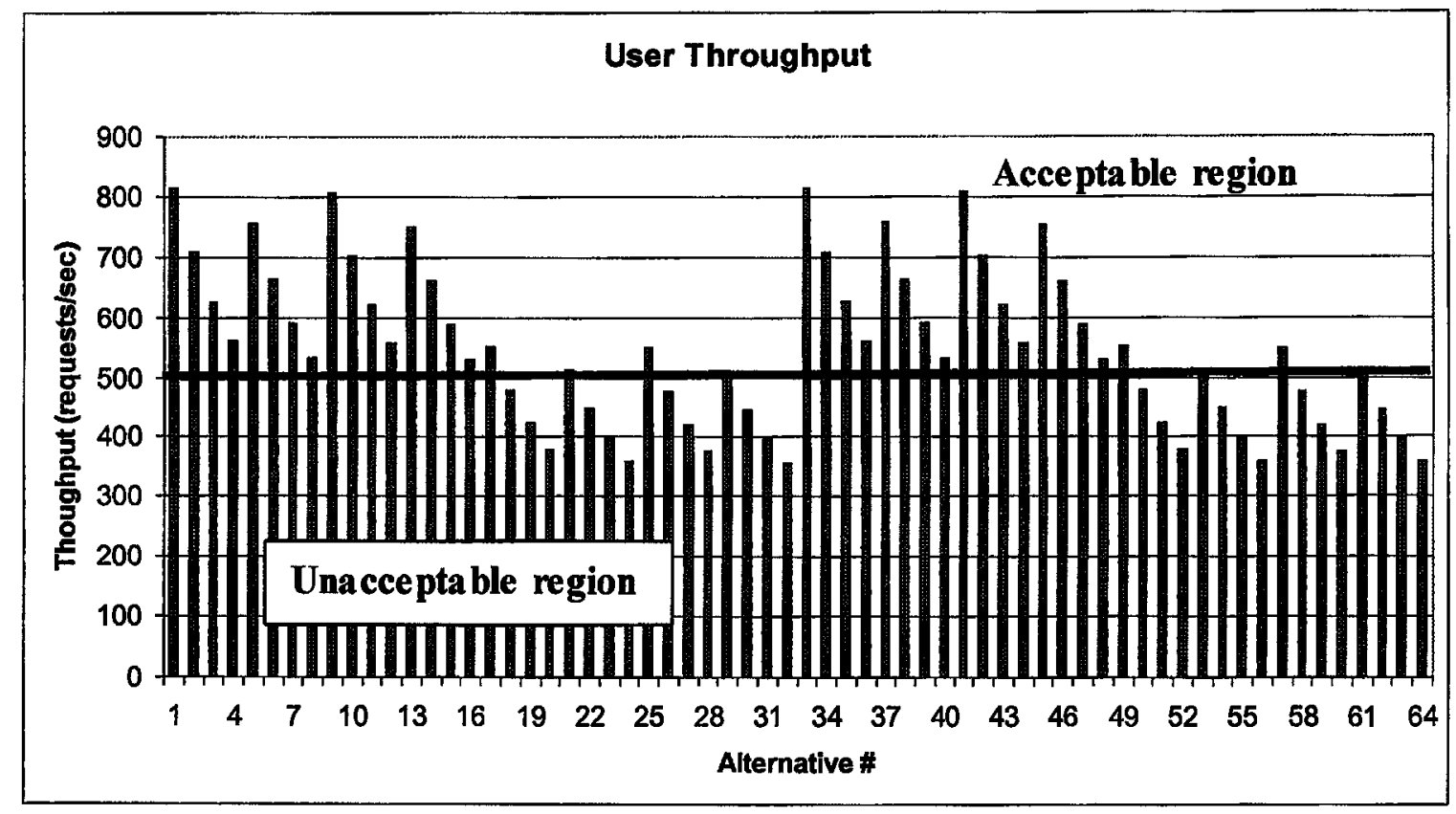

Figure 5-15: User throughput values for all the alternatives

Figure 5-16 shows all the alternatives evaluated against the second hard constraint on response time. The bar in this case is at 2.000 which divide the chart into the acceptable area and the unacceptable area. The alternatives for which response time lie above $2 \mathrm{sec}$. are in the unacceptable region and are rejected and those in the acceptable region are carried forward. 


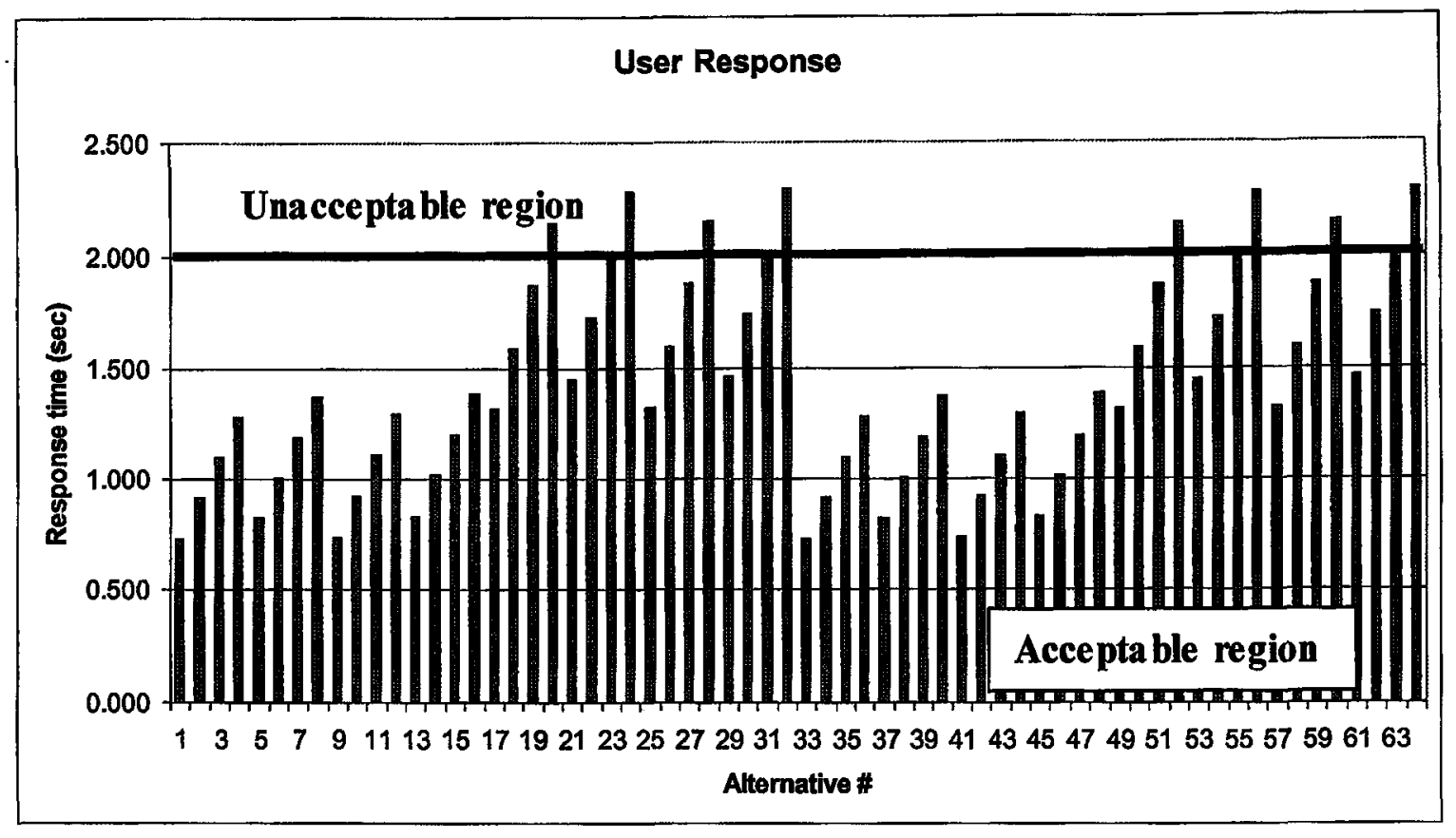

Figure 5-16: User response time for all the alternatives

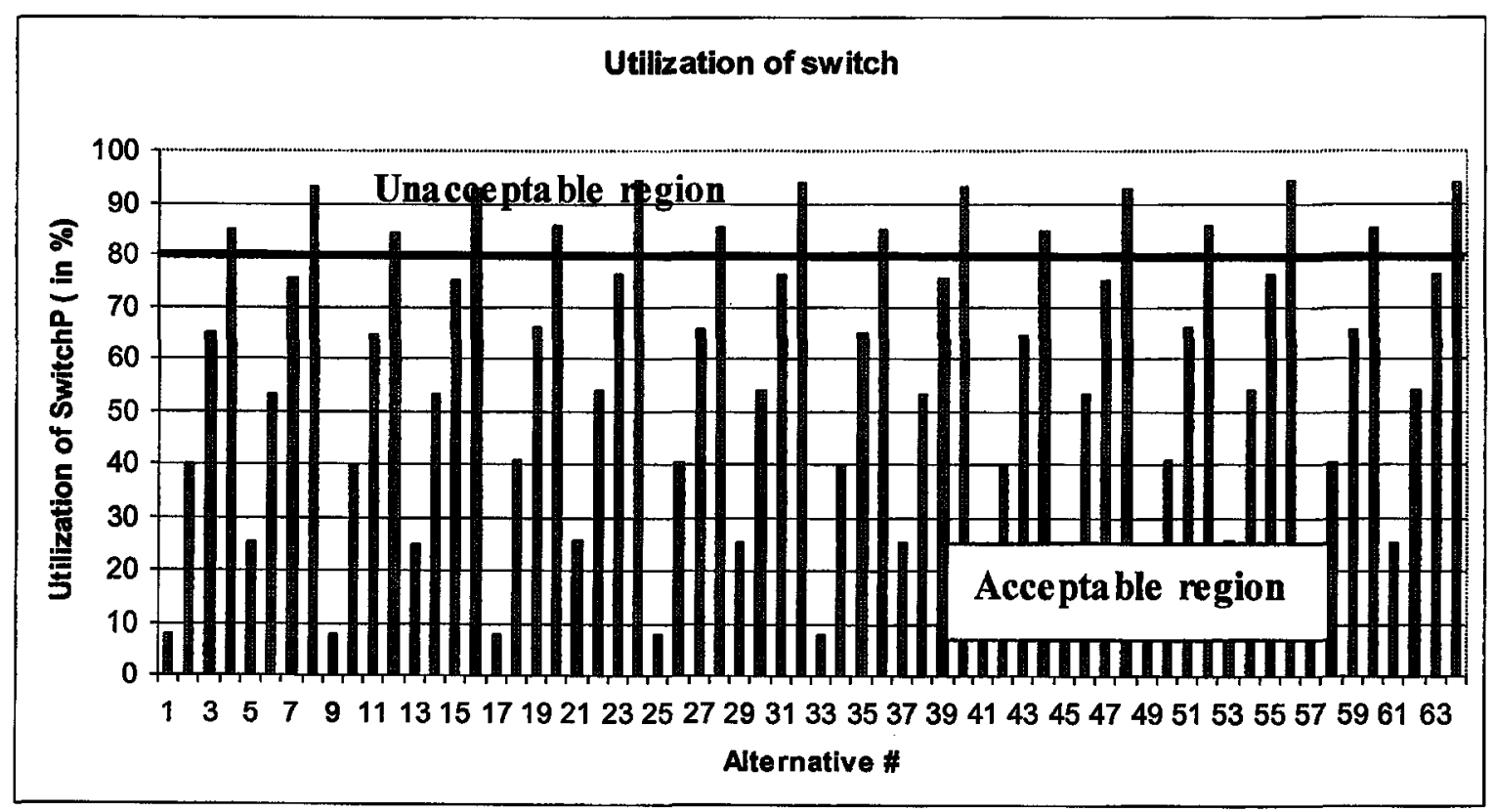

Figure 5-17: User throughput values for all the alternatives 
Figure 5-17 shows all the alternatives evaluated against the third hard constraint on utilization of SwitchP processor. The bar here is at $50 \%$ dividing the chart into the acceptable and unacceptable areas. The alternatives for which utilization of SwitchP are above $50 \%$ are rejected.

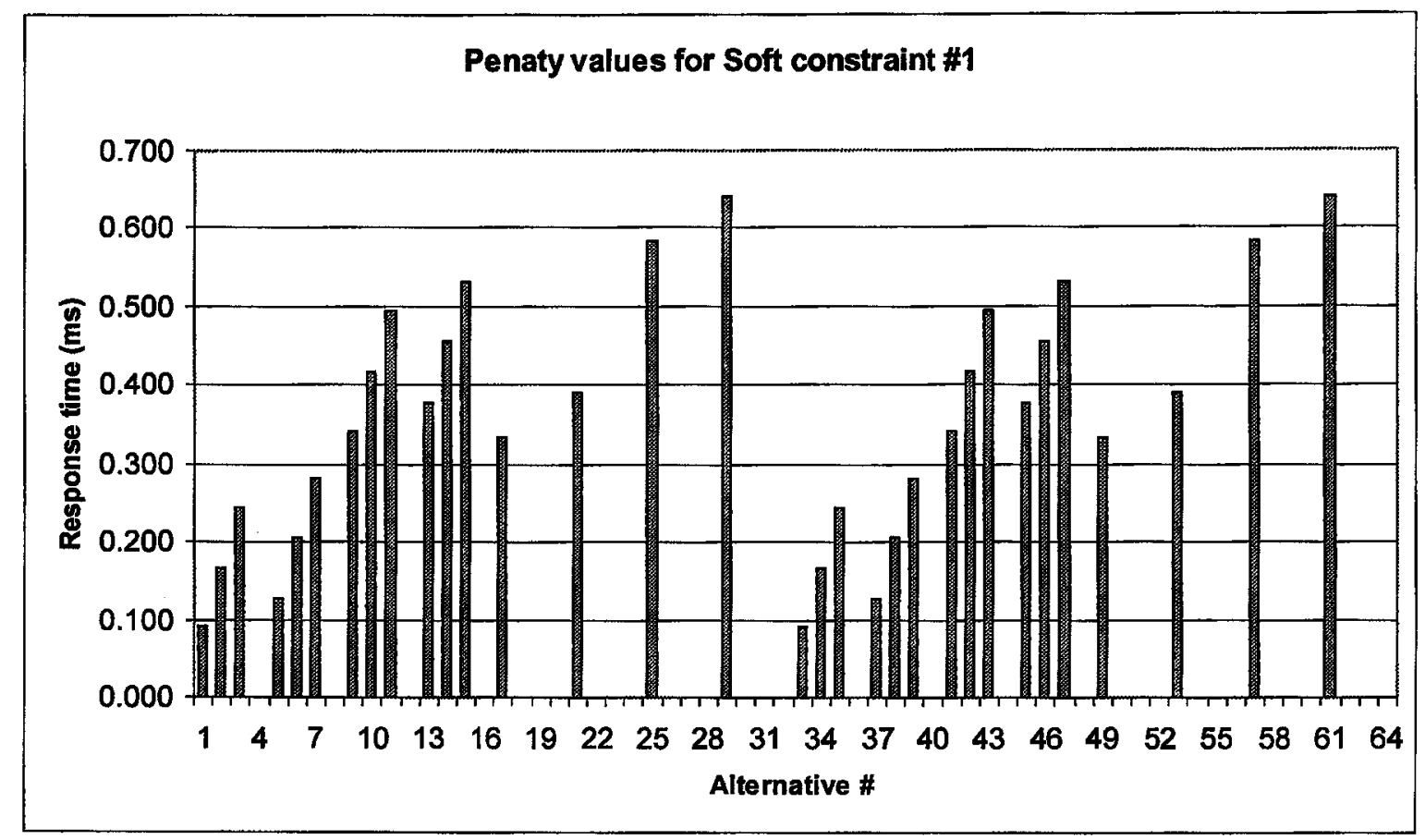

Figure 5-18: Penalty value ( $\left.p_{i}\right)$ of soft constraint $\# 1$ on alternatives

All of the alternatives which satisfy all three hard constraints are retained. There are 32 alternatives that are retained and all other are rejected as shown in Table 5-5. Of these 32 alternatives, soft constraints are applied and their results are shown in Figure 5-18, Figure 5-19 and Figure 5-20. Figure 5-18 shows application of first soft constraint (Table 5-2), which is a linear combination of response times of originating gateway and terminal 
gateway, on all selected alternatives. The penalty value for this constraint is calculated as follows:

$$
\begin{aligned}
& a_{i}=R_{\text {TermGateway }}+30 \times R_{\text {OrigGateway }} \\
& p_{i}=\left\{\begin{array}{c}
0 \quad \text { if } a_{i}<500 \\
a_{i} / 500^{-1} \text { if } a_{i} \geq 500
\end{array}\right.
\end{aligned}
$$

Figure 5-19 shows application of second soft constraint (Table 5-2), which is a linear combination of utilization of processors GatewayP and MainP, on all selected alternatives. The penalty value for this constraint is calculated as follows:

$$
\begin{aligned}
& b_{i}=U_{\text {Gateway } P}+8 \times U_{\text {MainP }} \\
& q_{i}=\left\{\begin{array}{c}
0 \quad \text { if } b_{i}<16 \\
b_{i} / 16-1 \text { if } b_{i} \geq 16
\end{array}\right.
\end{aligned}
$$

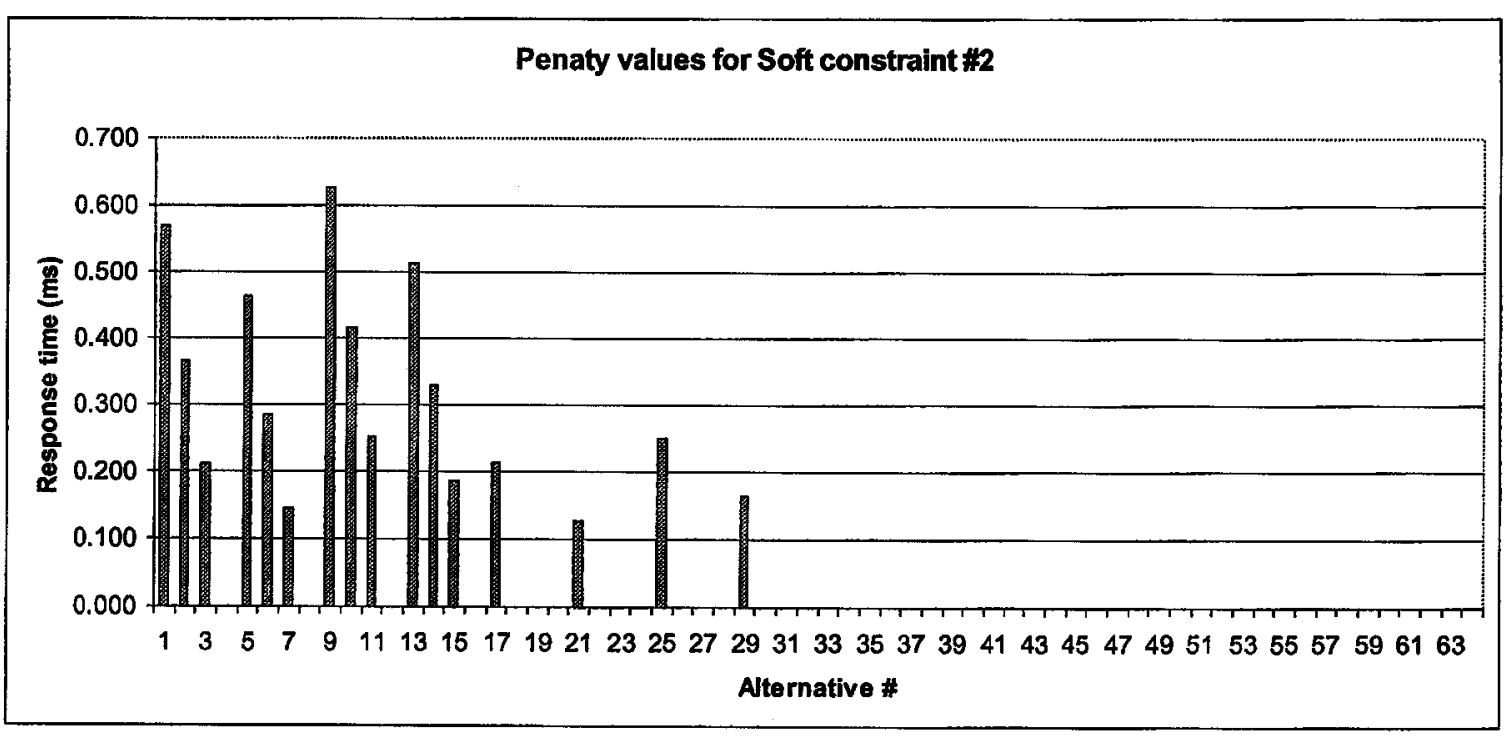

Figure 5-19: Penalty value ( $\left.q_{i}\right)$ of soft constraint $\# 2$ on alternatives

Figure 5-20 shows application of third soft constraint (Table 5-3), which a constraint on response time of core switch. The penalty value for this constraint is calculated as follows: 


$$
r_{i}=\left\{\begin{array}{c}
0 \\
R_{\text {CoreSW }} / 65-r_{\text {if }}-R_{\text {CoresW }}<65 \\
\text { if } R_{\text {CoreSW }} \geq 65
\end{array}\right.
$$

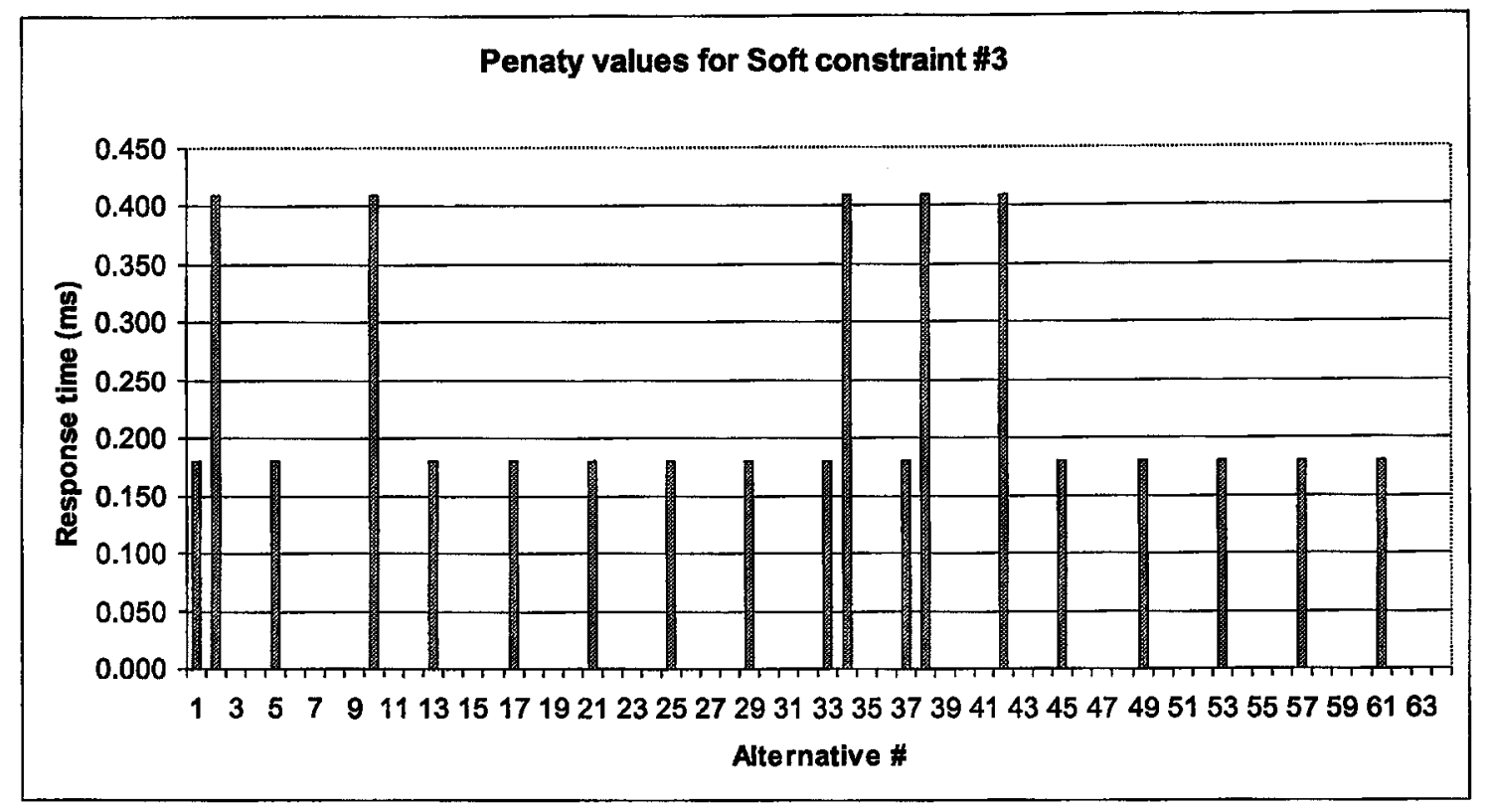

Figure 5-20: Penalty value $\left(r_{i}\right)$ of soft constraint \#3 on alternatives

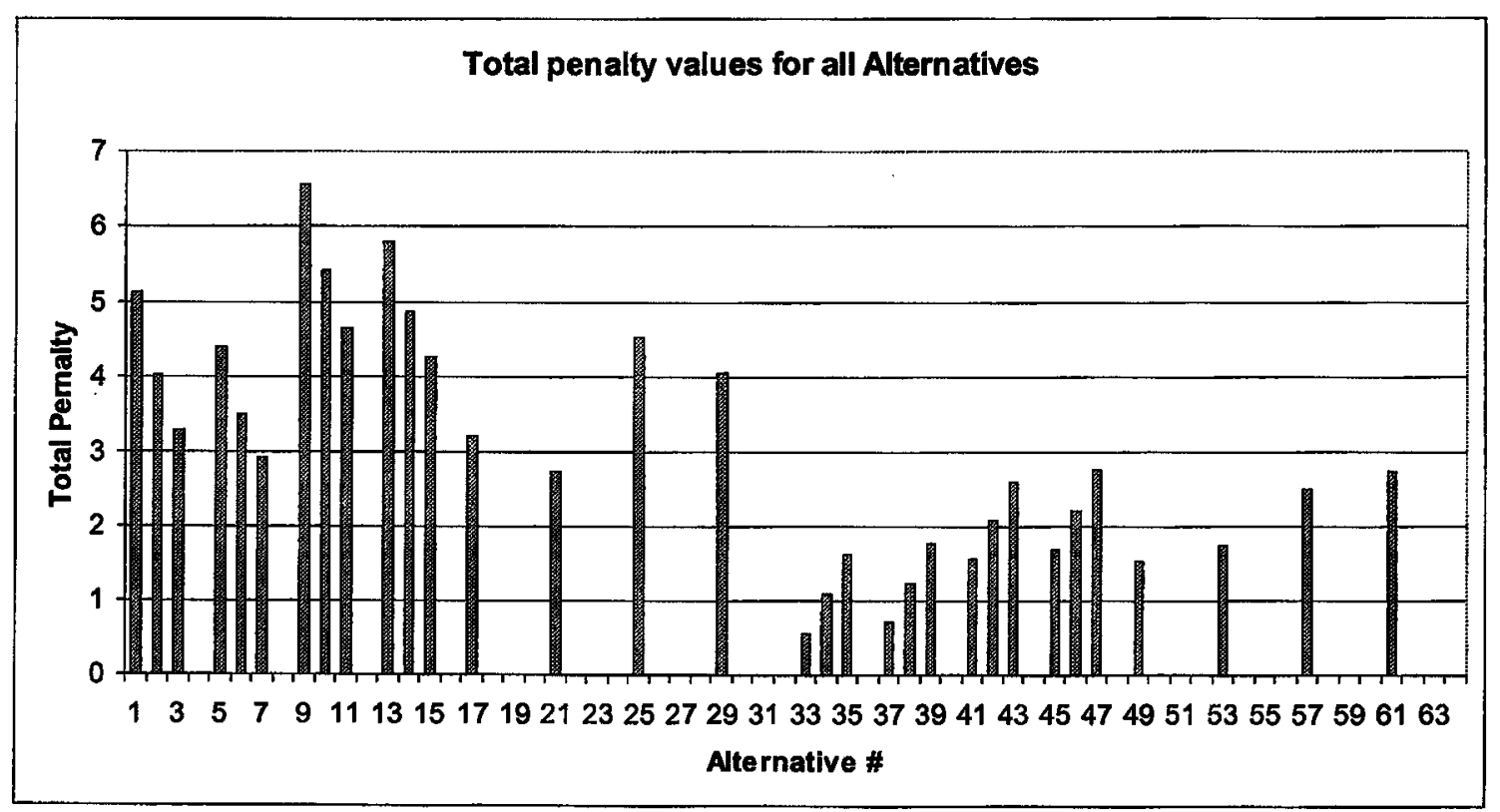

Figure 5-21: Penalty value $\left(f_{i}\right)$ of all constraints on alternatives 
Finally we combine all the penalty values in their respective weights to get the total penalty value for all the alternatives. This is shown in Figure 5-21 and is simply calculated by combining all the penalty values in their respective weights as follows:

$$
f_{i}=4 \times p_{i}+8 \times q_{i}+1 \times r_{i}
$$

\subsection{Interpreting the Results}

We obtain the total penalty value over all soft constraints for all alternatives. These are summarized in Table 5-6. This gives us the ranking of alternatives from a performance point of view, with the best alternative first.

\begin{tabular}{c|c|c|c}
\hline Rank & Alternative\# & \multicolumn{1}{|c|}{ Configuration } & $\begin{array}{c}\text { Total penalty } \\
\text { value }\end{array}$ \\
\hline 1 & 33 & $\mathrm{CC}=\mathrm{C} 2: \mathrm{TG}=\mathrm{G} 2: \mathrm{OG}=\mathrm{G} 1: \mathrm{TS}=\mathrm{S} 1: \mathrm{OS}=\mathrm{S} 2: \mathrm{CS}=\mathrm{S} 1$ & 0.54 \\
2 & 37 & $\mathrm{CC}=\mathrm{C} 2: \mathrm{TG}=\mathrm{G} 2: \mathrm{OG}=\mathrm{G} 1: \mathrm{TS}=\mathrm{S} 3: \mathrm{OS}=\mathrm{S} 2: \mathrm{CS}=\mathrm{S} 1$ & 0.693 \\
3 & 34 & $\mathrm{CC}=\mathrm{C} 2: \mathrm{TG}=\mathrm{G} 2: \mathrm{OG}=\mathrm{G} 1: \mathrm{TS}=\mathrm{S} 1: \mathrm{OS}=\mathrm{S} 2: \mathrm{CS}=\mathrm{S} 3$ & 1.077 \\
4 & 38 & $\mathrm{CC}=\mathrm{C} 2: \mathrm{TG}=\mathrm{G} 2: \mathrm{OG}=\mathrm{G} 1: \mathrm{TS}=\mathrm{S} 3: \mathrm{OS}=\mathrm{S} 2: \mathrm{CS}=\mathrm{S} 3$ & 1.23 \\
5 & 49 & $\mathrm{CC}=\mathrm{C} 2: \mathrm{TG}=\mathrm{G} 4: \mathrm{OG}=\mathrm{G} 1: \mathrm{TS}=\mathrm{S} 1: \mathrm{OS}=\mathrm{S} 2: \mathrm{CS}=\mathrm{S} 1$ & 1.513 \\
6 & 41 & $\mathrm{CC}=\mathrm{C} 2: \mathrm{TG}=\mathrm{G} 2: \mathrm{OG}=\mathrm{G} 3: \mathrm{TS}=\mathrm{S} 1: \mathrm{OS}=\mathrm{S} 2: \mathrm{CS}=\mathrm{S} 1$ & 1.54 \\
7 & 35 & $\mathrm{CC}=\mathrm{C} 2: \mathrm{TG}=\mathrm{G} 2: \mathrm{OG}=\mathrm{G} 1: \mathrm{TS}=\mathrm{S} 1: \mathrm{OS}=\mathrm{S} 4: \mathrm{CS}=\mathrm{S} 1$ & 1.613 \\
8 & 45 & $\mathrm{CC}=\mathrm{C} 2: \mathrm{TG}=\mathrm{G} 2: \mathrm{OG}=\mathrm{G} 3: \mathrm{TS}=\mathrm{S} 3: \mathrm{OS}=\mathrm{S} 2: \mathrm{CS}=\mathrm{S} 1$ & 1.693 \\
9 & 53 & $\mathrm{CC}=\mathrm{C} 2: \mathrm{TG}=\mathrm{G} 4: \mathrm{OG}=\mathrm{G} 1: \mathrm{TS}=\mathrm{S} 3: \mathrm{OS}=\mathrm{S} 2: \mathrm{CS}=\mathrm{S} 1$ & 1.743 \\
10 & 39 & $\mathrm{CC}=\mathrm{C} 2: \mathrm{TG}=\mathrm{G} 2: \mathrm{OG}=\mathrm{G} 1: \mathrm{TS}=\mathrm{S} 3: \mathrm{OS}=\mathrm{S} 4: \mathrm{CS}=\mathrm{S} 1$ & 1.767 \\
11 & 42 & $\mathrm{CC}=\mathrm{C} 2: \mathrm{TG}=\mathrm{G} 2: \mathrm{OG}=\mathrm{G} 3: \mathrm{TS}=\mathrm{S} 1: \mathrm{OS}=\mathrm{S} 2: \mathrm{CS}=\mathrm{S} 3$ & 2.077 \\
12 & 46 & $\mathrm{CC}=\mathrm{C} 2: \mathrm{TG}=\mathrm{G} 2: \mathrm{OG}=\mathrm{G} 3: \mathrm{TS}=\mathrm{S} 3: \mathrm{OS}=\mathrm{S} 2: \mathrm{CS}=\mathrm{S} 3$ & 2.23 \\
13 & 57 & $\mathrm{CC}=\mathrm{C} 2: \mathrm{TG}=\mathrm{G} 4: \mathrm{OG}=\mathrm{G} 3: \mathrm{TS}=\mathrm{S} 1: \mathrm{OS}=\mathrm{S} 2: \mathrm{CS}=\mathrm{S} 1$ & 2.513 \\
14 & 43 & $\mathrm{CC}=\mathrm{C} 2: \mathrm{TG}=\mathrm{G} 2: \mathrm{OG}=\mathrm{G} 3: \mathrm{TS}=\mathrm{S} 1: \mathrm{OS}=\mathrm{S} 4: \mathrm{CS}=\mathrm{S} 1$ & 2.613 \\
15 & 61 & $\mathrm{CC}=\mathrm{C} 2: \mathrm{TG}=\mathrm{G} 4: \mathrm{OG}=\mathrm{G} 3: \mathrm{TS}=\mathrm{S} 3: \mathrm{OS}=\mathrm{S} 2: \mathrm{CS}=\mathrm{S} 1$ & 2.743 \\
16 & 21 & $\mathrm{CC}=\mathrm{C} 1: \mathrm{TG}=\mathrm{G} 4: \mathrm{OG}=\mathrm{G} 1: \mathrm{TS}=\mathrm{S} 3: \mathrm{OS}=\mathrm{S} 2: \mathrm{CS}=\mathrm{S} 1$ & 2.755 \\
& & & \\
& 4 & &
\end{tabular}




\begin{tabular}{l|c|l|l}
17 & 47 & $\mathrm{CC}=\mathrm{C} 2: \mathrm{TG}=\mathrm{G} 2: \mathrm{OG}=\mathrm{G} 3: \mathrm{TS}=\mathrm{S} 3: \mathrm{OS}=\mathrm{S} 4: \mathrm{CS}=\mathrm{S} 1$ & 2.767 \\
18 & 7 & $\mathrm{CC}=\mathrm{C} 1: \mathrm{TG}=\mathrm{G} 2: \mathrm{OG}=\mathrm{G} 1: \mathrm{TS}=\mathrm{S} 3: \mathrm{OS}=\mathrm{S} 4: \mathrm{CS}=\mathrm{S} 1$ & 2.914 \\
19 & 17 & $\mathrm{CC}=\mathrm{C} 1: \mathrm{TG}=\mathrm{G} 4: \mathrm{OG}=\mathrm{G} 1: \mathrm{TS}=\mathrm{S} 1: \mathrm{OS}=\mathrm{S} 2: \mathrm{CS}=\mathrm{S} 1$ & 3.211 \\
20 & 3 & $\mathrm{CC}=\mathrm{C} 1: \mathrm{TG}=\mathrm{G} 2: \mathrm{OG}=\mathrm{G} 1: \mathrm{TS}=\mathrm{S} 1: \mathrm{OS}=\mathrm{S} 4: \mathrm{CS}=\mathrm{S} 1$ & 3.288 \\
21 & 6 & $\mathrm{CC}=\mathrm{C} 1: \mathrm{TG}=\mathrm{G} 2: \mathrm{OG}=\mathrm{G} 1: \mathrm{TS}=\mathrm{S} 3: \mathrm{OS}=\mathrm{S} 2: \mathrm{CS}=\mathrm{S} 3$ & 3.497 \\
22 & 2 & $\mathrm{CC}=\mathrm{C} 1: \mathrm{TG}=\mathrm{G} 2: \mathrm{OG}=\mathrm{G} 1: \mathrm{TS}=\mathrm{S} 1: \mathrm{OS}=\mathrm{S} 2: \mathrm{CS}=\mathrm{S} 3$ & 4.013 \\
23 & 29 & $\mathrm{CC}=\mathrm{C} 1: \mathrm{TG}=\mathrm{G} 4: \mathrm{OG}=\mathrm{G} 3: \mathrm{TS}=\mathrm{S} 3: \mathrm{OS}=\mathrm{S} 2: \mathrm{CS}=\mathrm{S} 1$ & 4.048 \\
24 & 15 & $\mathrm{CC}=\mathrm{C} 1: \mathrm{TG}=\mathrm{G} 2: \mathrm{OG}=\mathrm{G} 3: \mathrm{TS}=\mathrm{S} 3: \mathrm{OS}=\mathrm{S} 4: \mathrm{CS}=\mathrm{S} 1$ & 4.252 \\
25 & 5 & $\mathrm{CC}=\mathrm{C} 1: \mathrm{TG}=\mathrm{G} 2: \mathrm{OG}=\mathrm{G} 1: \mathrm{TS}=\mathrm{S} 3: \mathrm{OS}=\mathrm{S} 2: \mathrm{CS}=\mathrm{S} 1$ & 4.392 \\
26 & 25 & $\mathrm{CC}=\mathrm{C} 1: \mathrm{TG}=\mathrm{G} 4: \mathrm{OG}=\mathrm{G} 3: \mathrm{TS}=\mathrm{S} 1: \mathrm{OS}=\mathrm{S} 2: \mathrm{CS}=\mathrm{S} 1$ & 4.523 \\
27 & 11 & $\mathrm{CC}=\mathrm{C} 1: \mathrm{TG}=\mathrm{G} 2: \mathrm{OG}=\mathrm{G} 3: \mathrm{TS}=\mathrm{S} 1: \mathrm{OS}=\mathrm{S} 4: \mathrm{CS}=\mathrm{S} 1$ & 4.643 \\
28 & 14 & $\mathrm{CC}=\mathrm{C} 1: \mathrm{TG}=\mathrm{G} 2: \mathrm{OG}=\mathrm{G} 3: \mathrm{TS}=\mathrm{S} 3: \mathrm{OS}=\mathrm{S} 2: \mathrm{CS}=\mathrm{S} 3$ & 4.869 \\
29 & 1 & $\mathrm{CC}=\mathrm{C} 1: \mathrm{TG}=\mathrm{G} 2: \mathrm{OG}=\mathrm{G} 1: \mathrm{TS}=\mathrm{S} 1: \mathrm{OS}=\mathrm{S} 2: \mathrm{CS}=\mathrm{S} 1$ & 5.115 \\
30 & 10 & $\mathrm{CC}=\mathrm{C} 1: \mathrm{TG}=\mathrm{G} 2: \mathrm{OG}=\mathrm{G} 3: \mathrm{TS}=\mathrm{S} 1: \mathrm{OS}=\mathrm{S} 2: \mathrm{CS}=\mathrm{S} 3$ & 5.405 \\
31 & 13 & $\mathrm{CC}=\mathrm{C} 1: \mathrm{TG}=\mathrm{G} 2: \mathrm{OG}=\mathrm{G} 3: \mathrm{TS}=\mathrm{S} 3: \mathrm{OS}=\mathrm{S} 2: \mathrm{CS}=\mathrm{S} 1$ & 5.805 \\
32 & 9 & $\mathrm{CC}=\mathrm{C} 1: \mathrm{TG}=\mathrm{G} 2: \mathrm{OG}=\mathrm{G} 3: \mathrm{TS}=\mathrm{S} 1: \mathrm{OS}=\mathrm{S} 2: \mathrm{CS}=\mathrm{S} 1$ & 6.552 \\
\hline
\end{tabular}

Table 5-6: List of all feasible configurations generated by the tool along with their respective penalties

We observe from the result list that, except for components for slot $\mathrm{CC}$, simple components are more suitable for implementation than their concurrent counterparts. This can be attributed to the fact that tasks are not saturated and hence overhead in concurrency usually degrades performance rather than enhancing it. This, however, is not true for components for slots $\mathrm{CC}$ where the concurrent component $\mathrm{C} 2$ is more suitable than the simple component $\mathrm{C} 1$.

This ranking provides us insight into the suitability of implementations from a performance point of view before actually starting to implement the system. 


\section{Chapter 6: Conclusions}

\subsection{Conclusion to the Work}

This thesis describes a systematic approach to building of performance models of new services and evaluation of them against the requirements. The method could be used to create a new product configuration, or to restructure a system to adapt to a change in workload and enable flexible rapid assembly of components to provide services with required performance characteristics.

A framework for modeling is introduced which involves a high level template representing the basic outline of the system (Section 3.3.1) which contains customized functionalities and slots where other off-the shelf components can be placed. Service types, introduced in Section 3.3.2, constrain the composition of alternatives and isolate the potential complexity introduced by compatibility of components. The concept of groups is introduced in Section 3.3.3 which classifies a component into broad categories based on functionality and it reduces the number of alternatives to be evaluated. It is assumed that the software generation process is sufficiently parameterized, so the detailed functional and interface constraints can be met, if the broad categories are satisfied.

The approach described above has several strengths. It can scale up to large systems with many components using the scalable capabilities of layered queuing analysis and CBML. The modeling environment expresses interface constraints and functionality of 
components abstractly, by typing the ports of the components and by assigning groups of components by functional concerns. Components can be both complex systems with internal concurrency or smaller non-concurrent objects or plug-ins and various extensions to them are trivial. They may be partly defined by parameter values, for example defining the number of replicas of a server or the size of a cache; and these can be treated as distinct component alternatives. Execution environment alternatives (such as hardware platform or choice of middleware) as well as software component choices can be compared.

A method for evaluating suitable alternatives from a performance point of view is presented (Section 3.4). The method allows both strict requirements (in form of hard constrains as described in section 3.4.1.1) and preferences (in form of soft constrains as described in section 3.4.1.2) to be expressed and balanced using prices for soft constraints. It deals with both the kind of preferences efficiently in a separate yet in a unified manner (Section 3.4.2).

As a part of this work, a tool has been designed and implemented. This tool can create and maintain a library of components (Chapter 4). It can do operations like load components and templates, assign groups and edit services. It can build systems based on compatible interfaces and enumerate alternatives. An algorithm for automatic assembly of systems for enumeration based on satisfaction of functional is developed (Section 4.3.3). 
A case study based loosely on an industrial product, The Distributed Telephone Switch demonstrates the full process from capturing requirements to developing and evaluating alternatives (Chapter 5). It justifies the application of the ideas described in thesis (Section 5.1.1). It shows that some parts of the switch require concurrency while others don't. (Section 5.4).

\subsection{Limitations}

The approach creates all feasible alternatives for the system. The major limitation of the approach is that it is not applicable in cases where there is an explosion of alternatives. Explosion of alternatives needs to be controlled by user though the use of groups and services. Moreover it is not possible to enumerate alternatives based on variations of parameters for a component.

Further, in some problem scenarios, it might be difficult to formulate constraint as a linear combination of various performance measures, in which case they may need to be estimated or approximated. In this case, if the sensitivity of these values on performance properties of system is high, the results we obtain might be misleading.

The Library Manager tool developed as part of the work can enumerate all the possible alternatives of the system. However the evaluation of alternatives still is a tedious job that is not handled by the tool. 


\subsection{Future Research}

The future work could investigate evaluation of alternatives for non-linear constraints. Another research direction would be to expanding the scope of the approach to include methods for enumerating alternatives based on variation of parameters. Also, libraries of commonly used components could be developed to facilitate further research. Given the amount of resources this method saves, this field, once completely researched and investigated, could affect industrial software development process in a fundamental way. 


\section{$\underline{\text { References }}$}

1. F. Bause and P. S. Kritzinger. "Stochastic Petri nets: an introduction to the theory", Wiesbaden, Vieweg Verlag, Germany, 2002

2. J. Bosch. "Design and use of software architectures; Adopting and evolving a product-line approach", Addison-Wesley, 2000

3. X. Cai, M. R. Lyu, K. F. Wong, R. Ko, "Component-based software engineering: technologies, development frameworks, and quality assurance schemes", Proceedings of APSEC 2000: Software Engineering Conference, 2000. Seventh Asia-Pacific, 5-8 Dec. 2000 , pp $372-379$

4. S. Chen, I. Gorton, A. Liu, Y. Liu, "Performance Prediction of COTS Componentbased Enterprise Applications", Proceedings of 5th ICSE Workshop on ComponentBased Software Engineering: Benchmarks for Predictable Assembly, http://www.sei.cmu.edu/pacc/CBSE5/liu-cbse5-29.pdf, Orlando, Florida USA, May $19-20,2002$

5. Mike Clark, Peter Fletcher, J. Jeffrey Hanson, Romin Irani, Mark Waterhouse, and Jorgen Thelin, "Web Services Business Strategies and Architectures", Expert Press, August 2002

6. P. Clements and L. Northrop. "Software Product Lines; Practices and Patterns", Addison-Wesley, 2000 
7. Canadian Radio and Telecommunications Commission, "Telecom decision 97-16: Quality of service indicators for use in telephone company regulation", July 1997

8. K. Czarnecki and U. W. Eisenecker, "Generative Programming", Addison-Wesley, 2001

9. R.G. Franks, C. M. Woodside, "Effectiveness of Early Replies in Client-Server Systems", Performance Evaluation, v 36-37, pp 165 - 184, 1999

10. G. Franks, M. Woodside, "Performance of Multi-level Client-Server Systems with Parallel Service Operations", Proc. First Int. Workshop on Software and Performance (WOSP98), pp. 120-130, Santa Fe, October 1998

11. G. Franks, S. Majumdar, J. Neilson, D. Petriu, J. Rolia, and M. Woodside, "Performance Analysis of Distributed Server Systems," in The Sixth International Conference on Software Quality (6ICSQ), Ottawa, Ontario, 1996, pp. 15-26

12. G.T. Heineman, W. T. Councill, "Component-Based Software Engineering; Putting the Pieces Together ", Addison-Wesley, 2001

13. International Telecommunication Union, "ITU recommendation H.248: Gateway control protocol", March 2002

14. P. Jogalekar and C.M. Woodside, "Evaluating the Scalability of Distributed Systems", Proc. of Hawaii Int. Conference on System Sciences, January 1998

15. J. P. Lopez-Grao, J. Merseguer, J. Campos, "From UML Activity Diagrams To Stochastic Petri Nets: Application To Software Performance Engineering", Proc. Forth Int. Workshop on Software and Performance (WOSP'04), pp. 25-36, Redwood Shores, January 2004 
16. J. Luthi. "Interval matrices for the bottleneck analysis of queuing network models with histogram-based parameters", Proceedings of International Computer Performance and Dependability Symposium (IPDS), 7-9 Sept. 1998, pp142-151

17. S. Majumdar and T. Phillips. "Performance of scheduling strategies for client-server systems", Proceedings of International Conference on Parallel and Distributed Systems, 3-6 June 1996. pp 448-455

18. D. A. Menasce. "Two-Level Iterative Queuing Modeling of Software Contention", Proceedings of MASCOTS 2002, October, Fort Worth, Texas, USA. pp267-280

19. J.E. Neilson, C.M. Woodside, D.C Petriu and S. Majumdar, "Software Bottlnecking in Client-Server Systems and Rendezvous Networks", IEEE Trans. On Software Engineering, Vol. 21, No. 9, pp. 776-782, September 1995

20. J.Q. Ning, "Component-based software engineering (CBSE)", Proceedings of the Fifth International Symposium on Assessment of Software Tools and Technologies, 1997, 2-5 June 1997 pp $34-43$

21. Object Management Group, "UML 2", ptc/03-08-02, August 2, 2003 (Accessible from http://www.omg.org/docs/ptc/03-08-02.pdf)

22. J.A. Rolia and K.C. Sevcik, "The Method of Layers", IEEE Transactions on Software Engineering, Vol. 21, No. 8 , Aug. 1995, pp $689-700$

23. F. Sheikh and C.M. Woodside, "Layered Analytic Performance Modelling of a Distributed Database System", Proc. 1997 International Conf. on Distributed Computing Systems, May 1997, pp. 482-490 
24. Richard Singer, "Overview of 5ESS R-2000 switch performance," in Proceeding of the First International Workshop on Software and Performance, WOSP'98, Santa Fe, New Mexico, USA, Oct. 1998, pp. 7 - 13

25. M. Sitaraman, G. Kulczycki, J. Krone, W. F. Ogden and A.L.N. Reddy "Performance Specification of Software Components", Proceedings of SSR '01, pp. 3-10. ACM/SIGSOFT, May 2001

26. C. U. Smith, L. G. Williams, "Performance Engineering Evaluation of ObjectOriented Systems with SPE.ED", Lecture Notes in Computer Science 1245: Computer Performance Evaluation Modeling Techniques and Tools, Springer, 1997

27. Connie U. Smith, "Performance of Software Systems", Addison-Wesley, 1990

28. C. Szyperski, "Component Software; Beyond Object-Oriented Programming", Addison-Wesley, 1998

29. Telcordia Technologies, "LSSGR: service standards", Generic Requirement GR-511CORE, June 2000

30. Telcordia Technologies, "LSSGR: traffic capacity and environment", Generic Requirement GR-517-CORE, December 1998

31. Telcordia Technologies, "Voice over packet in next generation networks: An architectural framework", Special Report SR-4717, January 1999

32. Peter Tregunno, "Practical Analysis of Software Bottlenecks", M.A.Sc thesis, Carleton University, May 2003.

33. C.M. Woodside, J.E. Neilson, D.C. Petriu, S. Majumdar, "The Stochastic Rendezvous Network Model for Performance of Synchronous Client-Server-like Distributed Software", IEEE Transactions on Computers, Vol.44, Nb.1, pp 20-34, January 1995 
34. M. Woodside "Scalability metrics and analysis of mobile agent systems", Proc. Workshop on Infrastructure for Scalable Mobile Agent Systems, at Autonomous Agents 2000, Barcelona; June 3, 2000

35. C.M. Woodside, "Throughput Calculation for Basic Stochastic Rendezvous Networks", Performance Evaluation, Vol. 9, No. 2, April 1989, pp143-160

36. Xiuping $\mathrm{Wu}, "$ An Approach to Predicting Performance for Component Based Systems", M.A.Sc thesis, Carleton University, July 2003

37. X. Wu and M. Woodside, "Performance Modeling from Software Components", Proc. Forth Int. Workshop on Software and Performance (WOSP’04), pp. 290-301, Redwood Shores, January 2004

38. World Wide Web Consortium, "Extensible Markup Language (XML) 1.0 (Third Edition)", Feb 04, 2004; http://www.w3.org/TR/2004/REC-xml-20040204/

39. R. A. Wyke and A. Watt. "XML Schema Essentials", Wiley Computer Publishing, 2002 


\section{$\underline{\text { Appendix A }}$}

\section{Diagrams for components used in example of Chapter 3}

\section{A.1 Component M1 for template of Figure 3-7}

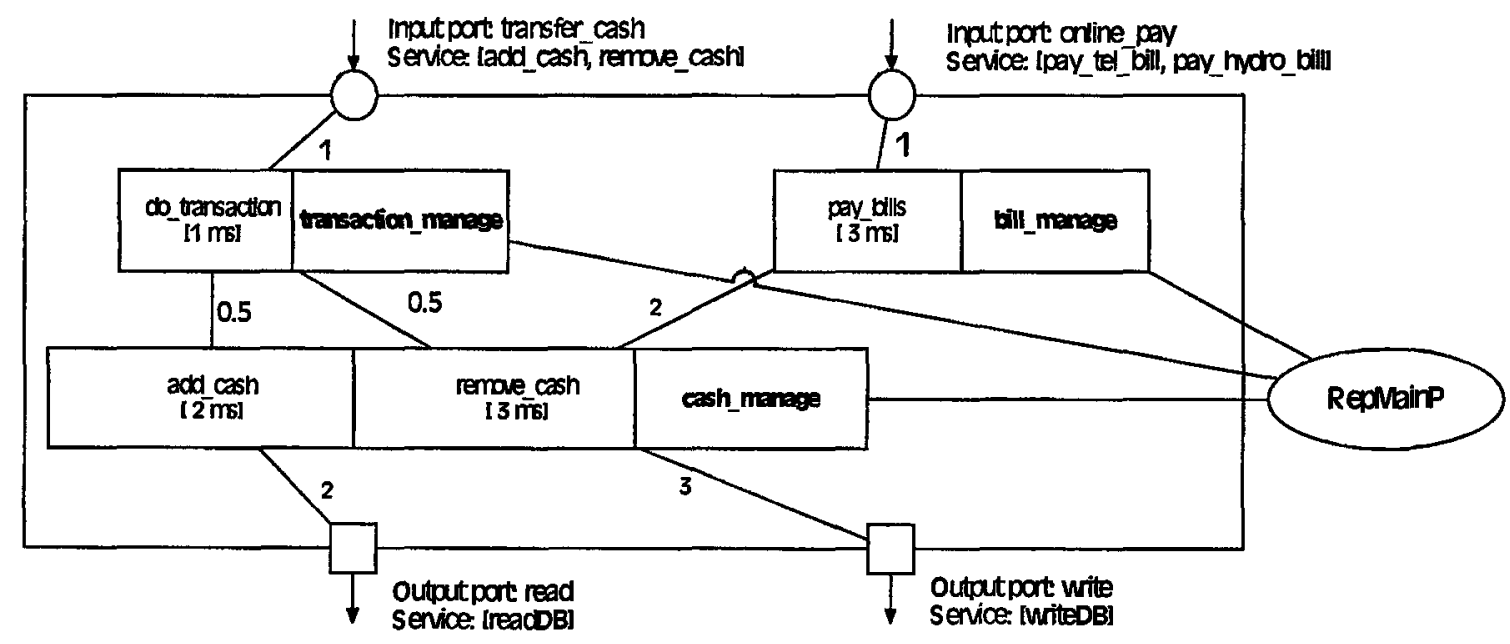

\section{A.2 Component M2 for template of Figure 3-7}

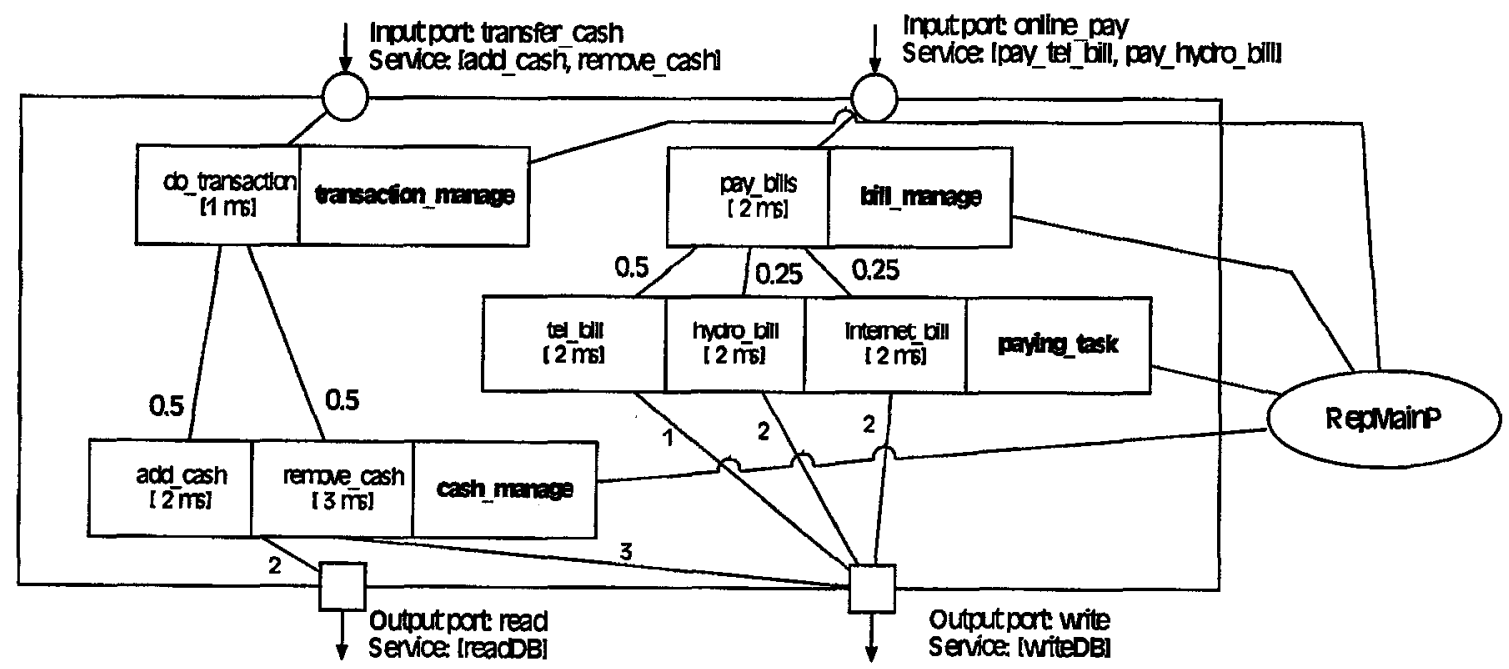




\section{A.3 Component D1 for template of Figure 3-7}

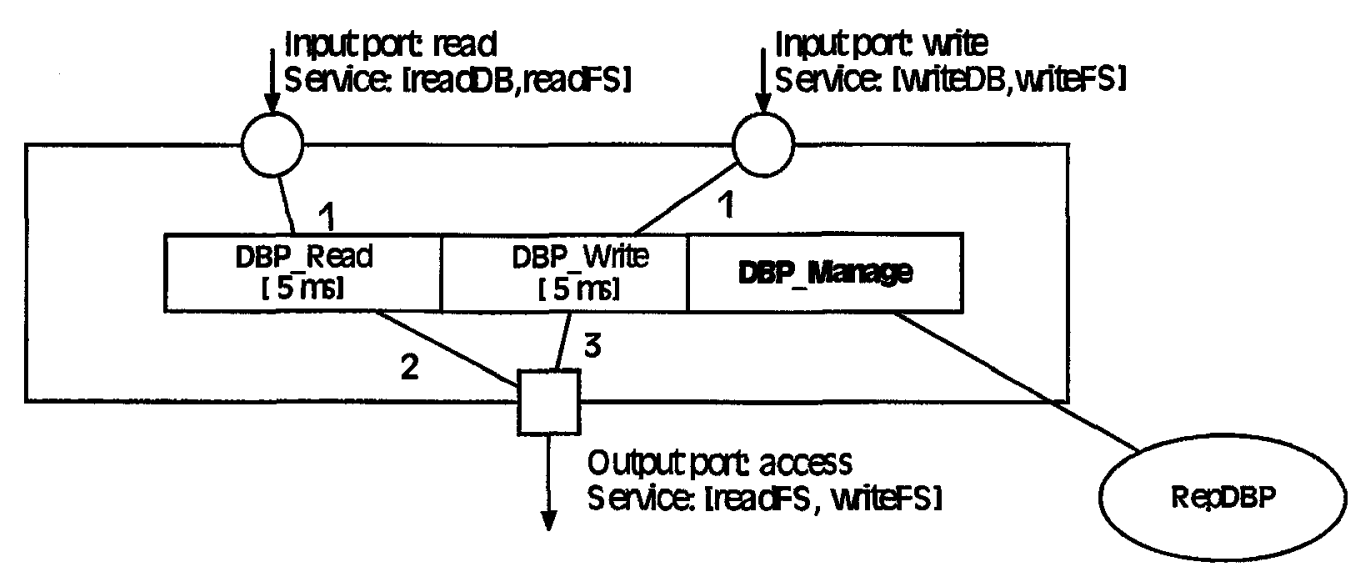

\section{A.4 Component D2 for template of Figure 3-7}

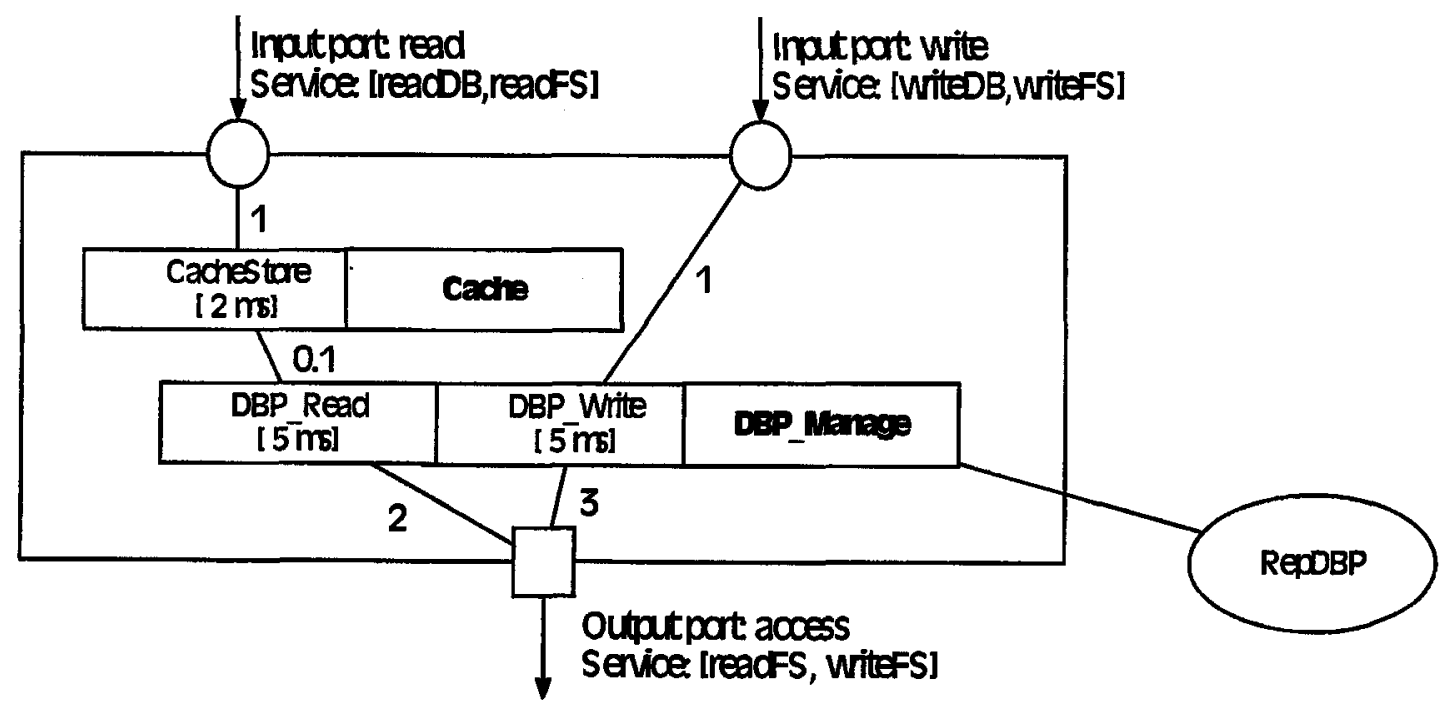




\section{A.5 Component D3 for template of Figure 3-7}

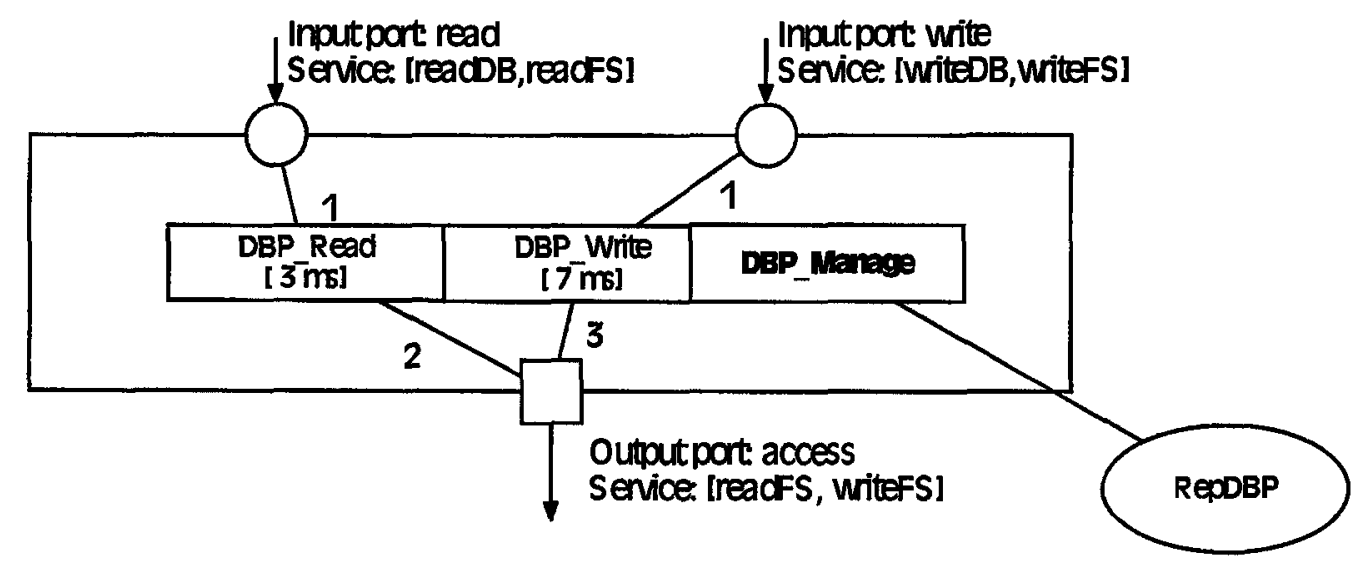

A.6 Component F for template of Figure 3-7

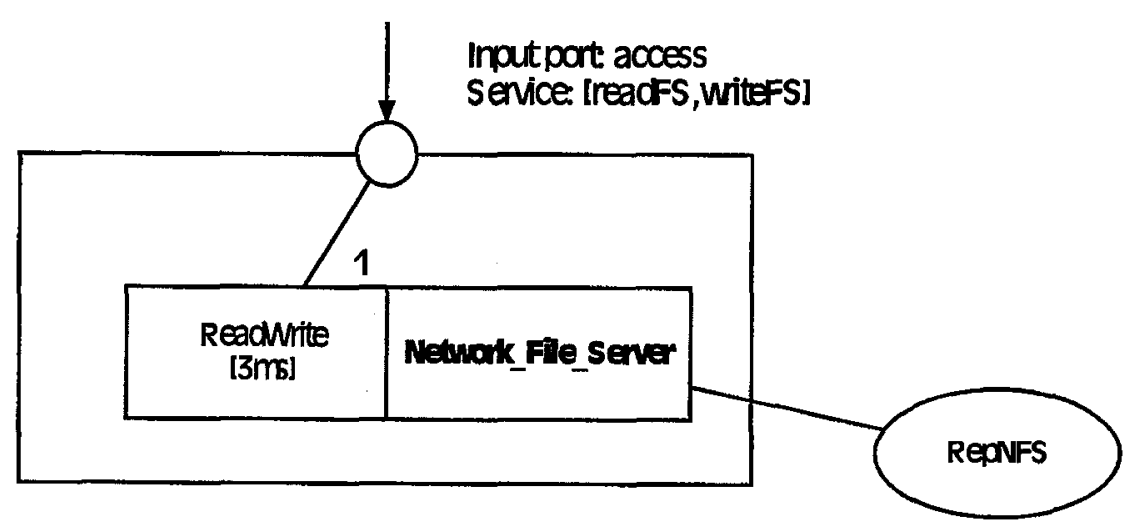




\section{Appendix B \\ CBML code for components used in case study of Chapter 5}






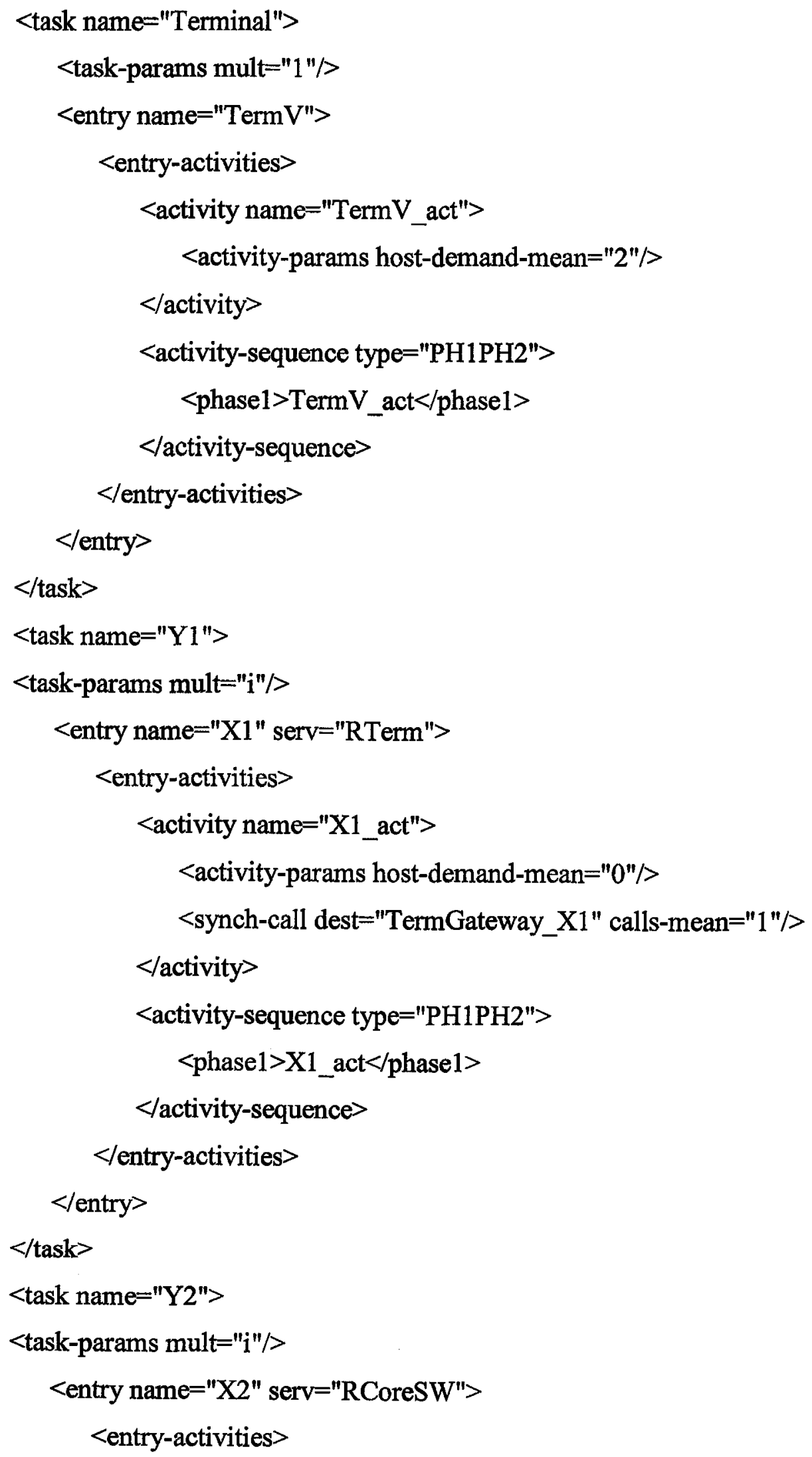




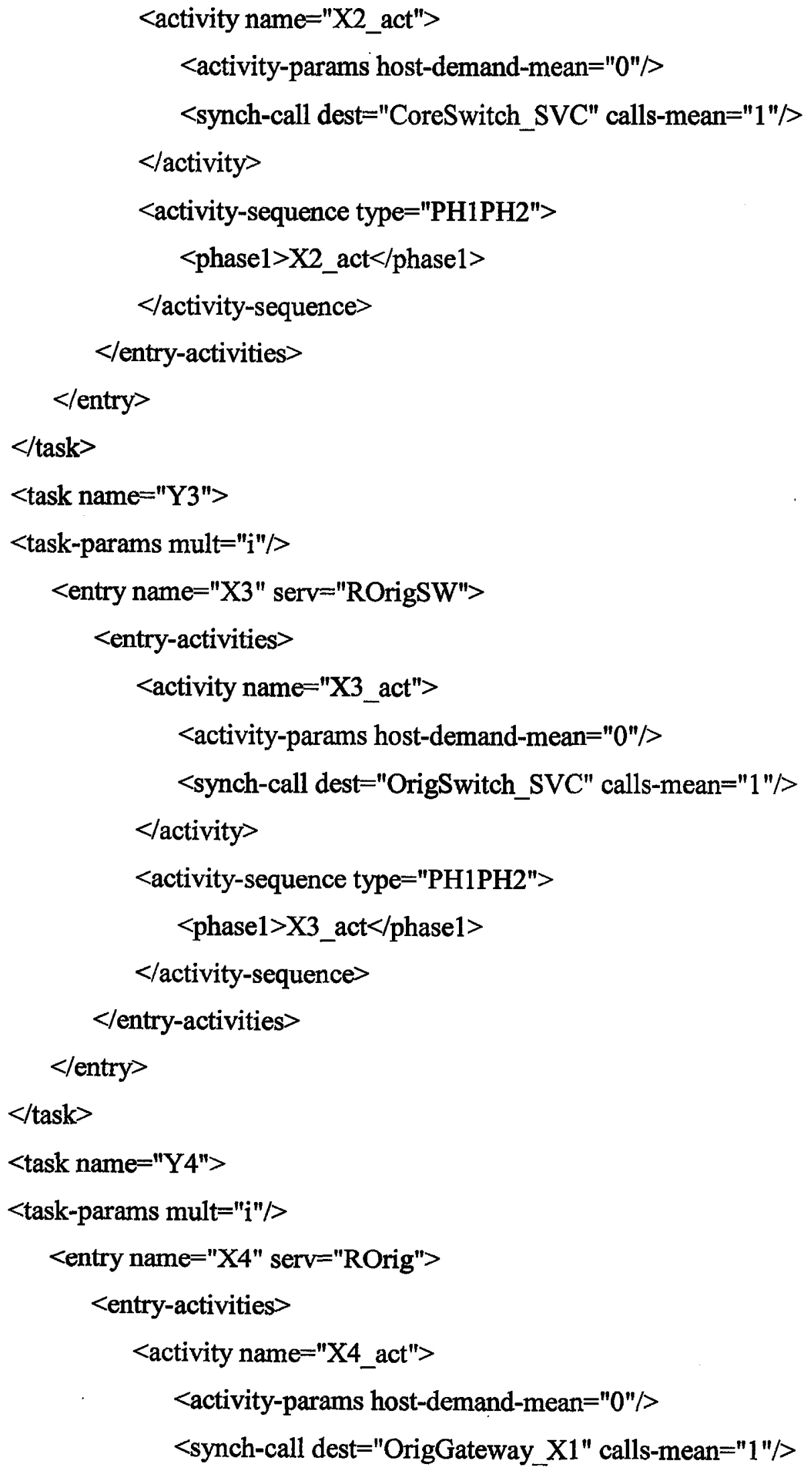




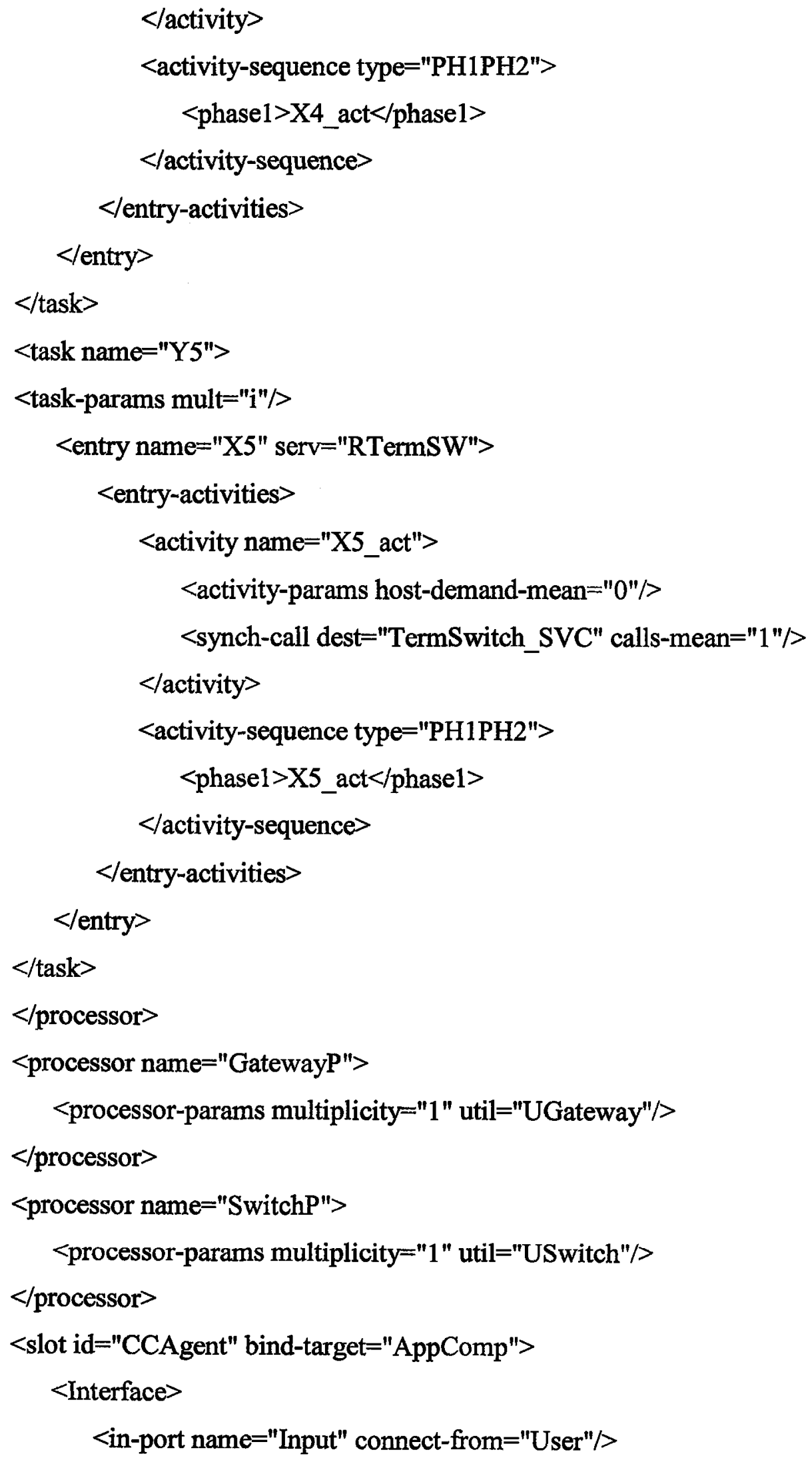




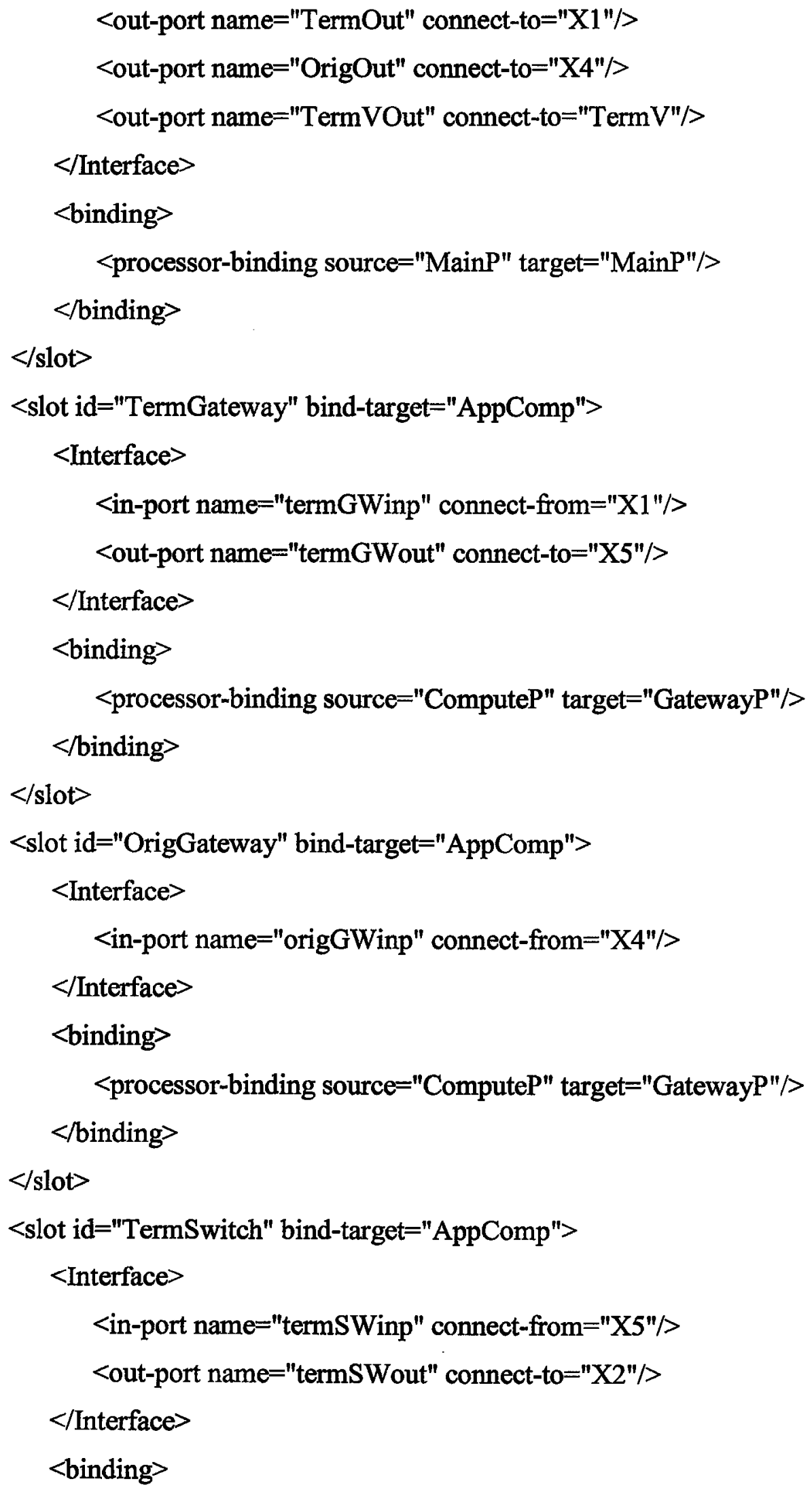




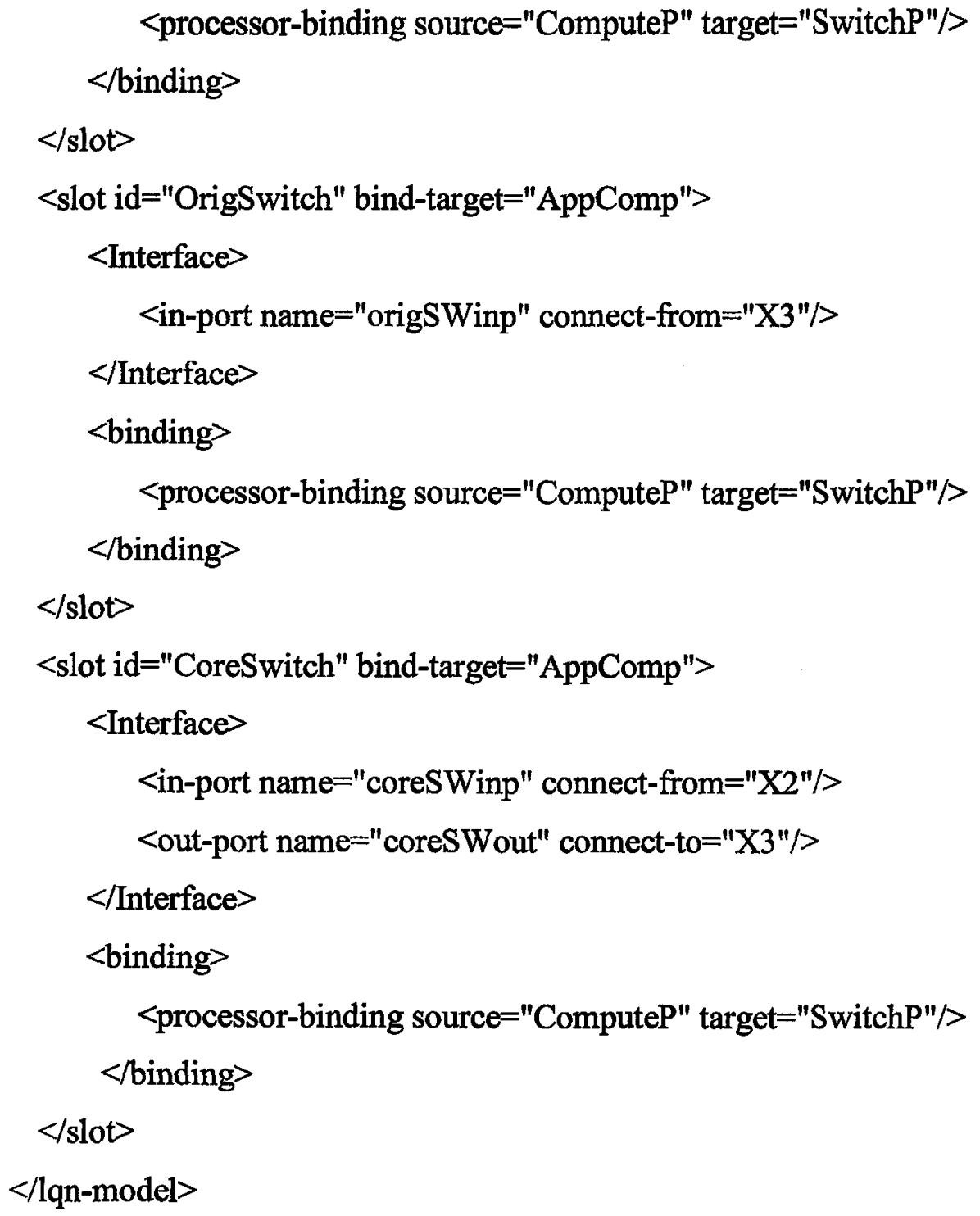

\section{B.2 Component G1 at Figure 5-2}

<lqn-submodel name="Gateway1" description="A submodel for Application Server in XML" xmlns:xsi="http://www.w3.org/2001/XMLSchema-instance"

xsi:noNamespaceSchemaLocation="lqn-sub.xsd">

$<$ Interface $>$

$<$ in-port name="gatewayInp" connect-to="X1" description="Reporting request controller"/>

$<$ Replaceable-Processor name="ComputeP"/>

$</$ Interface $>$ 


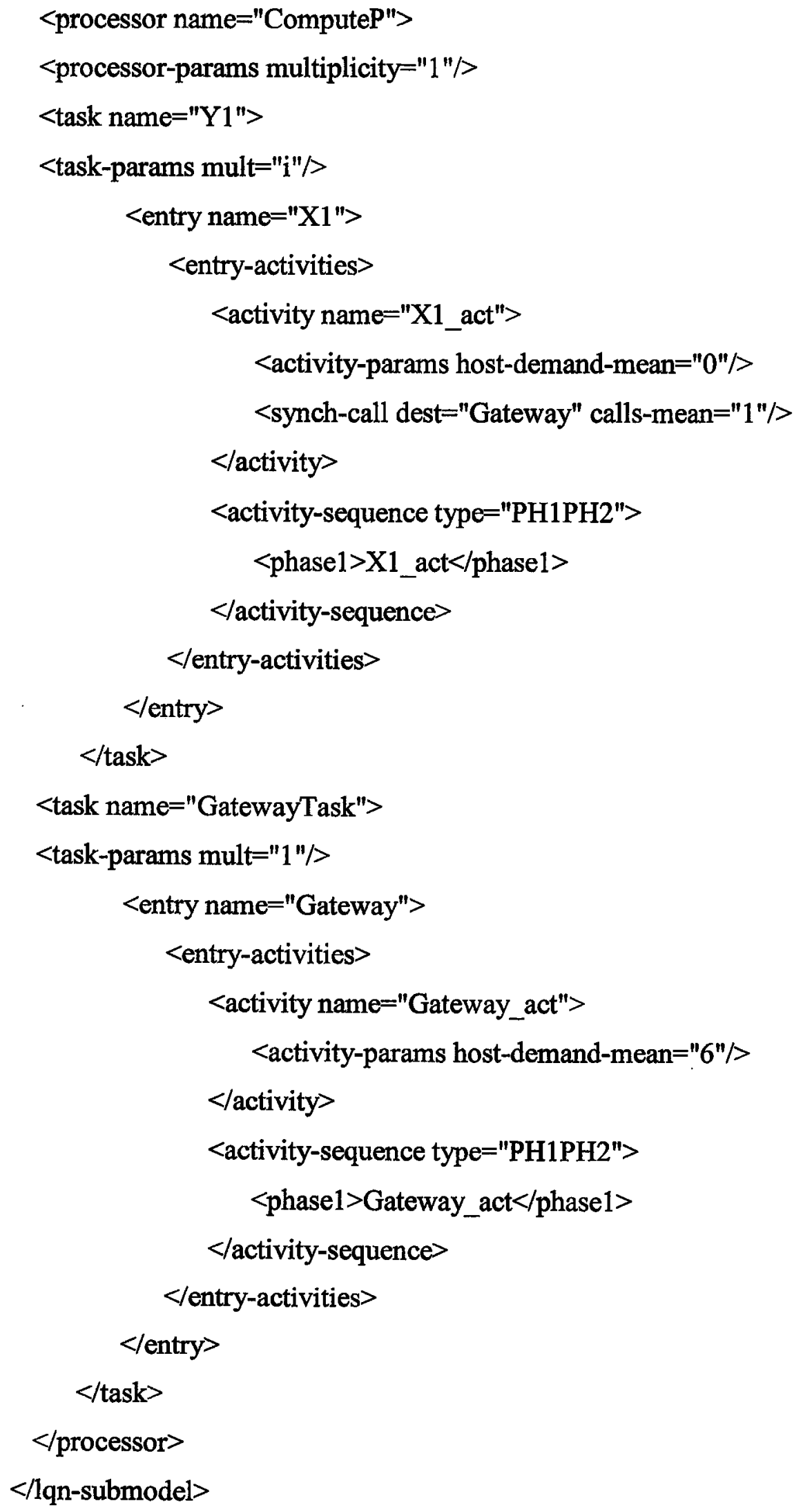




\section{B.3 Component G2 at Figure 5-3}

$<$ lqn-submodel name="Gateway2" description="A submodel for Application Server in XML" xmlns:xsi="http://www.w3.org/2001/XMLSchema-instance"

xsi:noNamespaceSchemaLocation="lqn-sub.xsd">

$<$ Interface $>$

$<$ in-port name="gatewayInp" connect-to="X1" description="Reporting request controller"/>

$<$ out-port name="gatewayOut" connect-from="X2" description="big report request"/>

$<$ Replaceable-Processor name="ComputeP"/ $>$

$</$ Interface $>$

$<$ processor name="ComputeP">

$<$ processor-params multiplicity="1"/>

$<$ task name="Y1">

$<$ task-params mult="i" $>$

$<$ entry name $=" \mathrm{X} 1 ">$

$<$ entry-activities $>$

<activity name="X1_act">

$<$ activity-params host-demand-mean="0"/>

$<$ synch-call dest="Gateway" calls-mean="1"/>

$</$ activity $>$

$<$ activity-sequence type="PH1PH2">

$<$ phase1 $>$ X1_act $</$ phase $1>$

$</$ activity-sequence $>$

$<$ entry-activities $>$

$<$ entry $>$

$<$ task $>$

$<$ task name $=" Y 2 ">$

$<$ task-params mult="i">

$<$ entry name $=$ "X2">

$<$ entry-activities> 


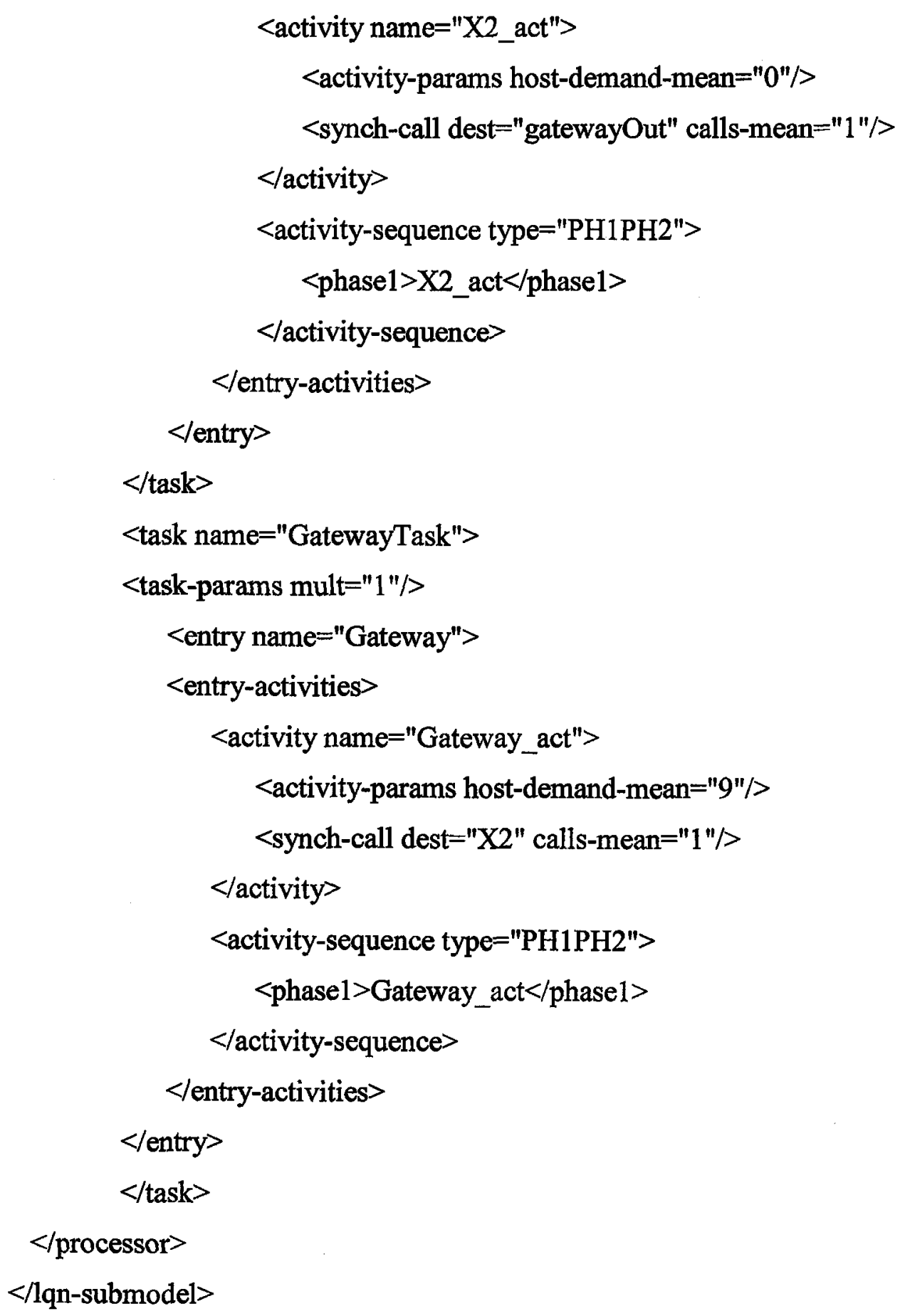

\section{B.4 Component G3 at Figure 5-4}

$<$ lqn-submodel name="Gateway3" description="A submodel for Application Server in XML" xmlns:xsi="http://www.w3.org/2001/XMLSchema-instance" xsi:noNamespaceSchemaLocation="lqn-sub.xsd"> 


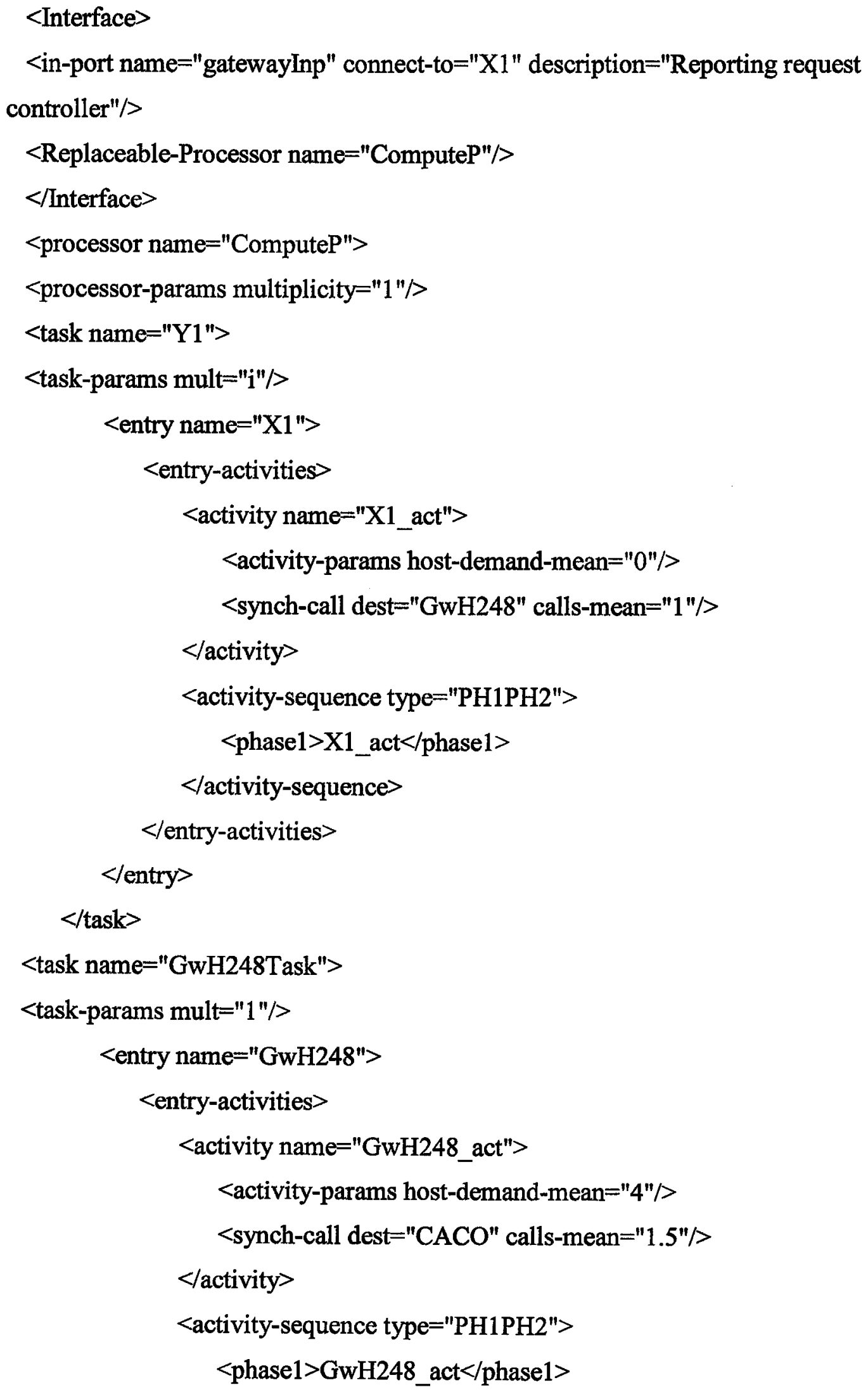




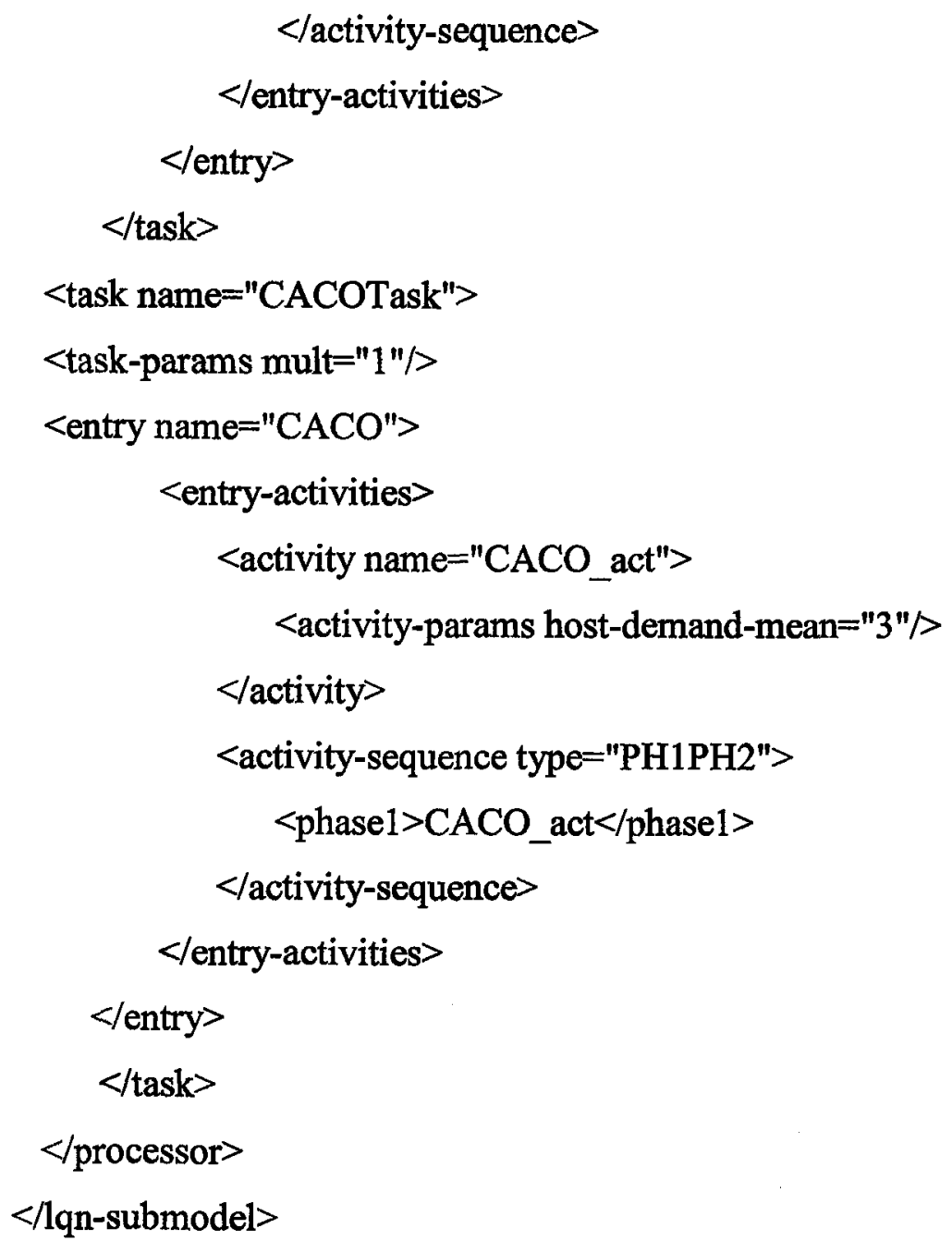

\section{B.5 Component G4 at Figure 5-5}

$<$ lqn-submodel name="Gateway4" description="A submodel for Application Server in XML" xmlns:xsi="http://www.w3.org/2001/XMLSchema-instance" xsi:noNamespaceSchemaLocation="lqn-sub.xsd">

$<$ Interface $>$

$<$ in-port name="gatewayInp" connect-to="X1" description="Reporting request controller"/>

$<$ out-port name="gatewayOut" connect-from="X2" description="big report request" $>$

$<$ Replaceable-Processor name="ComputeP"/>

$</$ Interface $>$

$<$ processor name="ComputeP"> 


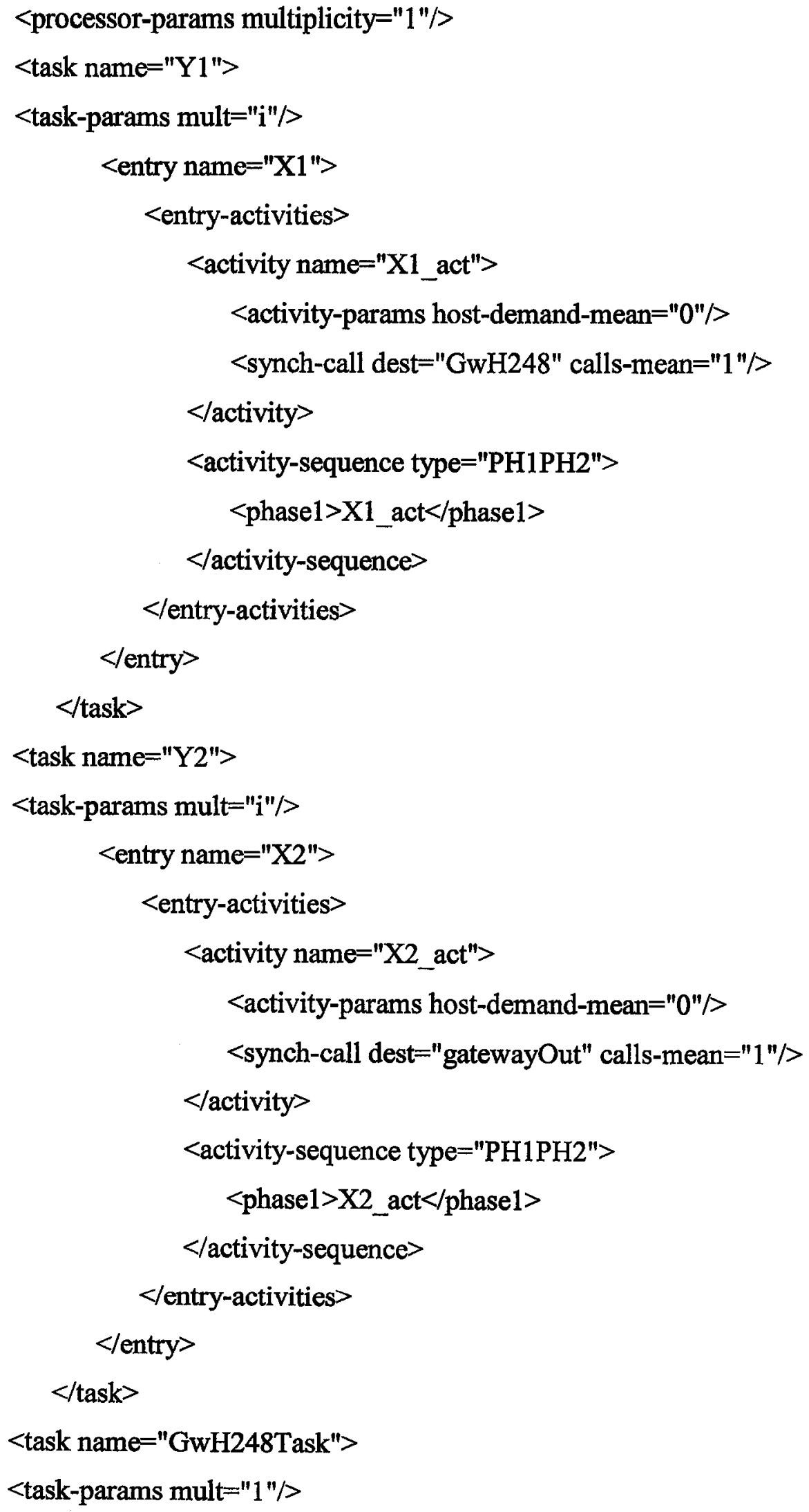




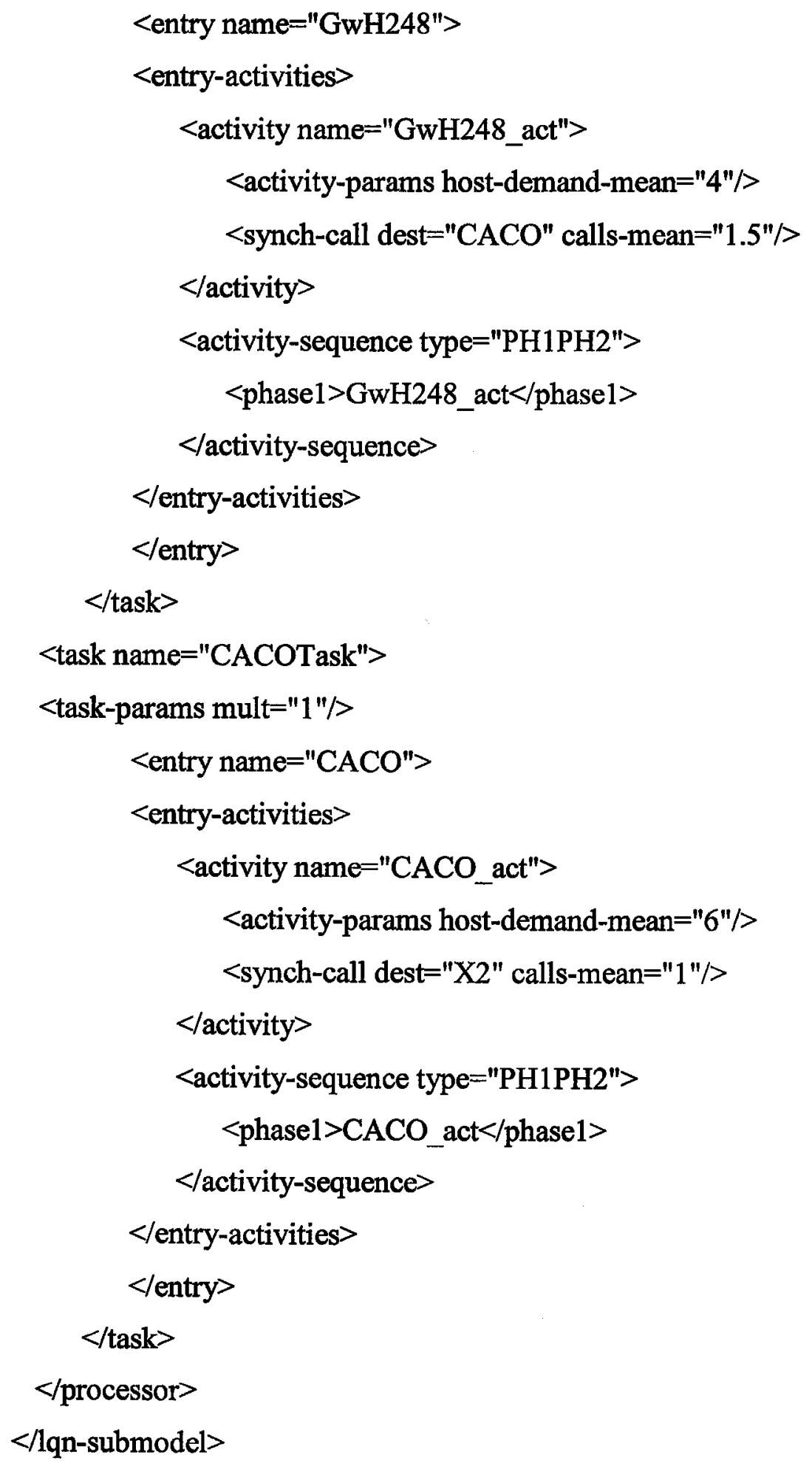

\section{B.6 Component S1 at Figure 5-7}


<lqn-submodel name="Switch2" description="A submodel for Application Server in XML" xmlns:xsi="http://www.w3.org/2001/XMLSchema-instance" xsi:noNamespaceSchemaLocation="lqn-sub.xsd">

<Interface>

<in-port name="switch_input" connect-to="SVC" description="Reporting request controller"/>

$<$ Replaceable-Processor name="ComputeP" $>$

$</$ Interface $>$

<processor name="ComputeP">

<processor-params multiplicity="1"/>

<task name="SwitchTask">

$<$ task-params mult="1"/>

<entry name $=$ "SVC">

$<$ entry-activities>

<activity name="SVC_act">

<activity-params host-demand-mean="1.5"/>

$<$ synch-call dest="CTL" calls-mean="5"/>

$<$ synch-call dest="NLC" calls-mean="4"/>

$<$ synch-call dest="ILC" calls-mean="7"/>

$</$ activity $>$

<activity-sequence type="PH1PH2">

$<$ phase1 $>$ SVC_act $</$ phase1 $>$

$<$ /activity-sequence $>$

$</$ entry-activities $>$

$<$ entry $>$

$<$ task $>$

<task name="MainTask">

<task-params mult="1"/>

<entry name="CTL">

<entry-activities>

<activity name="CTL_act"> 


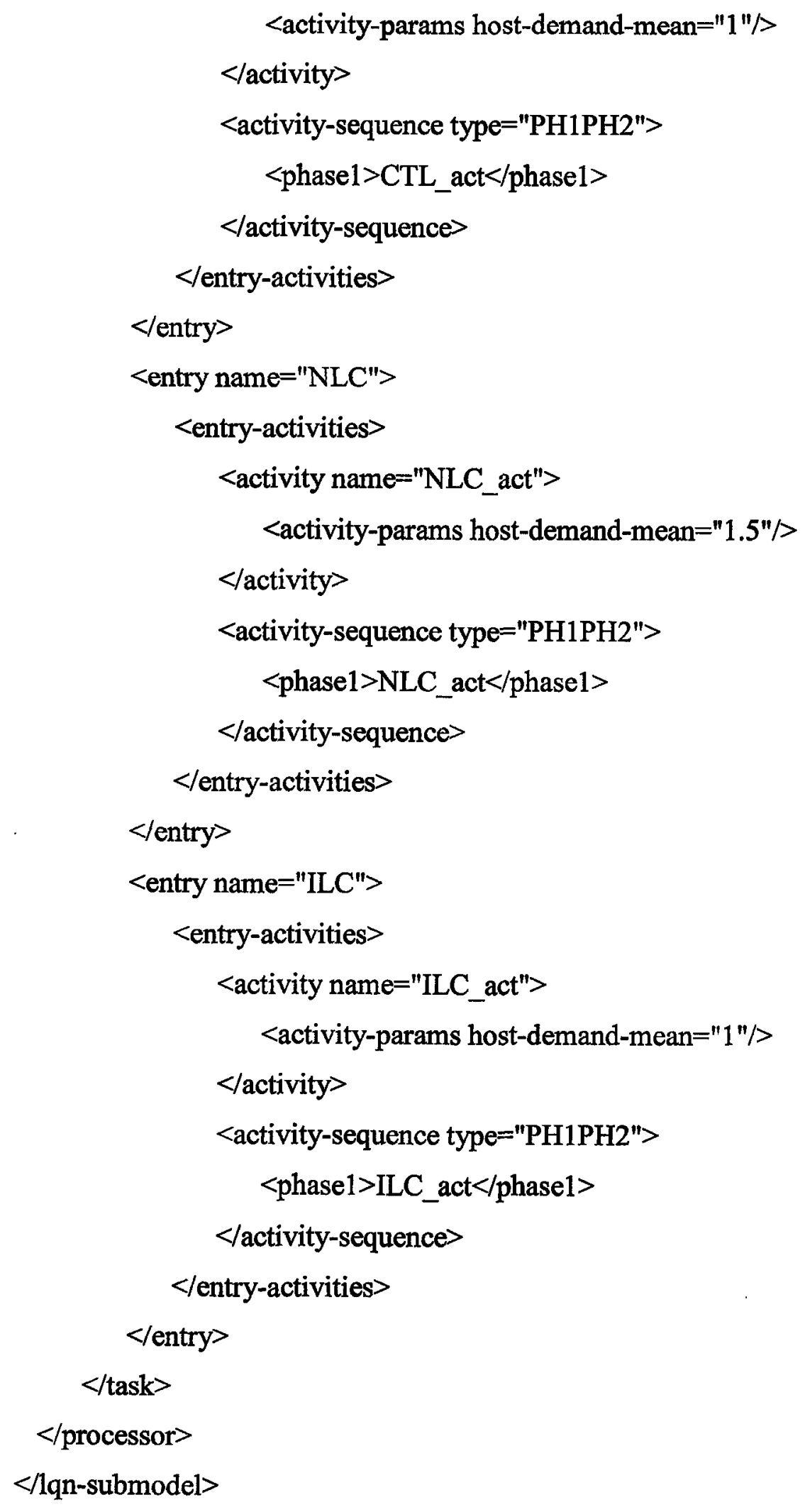




\section{B.7 Component S2 at Figure 5-8}

$<$ lqn-submodel name="Switch1" description="A submodel for Application Server in XML" xmlns:xsi="http://www.w3.org/2001/XMLSchema-instance"

xsi:noNamespaceSchemaLocation="lqn-sub.xsd">

$<$ Interface $>$

$<$ in-port name="switch_input" connect-to="SVC" description="Reporting request controller"/>

$<$ out-port name="switch_output" connect-from="SVC" description="big report request"/>

$<$ Replaceable-Processor name="ComputeP"/ $>$

$</$ Interface $>$

$<$ processor name="ComputeP">

$<$ processor-params multiplicity="1"/>

$<$ task name="SwitchTask" $>$

$<$ task-params mult="1">>

$<$ entry name $="$ SVC">

$<$ entry-activities>

$<$ activity name="SVC_act">

$<$ activity-params host-demand-mean="2"/>

$<$ synch-call dest="switch_output" calls-mean="2"/>

$<$ synch-call dest="CTL" calls-mean="5"/>

$<$ synch-call dest $=" N L C "$ calls-mean="4" $>>$

$<$ synch-call dest="ILC" calls-mean="7"/>

$<$ activity $>$

$<$ activity-sequence type="PH1PH2">

$<$ phase1 $>$ SVC_act $<$ phase $1>$

$<$ activity-sequence $>$

$<$ entry-activities $>$

$<$ entry $>$

$<$ task $>$

$<$ task name="MainTask"> 


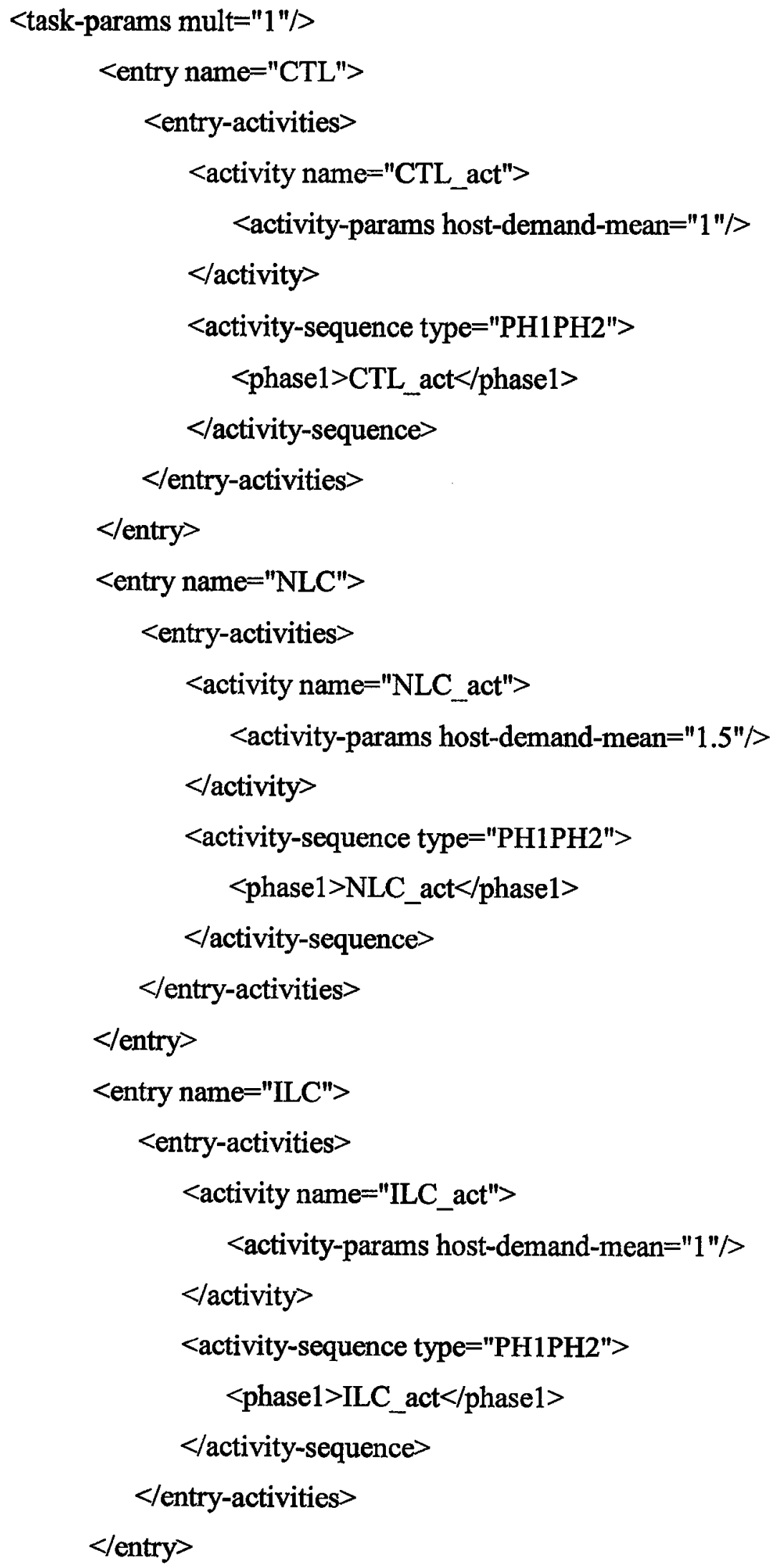




$$
\begin{gathered}
</ \text { task }> \\
</ \text { processor }> \\
</ \text { lqn-submodel }>
\end{gathered}
$$

\section{B.8 Component S3 at Figure 5-9}

<lqn-submodel name="Switch4" description="A submodel for Application Server in XML" xmlns:xsi="http://www.w3.org/2001/XMLSchema-instance"

xsi:noNamespaceSchemaLocation="lqn-sub.xsd">

$<$ Interface $>$

<in-port name="switch_input" connect-to="SVC" description="Reporting request controller"/>

$<$ Replaceable-Processor name="ComputeP" $>$

$</$ Interface $>$

$<$ processor name $=$ "ComputeP">

$<$ processor-params multiplicity="1"/>

$<$ task name="SwitchTask" $>$

<task-params mult="1"/>

$$
\begin{aligned}
& <\text { entry name="SVC"> } \\
& <\text { entry-activities }> \\
& <\text { activity name="SVC_act"> } \\
& <\text { activity-params host-demand-mean="1.5"/> } \\
& <\text { synch-call dest="CTL" calls-mean="5"/> } \\
& <\text { synch-call dest="NLC" calls-mean="4"/> } \\
& <\text { synch-call dest="ILC" calls-mean="7"/> } \\
& </ \text { activity }> \\
& <\text { activity-sequence type="PH1PH2"> } \\
& <\text { phase1>SVC_act</phase1> } \\
& </ \text { activity-sequence }> \\
& </ \text { entry-activities }> \\
& </ \text { entry }>\quad \\
& </ \text { task }>\quad
\end{aligned}
$$




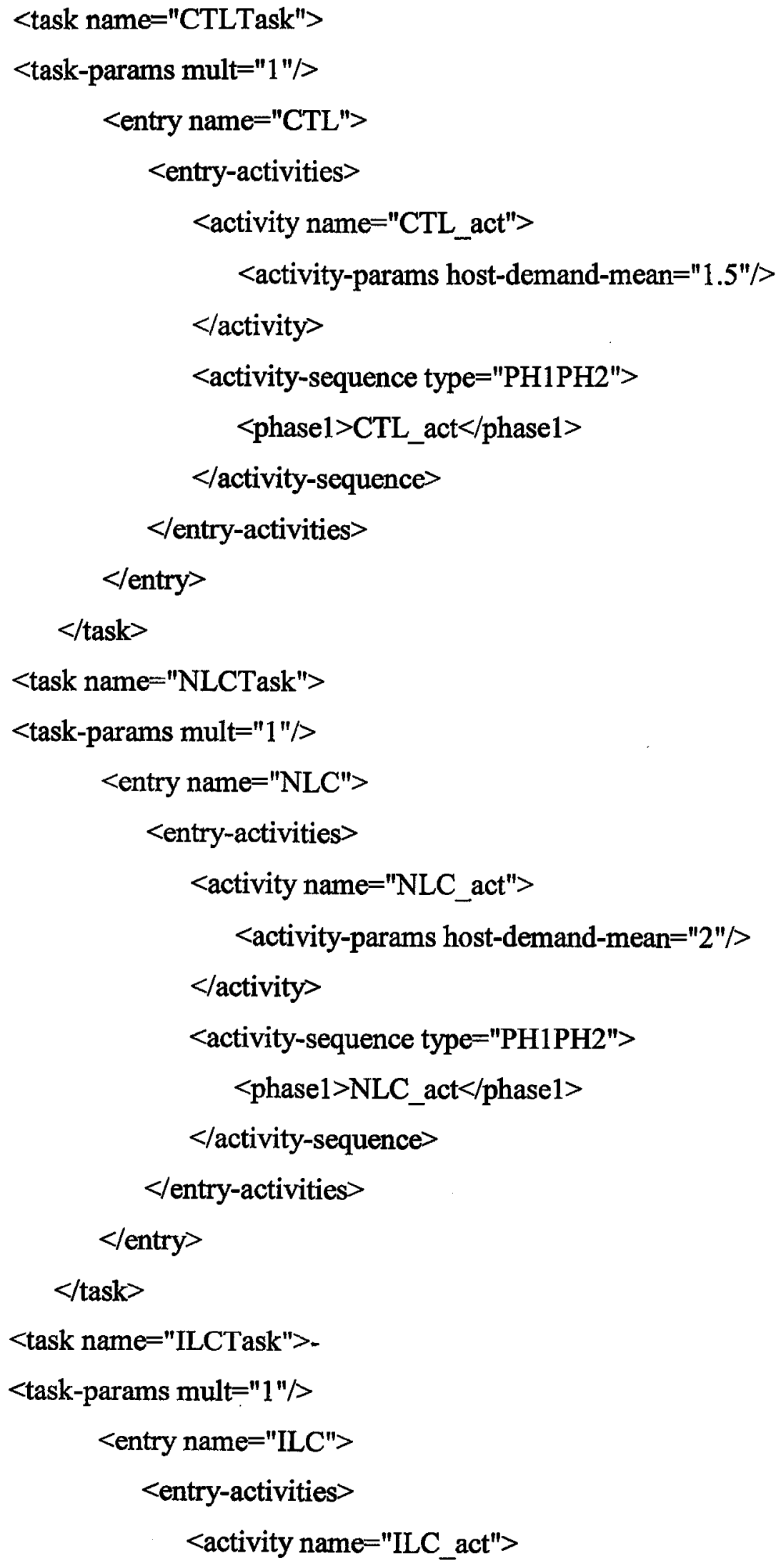




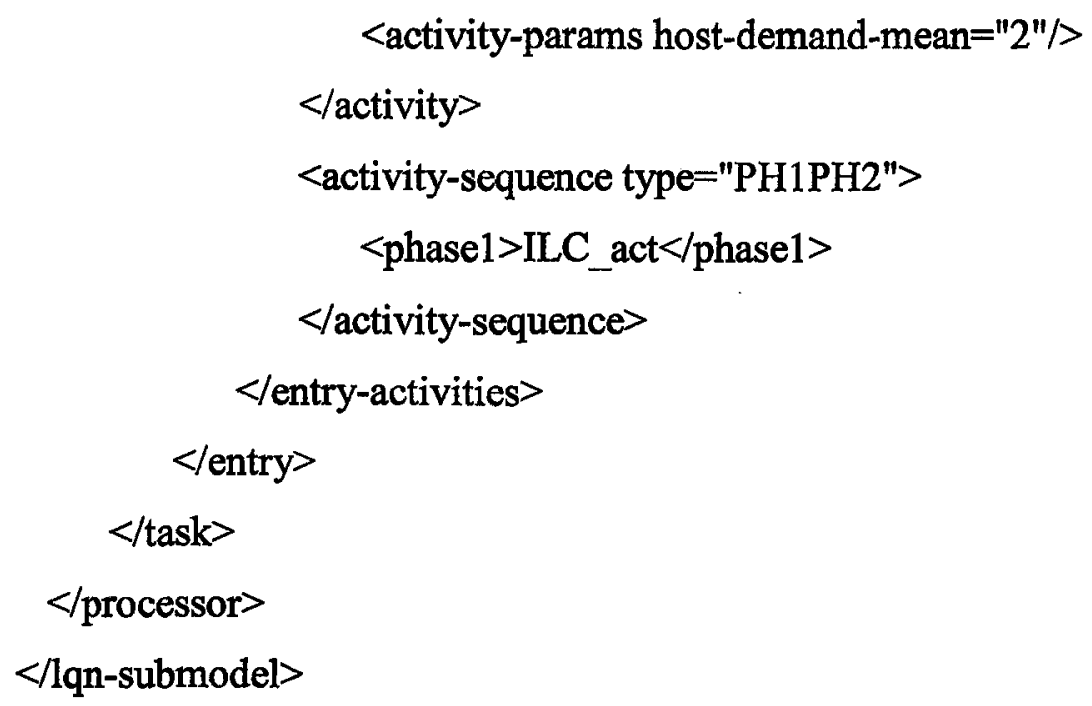

\section{B.9 Component S4 at Figure 5-10}

$<$ lqn-submodel name="Switch3" description="A submodel for Application Server in XML" xmlns:xsi="http://www.w3.org/2001/XMLSchema-instance"

xsi:noNamespaceSchemaLocation="Iqn-sub.xsd">

$<$ Interface $>$

$<$ in-port name="switch_input" connect-to="SVC" description="Reporting request controller"/>

$<$ out-port name="switch_output" connect-from="SVC" description="Reporting request controller"/>

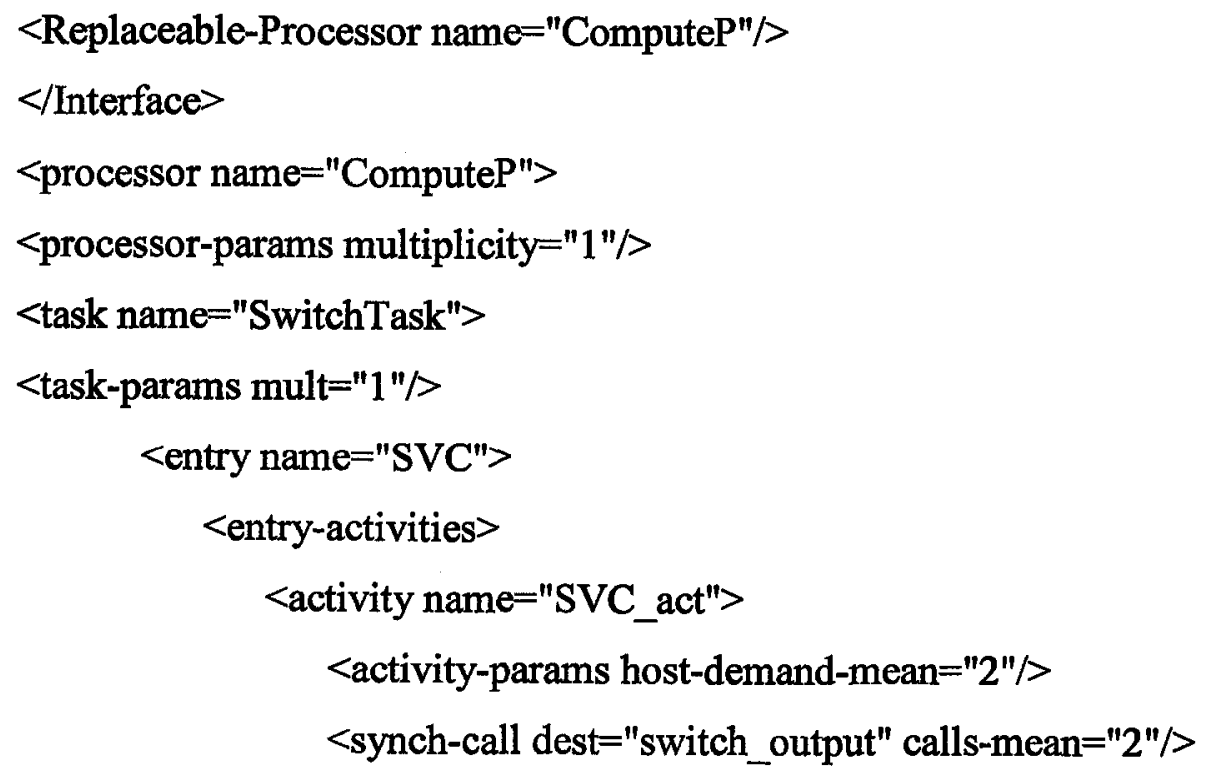




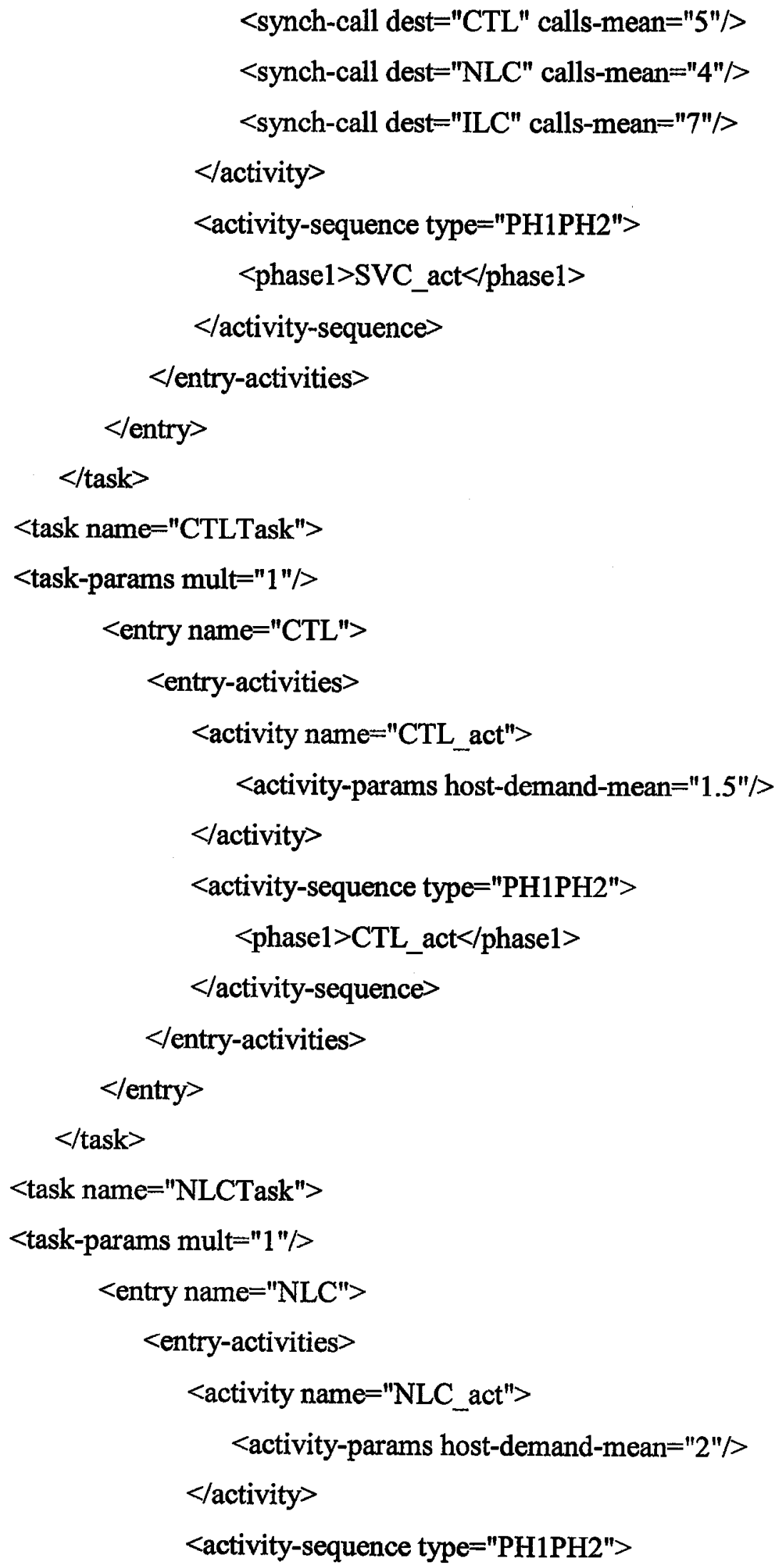




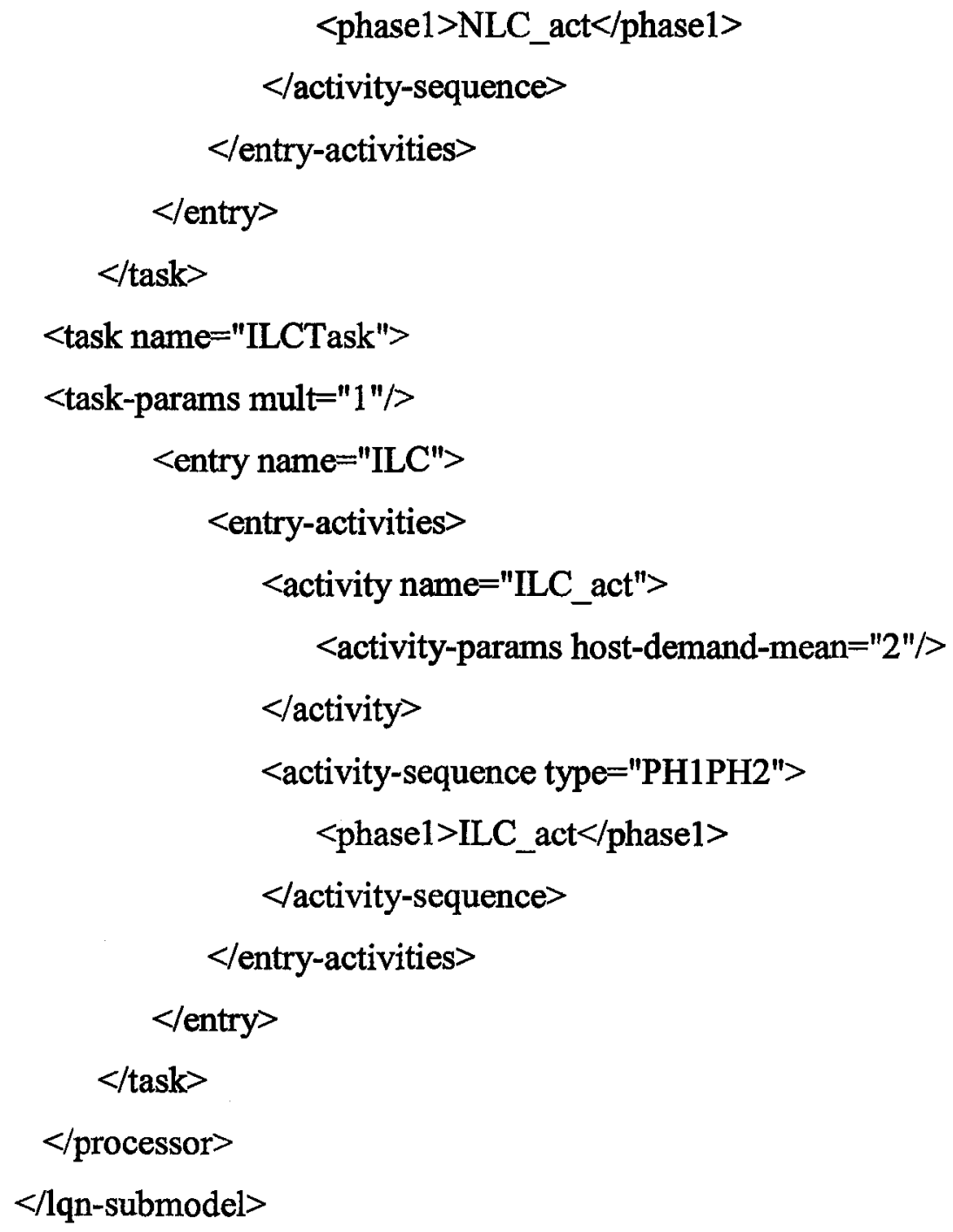

\section{B.10 Component C1 at Figure 5-11}

<lqn-submodel name="CCA1" description="A submodel for Application Server in XML" xmlns:xsi="http://www.w3.org/2001/XMLSchema-instance" xsi:noNamespaceSchemaLocation="lqn-sub.xsd">

$<$ Interface $>$

$<$ in-port name="Input" connect-to="SS7InIp" description="Reporting request controller"/>

<out-port name="TermOut" connect-from="H248Ip" description="big report request"/> 


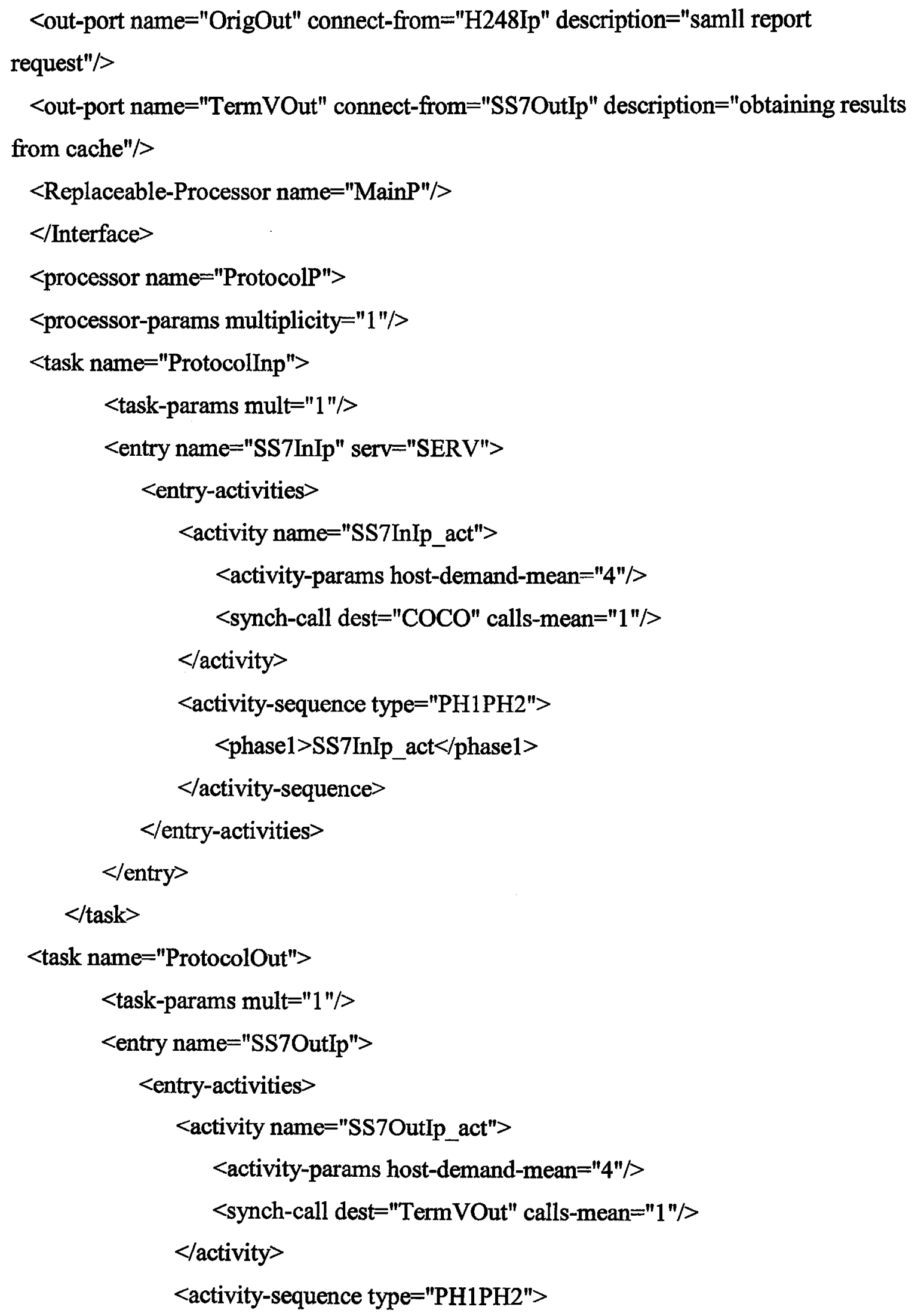




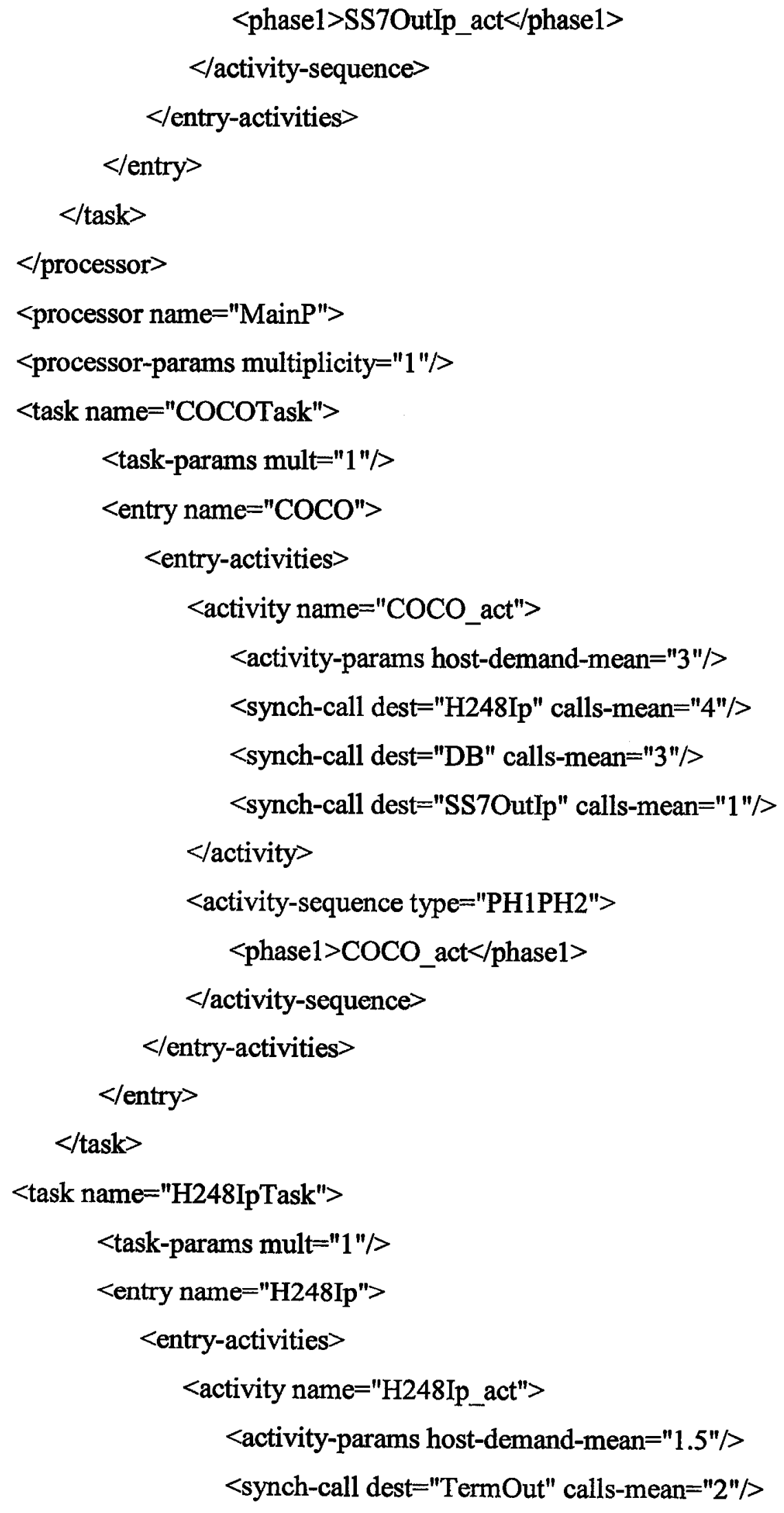




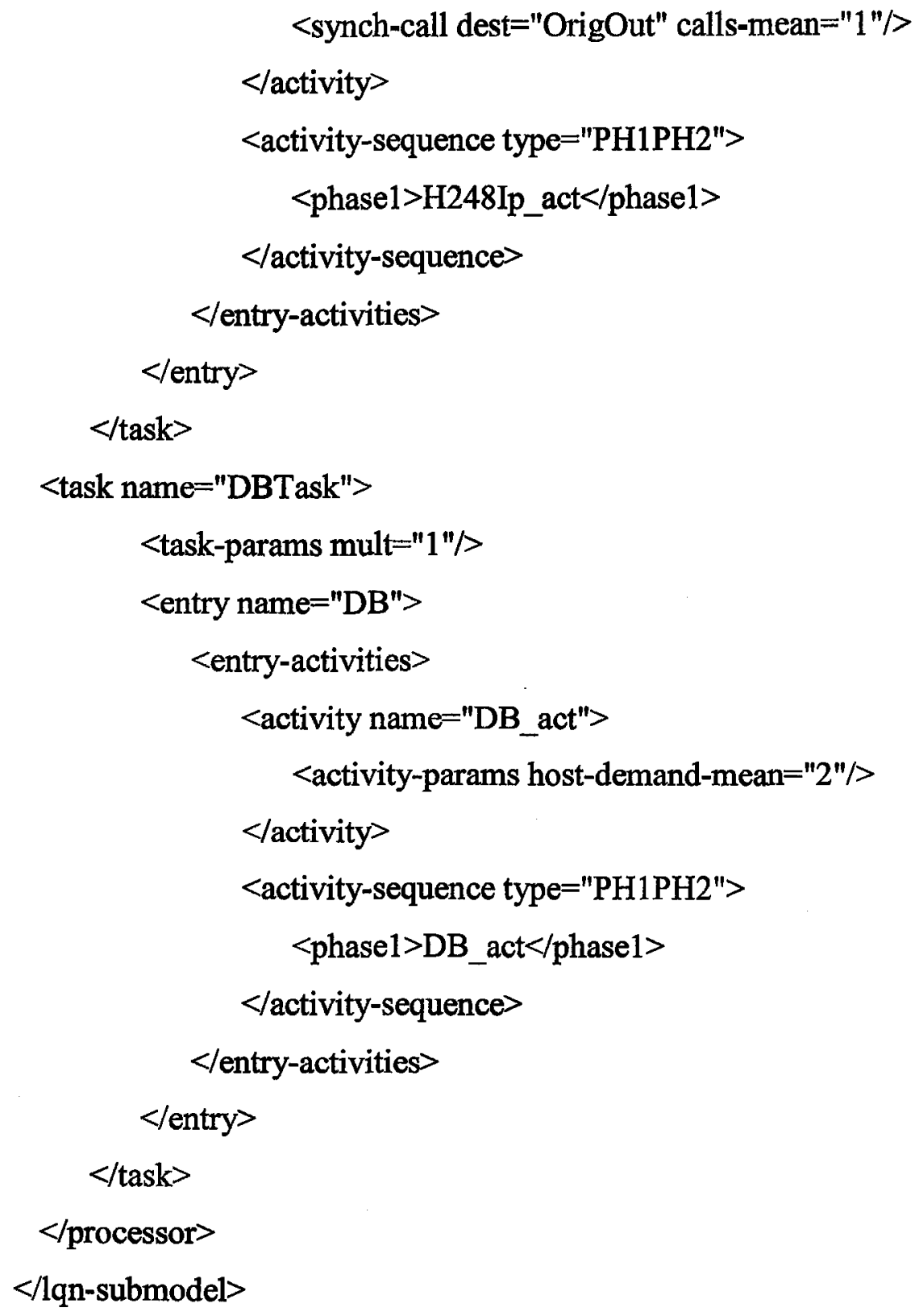

\section{B.11 Component $\mathrm{C} 2$ at Figure 5-12}

$<$ lqn-submodel name="CCA2" description="A submodel for Application Server in XML" xmlns:xsi="http://www.w3.org/2001/XMLSchema-instance" xsi:noNamespaceSchemaLocation="lqn-sub.xsd"> $<$ Interface $>$

$<$ in-port name="Input" connect-to="SS7InIp" description="Reporting request controller"/> 
<out-port name="TermOut" connect-from="H248Ip" description="big report request"/>

<out-port name="OrigOut" connect-from="H248Ip" description="samll report request"/>

$<$ out-port name="TermVOut" connect-from="SS7OutIp" description="obtaining results from cache"/>

$<$ Replaceable-Processor name="MainP" $>>$

$</$ Interface $>$

$<$ processor name $=$ "ProtocolP">

$<$ processor-params multiplicity="1"/>

<task name="ProtocolInp">

$<$ task-params mult="1">

<entry name="SS7InIp" serv="SERV">

<entry-activities>

<activity name="SS7InIp_act">

<activity-params host-demand-mean="4"/>

<synch-call dest="COCO" calls-mean="1"/>

$<$ /activity $>$

$<$ activity-sequence type="PH1PH2">

$<$ phase1 $>$ SS7InIp_act $<$ /phase1 $>$

$</$ activity-sequence $>$

$</$ entry-activities>

$</$ entry $>$

$<$ task $>$

$<$ task name="ProtocolOut">

$<$ task-params mult="1"/>

<entry name="SS7OutIp">

<entry-activities $>$

<activity name="SS7OutIp_act">

<activity-params host-demand-mean="4"/>

<synch-call dest="TermVOut" calls-mean="1"/> 


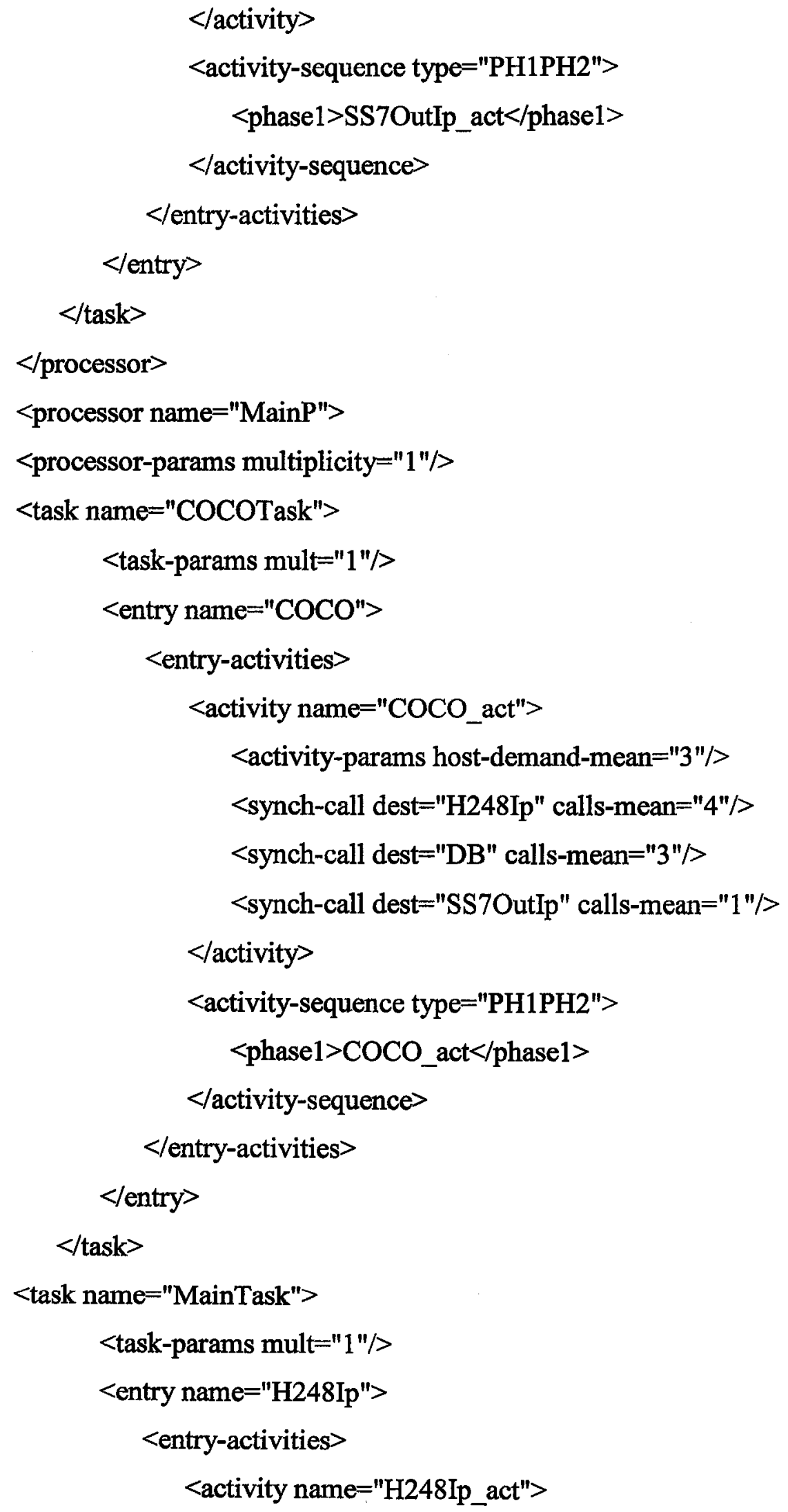




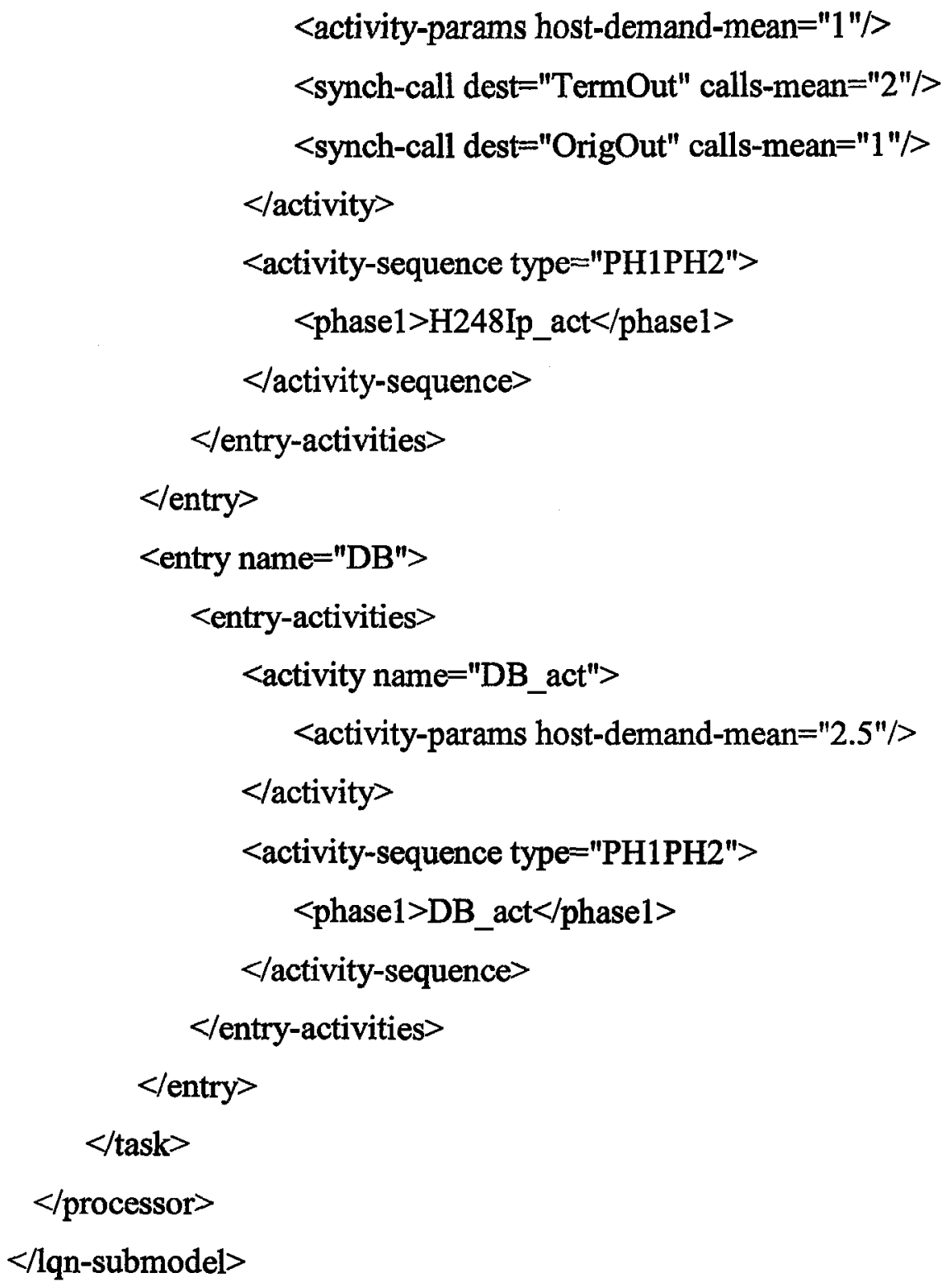

$</$ lqn-submodel $>$ 